\title{
Dynamic Energy Budgets and Bioaccumulation: A Model for Marine Mammals and Marine Mammal Populations
}

\author{
by \\ Tin Klanjšček \\ Dipl. Ing. Physics, University of Zagreb, Croatia (2000) \\ Submitted to the Department of Biology in partial fulfillment of the requirements for \\ the degree of Doctor of Philosophy in Biological Oceanography \\ at the \\ MASSACHUSETTS INSTITUTE OF TECHNOLOGY \\ and \\ WOODS HOLE OCEANOGRAPHIC INSTITUTION
}

June 2006

(C) Tin Klanjšček

The author hereby grants to Massachusetts Institute of Technology and Woods Hole Oceanographic Institution permission to reproduce and to distribute copies of this thesis document in whole or in part.

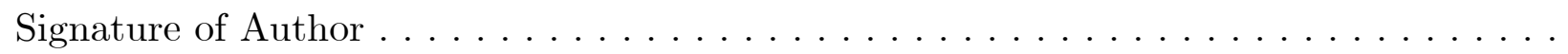
Department of Biology 27 April 2006

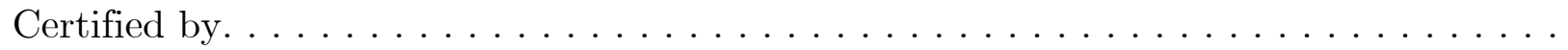
Hal Caswell Senior Scientist, WHOI Thesis Supervisor

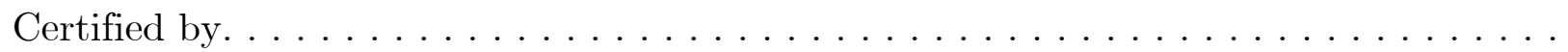

Michael G. Neubert Associate Scientist, WHOI

Thesis Supervisor

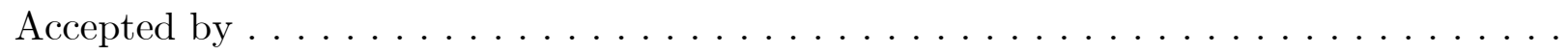

Ed Delong Chairman, MIT/WHOI Joint Committee on Biological Oceanography 


\title{
Dynamic Energy Budgets and Bioaccumulation: A Model for Marine Mammals and Marine Mammal Populations
}

\author{
by \\ Tin Klanjšček \\ Submitted to the Department of Biology \\ in partial fulfillment of the requirements for the degree of \\ Doctor of Philosophy in Biological Oceanography
}

\begin{abstract}
Energy intake of individuals affects growth of organisms and, therefore, populations. Persistent lipophilic toxicants acquired with the energy can bioaccumulate and harm individuals. Marine mammals are particularly vulnerable because of their large energy requirements, and transfer of energy and toxicants from mothers to their young during gestation and lactation. Dynamic energy budget (DEB) models for energy assimilation and utilization, coupled with pharmacokinetic models that calculate distribution of toxicants in individuals, can help investigate the vulnerability.

In this dissertation I develop the first individual DEB model tailored specifically to marine mammals and couple it to a pharmacokinetic model for lipophilic toxicants. I adapt the individual model to the right whale and use it to analyze consequences of energy availability on individual growth, reproduction, bioaccumulation, and transfer of toxicants between generations.

From the coupled model, I create an individual-based model (IBM) of a marine mammal population. I use it to investigate how interactions of food availability, exposure to toxicants, and maternal transfer of toxicants affect populations. I also present a method to create matrix population models from a general DEB model to alleviate some of the drawbacks of the IBM approach.

Thesis Supervisor: Hal Caswell

Title: Senior Scientist, WHOI

Thesis Supervisor: Michael G. Neubert

Title: Associate Scientist, WHOI
\end{abstract}




\section{Acknowledgements}

I will forever be grateful to my advisors, Hal Caswell and Michael G. Neubert, for the freedom I had in choosing my research topic, and their guidance. I am equally grateful to Roger M. Nisbet for dynamically guiding me through the budgets of energetics and hosting me in California. I owe a debt of gratitude to Michael Moore for his ifs and buts on every simplification of marine mammal physiology I suggested, and Mark Hahn and Eric Montie for their help with toxicological aspects of my work. I would also like to thank Daniel Rothman for our discussions that contributed to my understanding of scaling in ecology, and ecology in general.

My friends and the Falmouth community made this alien feel at home. I would like to thank them for their friendship and support; Ivek Ceraj, Vanja Klepi-Ceraja, Beckett Coppola, Michael Desai, Kristin Gribble, Ivona Josip-Ovic, Petra Klepac, Joy Lapseritis, Gareth Outlawson, Enrique Montie, the Muldoon family, Sheri mrs. Michael Simmons, and - last only because the alphabet says so - David Stubinator, thank you. Also, big thanks to my labmates Masami Fujiwara and Christine Hunter for their help and tolerance of the 'creative disorder' on my desk. Jenny, thank you for understanding that a thesis cannot be written in a day.

Julia, Marsha and Laishona, thank you. Please find the strength to change the things you can, the serenity to accept the things you cannot, and the wisdom to know the difference.

Most importantly, I'd like to thank my family — mom, dad, Gaga, baka Kata, baka Jasminka, deda Joza, deda Dali, Vlatka, Karlo and teta Mirka - who have supported me emotionally and financially throughout my student life. The MIT/WHOI joint program was only the last step in their efforts to educate me the best they can despite my objections. I lack the words to describe my gratitude.

This work has been supported by the David and Lucile Packard Foundation, the US National Science foundation (DEB-9973518 and OCE-0083976), the US Environmental Protection Agency (R-82908901-0), the National Oceanographic and Atmospheric Administration (NAO 3NMF4720491) and theWHOI/MIT Joint Program in Oceanography. 


\section{Contents}

1 Introduction $\quad 13$

1.1 Thesis outline . . . . . . . . . . . . . . . . . . . 18

1.2 References . . . . . . . . . . . . . . . . . . . 21

2 Energetics and toxicant bioaccumulation: a model for marine mammals 25

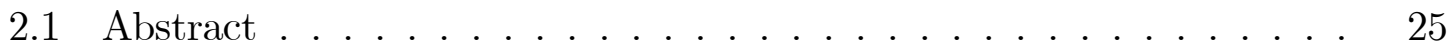

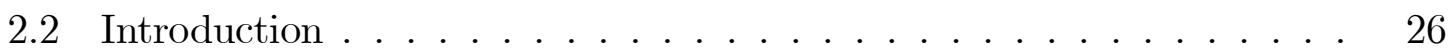

2.3 Model Description . . . . . . . . . . . . . . . . . . . . . . . . 29

2.3 .1 Energetics . . . . . . . . . . . . . . . . . 33

2.3.2 Pharmacokinetics . . . . . . . . . . . . . . . 41

2.4 The Right Whale . . . . . . . . . . . . . . . . . . . . . . 44

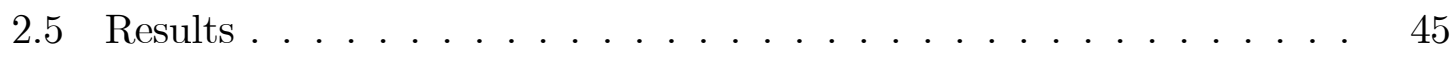

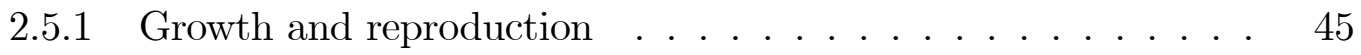

2.5.2 Toxicant distribution and vertical transfer $\ldots \ldots \ldots \ldots$

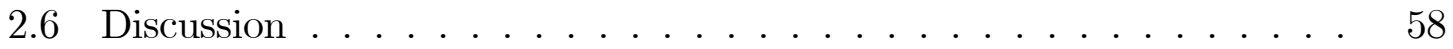

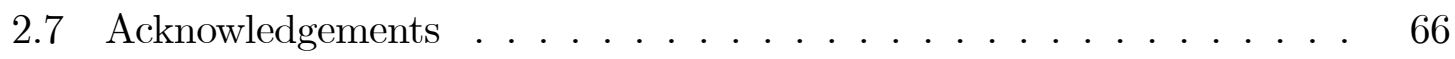

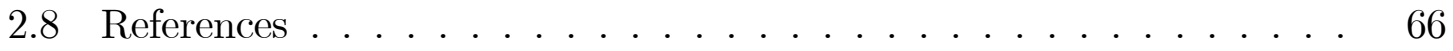

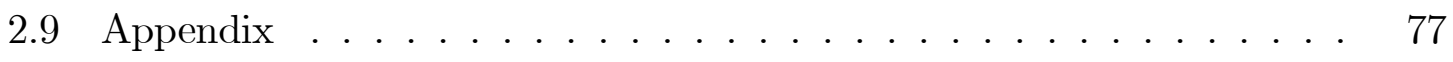

2.9 .1 Morphometrics . . . . . . . . . . . . . . . 77

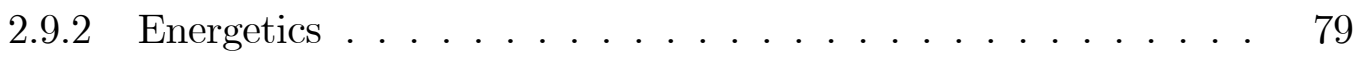


2.9.3 Pharmacokinetics $\left(D_{L S}\right.$ and $\left.C_{I}\right) \ldots \ldots \ldots \ldots$

3 Bioaccumulation and effects of exposure in marine mammal popu$\begin{array}{ll}\text { lations } & 87\end{array}$

3.1 Introduction $\ldots \ldots \ldots \ldots \ldots \ldots \ldots \ldots \ldots \ldots$

3.2 Methods . . . . . . . . . . . . . . . . . . . . 90

3.2.1 Individual energy budget and pharmacokinetic model . . . . . 90

3.2 .2 Modelling mortality . . . . . . . . . . . . . . . . 94

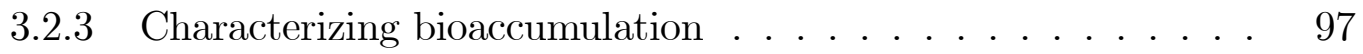

3.2.4 The individual-based model . . . . . . . . . . . . . . . . 98

3.3 Results . . . . . . . . . . . . . . . . . . . . . . . . . . 103

3.3.1 Effects of exposure on bioaccumulation and population growth rate . . . . . . . . . . . . . . . 105

3.3.2 Effects of maternal transfer on bioaccumulation and effects of exposure . . . . . . . . . . . . . . . 107

3.4 Discussion . . . . . . . . . . . . . . . . . . . . . . . . . . . 114

3.5 References . . . . . . . . . . . . . . . . . . . . 118

3.6 Appendix A - values used in figures . . . . . . . . . . . . . . . 122

3.6 .1 Figure $3-5 \ldots \ldots \ldots \ldots \ldots \ldots$. . . . . . . . . . . 122

3.6 .2 Figure $3-6 \ldots \ldots \ldots \ldots \ldots$

3.6 .3 Figure $3-9 \ldots \ldots \ldots \ldots \ldots \ldots$

3.7 Appendix B : Model code . . . . . . . . . . . . . . . . . . 127

4 Integrating Dynamic Energy Budgets into Matrix Population Models $\quad 155$

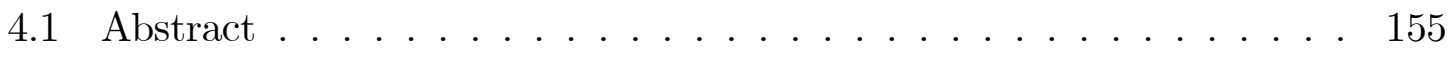

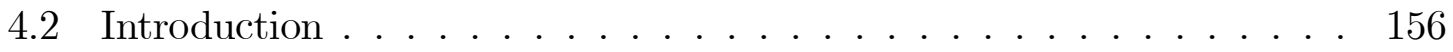

4.2.1 The DEB model . . . . . . . . . . . . . . . . . . . . 157

4.2 .2 The matrix population model . . . . . . . . . . . . 162 
4.3 Methods . . . . . . . . . . . . . . . . . . . . . . . . . . . . 164

4.3 .1 Maturation probability $(\gamma) \ldots \ldots \ldots \ldots \ldots$

4.3.2 Survival probabilities $(\sigma) \ldots \ldots \ldots \ldots \ldots$

4.3 .3 Fecundity $(F) \ldots \ldots \ldots \ldots \ldots \ldots \ldots$

4.3.4 The individual-based model $(\mathrm{IBM}) \ldots \ldots$. . . . . . 167

4.4 Results . . . . . . . . . . . . . . . . . . . . . . . . . . . . 169

4.4.1 Long term (asymptotic) dynamics . . . . . . . . . . . . 169

4.4.2 Seasonal environmental variability . . . . . . . . . . . . 178

4.4.3 Short term (transient) dynamics . . . . . . . . . . . . 180

4.5 Discussion . . . . . . . . . . . . . . . . . . . . . 182

4.6 Acknowledgments . . . . . . . . . . . . . . . . . 185

4.7 References . . . . . . . . . . . . . . . . . . . 185

4.8 Appendix A: Creating a matrix model based on a physiological model 189

4.9 Appendix B - Calculating the stable age distribution . . . . . . . 190

4.10 Appendix C - The extended matrix population model . . . . . . . . 192

4.10 .1 Transitions between size classes, $R_{j l}^{(k)} \ldots \ldots \ldots \ldots$. . . 193

4.10.2 Transitions between energy classes, $Q_{i k}^{(l)}(f) \ldots \ldots \ldots$. . . 195

4.10 .3 Calculating the projection matrix . . . . . . . . . 196

4.10 .4 Preliminary results . . . . . . . . . . . . . . . . . 197

4.10 .5 Seasonal variability . . . . . . . . . . . . . . . . 197

5 Conclusion 205

5.1 References . . . . . . . . . . . . . . . . . . . 212 


\section{List of Tables}

2.1 Compartments and state variables with units. . . . . . . . 30

2.2 Equations for the energy fluxes. . . . . . . . . . . . . 31

2.3 Kinetics: rates of change of state variables. . . . . . . . . . . . 31

2.4 Right whale parameter values. . . . . . . . . . . . . . . . . 32

3.1 Compartments and state variables with units. . . . . . . . 93

3.2 Kinetics: rates of change of state variables. . . . . . . . . . . . 93

3.3 Equations for the energy fluxes. . . . . . . . . . . . . . . . 93

3.4 Right whale parameter values. . . . . . . . . . . . . . . 94

3.5 Figure 5: Growth rate . . . . . . . . . . . . . . . . . 122

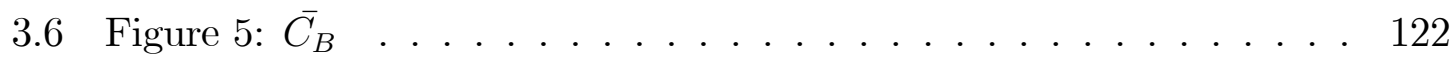

3.7 Figure 5: Reproductive probability . . . . . . . . . . . . 123

3.8 Figure $5: \operatorname{Var}\left(C_{B}\right) \ldots \ldots \ldots \ldots \ldots \ldots \ldots \ldots$

3.9 Figure 6: Influence of maternal transfer on the population growth rate 124

3.10 Figure 6: Influence of the maternal transfer on $\overline{C_{B}} \ldots \ldots \ldots \ldots$

3.11 Figure 6: Influence of maternal transfer on the reproductive probability 125

3.12 Figure 6: Influence of maternal transfer on the variance of $C_{B} \ldots \ldots 125$

3.13 Figure 9: Asymptotic $C_{B} \ldots \ldots \ldots \ldots \ldots \ldots$

3.14 Figure 9: Introduction rate of the toxicant into the population. . . . 126

3.15 Figure 9: Depuration rate of the toxicant from the population. . . . . 126

4.1 Standard parameter values $\ldots \ldots \ldots \ldots$ 
4.2 State variables as a function of age $(s)$ and parameters of the DEB model. For further discussion see Kooijman (2000). . . . . . . . . 160

4.3 Transition counts $n_{i j}$ from stage $j$ to stage $i$. . . . . . . . . . 169

4.4 Comparison of predicted and observed demographic statistics and vital rates. . . . . . . . . . . . . . . . . . . 170

4.5 DEB model equations . . . . . . . . . . . . . . . . . . . . . . . . 191 


\section{List of Figures}

2-1 Marine mammal model outline . . . . . . . . . . . . . . . . 30

2-2 Length of non-reproducing right whales as a function of age for different food availabilities . . . . . . . . . . . . . . . 47

2-3 Influence of reproduction on growth. . . . . . . . . . . . . 48

2-4 Calving interval and age to maturity for a range of food availabilities 50

2-5 Lifetime energy and toxicant distribution for a reproducing female. . 51

2-6 Male and female toxicant bioaccumulation in constant and seasonally

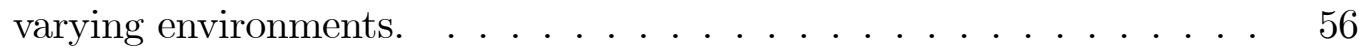

2-7 Toxicant transfer as a function of birth order . . . . . . . . 57

2-8 Effects of biotransformation . . . . . . . . . . . . . . . 59

3-1 Simplified individual model outline . . . . . . . . . . . . . . 92

3-2 Flowchart of the main steps in the simulations. . . . . . . . . . . 101

3-3 Measuring the convergence of the age distribution. . . . . . . . . . . 102

3-4 One simulation of the individual-based model . . . . . . . . . . . . 104

3-5 Effects of exposure on population growth rate and bioaccumulation. . 106

3-6 Effects of maternal transfer on population growth rate and bioaccumulation. . . . . . . . . . . . . . . . . . . . 108

3-7 Bioaccumulation with and without maternal transfer. . . . . . . . 109

3-8 Transient response of bioaccumulation to changes in the toxicant concentration in the environment. . . . . . . . . . . . . . . . . . 112

3-9 Dependance of transients on food availability. . . . . . . . . . . 113 
4-1 The fluxes of energy in a $\kappa$-rule DEB model. . . . . . . . . . . . . . . 158

4-2 Sample solutions of the DEB model . . . . . . . . . . . . . . . 159

4-3 Schematic of the assessment process. . . . . . . . . . . . . 167

4-4 Predicted and observed vital rates and demographic statistics versus ageing acceleration $\left(h_{a}\right) \ldots \ldots \ldots \ldots 171$

4-5 Predicted and observed vital rates and demographic statistics versus

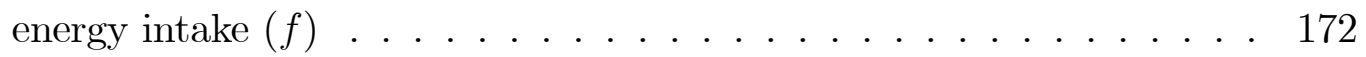

4-6 Predicted and observed vital rates and demographic statistics versus cost of a newborn $\left(C_{N}\right) \ldots \ldots \ldots \ldots$

4-7 Predicted and observed vital rates and demographic statistics versus maximum energy density $\left(\left[E_{m}\right]\right) \ldots \ldots \ldots \ldots$

4-8 Predicted and observed vital rates and demographic statistics versus energy allocation ratio $(\kappa) \ldots \ldots \ldots \ldots$

4-9 Predicted and observed vital rates and demographic statistics versus age to maturity . . . . . . . . . . . . . . .

4-10 Predicted and observed vital rates and demographic statistics versus amplitude of sinusoidal food intake fluctuations $\left(f_{A}\right) \ldots \ldots$. . . . . 179

4-11 Population response to colonization and to a catastrophe . . . . . . . 181

4-12 Two food intake scenarios. . . . . . . . . . . . . . . . . . 199

4-13 Growth rate in a seasonally varying environment. . . . . . . . . . . . 200

4-14 Minimum growth rate as development time increases. See text for discussion. . . . . . . . . . . . . . . . . 201

4-15 An example of a periodic environment. . . . . . . . . . . . . . . 202

4-16 Growth rate as a function of variability and amplitude of environmental fluctuations. . . . . . . . . . . . . . . 203

5-1 Modeling framework . . . . . . . . . . . . 206 


\section{Chapter 1}

\section{Introduction}

It isn't pollution that's harming the environment.

It's the impurities in our air and water that are doing it.

(Unknown)

Marine mammals are awe-inspiring creatures. Many whales traverse the oceans, and blue whales (Balaenoptera musculus) are the largest creatures on the planet. They communicate across tens, possibly hundreds of miles (Dudzinski et al. 2002),

hold their breath for hours, dive to depths of more than 2000m (Stewart 2002), coordinate their behavior, and socialize (Tyack 2002). As many other wild species, they are threatened by human influence: humans compete with them for food and space, hunt them, and pollute their environment. As early as 1972, the US Congress concluded that "certain species and population stocks of marine mammals are, or may be, in danger of extinction or depletion as a result of man's activities", and that "such species and population stocks should not be permitted to diminish beyond the point at which they cease to be a significant functioning element in the ecosystem of which they are a part" (Marine Mammal Protection Act 1972). Understanding marine mammal population dynamics and how it is affected by human interaction is crucial to that goal. 
Since 1925 (Lotka 1925, Volterra 1926), mathematical models have been gaining in popularity as a tool to investigate population dynamics; today, they are widely accepted and utilized. Every mathematical model of a population has to span scales of biological integration by connecting processes of individual growth, reproduction and mortality to population dynamics. The environment and interactions with other species affect these processes and, consequently, population dynamics.

The most prominent environmental factors affecting individual growth, reproduction and mortality include food availability and toxicants. Available food, and the resulting energy intake profoundly affect growth and reproduction. Since toxicants are often bound to the food, the two are - more often than not - intimately connected: energy intake governs toxicant intake, and exposure to toxicants influences individual's mortality, foraging ability and energy utilization (Muller and Nisbet 1997).

To account for these factors, we need to further link scales of biological integration - from biomolecular, to individual, to population-level. Models linking these can be complex and difficult to compute. Pharmacokinetic and energy budget models are widely used to connect biomolecular to individual scales.

Pharmacokinetic models calculate dynamics of a material substance throughout the body of an individual from the exposure of the individual to the substance. The body of the individual may be partitioned into groups of organs and fluids, which then constitute compartments of the pharmacokinetic model. Depending on the compartment, the substance can be assimilated, stored, transformed, and/or excreted on different temporal scales. For example, Hickie et al.(1999) present a pharmacokinetic model for marine mammals in which each of the five compartments operates on a different time scale and blood connects them all.

The results of a pharmacokinetic model include distribution of the modeled substance through compartments and the rate of exchange of the substance between compartments as a function of exposure. This distribution can be translated into effects on the individual using a separate model. If the substance is a toxicant, such 
model is called a toxicant action model.

Pharmacokinetic models usually model one substance. Although an individual is rarely exposed to only one toxicant, tracking one toxicant and its effects on the individual can teach us a great deal about possible patterns of distribution through compartments and effects of other toxicants. This is why pharmacokinetic models are extensively used in medicine to determine proper drug dosage (e.g. Levin et al 1982, Nestorov 2003 (review)) and ecology to determine effects of exposure to toxicants (e.g. Hallam 1989, Moreno et al. 1992, Emond et al. 2005). To track even one toxicant, we need to know the exposure of the individual (toxicant intake). If the toxicant intake and distribution throughout the body depends on energy intake and utilization, we need to understand fluxes of energy within an individual as well.

Energy budget models help calculate the acquisition and utilization of energy for maintenance, growth and reproduction of an individual. They come in a variety of forms; each form constrains the kinds of interaction between the environment and the organism that one can investigate. In general, energy budget models can be classified as either supply- or demand-side models.

In demand-side models individuals always acquire enough energy to satisfy all their energy needs (e.g. von Bertalanffy 1957, Hickie et al. 2000). This is a useful way of estimating toxicant intake for individuals in constant environments, or individuals that are saturated with food. However, most organisms experience variable environments with periods of food scarcity as well as times of surplus, and the growth of many individuals - and populations - is regulated at least in part by food availability. Therefore, demand-side energy budget models cannot yield predictions of population growth as a function of food availability, nor help investigate the interaction of food availability and toxicants.

In supply-side energy budget models, growth and reproduction depend on the available energy. Similar to the pharmacokinetic models, a supply-side model partitions the body of an individual into compartments. These compartments can typically 
be classified as either energy reserve or structure compartments; depending on the purpose of the model, there can be multiple instances of both. Energy reserve compartments act as depositories of energy: an individual can store the energy in them when the energy acquisition is greater than the energy demand, and utilize energy from them when needed. Structure compartments are sinks of energy: the individual either needs to invest energy into them to increase in size, or spend energy to maintain them and stay alive.

Supply-side energy budget models have been pioneered by S.A.L.M. Kooijman in the early 1980s (Kooijman 1986) using Daphnia as the model organism, and developed into a dynamic energy budget (DEB) theory spanning biological scales of integration from biomolecular to population-level, applicable to multiple species. The theory has been developed (Gurney et al. 1990, Hallam et al. 1990, McCauley et al. 1990, Ross and Nisbet 1990) and tested (Noonburg et al. 1998, Nisbet et al. 2000, Nisbet et al. 2004) using mostly Daphnia as the model species, but it can nevertheless explain reproduction of organisms as different as birds and zooplankton using the same principles, and can reproduce the results of demand-side models as special cases of the DEB models (Kooijman 2000). Outputs of DEB models - energy utilization in each of the compartments and the transfer of energy between them - can be readily used as inputs to pharmacokinetic models to investigate the interactions between energy and toxicant intake (e.g. Kooijman and van Haren 1990, Nisbet et al. 1996, Muller and Nisbet 1997).

Despite their versatility, DEB models could not account for the distinctive requirements of marine mammal reproduction and pharmacokinetics because of their fairly rigid form and the way they separate energy for reproduction from energy reserves. In traditional DEB models, energy committed to reproduction is a constant fraction of the flux of energy from the energy reserves. In environments with constant food, this flux depends on the size of the organism only, not the requirements of reproduction. Therefore, the flux from the energy reserves to reproduction cannot change 
during a reproductive event, and the reproductive success depends on the energy set aside for reproduction regardless of the energy available in the reserves. Also, once the reproduction starts, the energy committed to reproduction cannot be utilized for survival by the mother.

Marine mammals, on the other hand, invest energy for reproduction from their energy reserves and often depend on energy intake for successful reproduction. This investment is made only during the reproductive event (pregnancy or nursing), but is substantial, prolonged, and usually significantly depletes the mother's own energy reserves. During reproduction, mother's commitment of energy is a function of the needs of the young. Nevertheless, she can stop the reproduction even during pregnancy (Pitcher at al. 1998), and utilize the unused portion of the energy for survival. Therefore, a marine mammal DEB model should not separate energy for reproduction from mother's energy reserves, and it has to allow a variable energy flux from the reserves to reproduction.

Marine mammals store the bulk of their energy as lipids and transfer large amounts of energy to their young. This puts them at risk from lipophilic toxicants (toxicants that associate preferentially with lipids) such as polychlorinated biphenyls (PCBs). Such toxicants can be hard to biotransform or excrete, and can therefore bioaccumulate and have short term (acute) and long-term (chronic) negative effects on individuals. Maternal transfer of lipids and lipid-bound toxicants between generations increases the potential for negative effects (Aguilar and Borrell 1994, Hickie et al. 2000, Guo et al. 2004). Processes affecting the negative effects of toxicants span multiple temporal scales: from short-term distribution of energy between various tissues affecting acute exposure, to time-scales of individual growth and reproduction affecting bioaccumulation, to multi-generational time scales of maternal transfer affecting exposure.

Traditional pharmacokinetic models successfully separate short-term and individual temporal scales, but traditional DEB models cannot separate temporal scales in 
the manner required by the pharmacokinetic models to describe short-term effects of exposure. The fairly rigid structure of energy flow in traditional DEB models prevents temporal differentiation because it lacks compartments that could account for time scales shorter than energy reserve dynamics. I added such a compartment, and created a new DEB model that can be coupled to pharmacokinetic models spanning the short-term and individual temporal scales.

Marine mammals live long, are relatively few in numbers, and some are in danger of extinction (e.g. Mediterranean monk seal (Panou et al. 1993), North Atlantic right whale (Knowlton et al. 1994), and North Pacific right whale (Brownell et al. 2001)). Therefore, multi-generational experiments on marine mammal are impractical, if not immoral. Population models can help address long-term and population-level questions.

This thesis presents a model for an individual marine mammal, and a model for marine mammal populations. Although tailored to marine mammals, the models are applicable to any other mammalian species that relies primarily on lipids for energy.

I have created a novel dynamic energy budget model and coupled it to a pharmacokinetic model to investigate bioaccumulation of toxicants in marine mammals (Chapter 2), created an individual-based population model to investigate the effects

of this bioaccumulation (Chapter 3), and created a method to link individual energy budgets to a class of readily manageable demographic models with a rich theoretical background, matrix population models (Chapter 4). This was, however, only the start - there are many unanswered questions and many avenues to explore. I outlined some of these questions and started to explore some of the avenues in Chapter 5.

\subsection{Thesis outline}

Chapter $\mathbf{1}$ is the introduction to the thesis. Here I introduce the basic concepts necessary to understand the work presented in the thesis, provide a historical 
background, and provide an overview of the organization of the thesis.

Chapter 2 introduces the marine mammal energy budget model, and demonstrates how to couple it with a pharmacokinetic model to explore bioaccumulation and maternal transfer of toxicants in marine mammals. I apply the model to right whales.

The resulting model is, to the best of our knowledge, the first dynamic energy budget model tailored specifically to marine mammals. Unlike the conventional DEB theory, it does not assume that energy reserves equilibrate with the environment; rather, this equilibration is the result of the inherent dynamics of the model.

Using the model, we addressed the transfer of toxicants from mother to offspring and how food availability affects the transfer. The model opens the way to new general ecophysiological theory, is a potentially powerful tool in marine mammal assessment and management, and offers a source of quantitative predictions that can be tested in the field.

The estimate of the parameters in the Appendix of Chapter 2 is interesting in its own right - parameters are estimated independently whenever possible, sometimes 'borrowed' from other species based on physiological arguments, not fitted so that the outputs of the model match observations, and yet the model predicts the growth and reproduction of right whales very well. We also utilized a number of approaches to estimate the parameters, including careful use of power laws describing interspecific variation in rate processes and a novel representation of the morphology of the right whale. The approach to parameter estimation, as well as the model for the morphology, may be helpful to those wishing to apply the marine mammal energy budget model to another species.

Chapter 3 utilizes the individual model to address population-level questions.

I created an individual based population model (IBM) in which every individual is governed by the individual model from Chapter 2. Using the model, I investigated the 
population growth rate as a function of food availability and exposure to toxicants, and how modes of maternal transfer affect the patterns. I also investigated how the mode of maternal transfer influences the patterns of bioaccumulation in response to sudden changes in environmental toxicant concentration such as may arise from pollution by new toxicants and their subsequent regulation.

Chapter 4 presents and evaluates a method to create a matrix population model from an individual dynamic energy budget model. Whereas an IBM is a good tool to investigate a particular species in a particular food availability and exposure scenario, using it to investigate a large number of food availabilities and exposure scenarios is cumbersome and computationally demanding. Investigating a large number of species, food availabilities and exposure scenarios using the IBM is impractical at best. The matrix population model is much faster to analyze than the IBM because demographic statistics can be calculated directly from the projection matrix.

Although I used a simple, general dynamic energy budget model to develop the method, it is applicable to any energy budget model with the required outputs. Once created, the matrix population model can be analyzed using all the tools of the matrix population theory. Furthermore, investigating effects of additional stressors - such as mortality caused by human interaction - involves commonly practiced and well understood additions to the model.

I differentiated individuals by size only. This proved to be adequate in constant or seasonally varying environments, but a more general model is required to account for annual variability.

In the Appendix $\mathrm{C}$ of the Chapter 4, I suggest a generalization of the methods presented in Chapter 4 to multiple stages and to other state variables of the individual model, such as energy and toxicants.

Chapter $\mathbf{5}$ is the conclusion to the thesis. In it I explore the implications of the thesis and possible uses for the advances made therein. 


\subsection{References}

Aguilar, A., and A. Borrell. 1994. Reproductive transfer and variation of body load of organochlorine pollutants with age in fin whales (Balaenoptera physalus). Archives of Environmental Contamination and Toxicology 27:546 - 554 .

Brownell Jr, R. L., P. J. Clapham, T. Miyashita, and T. Kasuya. 2001. Conservation status of North Pacific right whales. Journal of Cetacean Research and Management, Special Issue 2:269-286.

Congress, U. S., 1972. Marine Mammal Protection Act. Title 16 USC Chapter 31.

Dudzinski, K., J. Thomas, and E. Douaze, 2002. Communication. In Encyclopedia of Marine Mammals. Perrin, W.F., Wursig, B. and Thewissen, J.G.M.(eds.) pp. 248-268. Academic Press.

Emond, C., J. Michalek, L. Birnbaum, and M. DeVito. 2005. Comparison of the use of a physiologically based pharmacokinetic model and a classical pharmacokinetic model for dioxin exposure assessments. Environmental Health Perspectives 113:16661668.

Guo, Y., G. Lambert, C. C. Hsu, and M. M. Hsu. 2004. Yucheng: health effects of prenatal exposure to polychlorinated biphenyls and dibenzofurans. International Archives of Occupational and Environmental Health 77:153-158.

Gurney, W. S. C., E. McCauley, R. M. Nisbet, and W. W. Murdoch. 1990. The physiological ecology of Daphnia : A dynamic model of growth and reproduction. Ecology 71:716-732.

Hallam, T., R. Lassiter, and S. Kooijman. 1989. Effects of toxicants on aquatic populations. Applied Mathematical Ecology 18:352-382.

Hallam, T. G., R. R. Lassiter, J. Li, and L. A. Suarez. 1990. Modelling individuals employing an integrated energy response: Application to Daphnia . Ecology 71:938954.

Hickie, B. E., M. C. S. Kingsley, P. V. Hodson, D. C. G. Muir, P. Beland, and 
D. Mackay. 2000. A modelling-based perspective on the past, present, and future polychlorinated biphenyl contamination of the St. Lawrence beluga whale (Delphinapterus leucas) population. Canadian Journal of Fisheries and Aquatic Sciences $57: 101-112$.

Hickie, B. E., D. Mackay, and J. De Koning. 1999. Lifetime pharmacokinetic model for hydrophobic contaminants in marine mammals. Environmental Toxicology and Chemistry 18:2622-2633.

Knowlton, A. R., S. D. Kraus, and R. D. Kenney. 1994. Reproduction in northatlantic right whales (Eubalaena glacialis). Canadian Journal of Zoology 72:12971305.

Kooijman, S., and R. van Haren. 1990. Animal energy budgets affect the kinetics of xenobiotics. Chemosphere 21:681-693.

Kooijman, S. A. L. M., 1986. Population dynamics on basis of energy budgets. In The dynamics of physiologically structured populations. Metz, J.A.J. and Diekmann, O. (eds). pp. 266-297. Springer-Verlag, Berlin, Germany.

Kooijman, S. A. L. M. 2000. Dynamic energy and mass budgets in biological systems, 2nd edition. Cambridge university press, Cambridge, UK. ISBN 052178608 8.

Levin, S., T. Hahn, H. Rosenberg, and T. Bino. 1982. Treatment of life-threatening viral infections with interferon alpha : pharmacokinetic studies in a clinical trial. Israel journal of medical sciences 18:439-446.

Lotka, A. 1925. Elements of physical biology. Williams and Wilkins Co.

McCauley, E., W. Murdoch, R. Nisbet, and W. Gurney. 1990. The physiological ecology of Daphnia I: Development of a model of growth and reproduction. Ecology 71:703-715.

Moreno, M., K. Cooper, R. Brown, and P. Georgopoulos. 1992. A physiologically based pharmacokinetic model for Mya arenaria. Responses of marine organisms to pollutants. Part 1. Marine Environ Research 34:1-4. 
Muller, E. B., and R. M. Nisbet, 1997. Modeling the effect of toxicants on the parameters of dynamic energy budget models. in ASTM Special Technical Publication. ASTM, Conshohocken, PA, (USA).

Nestorov, I. 2003. Whole body pharmacokinetic models. Clinical Pharmacokinetics 42:883-908.

Nisbet, R., A. Ross, and A. Brooks. 1996. Empirically-based dynamics energy budget models: theory and application to toxicology. Nonlinear Wolrd 3:85-106.

Nisbet, R. M., E. McCauley, W. S. C. Gurney, W. W. Murdoch, and S. N. Wood. 2004. Formulating And Testing A Partially Specified Dynamic Energy Budget Model. Ecology 85:3132-3139.

Nisbet, R. M., E. B. Muller, K. Lika, and S. A. L. M. Kooijman. 2000. From molecules to ecosystems through dynamic energy budget models. Journal of Animal Ecology 69:913 - 926.

Noonburg, E. G., R. M. Nisbet, E. McCauley, W. S. C. Gurney, W. W. Murdoch, and A. M. De Roos. 1998. Experimental testing of dynamic energy budget models. Functional Ecology 12:211-222.

Panou, A., J. Jacobs, and D. Panos. 1993. The endangered Mediterranean monk seal Monachus monachus in the Ionian Sea, Greece. Biological Conservation 64:129140.

Pitcher, K.W., Calkins, D.G. and Pendleton, G.W. 1998. Reproductive performance of female Steller sea lions: an energetics-based reproductive strategy? Canadian Journal of Zoology 76:2075-2083.

Ross, A. H., and R. M. Nisbet. 1990. Dynamic models of growth and reproduction of the mussel Mytilus edulis L. Functional Ecology 4:777-787.

Stewart, B., 2002. Diving behavior. In Encyclopedia of Marine Mammals. Perrin, W.F., Wursig, B. and Thewissen, J.G.M. (eds). pp. 248-268 . Academic Press.

Tyack, P., 2002. Behavior, Overview. In Encyclopedia of Marine Mammals. Perrin, W.F., Wursig, B. and Thewissen, J.G.M. (eds). pp. 87-94 . Academic Press. 
Volterra, V. 1926. Variazioni e fluttuazioni del numero d'individui in specie animali conviventi. Memoria Academia Lincei 2:31-113.

von Bertalanffy, L. 1957. Quantitative laws in metabolism and growth. Quarterly Review of Biology 32:217-231. 


\section{Chapter 2}

\section{Energetics and toxicant}

\section{bioaccumulation: a model for marine mammals}

Work in this chapter has been submitted to Ecological Applications as a manuscript:

Klanjscek, T., R.M. Nisbet, H. Caswell and M.G. Neubert.

Energetics and bioaccumulation: a model for marine mammals.

\subsection{Abstract}

We present a dynamic energy budget (DEB) model for marine mammals, coupled with a pharmacokinetic model of a lipophilic persistent toxicant. Inputs to the model are food availability and lipid-normalized toxicant concentration in the environment. The model predicts individual growth, reproduction, bioaccumulation, and transfer of energy and toxicant from mothers to their young. We estimated all model parameters for the right whale; with these parameters, reduction in food availability increases the age at first parturition, increases intervals between reproductive events, reduces the organisms' ability to buffer seasonal fluctuations, and increases their susceptibility to 
temporal shifts in the seasonal peak of food availability. Reduction in energy intake increases bioaccumulation and the amount of toxicant transferred from mother to each offspring. With high food availability, the toxicant load of offspring decreases with birth order. Contrary to expectations, this ordering may be reversed with lower food availability. Although demonstrated with parameters for the right whale, these relationships between energy intake and energetics and pharmacokinetics of organisms are likely to be more general. Results specific to the right whales include energy assimilation estimates for the North Atlantic and southern right whales, influences of history of energy availability on reproduction, and a relationship between age at first parturition and calving intervals. Our model provides a platform for further analyses of both individual and population responses of marine mammals to pollution, and to changes in food availability, including those likely to arise through climate change.

\subsection{Introduction}

Marine mammals use lipids in their blubber as an energy reserve to mitigate fluctuations in food abundance (Iverson, 2002). They accumulate lipids whenever their energy intake exceeds their expenditures for survival, growth and reproduction. This accumulation can be significant; the blubber typically constitutes a large fraction of a marine mammal's body mass (e.g. up to $43 \%$ in whales (Lockyer 1976) and $50 \%$ in seals (Iverson, 2002)). Energy from the blubber is utilized when energy needs exceed energy inputs (e.g. when starving or reproducing); consequently, the amount of blubber can change significantly from season to season. The rate of change depends upon an individual's energy budget (Reilly, 1991).

To build up large energy reserves, individuals must consume large amounts of food. Because toxicants are often bound-up with food, individuals may ingest large amounts of toxicants as well. Lipophilic and difficult-to-degrade (persistent) toxicants can then accumulate in the blubber, reaching concentrations orders of magnitude greater than 
are found in the food. For example, Ross et al. (2000) measured concentrations as high as $200-300 \mu g$ of total polychlorinated biphenyls (PCBs) per gram lipid in the blubber of killer whales feeding on other species of marine mammals with typical concentrations of $5-50 \mu \mathrm{g} / \mathrm{g}$.

Toxicants may have little effect on the individual while sequestered in the blubber (Joergensen et al. 1999). When an individual uses the energy from the blubber, however, the toxicants can be released and may increase mortality (de Swart et al. 1994, Ross et al. 1996, Martineau et al. 2002) or decrease fertility (Reijnders 1986, Schwacke et al. 2002). These, and other, effects may involve the effects of the mobilized toxicants on an individual's ability to acquire or utilize energy (Muller and Nisbet 1997).

To complicate matters further, toxicants are transferred from mothers to their offspring through milk (Aguilar and Borrell 1994, Restum et al. 1998, Hickie et al. 1999, Ross et al. 2000), exposing these offspring to toxicants during a critical period in their development. The exposure can have adverse impacts, including negative effects on the immune system (Thomas and Hinsdill 1980) and on cognitive abilities (Guo et al. 2004). The amount of toxicant transfer depends on the mother's energetic status and her toxicant burden which, in turn, depend on the environmental conditions she experienced and the consequential energy acquisition and utilization (including reproduction).

Energy and toxicant dynamics are thus intimately and intricately connected. In this paper we report on our efforts to investigate their complex interaction by coupling a mechanistic energy budget model to a pharmacokinetic model for the dynamics of the toxicant.

Energy budget models come in a variety of forms. Any particular form constrains the kinds of interactions between the environment and the organism that one can investigate. In general, energy budget models can be classified as either supplyor demand-side models (Klanjscek et al. 2006). In demand-side models individuals 
acquire enough energy to satisfy all their energy needs (e.g. von Bertalanffy 1957, Hickie et al. 1999). Many previous mammal models (e.g. Porter et al. 2000, 2002 and many references therein) are demand-side models, focusing on adaptations that allow maximum benefit from the consumed food.

However, most organisms experience variable environments with periods of food scarcity as well as times of surplus, and the growth of many populations is regulated, at least in part, by food availability. To handle such variability, one needs a supplyside energy budget model - a model in which growth and reproduction depend on the available energy. Gurney et al. (1990), Hallam et al. (1990), McCauley et al. (1990), Ross and Nisbet (1990), Noonburg et al. (1998), Kooijman (2000), Lika and Nisbet (2000), Nisbet et al. (2000), and Gurney and Nisbet (2004) have all analyzed supply-side models. None of these models, however, take into account the distinctive requirements of mammalian reproduction. Mammals commit energy to reproduction only during reproductive events (gestation and lactation), which require substantial, prolonged and uninterrupted investment of energy. This investment and its success, among other things, depends on the energy intake and energy reserves of the mother.

Here we present a novel energy budget model tailored specifically for marine mammals, and couple it to a pharmacokinetic model (related to Boon et al. 1994). We used the model to investigate the effects of energy availability on bioaccumulation and vertical transfer of toxicants. We tried to make the model as simple as possible; nevertheless, it is complex and has many parameters. Rather than analyzing the model's dynamics for all possible parameter values (a daunting task), we focused on a parameter set that we estimated for the right whale (Eubalaena spp.).

We chose the right whale for several reasons. First, decreased food availability and exposure to persistent lipophilic toxicants have been proposed as factors contributing to the decades-long decline in the North Atlantic right whale population (Knowlton et al. 1994, Fujiwara and Caswell 2001). In the future, we intend to evaluate the significance of these factors relative to others (e.g. ship-strikes and inbreeding). Thus 
our individual-level model is also a first step toward an individual-based populationlevel model that can be used to inform conservation decisions. Second, the life-history of the right whale is not so unusual as to limit our results to them in particular. In fact, we believe that our results are relevant to many marine mammals.

We begin by describing our model in the section Model Description. In the section The Right Whale, we shortly introduce distinctive problems in energetics and toxicology of the right whale. In the Results section, we show how availability and variability of energy affect an individual's growth, age at first parturition, and the average calving interval. We also show how toxicant dynamics depend on the pattern of energy availability. In particular, we show that the vertical transfer of toxicants depends on birth order. Contrary to conventional wisdom (Aguilar and Borrell, 1994, Hickie et al. 2000) and observations (Wells et al. 2005), we show that firstborn individuals do not necessarily receive the largest transfer from their mothers. Finally, we demonstrate that our results are relatively insensitive to the decay rate of the toxicant in the organism. We summarize our most important results and discuss their implications in the Discussion. Details of the model specific to right whales, and step-by-step parameter estimation are in the Appendix.

\subsection{Model Description}

An individual acquires energy needed for its maintenance, growth, and reproduction from the environment. With that energy, the organism acquires toxicants. Both the energy and toxicants are distributed throughout the body. We keep track of these distributions by partitioning the organism into four compartments (Figure 2-1): blood (B), structure (G), structural lipids (S) and lipid energy storage (L). We summarize state variables and their units in 2.1. We summarize the dynamics of the model in Tables 2.2 and 2.3 ; in Table 2.4 we list the parameter values. 


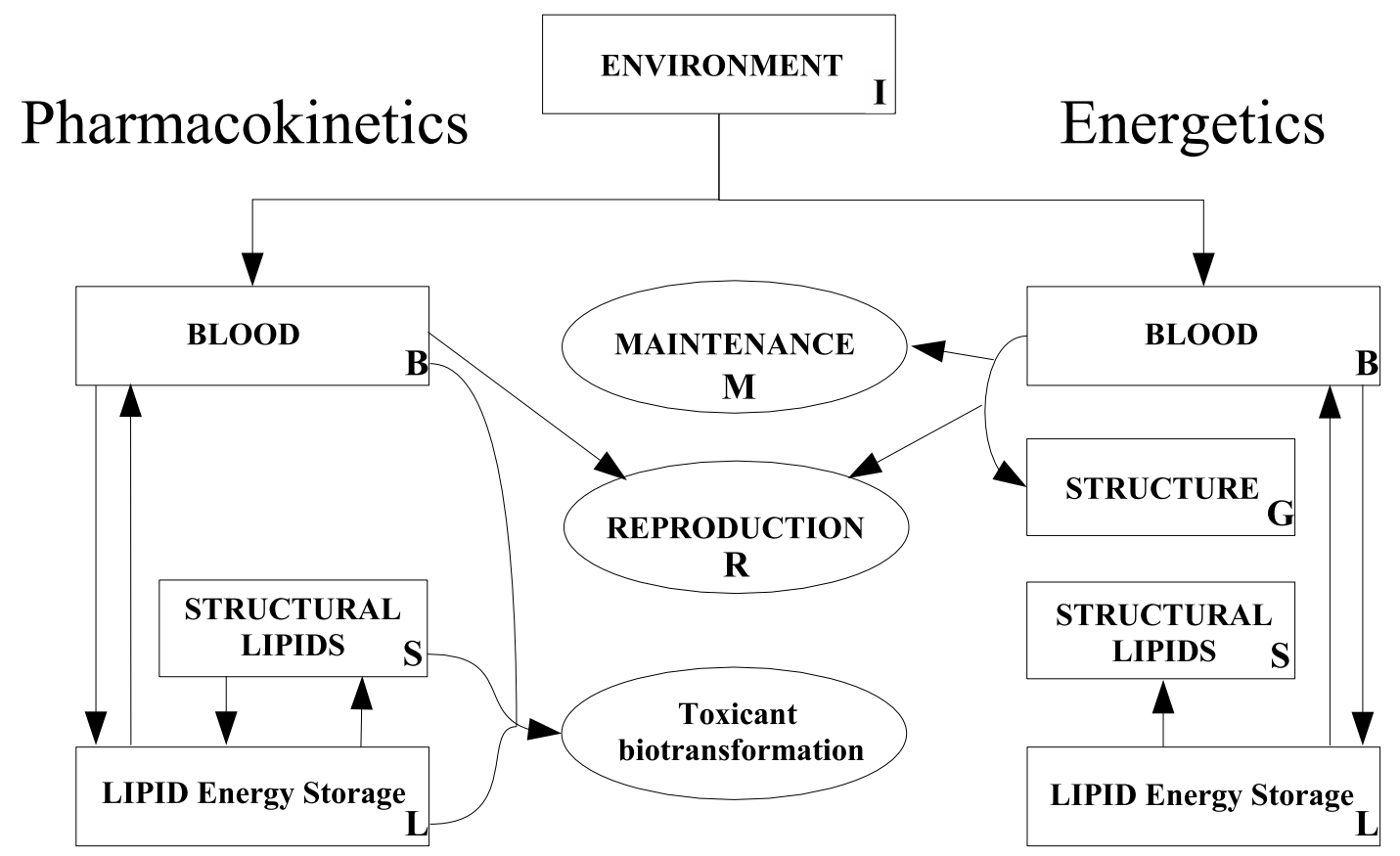

Figure 2-1: Model outline with pharmacokinetic (left) and energetic (right) model compartments. Reproduction (R), metabolism (M) and transformation of toxicants act as sinks for energy, toxicants, or both. Toxicant biotransformation includes all processes that change the molecular form of the modeled toxicant.

Table 2.1: Compartments and state variables with units.

\begin{tabular}{clcc}
\hline \hline & \multicolumn{1}{c}{ Compartment } & Energetics & Toxicology \\
\hline$I$ & Environment & $f$ & $C_{I}[\mathrm{mg} / \mathrm{kg}]$ \\
$G$ & Structure & $V\left[\mathrm{~m}^{3}\right]$ & - \\
$B$ & Blood & $E_{B}[\mathrm{kcal}]$ & $C_{B}[\mathrm{mg} / \mathrm{kg}]$ \\
$L$ & Lipid energy storage & $E_{L}[\mathrm{kcal}]$ & $C_{L}[\mathrm{mg} / \mathrm{kg}]$ \\
$S$ & Structural Lipids & $E_{S}[\mathrm{kcal}]$ & $C_{S}[\mathrm{mg} / \mathrm{kg}]$ \\
$R$ & Reproduction & - & - \\
$M$ & Maintenance & - & - \\
\hline \hline
\end{tabular}


Table 2.2: Equations for the energy fluxes.

\begin{tabular}{ll}
\hline \hline \multicolumn{1}{c}{ Flux [kcal/y] } & \multicolumn{1}{c}{ Description } \\
\hline$F_{I B}=I_{\max } f V^{2 / 3}$ & intake of energy from the environment into blood \\
$F_{B M}=m V$ & energy spent on maintenance \\
$F_{B G}=\left[\beta_{G} E_{B}-F_{B M}\right]_{+}$ & energy utilized for growth ${ }^{1}$ \\
$F_{B L}=\beta_{L} E_{B}$ & energy flux from the blood to the lipid storage \\
$F_{L B}=\beta_{L} k_{L} E_{L}$ & energy flux from the lipid storage to the blood \\
$F_{L S}=e_{S_{0}} \frac{d}{d t} V$ & lipids transformed into structural lipids \\
$F_{B R}=\frac{1}{k_{R}}\left(F_{B R}^{M}+F_{B R}^{G}+F_{B R}^{E}\right)$ & flux of energy to reproduction (details in text) \\
\hline \hline
\end{tabular}

\footnotetext{
${ }^{1}[X]_{+}$is a shorthand notation for $\max (0, X)$.
}

Table 2.3: Kinetics: rates of change of state variables.

\begin{tabular}{cl}
\hline \hline Comp. & \multicolumn{1}{c}{ Dynamics } \\
\hline \multirow{2}{*}{$I$} & $e_{I}=$ function of $t$ \\
& $C_{I}=$ function of $t$ (constant in our simulations) \\
\hline$G$ & $\frac{d}{d t} V=\frac{1}{g} F_{B G}$ \\
\hline \multirow{2}{*}{$B$} & $\frac{d}{d t} E_{B}=F_{I B}+F_{L B}-F_{B L}-F_{B M}-F_{B G}-F_{B R}$ \\
& $\frac{d}{d t} C_{B}=\frac{1}{E_{B}}\left(C_{I} F_{I B}-C_{B}\left(F_{B L}+F_{B R}+\frac{d}{d t} E_{B}\right)+C_{L} F_{L B}\right)-\gamma_{B} C_{B}$ \\
\hline \multirow{2}{*}{$L$} & $\frac{d}{d t} E_{L}=F_{B L}-F_{L B}-F_{L S}$ \\
& $\frac{d}{d t} C_{L}=\frac{1}{E_{L}}\left(C_{B} F_{B L}-C_{L}\left(F_{L B}+F_{L S}+\frac{d}{d t} E_{L}\right)\right)-D_{L S}\left(C_{L}-C_{S}\right) V^{2 / 3}$ \\
\hline \multirow{2}{*}{$S$} & $\frac{d}{d t} E_{S}=F_{L S}$ \\
& $\frac{d}{d t} C_{S}=\frac{1}{E_{S}}\left(C_{L} F_{L S}-C_{S} \frac{d}{d t} E_{S}\right)+D_{L S}\left(C_{L}-C_{S}\right) V^{2 / 3}$ \\
\hline \hline
\end{tabular}


Table 2.4: Right whale parameter values.

\begin{tabular}{|c|c|c|}
\hline Parameter & $\overline{\text { Value }}$ & Description \\
\hline \multicolumn{3}{|c|}{ ENERGETICS } \\
\hline$\beta_{G}$ & $52 \mathrm{y}^{-1}$ & rate of utilization of lipids in blood \\
\hline$\beta_{L}$ & $365 \mathrm{y}^{-1}$ & energy conductivity \\
\hline$m$ & $6.33 \cdot 10^{6} \mathrm{kcal} . \mathrm{m}^{-3} \mathrm{y}^{-1}$ & cost of maintenance for unit of $\mathrm{V}$ \\
\hline$g$ & $4.4 \cdot 10^{6} \mathrm{kcal} / \mathrm{m}^{-3}$ & energetic cost of growing structure \\
\hline$k_{L}$ & 0.02 & equilibrium ratio constant between $\mathrm{B}$ and $\mathrm{L}$ \\
\hline$I_{\max }$ & $2.41 \cdot 10^{7}$ kcal.m ${ }^{-2} \mathrm{y}^{-1}$ & energy acquisition rate per organism's area \\
\hline \multicolumn{3}{|c|}{ PHARMACOKINETICS } \\
\hline$\gamma_{B}$ & $0 \mathrm{y}^{-1}$ & toxicant decay in the blood \\
\hline$D_{L S}$ & $0.09 \mathrm{~m}^{-2} \cdot \mathrm{y}^{-1}$ & toxicant diffusion coeff. between L and $\mathrm{S}$ \\
\hline \multicolumn{3}{|c|}{ REPRODUCTION } \\
\hline$E_{R \text { min }}$ & $1.4 \cdot 10^{8} \mathrm{kcal}$ & minimum stored energy to start reproduction \\
\hline$\tau_{\text {gestation }}$ & $1 \mathrm{y}$ & length of gestation \\
\hline$\tau_{\text {lactation }}$ & $1 \mathrm{y}$ & length of lactation \\
\hline$a$ & $1.25 \mathrm{~m}^{3} / \mathrm{y}$ & rate of growth during gestation \\
\hline$\beta_{v B}$ & $0.35 \mathrm{y}^{-1}$ & von Bertalanffy rate constant \\
\hline$V_{\max }$ & $52.5 \mathrm{~m}^{3}$ & maximum $\mathrm{V}$ under ideal conditions \\
\hline$k_{R}$ & 0.7 & efficiency of reproduction \\
\hline \multicolumn{3}{|c|}{ INITIAL CONDITIONS } \\
\hline$e_{S_{0}}$ & $1500 \mathrm{kcal} / \mathrm{m}^{3}$ & energy density $\left(E_{S} / V\right)$ of the structural lipids \\
\hline$C_{I}$ & $0.035 \mathrm{mg} / \mathrm{kg}$ & lipid-normalized intake toxicant concentration \\
\hline
\end{tabular}




\subsubsection{Energetics}

We assume that all tissue may be characterized as either "energy reserves" or "structure" (Kooijman 2000). The energy reserves are materials that can be utilized as an energy source for maintenance and growth (e.g. non-structural lipids, carbohydrates, and proteins). Any tissue the animal cannot utilize for energy during starvation (e.g. bones, structural lipids etc.) composes the structure. The exact composition of the energy reserves and the structure depend on the species. Some tissues, such as muscle, belong to both energy and structure to some degree: an organism uses muscle protein as energy when starving, but retains some even when it faces death from hunger.

We propose that the energy dynamics of a marine mammal can be captured by focusing on lipid dynamics, as long as the relative amounts of different compounds composing the energy reserves have a constant ratio. For example, muscle protein is depleted in a constant proportion to energy reserves in the blubber during starvation (Struntz et al. 2004 pp 18, Nordoy and Blix 1985). Hence, the dynamics of any component of reserves contains information about other types. We have chosen to keep track of lipids because they are the largest energy reserve in marine mammals, and because lipid dynamics determine the pharmacokinetics of lipophilic toxicants. The proportionality assumption does not hold for some types of energy reserves, e.g. protein and glycogen. This, however, does not influence overall energy dynamics because such types comprise only a small fraction of standing energy reserves; for example, during starvation $94 \%$ of energy consumption in grey seals (Halichoerus grypus) comes from subcutaneous blubber (Nordoy and Blix 1985).

Lipids, and the tissues that hold them, have multiple functions (Struntz et al. 2004, Koopman et al. 2002). The largest pool of lipids is the blubber, but not all lipids in the blubber are readily metabolized. Lipids in the superficial blubber, i.e. lipids in and beneath the epidermal layer are barely metabolically active and can be neglected as a source of energy for the organism (Struntz et al. 2004). The metabolic activity of the blubber increases with depth, and deepest layers are most 
metabolically active (Koopman et al. 2002, Aguilar and Borrell 1990). Recognizing this, we lump all metabolically inert lipids, such as those in the superficial blubber, into the "structural lipids" compartment (S), and all metabolically active lipids, such as those in the middle and deep layers of the blubber, into the "lipid energy storage" compartment (L).

The structure compartment $(G)$ includes all the structure except the structural lipids, and we assume that its composition remains constant through ontogeny. We further assume isomorphic growth, with the implication that the structural volume $(V)$ of the animal is proportional to the cube of some measure of its length. We use $V$ as the state variable representing structure. The blood (B) mediates all transformations of energy and toxicants on short time scales, such as those in the gut and in the liver.

The dynamics of the energetics model is determined by fluxes (rates of flow of energy) between compartments. We denote a flux from compartment $X$ into compartment $Y$ with $F_{X Y}$.

\section{Growth $\left(F_{B G}\right)$ and maintenance $\left(F_{B M}\right)$ of structure $\mathbf{G}$}

We assume the energy flux to growth and maintenance is proportional to the lipids available in the blood $\left(E_{B}\right)$, with a constant of proportionality that characterizes the rate of utilization of lipids, $\beta_{G}$. Maintenance has priority; an organism can utilize energy for growth only after it meets the energy requirement for maintenance.

The energy costs of maintenance depend on the size of the organism, and its energy expenditures for foraging and migration. We follow the dynamic energy budget (DEB) theory of Kooijman (2000) and assume that these costs are proportional to the volume of the organism. Hence, the energy flux $F_{B M}$ required for maintenance of an organism of volume $V$ is

$$
F_{B M}=m V,
$$

where $m$ is the energy required per unit of time to maintain a unit of volume. 
The flux of energy to growth, $F_{B G}$, is the flux possible after maintenance has been met:

$$
F_{B G}=\left[\beta_{G} E_{B}-F_{B M}\right]_{+},
$$

where $[x]_{+}$is a short-hand notation for $\max (x, 0)$. If the energetic cost of growth by a unit of volume is $g$, the rate of growth of the organism is:

$$
\frac{d}{d t} V=\frac{F_{B G}}{g} .
$$

\section{Energy assimilation $\left(F_{I B}\right)$}

Only a fraction of the energy intake is assimilated and transported by the blood throughout the body. Hence, the flux of energy from the environment to the blood $\left(F_{I B}\right)$ depends on food density in the environment, the organism's foraging ability, its ability to process food, and its energy assimilation efficiency. We assume isomorphic growth, so that the energy intake from the environment is proportional to the area of the feeding structures (e.g. surface of the baleen), which is proportional to the surface area of the organism. Then,

$$
F_{I B}=I_{\max } f V^{2 / 3},
$$

where $I_{\max }$ is the maximum assimilation rate per unit area, and $f$ a saturating, Type II function of $e_{I}$, the environmental energy density:

$$
f=\frac{e_{I}}{k_{I}+e_{I}},
$$

where $k_{I}$ is the half-saturation constant. Throughout the paper, we refer to $f$ as energy availability. Since every organism has different food types and foraging patterns, the exact meaning of these parameters needs to be determined separately for each organism (see Gurney and Nisbet 1998, pp. 87 for details). 
The energy intake determines the ultimate size of the organism, $V_{\infty}$, and the maximum size of the organism, $V_{\max }$. At $V_{\infty}$, in a hypothetical constant environment and when not diverting energy into reproduction, the organism spends all the acquired energy on maintenance, i.e. $F_{I B}=F_{B M}$. From (2.1) and (2.4),

$$
V_{\infty}=\left(\frac{I_{\max } f}{m}\right)^{3}
$$

The maximum size is attained for $f=1$ :

$$
V_{\max }=\left(\frac{I_{\max }}{m}\right)^{3}
$$

\section{Dynamic equilibrium between blood and lipid energy storage $\left(F_{B L}\right.$ and $\left.F_{L B}\right)$}

The blood and lipid energy reserves are in direct contact and, therefore, try to equilibrate through exchange of lipids. We assume the flux from one compartment into another depends linearly on the amount of lipids in the origin compartment, and does not depend on anything in the destination compartment. Then, the flux of lipids from $\mathrm{B}$ to $\mathrm{L}\left(F_{B L}\right)$ and $\mathrm{L}$ to $\mathrm{B}\left(F_{L B}\right)$ are:

$$
\begin{aligned}
& F_{B L}=\beta_{L} E_{B} \text { and } \\
& F_{L B}=\beta_{L} k_{L} E_{L} .
\end{aligned}
$$

The net transport of lipids is equal to the difference between the two fluxes.

\section{Growth of structural lipids $\mathbf{S}\left(F_{L S}\right)$}

Growth is the only process relevant to structural lipids, and it is proportional to the growth of structure:

$$
\frac{d}{d t} E_{S}=\frac{E_{S}}{V} \frac{d}{d t} V
$$


The biggest pool of structural lipids - the external blubber stratum - is not metabolically active, and does not differ significantly in composition between demographic groups (Aguilar and Borrell 1990). This holds for acoustic fats as well. Structural lipids are typically not significantly vascularized and are, therefore, not metabolically active. This leads us to assume that structural lipids are made from energy storage lipids directly by gradual processes such as de-vascularization, rather than through blood. Hence, the only flux to the compartment $\mathrm{S}$ is the flux from L:

$$
F_{L S}=e_{S_{0}} \frac{d}{d t} V
$$

where $e_{S_{0}}$ is the proportion of lipids in the structure of the organism.

\section{Reproduction $\left(F_{B R}\right)$}

After conception, mammalian reproduction has two parts: gestation and lactation. We model them separately because they have different modes of energy and toxicant transfers. In gestation, the mother transfers energy and toxicants through the placenta. During lactation, the mother transfers energy and toxicants through milk.

We assume that females conceive if, during the reproductive season, the energy in their lipid energy storage is greater than a certain critical value, $E_{R}$. This assumption is consistent with the observed low variation of lipid storage energy density in female fin whales (Aguilar and Borrell 1990), suggesting that they reproduce upon reaching a certain 'trigger' lipid storage energy density. Whether female fin whales accumulate that energy after becoming pregnant, or become pregnant because they have reached the energy density is not clear. Nevertheless, given that onset of ovulation in some mammals depends on their energy reserves (Frisch et al. 1975, Van der Spuy 1985, Frisch 1990, but see Bronson and Manning 1991), that reproductive performance in mammals which experience seasonal food fluctuations depends on energy reserves of mature females (Frisch 1978, Gopalan and Naidu 1972, Lee 1987), and that fin 
whale fecundity seems to be food-limited (Lockyer 1986), it is plausible to assume that marine mammals trigger ovulation depending on available energy storage. This view is corroborated for right whales by observations (Angell 2005). We assume that there are always enough males present that, upon ovulation, a female is fertilized and becomes pregnant.

The flux of energy to reproduction includes the flux needed for maintenance $\left(F_{B R}^{M}\right)$, growth $\left(F_{B R}^{G}\right)$, and increase of energy reserves $\left(F_{B R}^{E}\right)$ of the young mammal during gestation and lactation:

$$
F_{B R}=\frac{1}{k_{R}}\left(F_{B R}^{G}+F_{B R}^{M}+F_{B R}^{E}\right)
$$

where $k_{R}$ is the reproductive efficiency of utilization of energy, potentially different between gestation and lactation.

We assume that mother is able to meet all energetic needs of the calf during gestation. We use an empirical model for fetal development commonly used for mammals (Martin and MacLarnon 1985), combined with the assumption that the mass of the fetus is proportional to its volume. According to the model, the volume of the fetus, $V_{F}$, at time $\tau \geq 0.2 \tau_{\text {gestation }}$ since conception is

$$
V_{F}(\tau)=a\left(\tau-0.2 \tau_{\text {gestation }}\right)^{3}
$$

The volume of the fetus and the rate of change of the volume determine the energy needs of the fetus and, therefore, the mother's energy flux to reproduction.

Total energy flux to reproduction during gestation for $\tau \geq 0.2 \tau_{\text {gestation }}$ includes the flux for maintenance of the fetus,

$$
F_{B R}^{M}(\tau)=m V_{F}(\tau)
$$


growth of the fetus,

$$
F_{B R}^{G}(\tau)=g \frac{d}{d \tau} V_{F}(\tau)
$$

and energy transferred to the fetus to build its energy reserves. In our model, the fetus acquires lipid energy reserves throughout gestation even though during fetal development energy is directed mainly towards growth, and lipid energy reserves are developed in the late stages of fetal development (Struntz et al. 2004). Energetically, the timing is not an issue because there is no cost associated with storing reserves, and only total amounts of matter transferred. For the same reason, the timing does not affect estimates of toxicant transfer because the toxicant transfer mainly depends on the total amount of lipids transferred. It may not be a significant issue for estimating gestational exposure either, because the fetus does not experience major bioaccumulation during gestation (the concentration of toxicants in its blood equilibrates with the mother's).

When connecting the energetics of gestation to pharmacokinetics, we assume that there is no placental barrier to toxicant transfer, and therefore the calf's and the mother's concentration of the toxicant in the blood tend to equilibrate. The validity of this assumption is not vital to our model because the bulk of energy (and, therefore, toxicant) is transferred during lactation (Young 1976). However, if exposure during fetal development is of concern, a more detailed model of fetal development, including the transport of lipids and toxicants across the placenta, may be required.

We assume that energy in the blood of the fetus is just sufficient to provide the energy flux for maintenance, and that the energy in the lipid energy storage compartment is in a dynamic equilibrium with the lipids in the blood:

$$
\begin{aligned}
E_{B}^{\text {Fetus }} & =\frac{1}{\beta_{L}} F_{B R}^{M}, \\
E_{L}^{\text {Fetus }} & =\frac{1}{\beta_{L} k_{L}} F_{B R}^{M} .
\end{aligned}
$$

The energy flux from the mother required to satisfy (2.16-2.17) and the increase in 
the structural blubber, for $\tau \geq 0.2 \tau_{\text {gestation }}$, is the energy needed to increase energy pools of the fetus proportionally to the change in volume:

$$
F_{B R}^{E}=\left(\frac{1}{\beta_{L}}\left(1+\frac{1}{k_{L}}\right) m+e_{S 0}\right) \frac{d}{d \tau} V_{F}(\tau)
$$

After birth, a newborn depends exclusively on its mother's milk for energy until weaning (Thomas and Taber 1984). During nursing, there are two competing processes: what the nursling demands and what the mother can give. The energy transferred is equal to the lesser of the two after adjusting for the inefficiencies of milk production and nursing. We assume that the nursling has an "ideal energy demand" which would allow it to grow following the von Bertalanffy growth curve, $V_{v B}(t)$, with its ultimate goal to reach the maximum volume observed for the species $\left(V_{\max }\right)$. The energy flux required to meet the target growth curve $V_{v B}(t)$ is the sum of energy fluxes needed for maintenance, growth and increasing energy reserves of the nursling:

$$
\begin{aligned}
& F_{B R}^{M}=m V_{v B}(t), \\
& F_{B R}^{G}=g \frac{d}{d t} V_{v B}(t), \text { and } \\
& F_{B R}^{E}=\left(e_{B_{0}}+e_{L_{0}}+e_{S_{0}}\right) \frac{d}{d t} V_{v B}(t) .
\end{aligned}
$$

Here we assume that the nursling tries to match the energy density of its mother at conception, $e_{B_{0}}$ in the blood, and $e_{L_{0}}$ in the lipid storage compartment.

Using our model, we calculate the growth of the nursling from its actual energy assimilation, which is the minimum between the ideal energy demand and what the mother can provide. When the mother is not able to meet the ideal energy demand, the nursling receives less then ideal energy flux. If this flux combined with the nursling's energy reserves is not sufficient to meet the maintenance requirements of the nursling, the nursling dies. 


\subsubsection{Pharmacokinetics}

Our pharmacokinetic model keeps track of lipid-normalized concentrations of toxicants in an individual (Table 2.1) by modeling the biotransformation and movement of lipophilic toxicants between compartments of the organism. Unless otherwise mentioned, all concentrations are lipid-normalized, expressed in milligrams of toxicant per kilogram of lipid $(\mathrm{mg} / \mathrm{kg})$. Upon entering the blood, the toxicants can either be biotransformed (e.g. hydroxylated (Borga et al. 2004)), or transported throughout the body.

With the exception of the compartment G (structure without structural lipids), compartments in the pharmacokinetic model correspond to those of the energetics model. The compartment $\mathrm{G}$ is not directly involved in the toxicant dynamics because it does not include any lipids.

Lipophilic toxicants are not completely free to diffuse between compartments, nor are they all covalently bound to the lipids. Therefore, the transport of toxicants between compartments is a mixture of passive transport where toxicants behave as if they were not bound at all to the lipids, and lipid-facilitated transport where toxicants behave as if they were covalently bound to the lipids. We model both modes of transport.

Facilitated transport is assumed to be completely controlled by the fluxes of energy in the energetics model: the toxicant flux from one compartment to another is proportional to the concentration of the toxicant in the source compartment and the flux of lipids from the source to the destination compartment. We assume no barriers to facilitated toxicant transport between compartments.

Passive transport involves the diffusion of toxicants between compartments. Diffusion rate is proportional to the difference in concentrations of toxicants, and to the boundary area between the compartments (Crank 2004) which, in view of our assumptions of an isomorphic animal, is assumed proportional to $V^{2 / 3}$. Therefore, the rate of change of concentration of toxicants in compartments $X$ and $Y$ due to 
diffusion is:

$$
\frac{d}{d t} C_{Y}=-\frac{d}{d t} C_{X}=D_{X Y}\left(C_{X}-C_{Y}\right) V^{2 / 3}
$$

Regardless of the method of transport, we assume the toxicants redistribute within compartments instantaneously, i.e. the concentration within any compartment is uniform.

Although the model can account for biotransformation of toxicants in all compartments (Figure 2-1), the rates of biotransformation in the blood compartment are higher than in other compartments (Boon 1992, Borga et al. 2004). Furthermore, the other compartments communicate with the blood on time-scales much shorter than rates of biotransformation in those compartments. Therefore, we can simplify the model by assuming that only the biotransformations of the toxicants in the blood (e.g. by liver, gut and vascular endothelia) are significant. We represent these biotransformations as a sink of toxicants - when biotransformed, toxicants are lost from the model.

Aside from the dilution by growth (proportional to $-C_{X} \frac{d}{d t} E_{X}$ for any compartment $X)$, the rate of change of toxicant concentration of any compartment is determined by its sources, sinks, and passive and/or facilitated exchange of toxicants with other compartments. We do not model feedback of contaminants on rate processes (e.g. Leung et al. 1990a, Leung et al. 1990b), but such feedback could be incorporated if necessary. The environment is the original source of all the accumulated toxicants.

Because of our choices of units motivated by the literature, we need a conversion factor $\eta$ to connect fluxes of energy $([\mathrm{kcal} / \mathrm{y}])$ to fluxes of lipids $([\mathrm{kg} / \mathrm{y}])$. The factor has units of $\mathrm{kg}$ lipid per $\mathrm{kcal}(\mathrm{kg} / \mathrm{kcal})$. We do not need to know its value, as it cancels out in the equations for rates of change of toxicant concentrations (Table 2.3). 


\section{Blood compartment (B)}

We assume that toxicants in the blood experience both facilitated and passive transport to and from lipid energy storage. Fluxes of lipids to and from the blood compartment are both large, even when the standing stock $\left(E_{B}, C_{B}\right)$ is small. Because of this, we assume that the dominant mode of transport of toxicants between the blood and the lipids is facilitated and ignore passive toxicant transport in and out of the blood compartment. Facilitated transports include the environmental input $\left(\eta C_{I} F_{I B}\right)$, the exchange with the lipid energy storage $\left(\eta\left(C_{L} F_{L B}-C_{B} F_{B L}\right)\right)$ and a sink: reproduction $\left(-\eta C_{B} F_{B R}\right)$.

Additional sinks include biotransformation $\left(-\gamma_{B} C_{B}\right)$, urinary excretion, and respiratory exchange. Urine is not rich in lipids and, according to our assumptions, cannot be a large sink for non-metabolized lipophilic toxicants. Breathing is potentially both a source and a sink; we assume, however, that the respiratory exchange of lipophilic toxicants is much smaller than the nutritional input and can, therefore, be ignored. Hence, we ignore urinary excretion and respiratory exchange because we deem them not important, cannot parameterize them reliably, and account for them (at least partially) through biotransformation. These processes can be included in the model at a later date if necessary. Note that fecal excretion is accounted for by the assimilation efficiency (which is equal to the assimilation efficiency of energy): some lipids pass through the digestive system, and so do the toxicants associated with them.

\section{Lipid energy storage (L)}

Facilitated transport includes transfers between the lipid energy storage and the blood $\left(\eta\left(C_{B} F_{B L}-C_{L} F_{L B}\right)\right)$ and a sink from the toxicant flux associated with the growth of the structural lipids $\left(-\eta C_{L} F_{L S}\right)$. Passive transport consists of the diffusion between the two types of lipids $\left(-D_{L S}\left(C_{L}-C_{S}\right) V^{2 / 3}\right)$. 


\section{Structural lipids (S)}

Since we assume that the structural lipids are created from the energy storage lipids, their exchanges of toxicants include only the flux from the energy storage lipids during creation of structural lipids $\left(\eta C_{L} F_{L S}\right)$ and diffusion with the energy storage lipids $\left(D_{L S}\left(C_{L}-C_{S}\right) V^{2 / 3}\right)$. Additional losses of toxicants could include losses through shedding of skin. We did not find evidence that shedding comprises a big sink, and thus ignored it.

\subsection{The Right Whale}

There are three species of right whales: the North Pacific (Eubalaena japonica), the North Atlantic (Eubalaena glacialis) and the Southern (Eubalaena australis) right whale (Rosenbaum et al. 2000). There are possibly additional stocks within these populations (The North Atlantic right whale recovery team 2000). Prior to the ban on right whale hunting in 1935 (Convention, 1931), all right whales had been commercially exploited and brought to dangerously low levels. The Southern right whale recovered since the ban and exhibits a yearly population growth rate of more than 7\% (Best et al. 2001). The recovery of the North Pacific right whales seems to be threatened by illegal hunting, but more research is needed to quantify their status (Brownell et al. 2001). The North Atlantic right whale was hunted down from as many as 1900 whales in 1630 to as few as 50 in the 1800s (Reeves et al. 1992). Since the ban on hunting, it has recovered to the estimated 300 individuals today (Kraus et al. 2001). In spite of this small recovery, the Northern Atlantic right whale seems to be declining again with an increasing rate. If these trends persist, the North Atlantic right whale is expected to go extinct in about 200 years (Fujiwara and Caswell 2001). Some insight into demographic reasons for the continuing decline can be gained by comparing the North Atlantic whales with their southern cousins: the North Atlantic right whale has twice the mortality rate, while their calving interval is almost double 
that of the southern right whale (Kraus et al. 2001, Best et al. 2001, Brunell 2001).

Whereas gear entanglement and ship strikes account for most of the higher mor-

tality (Fujiwara and Caswell 2001), and reducing these causes may be necessary for their recovery, it is also important to understand why their calving interval is so long. The reason may be the fact that right whales need large amounts of energy for growth, maintenance and reproduction, which may not be available in the environment. They also may be at risk from toxicants because, even though right whales are not high in the food chain because they feed mainly on zooplankton, their lipidrich nature and marine mammal life history makes them potentially vulnerable to persistent bioaccumulating compounds such as PCBs. Therefore, a combination of nutritional stress and exposure to toxicants may be increasing the interval between successful reproductions and reducing the fertility (Knowlton et al. 1994, Angell et al. 2005).

Right whales can also experience additional hazards due to starvation-induced exposure when inactive toxicants stored within the lipids get mobilized as the lipids get utilized (Aguilar et al. 1999). This is of a particular concern because right whales fast during a part of the year (Best and Schell 1996) and nutritional stress could interact with such exposure to further degrade growth and reproduction of individuals.

We describe the details necessary to adapt the model to the right whales and estimate the parameters in the Appendix. The parameter values are listed in Table 2.4.

\subsection{Results}

\subsubsection{Growth and reproduction}

To investigate the dependence of growth and reproduction on energy intake in right whales, we look at the growth and reproduction in a constant environment, and in- 
vestigate the consequences of seasonal fluctuations and starvation. Unless otherwise noted, all plots are of a first-generation, first-born individual. This is necessary because our model needs energy input during gestation and nursing of one generation, which requires a mother from a prior generation. We simulate the zero-generation mother by initializing the model from her weaning. We used the whale MH-89424-Eg from Moore et al. (2005) to estimate her initial conditions $\left(V(0)=1 \mathrm{~m}^{3}\right.$, $E_{L}(0)=1.86 \cdot 10^{6} \mathrm{kcal}, E_{B}(0)=1 \mathrm{kcal}$, no burden $)$.

To investigate growth, we calculate the length of a non-reproducing individual as a function of age for values of the scaled functional response $f$, a measure of energy availability defined by equation (2.5), ranging from $f=0.75$ to $f=1$ (Figure 2-2). The data from Moore et al (2005) for individuals older than 1 year fall within the sizes predicted for the range in $f$. Using (2.6), the observed ultimate size of about $14.5 \mathrm{~m}$ suggests that an appropriate value of $f$ for the North Atlantic right whale would be around 0.8. This is an under-estimate, as it does not take into the account energy spent on reproduction.

To account for the energy spent on reproduction, we use observed calving interval of about five years (Kraus et al. 2001) to estimate $f$ in the North Atlantic, $f_{N A}$. Comparing the mean interval between reproductive events of a first-generation mother over a 100-year period for a range of energy availability (Figure 2-4). Comparison between the calculated and observed calving intervals suggests that $f_{N A}=0.9$. A reproductively active female experiencing $f_{N A}$ grows to the same size as a nonreproducing female experiencing $f=0.8$ (Figure 2-3). Thus we set $f=0.9$ in all simulations unless otherwise noted.

According to the model, an increase of only $10 \%$ in $f$, representing an order of magnitude increase in $e_{I}$ for the given (underestimated) $I_{\max }$, would decrease the calving interval of the North Atlantic right whales to three years, equal to that of their southern cousins. Furthermore, the age at first parturition, which includes the gestation period of the first calf, decreases from the predicted seven years to six years 


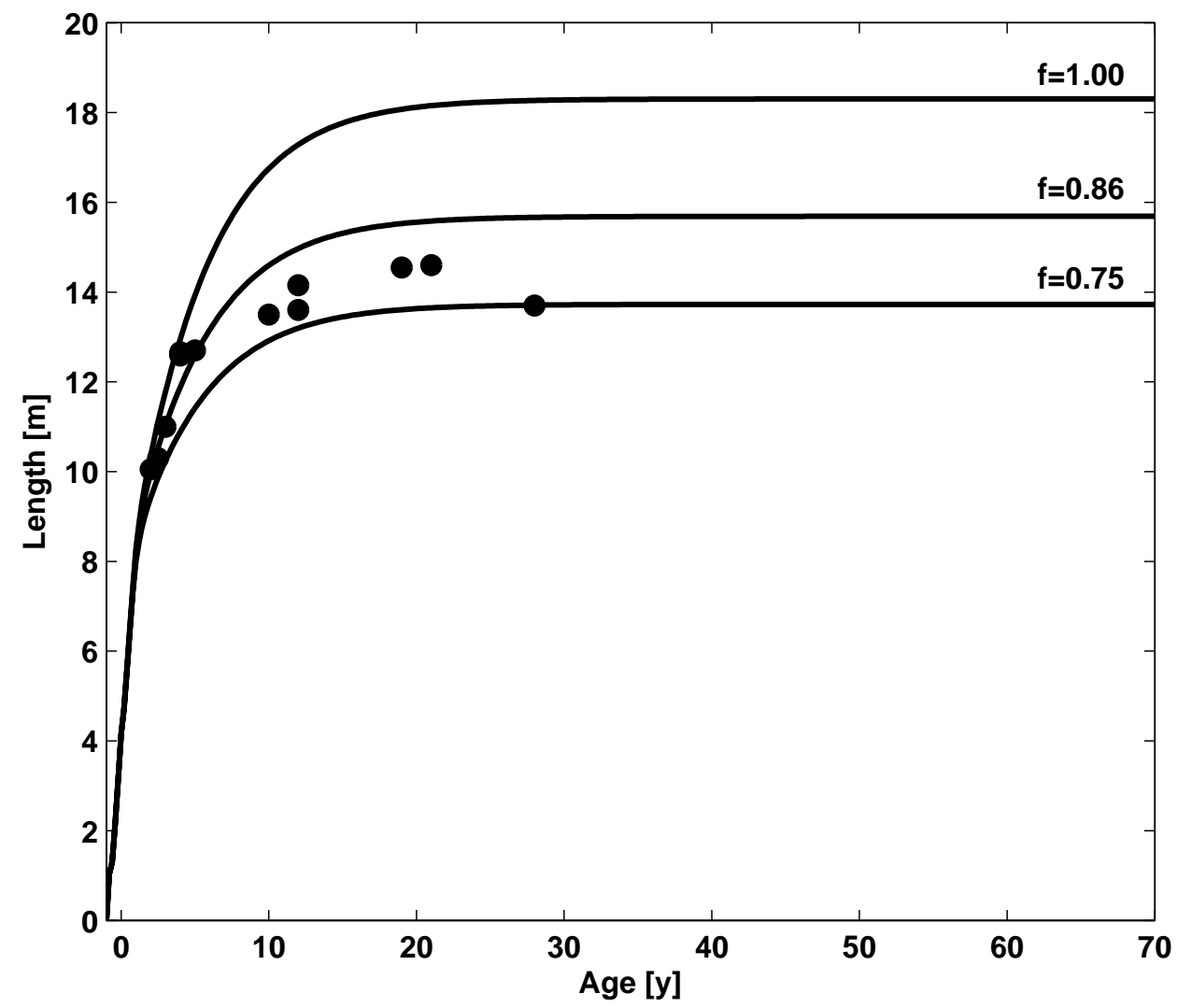

Figure 2-2: Length of non-reproducing right whales as a function of age for a range of $f$. Circles represent data for individuals older than one year from Moore et al (2005). Negative ages represent gestation. 


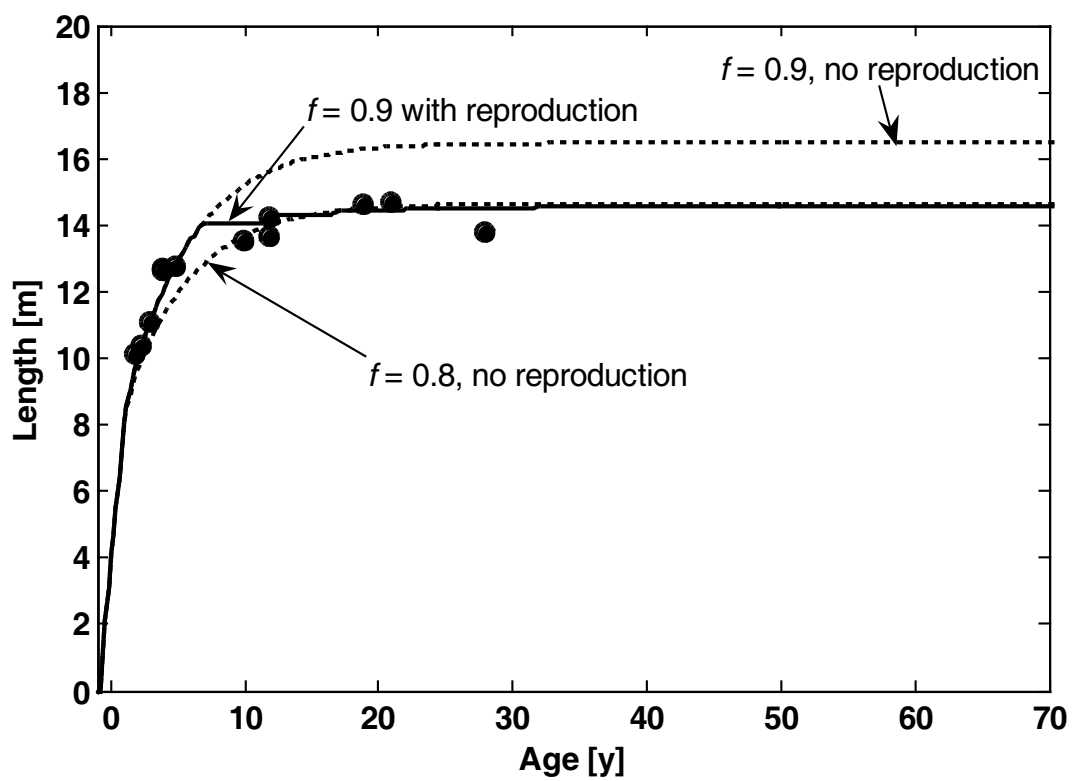

Figure 2-3: Influence of reproduction on growth. Reproducing females (solid line) experiencing $f=0.9$ grow to the same size as non-reproducing females (dotted lines) experiencing $f=0.8$. 
for the same change in $f$.

A whale's response to seasonal environmental variability may influence reproduction. The energy availability, $f$, is a Type II functional response of $e_{I}$, the energy density available in the environment (see equations 2.4 and 2.5) which, in turn, depends on the season and the location of the right whale. Rather than trying to capture the intricate and fairly poorly understood typical yearly energy availability pattern of the North Atlantic right whales (see Winn et al. 1986 and The North Atlantic right whale recovery team 2000), we assumed that the energy density in the environment experienced by the individuals oscillates sinusoidally. This corresponds to the assumption that there is a season of food abundance, a season of food scarcity, and two transitional seasons. Since the functional response $f$ is determined by the ratio of $e_{I}$ and the half-saturation constant $K_{I}$, we did not have to determine $K_{I}$ explicitly. Instead, we wrote $f$ in terms of $e_{I} / K_{I}$. Then, inserting the sinusoidal environmental forcing, $e_{I} / K_{I}=\alpha(1+\sin 2 \pi(t+\phi))$, and rearranging gives:

$$
f(t)=\frac{\alpha(1+\sin 2 \pi(t+\phi))}{1+\alpha(1+\sin 2 \pi(t+\phi))},
$$

where $\phi$ is the phase shift of the sinusoidal relative to breeding season, and $\alpha$ the amplitude of oscillations. For each simulated $\alpha$, we calculated average food availability, $f_{\alpha}=\int_{0}^{1} f(t) d t$, and compared first parturition times and calving intervals to those of constant food availability $f=f_{\alpha}$ (Figure 2-4). We use $\phi=0.5$ y in the simulations, corresponding to the assumption that mothers give birth at the onset of food scarcity. This assumption is consistent with the observations (Winn et al. 1986). When the onset of food abundance happens at the start of the breeding season $(\phi=0 \mathrm{y})$, first parturition times and calving intervals are significantly longer for low $f_{\alpha}$. Generally, seasonal oscillations increase the calving interval and time to maturity (Figure 2-4), but the effect is small for large $f$.

The energy budget of individuals changes during growth and reproduction. An individual has the largest energy storage density $\left(e_{L}=E_{L} / V\right)$ at weaning (Figure 2-5 


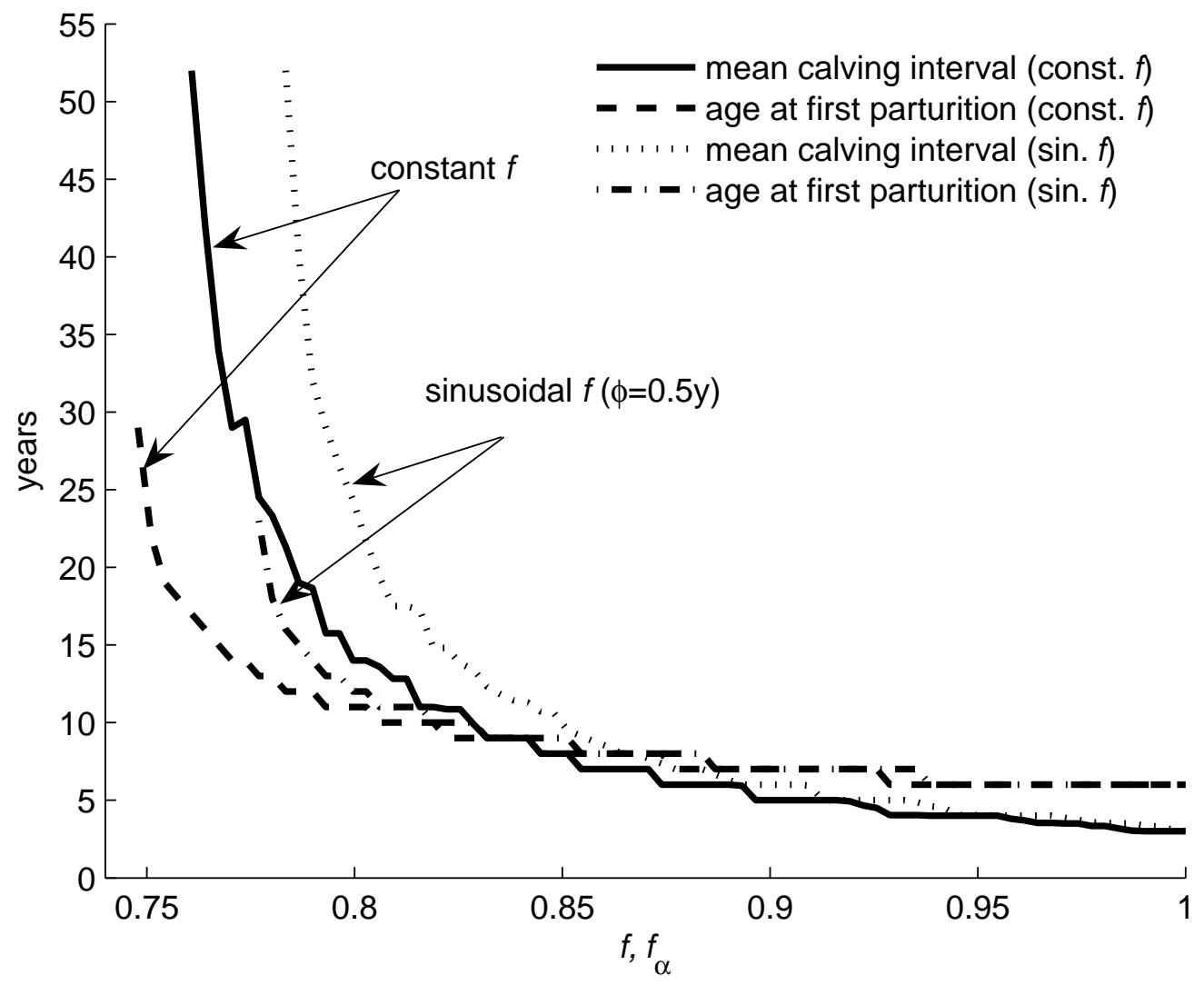

Figure 2-4: Calving interval averaged over simulation time, and age to maturity for a range of average energy availability $(f)$. The seasonally variable $f(t)$, described by (2.23), has an average of $f_{\alpha}$ and a period of a year (see text for discussion). 

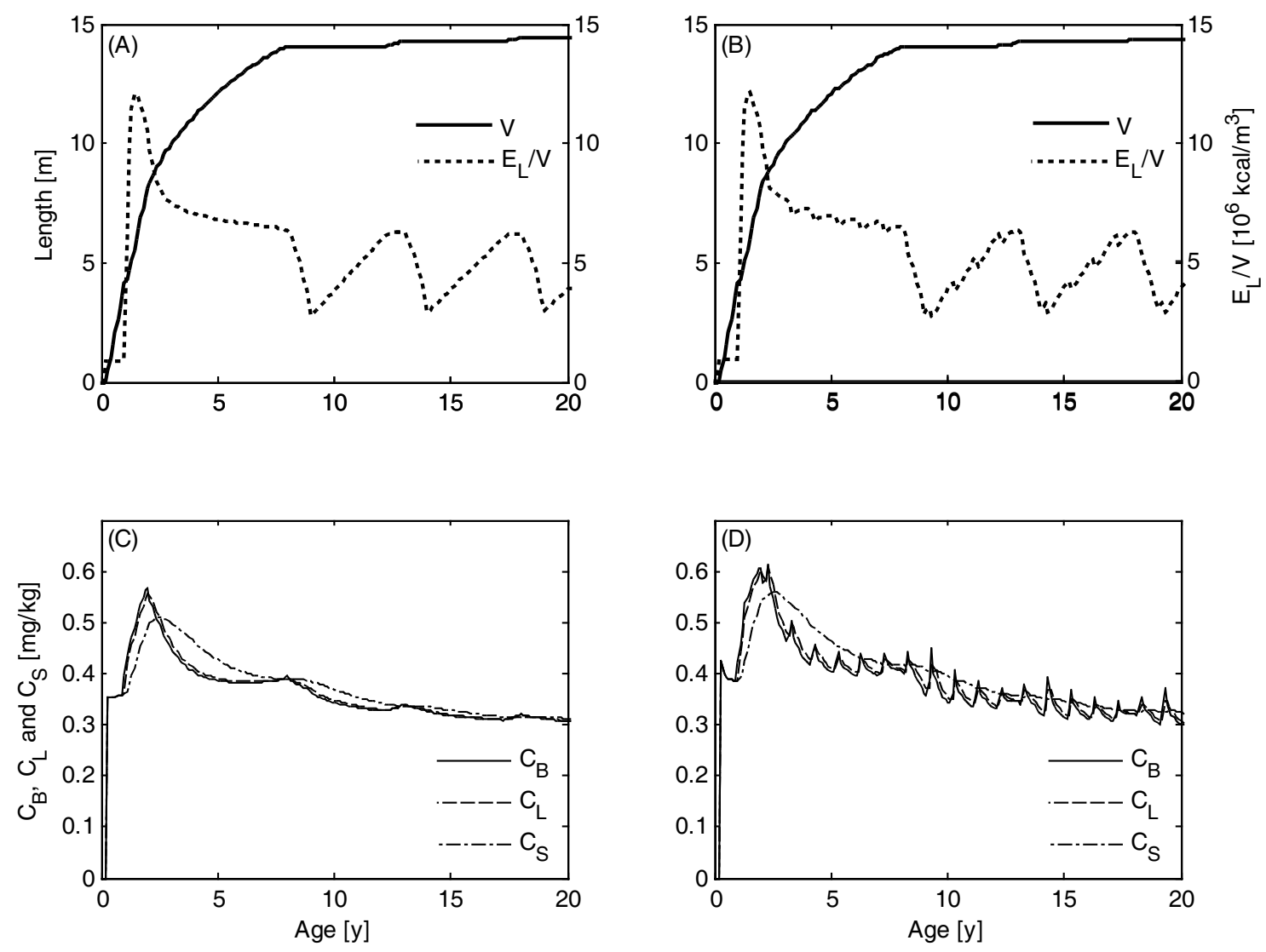

Figure 2-5: Energy and toxicant distribution for a female in a constant (plots (A) and (C)) and fluctuating (plots (B) and (D)) environment. The energy assimilation in the fluctuating environment is described by (2.23) and has an average of $f_{N A}$. Plots (A) and (B): length and energy storage density. Plots (C) and (D): concentration of toxicants in the blood $\left(C_{B}\right)$, lipid energy storage $\left(C_{L}\right)$ and structural lipids $\left(C_{S}\right)$, in (mg toxicant/kg lipid). 
,(A) and (B)). This surplus energy gets utilized for growth after weaning; the growth rates decrease once that additional energy received from the mother is depleted. The model predicts that reproductively active females are smaller than males of the same age because females stop growing during reproductive events (Figure 2-5 (A)). Reproductive signal is noticeable even in the fluctuating environment, with the females spending about $55 \%$ of their energy storage on reproduction when $f=f_{N A}$, and only about $39 \%$ when $f$ is $10 \%$ higher. Therefore, a relatively small increase in energy intake $\left(F_{I B}\right)$ not only substantially decreases the calving interval, but also reduces the stress (in terms of energy loss) on the mother as well. Consistent with observations (Moore, personal communication), the model predicts that an adult male dies of complete starvation (e.g. because it cannot feed due to entanglement in fishing gear) in a little less than 8 months (not shown).

An interesting consequence of the dynamic energy budget predicted by the model is the possibility of a calving interval hysteresis: the calving interval depends not only on energy availability, but also on the history of energy availability. If there is a longterm decrease in $f$, the calving interval of females that have grown up during higher $f$ will be longer than that of females which have matured during lower $f$. For example, if $f$ decreases from $1.1 f_{N A}$ (three year calving intervals) to $f_{N A}$ when the female is 20 years old, her average calving interval increases to 6 years, rather than 5 years, as it would be had she experienced $f_{N A}$ all of the time. This means that, depending on its duration, high energy availability could have negative long term consequences on a population if it is followed by a stretch of low energy availability because it may take a whole generation until the population optimally utilizes the lower energy availability. Furthermore, when the energy availability is extremely low, smaller mature females are able to take better advantage of a sudden increase in energy availability. Both of these effects are a consequence of higher maintenance requirements of larger females. When the energy is readily available, bigger size is advantageous because it helps take advantage of the available energy, but when the energy is scarce, smaller size is more 
desirable because of lower maintenance costs.

\subsubsection{Toxicant distribution and vertical transfer}

Energy dynamics drives bioaccumulation and distribution of toxicants. We assumed that toxicants are introduced into the organism exclusively through energy assimilation, excreted exclusively through reproduction, and biotransformed exclusively in the blood compartment. Initially, we ignore biotransformation $\left(\gamma_{B}=0\right)$.

When energy and toxicant in the environment are constant, concentrations of toxicants in all types of lipid follow a similar pattern of bioaccumulation (Figure 2-5, (C)). During nursing, nurslings bioaccumulate toxicants rapidly because they ingest milk with high concentration of toxicants, use some of the energy from the milk for maintenance and growth, but have no way of excreting the toxicants. Toxicant concentrations of the calves peak at weaning and then decrease due to dilution of toxicants by ingestion of lipids with relatively low environmental toxicant concentrations.

Energy budget dynamics in a variable environment result in toxicant concentration differences between compartments (Figure 2-5, (D)). When the energy assimilation rate is high, the organism stores the ingested lipids and dilutes the toxicants in the blood, as well as in the lipid energy storage. When the energy assimilation is low, the organism is starving and drawing lipids and toxicants from the lipid energy storage. Since lipids are used for maintenance, toxicants accumulate in the blood. This starvation-induced mobilization of toxicants is clearly visible as peaks of concentration in blood and lipid energy storage. As $f$ oscillates, the concentrations in the blood and the lipid energy storage follow with a phase lag. The phase lag of concentration oscillations in the blood is about a month less than that of lipid energy storage. Due to the diffusive nature of exchange of toxicants between the structural and energy storage lipids, structural lipids act as a low-pass filter: since $C_{S}$ always tends to

equilibrate with $C_{L}$, but does so slowly, $C_{S}$ reflects only trends in $C_{L}$. Complete starvation (e.g. due to entanglement in fishing gear) can increase $C_{B}$ by an order of 
magnitude (not shown).

After the females mature, they export toxicants through reproduction. Females are predicted to lose about $40 \%-45 \%$ of their toxicant burden during a reproductive event, consistent with about a $53 \%$ loss estimated during 18-months of nursing in beluga whales (Hickie et al. 2000). Reproduction is not completely efficient because mothers discard of tissue (e.g. placenta) and a proportion of the mother's milk is excreted by the calf. These inefficiencies (parameterized by $k_{R}$ ) imply that calves assimilate only $70 \%$ of the burden lost by the mother, or about $30 \%$ of mother's initial burden.

The bulk of (potential) decrease in concentration of the toxicants in the mother's tissue comes from dilution after the reproduction event, rather than loss of toxicants during reproduction. During reproduction, the energy transferred has almost the same concentration of toxicants as the lipid storage. Therefore, the concentration of toxicants in all the mother's compartments is roughly constant for the duration of the reproductive event. After the reproductive event, the mother ingests and stores lipids from the environment with a lesser toxicant concentration than her own, thus diluting the toxicant and reducing the concentration in her lipids. This may not happen when the energy availability is low and the rate of bioaccumulation is greater than the rate of dilution.

For a grown female in a constant or seasonally varying environment, the export of toxicants during reproduction and the bioaccumulation between two reproductive events effectively equilibrate after a few reproductive events. The export is larger the greater the burden, while bioaccumulation between two reproductive events remains constant. Hence, if the export during a reproductive event is greater than the toxicants accumulated between two reproductive events, females experience a reduction of their toxicant burden. If the export is smaller than the bioaccumulation, the burden increases. Eventually, the two are practically equal. Hence, in the long run, the toxicant transfer is determined by the difference between bioaccumulation and 
reproductive loss. The mother's history of pre-reproductive exposure is, therefore, reflected only in the first few reproductive events, and the transfer of toxicants to the next generation after those few events is practically the same regardless of the mother's pharmacokinetic history. In Figure 2-5 (C), toxicant transfer is close to equilibrating by the third or fourth reproductive event.

The calculated pattern of bioaccumulation is consistent with the commonly assumed marine mammal patterns and observed PCB concentrations in North Atlantic right whales and other marine mammals (Lee et al. 1996, Ross et al. 2000, Weisbrod et al. 2000 (Figure 2, top right plot)). Weisbrod et al (2000) measured lipidnormalized prey concentrations of PCBs between $0.01 \mathrm{mg} / \mathrm{kg}$ and $0.4 \mathrm{mg} / \mathrm{kg}$, and the right whale blubber concentrations between 0.1 and $8 \mathrm{mg} / \mathrm{kg}$. This suggests that bioaccumulation amplifies the environmental concentration by an order of magnitude, consistent with our predictions.

Even though the accumulation of toxicants in both males and females is greater in seasonally variable environments, there are significant differences between male and female patterns of accumulation (Figure 2-6). For example, a 30-year old male is larger than a female of the same age and has more than double the concentration of toxicants. The large difference between toxicant concentrations in male and female right whales can only be attributed to vertical toxicant transfer from the mother to her calf during gestation and lactation.

The mass of toxicant transferred correlates with the calving interval (especially for second- and later- born calves) and depends on the birth order of the calf (Figure 2-7). For large energy availability, the firstborn calf can get as much as twice the burden the subsequent calves get because its mother accumulated a large burden through nursing and maintenance requirements during nursing. However, if food is low, the calving interval is large and the toxicant has an opportunity to bioaccumulate to a greater extent in the interval between the calves than before the first calf. Then, the transfer of toxicants increases with birth order. For the values of $f$ currently 


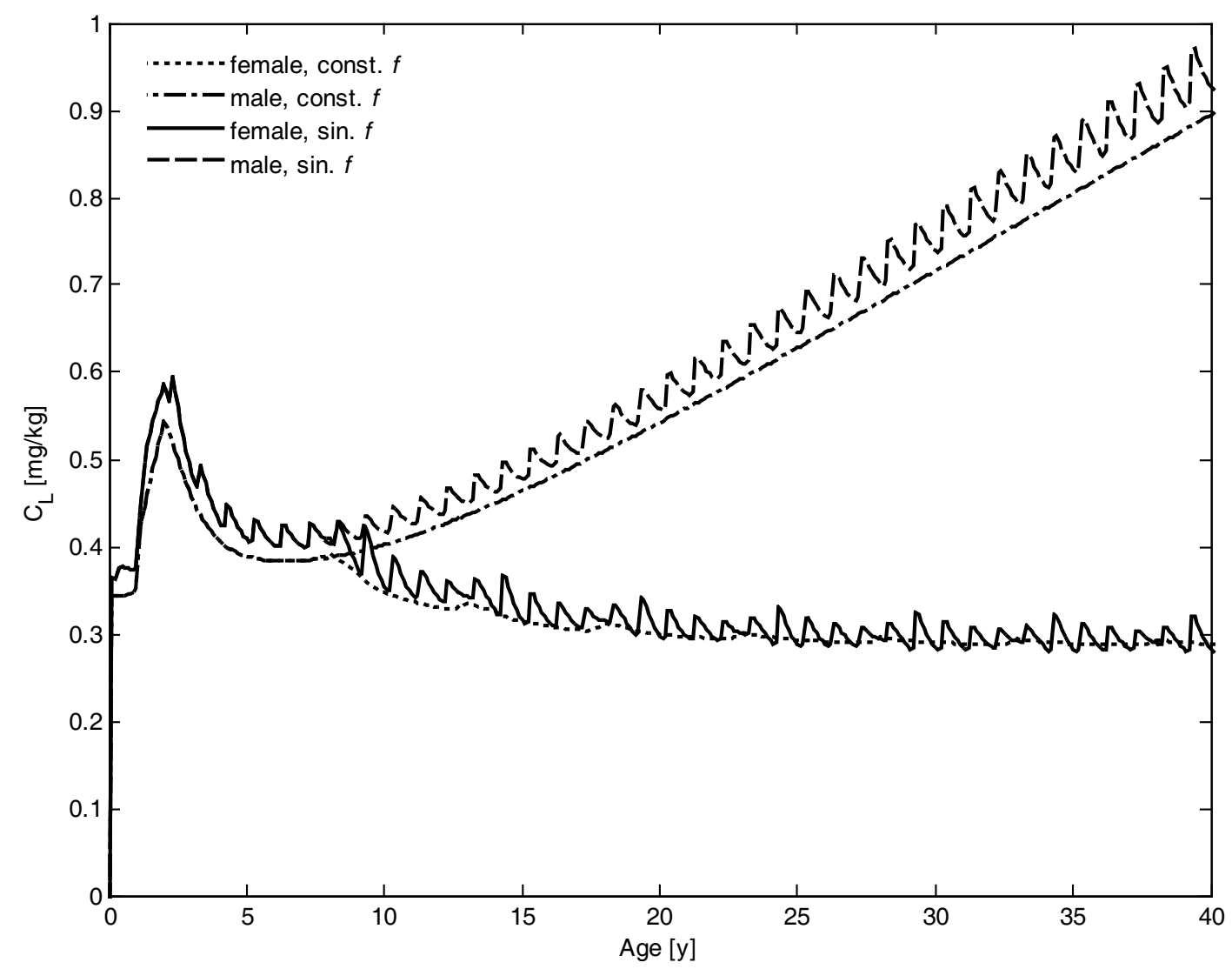

Figure 2-6: Male and female right whale toxicant bioaccumulation in constant and seasonal environments. $f=f_{\alpha}=f_{N A}$. 


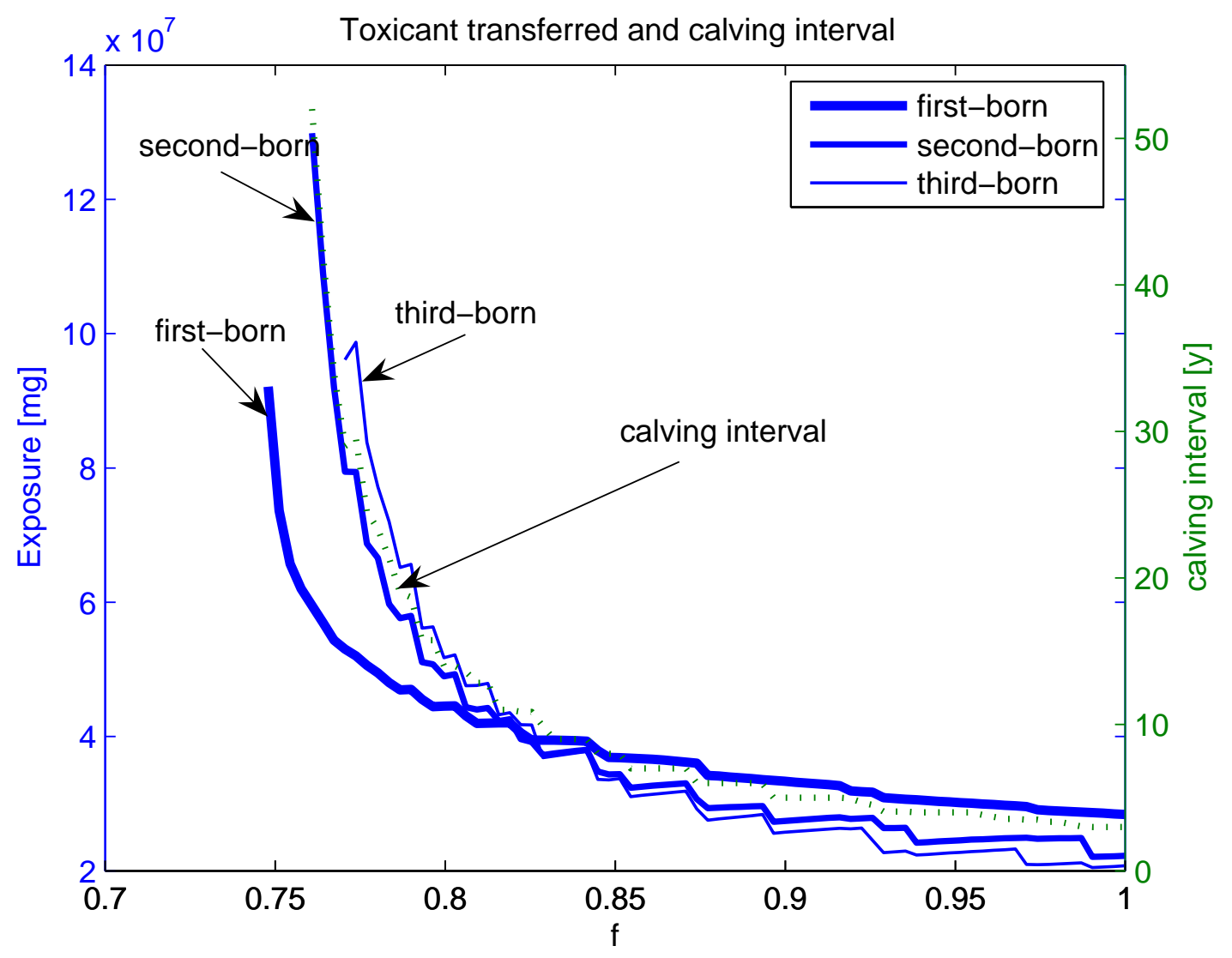

Figure 2-7: Toxicant transferred to first-, second- and third-born for a range of energy availabilities. 
experienced by the right whales, toxicant transferred decreases with birth order.

Because there were no data available, we assumed a low but arbitrary proportion of structural blubber $\left(e_{S 0}\right)$, and for simplicity we set the rate of biotransformation of toxicants $\left(\gamma_{B}\right)$ to zero in our simulations. To better understand how these parameters influence the analyses, we repeated simulations for a range of values of $e_{S 0}$ and $\gamma_{B}$.

The proportion of structural blubber does not significantly influence time to maturity, calving interval, or vertical transfer of toxicants when structural lipids constitute less than $5 \%$ of the total lipids $\left(e_{S 0}<510^{5} \mathrm{kcal} / \mathrm{m}^{3}\right)$. The effects are moderate when the structural lipids account for up to $13 \%$ of the total lipids $\left(e_{S 0}<10^{6} \mathrm{kcal} / \mathrm{m}^{3}\right)$ : the age to maturity increases by a year because more lipids have to be accumulated prior to reproduction, and concentrations of toxicants decrease by $50 \%$ because a greater proportion of the body is in the form of lipids. Consequently, the vertical transfer to the first three calves decreases, but by the fourth calf, transfer effectively equilibrates with bioaccumulation and is the same as if we ignored structural lipids.

Small $\gamma_{B}$ does not perceptibly influence the analyses. The estimates of the biotransformation rates of PCBs are low: $0.05-0.08 \mathrm{y}^{-1}$ in beluga whales (Hickie et al. 1997), and $0.2-0.4 \mathrm{y}^{-1}$ in humans (Phillips et al. 1989). The individual toxicant concentrations and the vertical toxicant transfer are nearly linear functions of $\gamma_{B}$ and

environmental toxicant concentration $\left(C_{I}\right)$, even when $\gamma_{B}$ is as large as $5 \mathrm{y}^{-1}$ (Figure 2-8). At rates of biotransformation comparable to those of PCBs, individual toxicant concentrations and toxicant transfer are practically the same as those without biotransformation. Even biotransformation rates on the order of months $\left(\gamma_{B} \approx 10\right)$ change the bioaccumulation and toxicant transfer by less than $50 \%$.

\subsection{Discussion}

Understanding the processes of accumulation, partitioning and vertical transfer of toxic substances is a necessary step towards quantifying impacts of exposure to con- 


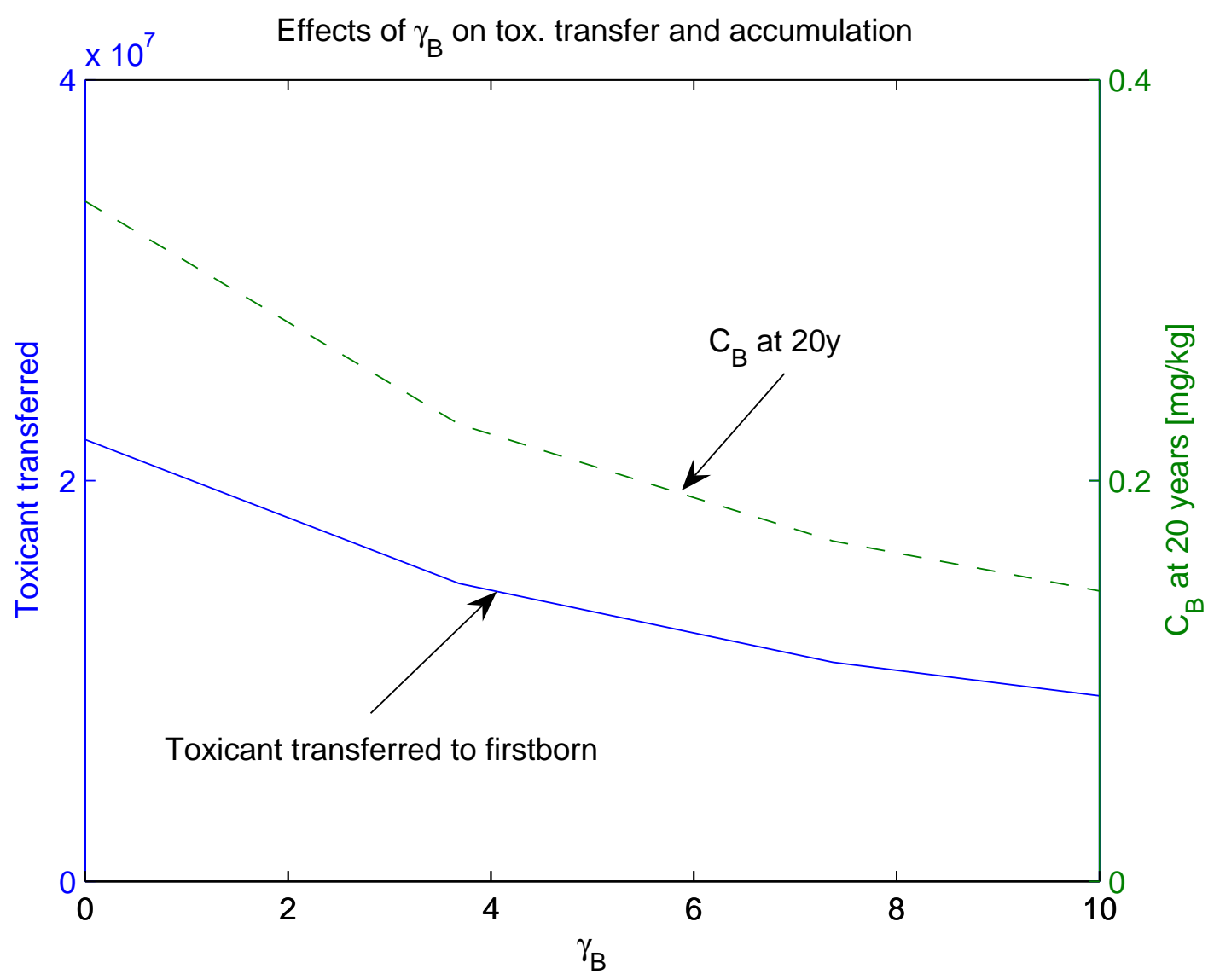

Figure 2-8: Plot of toxicant transferred to the firstborn (left axis) and $C_{B}$, the toxicant concentration in the blood of a reproducing female at 20 years of age (right axis) for $\gamma_{B}$ ranging from 0 to 5 . Toxicant transfer to later born calves follow the same trend as the firstborn, and toxicant concentrations at other ages follow the same trend as the one for 20 years of age. 
taminants on individuals and, in turn, populations. The lipids are by far the largest pool of energy, the largest storage depot of lipophilic toxicants, and the main vector of vertical toxicant transfer in marine mammals. Our model predicts the storage and utilization of lipids for a given energy intake, and calculates the associated toxicant dynamics. For a specified food availability and lipid-normalized concentration of toxicants in the environment, it predicts the size and energy reserves of an individual as a function of age, and the lipid-normalized concentrations of toxicants in the three main reservoirs: blood, lipid energy storage, and structural lipids. When applied to the right whale, the model captures many life history parameters, such as age to maturity, calving intervals and the dynamics of starvation, remarkably well. The approach - and most of the results - are applicable to other marine mammals and, more generally, other mammalian species that utilize mostly lipids for energy storage. Our most important results were:

1. The typical energy availability experienced by the right whales (estimated from observed calving intervals), leads to a first parturition time of seven years for the North Atlantic, and six years for the southern right whales.

2. A difference in feeding rates (characterized by the model parameter $f$ ) of only $10 \%$ accounts for the difference in first parturition times and calving intervals between North Atlantic and southern right whales.

3. Seasonal variability significantly increases age at first parturition and calving intervals at low values of $f$, but has a very limited effect for large values of $f$.

4. At low $f$, the timing of seasonal variability relative to reproductive season influences the maturation time and calving interval.

5. Reproduction depends on past, as well as current energy availability (see the discussion on the calving interval hysteresis in the Results section). This is partly because we assume that growth is limited by the ability to meet main- 
tenance requirements, rather than genetics. Calving hysteresis depends on the degree to which this assumption holds for a particular species.

6. Lower energy availability increases the toxicant concentrations and vertical transfer of toxicants.

7. Contrary to expectations (e.g. Aguilar and Borrel 1994, Hickie et al. 2000, Wells et al. 2005), the firstborn calf does not necessarily receive the greatest burden. Energy availability determines the balance between bioaccumulation and dilution-by-growth of the mother's lipid energy storage after weaning, thus determining the relationship between birth order and burden received.

8. Biotransformation does not significantly influence toxicant concentrations and vertical transfer of persistent lipophilic toxicants (such as PCBs).

9. Right whale mothers lose about $40-45 \%$ of their toxicant burden during a reproductive event, and right whale calves assimilate about $30 \%$ of their mother's burden during gestation and nursing.

The quantitative predictions of results 1,2 and 9 are specific to right whales, but they suggest that small changes in energy availability could have a big impact on reproduction of any marine mammal whose reproduction is limited by the food supply. Further reductions in food supply expose them to additional risks: increased toxicant exposure (result 6), increased exposure with birth order (result 7), decreased ability to buffer seasonal fluctuations (result 3) and increased susceptibility to temporal shifts in peak food availability (result 4).

The time scales at which lipids respond to environmental forcing have implications for sampling procedures. Blubber biopsies mainly include energy storage lipids, but can include a significant portion of structural lipids as well (Aguilar and Borrell 1990). Since the concentration in the blood during starvation increases more rapidly than the concentration in the energy storage lipids, measuring toxicant concentration in 
energy storage lipids can underestimate the toxicant concentration in the blood and the resulting organ exposure. This underestimate can be exacerbated if the biopsy includes a significant proportion of structural lipids because they are even slower to react to changes of concentrations in the blood.

The biotransformation of persistent toxicants can be ignored in some analyses (result 8), but if the metabolites are responsible for the toxic effect, the analysis may require inclusion of biotransformation. If the dynamics of the metabolites are important, another compartment with the metabolites as a state variable should be added to the model.

According to our model, individuals grow larger and reproduce more frequently when food is more abundant. The calving hysteresis (result 5) suggests that growing during times of abundance may not increase reproduction in the long run if the periods of abundance are short and infrequent. Therefore, losing the ability to grow at a mature age may result in more offspring: although organisms are not able to fully utilize years of abundance because of their smaller size, they make up for it during the times of scarcity. In such environments, cessation of growth may offer a competitive advantage over indeterminate growth.

It is advantageous to give birth at the onset of seasonal food scarcity (result 4). This contrasts with organisms that benefit from abundance at the earliest stages of the development (Klanjscek et al, 2006). Further research could help explain the timing of reproduction of marine mammals relative to seasonal cycles of food.

Linking observables such as copepod density to $e_{I}$ and the energy intake is a daunting task, but our analyses do not depend on the correct interpretation of $e_{I}$ because we were concerned with the energy intake, which is a linear function of $I_{\max }$ and $f$. Therefore, a small underestimate of $I_{\max }$ can be compensated for by a small overestimate of $f$. Translating the differences in $f$ into differences in $e_{I}$, however, highly depends on the value of $I_{\max }$. Our current estimate of $I_{\max }$ implies that $e_{I}$ experienced by the North Atlantic right whale is about an order of magnitude lower 
than $e_{I}$ experienced by the southern right whale. Even though such differences in copepod densities are often observed (Beardsley et al. 1996, Mayo and Marx 1990, Wishner et al. 1988, Baumgartner et al. 2003), they cannot be directly translated into changes in $e_{I}$ because these changes depend on the value of other energy intake parameters. For example, our estimate of $e_{I}$ comes from $f$ of 0.9 in the Atlantic, and 0.99 in the southern seas. If $I_{\max }$ were $10 \%$ higher, $f$ experienced by the Northern Atlantic right whale would have been 0.82 , and that of the southern whale 0.9 - still a $10 \%$ difference in $f$, but only a two-fold difference in $e_{I}$ due to the nonlinearity of the functional response. Therefore, the interpretation of $e_{I}$ depends on the estimate of $I_{\max }$ and $k_{I}$. To better estimate these parameters, we would need to incorporate variable costs of foraging, and much more information on spatially explicit copepod dynamics and right whale distribution than is available at this time. Alternatively, given a population model based on this individual model, we could fit these parameters to observations of right whale population dynamics and copepod abundance.

The calculated ages to first parturition of seven and six years, for North Atlantic and southern populations respectively, are significantly smaller than estimates of $9.5 \pm$ 2.32 years for the North Atlantic (Kraus et al. 2001) and 8.5 \pm 2 years for the southern right whales (Best et al. 2001). However, the average estimates may be inflated by variable environmental conditions, miscarriages, or lack of fertilization, none of which are included in the simulations; ages at first parturition as low as five years have been observed in the North Atlantic (Knowlton et al. 1994).

Our model can help determine the reproductive costs of anthropogenic feeding interruptions. This could help guide the policy on whale watching, and the use of alarms to reduce ship strike mortality by inducing collision-avoidance responses in the whales (Nowacek et al. 2004). The reduction in energy intake due to feeding interruptions can be represented by reducing $I_{\max }$. Reducing $I_{\max }$ of Northern Atlantic right whales by only $16 \%$ is equivalent to reducing $f$ to 0.75 , making reproduction impossible (Figure 2-4). Quantifying these costs of feeding interruptions could help 
balance them with economic and demographic benefits of feeding interruptions.

Adapting the model presented in this paper to other marine mammal species involves adapting the structure of the energetics and the pharmacokinetics parts of the model, linking them, and estimating the parameters. Blood, structure and energy reserves are crucial to the formulation of the energetics part of the model. We linked the two parts of the model assuming that lipid dynamics drives toxicant transport. For lipophilic toxicants, this may be sufficient; for others, different approaches possibly even additional compartments - may be necessary. Additional compartments are necessary to distinguish between types of lipids. The need to do so, however, depends on the significance of the different types of lipids in the particular species, and toxicological questions of interest. Our results suggest that, to predict the patterns of bioaccumulation and vertical transfer, structural lipids can be omitted if they constitute less than $5 \%$ of the total lipids.

Estimating the percentage of structural lipids is difficult. Starvation studies on Harbor Porpoises (Phocoena phocoena) suggest that less then half of the lipids are readily metabolized (Koopman et al. 2002). This does not imply that all the remaining blubber is structural because death by starvation happens when the flux from the energy reserves cannot meet maintenance; the flux becomes insufficient before the reserves disappear. Depending on its physiology (e.g. if $\beta_{L}$ or $k_{L}$ is low), an animal can die of starvation with ample reserves left.

Blubber morphology can help distinguish structural from energy storage blubber. For example, high proportion of collagen in blubber (Pond 1987), low vascularization (Struntz et al. 2004), and negligible responses to physiological condition of the animal (Aguilar and Borrell 1990, Koopman et al. 2002) suggest structural blubber. The overall proportions of structural and energy storage lipids are, however, largely unknown.

Running the model requires all parameters listed in the Table 2.4, which can be estimated in many ways. Rather than tuning the parameters to fit the outputs 
of the model to observations, we estimated them using physiological considerations and morphometric data. To do that, we needed to derive and rely heavily on the relationship between length and structural volume of right whales. This relationship may be a good approximation for other species, but we believe the parameters in the relationship are species-specific. Likewise, some estimates $\left(\beta_{L}, \beta_{G}, g, \gamma_{B}\right.$ and $\left.D_{L S}\right)$ may hold for most marine mammals, but the rest are probably species-specific. There is a theory that characterizes interspecific variation in model parameters for simpler energy budget models (Kooijman 2000). A challenge for theorists is to develop analogous insight applicable to more complex models, like ours, that share many assumptions with their simpler counterparts.

Our model describes the responses of individuals, not populations, to environmental fluctuations. Nevertheless, the conclusions have implications for populations. For example, if energy availability is low for a long time and then increases, a baby boom can be expected. Greene et al. (2003) observe such correlations (see also Kenney et al. 2001), and suggest that the North Atlantic oscillation (NAO) is the main predictor of calving success (see also Fujiwara and Caswell 2001). Our model provides a mechanistic link between the environment and the individual, but needs a population model to investigate consequences on the population dynamics.

Similarly, when toxicant concentrations fluctuate, bioaccumulation, vertical transfer, and export of toxicants out of the population through death may influence the exposure of individuals. Quantifying that response, however, requires a population model in conjunction with a toxicant action model to account for effects of exposure on individuals. These effects can be included through exposure-dependent modifications of model parameters, for example through foraging ability or maintenance costs (Nisbet et al. 1997). We are formulating population models based on the individual model presented here to address such questions. 


\subsection{Acknowledgements}

We thank Michael Moore for lending us his expertise on marine mammal life history and Mark Hahn for his insights in toxicology. We also thank Erik Noonburg for insights on dynamic energy budget models, Mark F. Baumgartner and Peter Tyack for their help with marine mammal energetics, and Eric Montie for discussions on blubber function and morphology. This research was supported by the David and Lucile Packard Foundation, the US National Science foundation (DEB-9973518 and OCE-0083976), the US Environmental Protection Agency (R-82908901-0), and the WHOI/MIT Joint Program in Oceanography.

\subsection{References}

Aguilar, A., and A. Borrell. 1990. Patterns of lipid content and stratification in the blubber of fin whales (Balaenoptera physalus). Journal of Mammalogy 71:544-554.

Aguilar, A., and A. Borrell. 1991. Heterogeneous distribution of organochlorine contaminants in the blubber of baleen whales: Implications for sampling procedures. Marine Environmental Research 31:275—286.

Aguilar, A., and A. Borrell. 1994. Reproductive transfer and variation of body load of organochlorine pollutants with age in fin whales (Balaenoptera physalus). Archives of Environmental Contamination and Toxicology 27:546 - 554.

Aguilar, A., A. Borrell, and T. Pastor. 1999. Biological factors affecting variability of persistent pollutant levels in cetaceans. Journal of Cetacean Research and Management Special Issue 1:83-116.

Angell, C. 2005. Body fat condition of right whales, Eubalaena glacialis and Eubalaena australis. Ph.D. thesis, Boston University, Boston, MA

Baumgartner, M. F., and B. R. Mate. 2005. Summertime foraging ecology of North Atlantic right whales. Marine Ecological Progress Series 264:123-135.

Baumgartner, M. F., T. V. N. Cole, R. G. Campbell, G. J. Teegarden, and E. 
G. Durbin. 2003. Associations between north Atlantic right whales and their prey, Calanus finmarchicus, over diel and tidal time scales. Marine Ecological Progress Series 264:155-166.

Beardsley, R. C., A. W. Epstein, C. Chen, K. F. Wishner, M. C. Macaulay, and R. D. Kenney. 1996. Spatial variability in zooplankton abundance near feeding right whales in the great south channel. Deep-Sea Research Part II, Topical Studies in Oceanography 43:1601-1625.

Best, P. B. 1994. Seasonality of reproduction and the length of gestation in southern right whales Eubalaena australis. Journal of Zoology 232:175-189.

Best, P. B., and D. M. Schell. 1996. Stable isotopes in southern right whale (Eubalaena australis) baleen as indicators of seasonal movements, feeding and growth. Marine biology 124:483-494.

Best, P. B., A. Brandao, and D. S. Butterworth. 2001. Demographic parameters of southern right whales off south Africa. Journal of Cetacean Research and Management, Special Issue 2:161-169.

Boon, J., I. Oostingh, J. van der Meer, and T. Hillebrand. 1994. A model for the bioaccumulation of chlorobiphenyl congeners in marine mammals, European Journal of Pharmacology: Environmental Toxicology and Pharmacology, 270:237-251.

Boon, J. P., E. van Arnhem, S. Jansen, N. Kannan, G. Petrick, D. Schulz, J. Duinker, P. Reijnders, and A. Goksoyr. 1992. The toxicokinetics of PCBs in marine mammals with special reference to possible interactions of individual congeners with the cytochrome P450-dependent monooxygenase system: an overview. Pp. 11960, in C.H. Walker and D.R. Livingstone, editors. Persistent Pollutants in Marine Ecosystems. Pergamon Press, Oxford.

Borga, K., and A. Di Guardo. 2005. Comparing measured and predicted PCB concentrations in Arctic seawater and marine biota. Science of the Total Environment $342: 281-300$.

Borga, K., A. Fisk, P. Hoekstra, and D. Muir. 2004. Biological and chemi- 
cal factors of importance in the bioaccumulation and trophic transfer of persistent organochlorine contaminants in Arctic marine food webs. Environmental Toxicology and Chemistry 23:2367-2385.

Brody, S. 1968. Bioenergetics and Growth. Hafner Publishing Company Inc. New York.

Bronson, F., and J. Manning. 1991. The energetic regulation of ovulation: a realistic role for body fat. Biology of Reproduction 44:945 — 950 .

Brownell Jr, R. L., P. J. Clapham, T. Miyashita, and T. Kasuya. 2001. Conservation status of North Pacific right whales. Journal of Cetacean Research and Management, Special Issue 2:269-286.

Brunell, S. R. 2001. Aspects of the reproductive biology, movement and site fidelity of right whales off Australia., Journal of Cetacean Research and Management, Speical Issue 2:89-99.

Convention, W. 1931. Convention for the regulation of whaling, Geneva. League of Nations Treaty Series 155:349-365.

Crank, J. 2004. The Mathematics of Diffusion, second edition, Oxford University Press.

De Swart, R. L., P. S. Ross, L. J. Vedder, H. H. Timmerman, S. Heisterkamp, H. Van Loveren, J. G. Vos, P. J. H. Reijnders, and A. D. M. E. Osterhaus. 1994. Impairment of immune function in harbour seals (Phoca vitulina) feeding on fish from polluted waters. Ambio 23:155-159.

Doi, T. 1978. Discussion on body weight of baleen whales. Geiken tuushin (Whale Research Institute Newsletter) 318:57-64 (in Japanese). As cited in Ichii and Kato (1991).

Frisch, R. 1978. Population, food intake, and fertility. Science 199:22-30.

Frisch, R. 1990. The right weight: body fat, menarche and ovulation. Bailliere's clinical obstetrics and gynaecology 4:419-439.

Frisch, R., D. Hegsted, and K. Yoshinaga. 1975. Body weight and food intake 
at early estrus of rats on a high-fat diet. Proceedings of the National Academy of Science of the United States of America 72:4172-4176.

Fujiwara, M., and H. Caswell 2001. Demography of the endangered North Atlantic right whale. Nature 414:537-541.

Gopalan, C., and A. Naidu. 1972. Nutrition and fertility. Lancet 2:1077-1079.

Greene, C., A. Pershing, R. Kenney, and J. Jossi. 2003. Impact of climate variability on the recovery of endangered North Atlantic right whales. Oceanography 16:98-103.

Guo, Y., G. Lambert, C. C. Hsu, and M. M. Hsu. 2004. Yucheng: health effects of prenatal exposure to polychlorinated biphenyls and dibenzofurans. International Archives of Occupational and Environmental Health 77:153-158.

Gurney, W. S. C., and R. M. Nisbet. 1998. Ecological Dynamics. Oxford University Press, NY. ISBN 0-19-510443-9.

Gurney, W. S. C., and R. M. Nisbet. 2004. Resource allocation, hyperphagia and compensatory growth. Bulletin of Mathematical Biology 66:1731-1753.

Gurney, W. S. C., E. McCauley, R. M. Nisbet, and W. W. Murdoch. 1990. The physiological ecology of daphnia : A dynamic model of growth and reproduction. Ecology 71:716-732.

Hallam, T. G., R. R. Lassiter, J. Li, and L. A. Suarez. 1990. Modelling individuals employing an integrated energy response: Application to daphnia. Ecology 71:938954.

Hamilton, P., M. Marx, and S. Kraus. 1995. Weaning in north Atlantic right whales. Marine Mammal Science 11:386 - 390.

Hickie, B., D. Mackay, and D. Muir. 1997. Use of novel pharmacokinetic models to estimate contaminant elimination rates in marine mammals. 18th Annual Meeting of the Society of Environmental Toxicology and Chemistry, San Francisco, CA. SETAC Press, Pensacola, Florida.

Hickie, B. E., D. Mackay, and J. De Koning. 1999. Lifetime pharmacokinetic 
model for hydrophobic contaminants in marine mammals. Environmental Toxicology and Chemistry 18:2622-2633.

Hickie, B. E., M. C. S. Kingsley, P. V. Hodson, D. C. G. Muir, P. Beland, and D. Mackay. 2000. A modelling-based perspective on the past, present, and future polychlorinated biphenyl contamination of the st. Lawrence 800 beluga whale (Delphinapterus leucas) population. Canadian Journal of Fisheries and Aquatic Sciences suppl. 1, 57:101-112.

Ichii, T., and H. Kato. 1991. Food and daily food-consumption of southern minke whales in the Antarctic. Polar Biology 11:479-487.

Innes, S., D. M. Lavigne, W. M. Earle, and K. M. Kovacs. 1987. Feeding rates of seals and whales. Journal of Animal Ecology 56:115-130.

Iverson, S. 2002. Blubber. In Perrin, W. F. and Wuersig, B. G. and Thewissen, J. G. M., editors. Encyclopedia of marine mammals. Academic Press, San Diego, CA. ISBN: 0125513402.

Jørgensen, E., B. Bye, and M. Jobling. 1999. Influence of nutritional status on biomarker responses to $\mathrm{PCB}$ in the arctic charr (Salvelinus alpinus). Aquatic Toxicology 44:233-244,

Kann, L. M., and K. Wishner. 1995. Spatial and temporal patterns of zooplankton on baleen whale feeding grounds in the southern gulf of Maine. Journal of Plankton Research 17:235-262.

Kenney, R. D., C. A. Mayo, and H. E. Winn. 2001. Migration and foraging strategies at varying spatial scales in western North Atlantic right whales: a review of hypotheses. Journal of Cetacean Research and Management, Special Issue 2:251260.

Klanjscek, T., H. Caswell, M. G. Neubert, and R. M. Nisbet 2006. Integrating dynamic energy budgets into matrix population models. Ecological Modelling, in Press.

Kleiber, M. 1961. The fire of life. Wiley and Sons Inc., New York and London. 
Knowlton, A. R., S. D. Kraus, and R. D. Kenney. 1994. Reproduction in NorthAtlantic right whales (Eubalaena glacialis). Canadian Journal of Zoology 72:12971305.

Kooijman, S. A. L. M. 2000. Dynamic energy and mass budgets in biological systems, 2nd edition. Cambridge university press, Cambridge, UK. ISBN 052178608 8.

Koopman, H. N., D. A. Pabst, W. A. McLellan, R. M. Dillaman, and A. J. Read. 2002. Changes in blubber distribution and morphology associated with starvation in the harbor porpoise (Phocoena phocoena): evidence for regional differences in blubber structure and function. Physiological and Biochemical Zoology 75:498 - 512.

Kraus, S. D., P. K. Hamilton, R. D. Kenney, A. R. Knowlton, and C. K. Slay. 2001. Reproductive parameters of the North Atlantic right whale. Journal of Cetacean Research and Management, Special Issue 2:231—236.

Lee, J. S., S. Tanabe, H. Umino, R. Tatsukawa, and D. C. Loughlin, T. R. Calkins. 1996. Persistent organochlorines in Steller sea lion (Eumetopias jubatus) from the bulk of Alaska and the Bering Sea, 1976-1981. Marine Pollution Bulletin 32:535-544.

Lee, P. 1987. Nutrition, fertility and maternal investment in primates. Journal of Zoology (London) 213:409-422.

Leung, H., D. Paustenbach, F. Murray, and M. Andersen. 1990a. A physiological pharmacokinetic description of the tissue distribution and enzyme-inducing properties of 2,3,7,8-tetrachlorodibenzo-p-dioxin in the rat. Toxicology and Applied Pharmacology 103:399-410.

Leung, H., A. Poland, D. Paustenbach, F. Murray, and M. Andersen. 1990b. Pharmacokinetics of [125i]-2-iodo-3,7,8-trichlorodibenzo-p-dioxin in mice: analysis with a physiological modeling approach. Toxicology and Applied Pharmacology 103:411419 .

Lika, K., and R. M. Nisbet. 2000. A dynamic energy budget model based on partitioning of net production. Journal of Mathematical Biology 41:361-386. 
Lockyer, C. 1976. Body weights of some species of large whales. Journal du Conseil International pour l'Exploration de la Mer 36:259-273.

Lockyer, C. 1981. Estimation of the energy costs of growth, maintenance and reproduction in the female minke whale, (Balaenoptera acutorostrata), from the southern hemisphere. Report of the International Whaling Commission 31:337-343.

Lockyer, C. 1986. Body fat condition in northeast Atlantic fin whales, Balaenoptera physalus, and its relationship with reproduction and food resource. Canadian Journal of Fisheries and Aquatic Sciences 43:142-147.

Martin, R. D., and A. M. MacLarnon. 1985. Gestation period, neonatal size and maternal investment in placental mammals. Nature 313:220 - 223.

Martineau, D., K. Lemberger, A. Dallaire, P. Labelle, T. P. Lipscomb, P. Michel, and I. Mikaelian. 2002. Cancer in wildlife, a case study: beluga from the St. Lawrence estuary, Quebec, Canada. Environmental Health Perspectives 110:285 - 292.

Mayo, C. A., and M. K. Marx. 1990. Surface foraging behaviour of the North Atlantic right whale, Eubalaena glacialis, and associated zooplankton characteristics. Canadian Journal of Zoology 68:2214 - 2220.

McCauley, E., W. Murdoch, R. Nisbet, and W. Gurney. 1990. The physiological ecology of daphnia I: Development of a model of growth and reproduction. Ecology 71:703-715.

Moore, J., A. Knowlton, S. Kraus, W. McLellan, and R. Bonde. 2005. Morphometry, gross morphology and available histopathology in North Atlantic right whale (Eubalaena glacialis) mortalities (1980-2002). Journal of Cetacean Research and Management, in press.

Muller, E. B., and R. M. Nisbet. 1997. Modeling the effect of toxicants on the parameters of dynamic energy budget models, in ASTM Special Technical Publication. ASTM, Conshohocken, USA.

Nisbet, R. M., S. Diehl, W. G. Wilson, S. D. Cooper, D. D. Donalson, and K. Kratz. 1997. Primary-productivity gradients and short-term population dynamics in 
open systems. Ecological Monographs 67:535 - 553.

Nisbet, R. M., E. B. Muller, K. Lika, and S. A. L. M. Kooijman. 2000. From molecules to ecosystems through dynamic energy budget models. Journal of Animal Ecology 69:913-926.

Noonburg, E. G., R. M. Nisbet, E. McCauley, W. S. C. Gurney, W. W. Murdoch, and A. M. De Roos. 1998. Experimental testing of dynamic energy budget models. Functional Ecology 12:211-222.

Nordoy, E. S., and A. S. Blix. 1985. Energy sources in fasting grey seal pups evaluated with computed tomography. American Journal of Physiology - Regulatory, Integrative and Comparative Physiology 249:471—476.

Nowacek, D., M. Johnson, and P. Tyack. 2004. North Atlantic right whales (Eubalaena glacialis) ignore ships but respond to alerting stimuli. Proceedings of the Royal Society of London, Series B: Biological Sciences 271:227—231.

Phillips, D.L., Smith, A.B., Burse, V.W., Steele, G.K., Needham, L.L. and Hannon, W.H. 1989. Half-life of polychlorinated biphenyls in occupationally exposed workers. Archives of environmental health 44:351-354.

Pond, C.M. 1987. Some conceptual and comparative aspects of body composition analysis. In Toates, F.M. and Rowland, N. (eds): Methods and techniques to study feeding and drinking behaviour (pp. 499-529). Elsevier, Amsterdam.

Porter, W., J. Sabo, C. Tracy, O. Reichman, and N. Ramankutty. 2002. Physiology on a landscape scale: Plant-animal interactions. Integrative and Comparative Biology 42:431-453.

Porter, W. P., S. Budaraju, W. E. Stewart, and N. Ramankutty. 2000. Calculating climate effects on birds and mammals: Impacts on biodiversity, conservation, population parameters, and global community structure. American Zoologist 40:597630.

Reeves, R. R., J. M. Breiwick, and E. Mitchell. 1992. Pre-exploitation abundance of right whales off the eastern United States. In: Hain, J., editor: Right whales in the 
western North Atlantic: a management workshop. N.E. Fisheries Center Document 92:5-7.

Reijnders, P. J. H. 1986. Reproductive failure in common seals feeding on fish from polluted coastal waters. Nature 324:456 - 457 .

Reilly, J. J. 1991. Adaptations to prolonged fasting in free-living weaned gray seal pups. American Journal of Physiology. Regulatory, Integrative and Comparative Physiology 260:267-272.

Reilly, S., S. Hedley, J. Borberg, R. Hewitt, D. Thiele, J. Watkins, and M. Naganobu. 2004. Biomass and energy transfer to baleen whales in the south Atlantic sector of the southern ocean. Deep Sea Research II 51:1397-1409.

Restum, J., S. Bursian, J. Giesy, J. Render, W. Helferich, E. Shipp, D. Verbrugge, and R. Aulerich. 1998. Multigenerational study of the effects of consumption of PCB-contaminated carp from Saginaw bay, Lake Huron, on mink. 1. Effects on mink reproduction, kit growth and survival, and selected biological parameters. Journal of Toxicology and Environmental Health A 54:343-375.

Rosenbaum, H. C., R. L. Brownell, M.W. Brown, C. Schaeff, V. Portway, B.N. White, S. Malik, L.A. Pastene, N.J. Patenaude, C.S. Baker, M. Goto, P.B. Best, P.J.Clapham, P. Hamilton, M. Moore, R. Payne, V. Rowntree, C.T. Tynan, J.L. Bannister, and R. DeSalle. 2000. World-wide genetic differentiation of Eubalaena: questioning the number of right whale species. Molecular Ecology 9:1793-1802.

Ross, A. H., and R. M. Nisbet. 1990. Dynamic models of growth and reproduction of the mussel Mytilus edulis L. Functional Ecology 4:777-787.

Ross, P. S., R. L. De Swart, R. F. Addison, H. Van Loveren, J. Vos, and A. Osterhaus 1996. Contaminant-induced immunotoxicity in harbour seals: Wildlife at risk? Toxicology 112(2):157-169.

Ross, P. S., G. M. Ellis, M. G. Ikonomou, L. G. Barrett-Lennard, and R. F. Addison. 2000. High PCB concentrations in freeranging pacific killer whales, Orcinus orca: Effects of age, sex and dietary preference. Marine Pollution Bulletin 40:504- 
515.

Schwacke, L. H., E. O. Voit, L. J. Hansen, R. S. Wells, G. B. Mitchum, A. A. Hohn, and P. A. Fair. 2002. Probabilistic risk assessment of reproductive effects of polychlorinated biphenyls on bottlenose dolphins (Tursiops truncatus) from the southeast United States coast. Environmental Toxicology and Chemistry 21:27522764 .

Struntz, D. J., W. A. McLellan, R. M. Dillaman, J. E. Blum, J. R. Kucklick, and D. Pabst. 2004. Blubber development in bottlenose dolphins (Tursiops truncatus). Journal Morphology 259:7-20.

The North Atlantic Right Whale Recovery Team. 2000. Canadian Recovery Plan for the North Atlantic Right Whale. World Wildlife Fund Canada and the Department of Fisheries and Oceans.

Thomas, P. O., and S. M. Taber. 1984. Mother-infant interaction and behavioral development in southern right whales, Eubalaena australis. Behaviour 88:42 — 46 .

Thomas, P. T., and R. D. Hinsdill. 1980. Perinatal PCB exposure and its effect on the immune system of young rabbits. Drug and Chemical Toxicology 3:173-184.

Trites, A., and D. Pauly. 1998. Estimating mean body masses of marine mammals from maximum body lengths. Canadian Journal of Zoology 76:886 — 896.

Van der Spuy, Z. 1985. Nutrition and reproduction. Bailliere's Clinical Obstetrics and Gynaecology 12:579-604.

von Bertalanffy, L. 1957. Quantitative laws in metabolism and growth. Quarterly Review of Biology 32:217-231.

Weisbrod, A. V., D. Shea, M. J. Moore, and J. J. Stegeman. 2000. Organochlorine exposure and bioaccumulation in the endangered northwest Atlantic right whale (Eubalaena glacialis) population. Environmental Toxicology and Chemistry 19:654666.

Wells, R., V. Tornero, A. Borrell, A. Aguilar, T. Rowles, H. Rhinehart, S. Hofmann, W. Jarman, A. Hohn and J. Sweeney. 2005. Integrating life-history and re- 
productive success data to examine potential relationships with organochlorine compounds for bottlenose dolphins (Tursiops truncatus) in Sarasota Bay, Florida. Science of the Total Environment. 349:106-119.

Winn, H. E., C. A. Price, and P. W. Sorensen. 1986. The distributional biology of the right whale (Eubalaena glacialis) in the western North Atlantic. Report of the International Whaling Commission, Special Issue 10:129-138.

Wishner, K., E. Durbin, A. Durbin, M. Macaulay, H. Winn, and R. Kenney. 1988. Copepod patches and right whales in the great south channel off New England. Bulletin of Marine Science 43:825-844.

Young, R. 1976. Fat, energy and mammalian survival. American Zoologist 16:699-710. 


\subsection{Appendix}

\subsubsection{Morphometrics}

To parameterize the energetics part of the model, we first need to determine the relationship between the volume of structure and the length of the right whales. There are two independent data-sets with different physiological measurements. The first is the summary of 1970-2002 northern right whale (Eubalaena glacialis) necropsies by Moore et al (2005), which contains lengths, maximum girths, and blubber depths for four whales ranging from 4.16 to 13.6 meters in length. In the second one, Lockyer (1976) summarizes the body length, total mass, and blubber mass for the North Pacific right whale (Eubalaena glacialis sieboldii) for 13 whales ranging from 11.7 to $17.4 \mathrm{~m}$ in length.

In both cases, we start by relating the weights to volume through $\rho_{S}=1070 \mathrm{~kg} / \mathrm{m}^{3}$, the density of structure, and $\rho_{b}=900 \mathrm{~kg} / \mathrm{m}^{3}$, the density of blubber (Nordoy and Blix 1985). The mass $W$ is the sum of the volume of structure (proportional to length cubed) multiplied by its density and the volume of blubber multiplied by the blubber density:

$$
W=k l^{3} \rho_{S}+V_{b} \rho_{b},
$$

where $l$ is the length of the whale in meters.

To use data from Moore et al (2005), we rearrange (2.App.1) using $p_{V b}$, the ratio of the blubber and the total volume:

$$
W=k l^{3}\left(\rho_{S}+\frac{p_{V b}}{1-p_{V b}} \rho_{b}\right) .
$$

To estimate $p_{V b}$, we approximate the whale with a rotationally symmetric ellipsoid whose longer axis is $l / 2$, and minor axis is half the height, which is the maximum girth $l_{g}$, divided by $2 \pi$. Such a minor axis includes both the structure and the blubber, hence we subtract the thickness of the blubber, $l_{b}$, from the minor axis. Then, the 
volume of the blubber is the difference between the volumes of the ellipsoids described by the two minor axis. Rearranging gives $p_{V b}$ :

$$
p_{V b}=\frac{\left(l_{g}\right)^{2}-\left(l_{g}-2 \pi l_{b}\right)^{2}}{\left(l_{g}\right)^{2}} .
$$

Using $W, l, l_{g}$ and $l_{b}$ from Moore et al (2005),

$$
V=0.009 l^{3} .
$$

To use data from Lockyer (1976), we rearrange (2.App.1) using $p_{W b}$, the ratio of blubber and total mass:

$$
W=k l^{3} \rho_{S}+p_{W b} \rho_{b} .
$$

The ratio of blubber to total mass is easily calculated from the blubber mass and total mass given by Lockyer (1976). Moore et al (2005) had data for one right whale (MH89-424-Eg) with all the necessary data, so we added it to the data for 13 right whales in Lockyer (1976). The resulting relationship between the volume of the structure of a right whale and its length is:

$$
V=0.009 l^{3} .
$$

This is close to the relationship between mass of the whale and its length found by Doi (1978, as cited in Ichii and Kato 1991):

$$
W=0.011 l^{3} .
$$

The difference between the two values may come from the fact that we used just volume (proportional to mass) of the structure, whereas Doi (1978) used weights of whole whales. Note that the whale added from Moore et al (2005) to the Lockyer (1976) data set did not have blubber depths, and has, therefore, not been used in determining (2.App.4). Hence, the two data sets are completely independent and 
use different approaches to calculate the relationship between volume and length of a right whale. Note that this volume does not depend on the nutritional status of the whale, as it does not include its blubber.

\subsubsection{Energetics}

\section{Energy conductivity $\left(\beta_{L}\right)$ and rate of utilization of lipids for growth $\left(\beta_{G}\right)$}

Since our modeling framework is new, we did not find literature that uses the same definition of the rate of utilization of energy and the energy conductivity; hence, we were unable to get a good estimate of these parameters. Storage and utilization of lipids is a fairly fast process that has to keep up with daily changes in the balance of energy acquisition and utilization, while growth (and, therefore, utilization of energy for growth) is a much slower process. Hence, we estimate that the time-scale for $\beta_{L}$ should be days, and weeks for $\beta_{G}$. Thus, we (arbitrarily) set

$$
\beta_{L}=365 \mathrm{y}^{-1} \text { and } \beta_{G}=52 \mathrm{y}^{-1} \text {. }
$$

\section{Cost of maintenance per unit of volume $(m)$}

The energetic cost of maintenance $(m)$ can be roughly estimated from power-laws of adult maintenance energy requirements. We use the power laws to estimate the maintenance requirements for a typical adult whale $\left(65,000 \mathrm{~kg}, V_{\infty}=36.9 \mathrm{~m}^{3}\right)$. Since energy reserves do not need maintenance (Kooijman 2000), and we assume whales do not spend energy on thermoregulation, this estimate directly corresponds to $F_{B M}$ in our model. Hence, we can calculate $m$ using (2.1).

Kleiber (1961) and Brody (1968) compare metabolic rates for many species. They estimate that the resting metabolic rate of species whose adults typically weigh $W$ (in $\mathrm{kg}$ ) is

$$
F_{B M}^{\prime} \approx 70 W^{3 / 4}
$$


Note that (2.App.9) describes inter-specific variation, and is not inconsistent with equation (2.1). Lockyer (1976) modifies (2.App.9) to include foraging,

$$
F_{B M} \approx 110 W^{0.783} \mathrm{kcal} / \text { day. }
$$

Averaging the data from Lockyer (1976) suggests that the average adult right whale is $16 \mathrm{~m}$ long, implying a volume of $36.9 \mathrm{~m}^{3}$ and weighs about $65,000 \mathrm{~kg}$. According to (2.App.10), such a whale requires $F_{B M}$ of about $645,500 \mathrm{kcal} /$ day, so we can calculate $m$ using $(2.1)$ :

$$
m=6.33 \cdot 10^{6} \mathrm{kcal} / \mathrm{m}^{3} \mathrm{y} .
$$

\section{Cost of growth by a unit of volume $(g)$}

We calculate the energetic cost of growth $(g)$ by considering the estimates for energetic costs of growth of marine mammals, which range from $1500 \mathrm{kcal} / \mathrm{kg}$ for minke whales (Lockyer 1981) to $9100 \mathrm{kcal} / \mathrm{kg}$ for dolphins (Hickie et al. 2000). Methods used to measure and estimate the costs of growth in these studies do not directly correspond to $g$ because they either include only the cost of muscle growth, or the cost of increasing lipid energy storage, neither of which corresponds to our definition of growth. Therefore, we adopt an intermediate value for $g$, the estimate for phocid seals of $4147 \mathrm{kcal} / \mathrm{kg}$ (Innes et al. 1987). Multiplying the value with the density of structure $\rho_{s}=1070 \mathrm{~kg} / \mathrm{m}^{3}$ (Nordoy and Blix 1985), gives the volume-specific cost of growth:

$$
g=(4147 \mathrm{kcal} / \mathrm{kg}) 1070 \mathrm{~kg} / \mathrm{m}^{3} \approx 4.4 \cdot 10^{6} \mathrm{kcal} / \mathrm{m}^{3} .
$$

The estimates of $m$ and $g$ reflect the assumption that lipids alone fuel maintenance and growth. Whales, however, ingest and use other forms of energy in addition to lipids. Although the other forms of energy do not influence lipid transport and storage 
because we assume the proportions of all forms of energy are constant, we lack the data to account for other energy sources in parameter estimation. Therefore, we may be overestimating energy that is needed in the form of lipids.

\section{Maximum assimilation rate per unit area of the whale $\left(I_{\max }\right)$}

From $V_{\max }$, we calculate $I_{\max }$ using the maximum observed size of a right whale as a conservative estimate of the maximum size.

The maximum observed right whale length $l_{\max }=18 \mathrm{~m}$ (Trites and Pauly 1998) is a conservative estimate of the maximum possible length. Combining (2.7) and (2.App.6) gives a conservative estimate of the maximum assimilation rate per unit area:

$$
I_{\max }=m 0.208 l_{\max }=2.41 \cdot 10^{7} \mathrm{kcal} \mathrm{m}^{-2} \mathrm{y}^{-1} .
$$

Since it is unlikely that any right whale in nature experienced complete saturation in food throughout their lifetime, our estimate of $I_{\max }$ is most likely an underestimate. The resulting energy assimilation rate for an average-sized right whale of approximately $2.7 \cdot 10^{8} \mathrm{kcal} / \mathrm{y}$ is consistent with the estimated energy ingestion rate for right whales of $2 \cdot 10^{8}-6 \cdot 10^{8} \mathrm{kcal} / \mathrm{y}$ (Reilly et al. 2004), especially since the estimated ingestion rate does not take energy assimilation efficiency into the account. However, our value is on the lower end of the Reilly et al. (2004) estimate, suggesting that we have indeed underestimated $I_{\max }$, possibly by $50 \%$ or more. This does not have serious consequences on our analyses because the organism responds to $F_{I B}$, which is also a function of $f$, rather than $I_{\max }$.

\section{Equilibrium ratio constant $\left(k_{L}\right)$}

We first assume a whale that is not growing, in a constant environment. This implies that $E_{B}$ and $E_{L}$ are in equilibrium. We can calculate the equilibrium $E_{B}$ from $\beta_{G}$, and estimate $E_{L}$ from observations. The equilibrium coefficient then follows from the ratio of $E_{B}$ and $E_{L}$. 
First, we calculate $\overline{E_{L}}$, the typical energy in the lipid storage compartment $\left(E_{L}\right)$ for an adult whale that is neither reproducing nor growing. The blubber (majority of lipid energy storage) constitutes about $21 \%$ of the whale mass using data from Moore et al (2005), and about 40\% using Lockyer (1976) data for adults. Although different, these values are consistent because Moore et al (2005) use post-mortem analyses of often emaciated whales, whereas Lockyer (1976) uses right whale whaling data, where the capture process favors individuals with more blubber. Using $40 \%$ as a value that is closer to those typically found in the wild, and a caloric content of the blubber of $9320 \mathrm{kcal} / \mathrm{kg}$ (Nordoy and Blix), we estimate $\overline{E_{L}}$ :

$$
\overline{E_{L}}=65000 \mathrm{~kg} 9320 \mathrm{kcal} / \mathrm{kg} 0.4=242.32 \cdot 10^{6} \mathrm{kcal} .
$$

Next, we estimate $\overline{E_{B}}$, the typical energy in the blood compartment $\left(E_{B}\right)$ for an adult whale that is neither reproducing nor growing. For such an adult, $F_{B G}=0$ and $F_{B L}=F_{L B}$. Using $(2.2)$, we calculate $\overline{E_{B}}$ :

$$
\overline{E_{B}}=\frac{F_{B M}}{\beta_{G}} .
$$

Finally, from $(2.8) k_{L}$ is the ratio between $\overline{E_{B}}$ and $\overline{E_{L}}$ :

$$
k_{L}=0.02
$$

\section{Duration of gestation and lactation $\left(\tau_{\text {gestation }}\right.$ and $\left.\tau_{\text {lactation }}\right)$}

Gestation in right whales lasts about a year (Best 1994). We assume that lactation lasts one year as well, although both longer (Thomas and Taber 1984) and shorter (Hamilton et al. 1995) times have been observed. 


\section{Minimum energy required for reproduction $\left(E_{R}\right)$}

We use observations of blubber thickness from Angell (2005) and our ellipsoid analogue of the whale to estimate the minimum energy required for reproduction, $E_{R}$. During the reproductive season, reproduction starts if $E_{L} \geq E_{R}$. Angell (2005) note that the blubber thickness of the mother reduces by about $4.5 \mathrm{~cm}$ during a reproductive event. The female whale NEAq1223 died very close to weaning of her calf. Her blubber depth was $16.2 \mathrm{~cm}$, which implies that her blubber depth at conception was $21.7 \mathrm{~cm}$. Using the ellipsoid analogue of the whale, her length $(13.6 \mathrm{~m})$, and girth $(8.8 \mathrm{~m})$, we calculate the volume of blubber female NEAq1223 had at conception to be $16.65 \mathrm{~m}^{3}$. Using the density of the blubber of $900 \mathrm{~kg} / \mathrm{m}^{3}$ and its caloric content of $9320 \mathrm{kcal} / \mathrm{kg}$ (Nordoy and Blix 1985), we can estimate the energy needed to conceive:

$$
E_{R}=1.4 \cdot 10^{8} \mathrm{kcal} .
$$

\section{Gestation (a)}

We calculate the constant $a$ from the known size of the neonate. Taking a typical length of a neonate of $480 \mathrm{~cm}$ from Moore et al (2005), and using (2.App.6) the typical volume of a neonate is $V_{N} \approx 1 \mathrm{~m}^{3}$. From (2.13),

$$
\begin{aligned}
a & =1.25 \frac{V_{N}}{\tau_{\text {gestation }}} \\
& =1.25 \mathrm{~m}^{3} / \mathrm{y} .
\end{aligned}
$$

\section{Lactation $\left(\beta_{v B}\right.$ and $\left.l_{\max }\right)$}

To estimate the energy the calf demands we first need to estimate its ideal growth curve. We do this by considering how much energy it would need to grow following a von Bertalanffy growth curve starting from the neonatal length $l_{N}=4.8 \mathrm{~m}$ and trying to reach the maximum length observed for the species $\left(l_{\max }=18 \mathrm{~m}\right)$. 
The length of an organism growing following the von Bertalanffy growth curve is:

$$
l_{v B}(t)=l_{\max }-\left(l_{\max }-l_{N}\right) e^{-\beta_{v B} t} .
$$

To evaluate (2.App.20), we need $\beta_{v B}$. Solving (2.App.20) for $\beta_{v B}$ :

$$
\beta_{v B}=-\frac{1}{t} \ln \frac{l_{\max }-l(t)}{l_{\max }-l_{N}}
$$

Using data from Table 4 in Moore et al (2005) gives

$$
\beta_{v B}=0.35
$$

Note that the calculated $\beta_{v B}$ is a result of a fit of the von Bertalanffy growth curve to data from Moore et al (2005), but the growth the calves are aspiring to $\left(l_{\max }\right)$ is $18 \mathrm{~m}$, not $15 \mathrm{~m}$ observed by Moore et al (2005) and used in the calculation of $\beta_{v B}$. The shorter observed terminal length is a result of environmental conditions experienced, which may be far from the ideal that a calf demands. We assumed the data from Moore et al (2005) describe ideal conditions when calculating the von Bertalanffy growth rate constant $\beta_{v B}$ describing early growth, but not when calculating the asymptotic length of the calf, $f_{v B}(t)$. The estimate is appropriate for our purpose because we use the formula to put an upper bound on energy requirement of the calf during lactation, and only use the initial part of the growth curve. The real flux of energy from the mother is constrained by the mother's own energy budget.

\section{Reproductive efficiency $\left(k_{R}\right)$}

Neither gestation, nor lactation are completely efficient. Hickie et al. (2000), uses the reproductive efficiency coefficient during gestation of 0.7, and Lockyer (1981) estimates the combined efficiency of milk production and consumption of 0.72. Since these efficiencies are similar, we use an efficiency $k_{R}=0.7$ for both. 


\subsubsection{Pharmacokinetics $\left(D_{L S}\right.$ and $\left.C_{I}\right)$}

To include diffusion into the model, we need to estimate $D_{L S}$. Aguilar and Borrell (1991) determined concentrations of PCBs and DDTs in inner and outer strata of baleen (fin and sei) whales. These strata correspond to structural lipid and lipid storage compartments in our model.

Aguilar and Borrell (1991) show that the organochlorine levels in the structural lipids are significantly higher than those in the energy storage lipids. Also, they show that male whales have higher concentrations of toxicants in both types of lipids and contribute the observed difference between sexes to the losses of toxicants during reproduction.

The observation that both types of lipids differed between sexes suggests that there is a mechanism (diffusion in our model) that equilibrates concentrations between structural and energy storage lipids. If there were no diffusion, females would have similar outer but lower inner layer concentrations compared to males because the structural lipids are, for the most part, formed before females mature sexually and would, therefore, have undergone the same dynamics as that of a male. The observation that the two types of lipids have significantly different concentrations of toxicants within each gender means that the equilibration exists.

Equilibration may be slow, however. The whales examined by Aguilar and Borrell (1991) were captured during commercial hunt at the height of the whale feeding season. The newly acquired energy stores reduced concentrations of organochlorines in the deep blubber. Since the outer, metabolically inactive layers, had higher concentrations, presumably experienced by the energy storage lipids during the starvation season, we conclude that the two concentrations equilibrate on a time-scale longer than 0.5 years. On the other hand, the variability of concentrations in structural blubber among females was low, which suggests that the structural blubber equilibrated with the changes experienced between two reproductive events (when the toxicant loss occurs). This, in turn, suggests that the diffusion operates on time- 
scales shorter than 1.5 years.

Combining the two gives an estimate for the time-scale of the diffusion $\left(D_{L S} V^{2 / 3}\right)$ of one year. Since all captured whales were adults, we can estimate $D_{L S}$ using the typical volume $V_{\infty}$ of an adult whale:

$$
D_{L S}=0.09 \mathrm{~m}^{-2} \mathrm{y}^{-1} .
$$

Food is the primary vector of toxicant introduction in marine mammals. Right whales feed primarily on zooplankton, such as krill and various species of Calanus (Kann and Wishner 1995, Baumgartner and Mate 2003). There are many toxicants we could focus on. Their concentrations and, consequently, intake from the environment depend on the location and the species of zooplankton right whales feed on. For simplicity, we focus on polychlorinated biphenyls (PCBs), neglect all differences between different congeners of PCBs, and assume a constant total PCB concentration in the prey of approximately $0.035 \mathrm{mg} \mathrm{PCB} / \mathrm{kg}$ lipid, as measured in Calanus glacialis (Borga and Di Guardo 2005). Weisbrod et al. (2000) did not observe significant differences between total PCBs in the prey and in the fecal matter, suggesting that right whales do not seem to preferentially retain PCBs (Weisbrod et al. 2000). This implies that the input concentration $C_{I}$ is equal to the environmental concentration $\left(C_{I}=0.035 \mathrm{mg} / \mathrm{kg}\right)$. 


\section{Chapter 3}

\section{Bioaccumulation and effects of exposure in marine mammal populations}

We used to think that if we knew one, we knew two, because one and one are two. We are finding that we must learn a great deal more about 'and'. (Sir Arthur Eddington, astronomer)

\subsection{Introduction}

Food is necessary for growth and survival, but can have negative consequences if it contains toxicants. Effects of toxicants can be short-term (acute) or long-term (chronic) and generally increase with the amount of toxicants acquired. Directly or indirectly, effects of exposure to toxicants reduce survival probability and/or reproductive ability of individuals over their lifetime. This in turn reduces population growth rate. 
Lipophilic persistent toxicants such as polychlorinated biphenyls (PCBs) can bioaccumulate, increasing in concentration with time and creating potential for greater harm (Aguilar and Borrell, 1994, Borga et al. 2004). Large amounts of energy transferred from marine mammal mothers to their young during gestation and nursing facilitate transfer of toxicants between generations. Food scarcity increases the transfer because mothers bioaccumulate more between reproductive events (see Chapter 2).

The transfer of toxicants is good for the mother because it reduces her burden, but bad for the young because it exposes them to toxicant concentrations an order of magnitude higher than environmental (Lee et al. 1996). The exposure occurs at a critical time in young mammal development and could reduce their life span and reproductive ability (Reijnders 1986, de Swart et al. 1994, Lai et al. 2001, Schwacke et al. 2002).

Maternal transfer could have a strong influence on toxicant exposure of marine mammals and pose a risk to marine mammal populations in addition to the risk from environmental exposure. Without transfer, mothers may accumulate greater toxicant burdens and experience greater reduction in survival and reproduction as a result. On the other hand, if there is maternal transfer, toxicant concentrations in reproducing females are reduced but young are exposed to toxicants earlier, leading to higher toxicant levels in males and immature females. Which of these two cases has a greater effect on the population growth rate is unknown. In general, the effects of food availability, maternal transfer and effects of exposure on patterns of bioaccumulation and the population growth rate are largely unknown.

The lack of knowledge about the effects of toxicants on marine mammal populations is partly due to difficulties in experimenting on marine mammals. Compared to the usual animal models in toxicology (mice, rats and mink), marine mammals are large, long-lived, have small populations, and are difficult to experiment on because of space, moral and regulatory restrictions. Because of their large size, samples rep- 
resent a small fraction of body tissue, which complicates measurements because of heterogeneities within tissues (Aguilar and Borrell 1991). Longevity makes it hard to investigate long-term consequences of exposure, both on individuals and on populations. Small population sizes make it hard to accumulate enough data to obtain statistical significance. Whereas the other restrictions (space, legalities, ethics and regulations) may not present a significant obstacle in experimenting on terrestrial mammals, especially rodents, they do make it difficult to experiment on marine mammals (but see Restum et al. 1998, Ross et al. 1996, Wells et al. 2005). Consequently, experiments are rare, costly, and often executed with very low numbers of individuals. Observing animals in the wild avoids some of the above mentioned problems, but the data are often incomplete, and many possible causes (exposure to mixtures of substances, environmental variability, harvesting, predation, disease, etc.) for a particular effect requires a multivariate approach and, consequently, a large quantity of data that are hard to obtain.

Theoretical models can help overcome some of these difficulties. They can guide experimental research and reduce the necessary amounts of data. Two types of models are often used for this purpose: pharmacokinetic models (Boon et al. 1994, Hickie et al. 1999, Emond et al. 2005) that determine distribution of the toxicant throughout the body, and energy budget models that determine energy intake and utilization and, because energy and toxicants are connected, help drive the pharmacokinetic models (Kooijman and van Haren 1990, Muller and Nisbet 1997, Hickie et al. 2000, Chapter 2). Models of toxicant action connect the distribution of the toxicant to the effects on individuals.

Energy budget models come in two flavors: demand-side, where the energy intake and its utilization is determined exclusively from the needs of the individual organism, and supply-side models where both food availability and the needs of the organism control energy intake.

To investigate the dependence of food availability and bioaccumulation patterns of 
an individual, one needs a supply-side energy budget model coupled with a pharmacokinetic model. I presented such a model tailored to marine mammals in Chapter 2. Here I present a population model capable of describing the processes of energy and toxicant accumulation and utilization as functions of energy availability and environmental toxicant concentrations. The population model is an individual-based model (IBM) in which each individual grows according to the dynamic energy budget model of a marine mammal presented in Chapter 2, adapted for the right whale, Eubalaena spp. Using the model, I investigate asymptotic (long term) as well as transient (short term) effects of food availability, maternal transfer, and effects of exposure on bioaccumulation patterns and population growth rate.

I first present a brief description of the individual model for growth and reproduction (Section 3.2.1), then introduce mortality to complete the IBM (Section 3.2.2) and define effects of exposure in Section 3.2.2. In Section 3.3, I investigate the effects of exposure on bioaccumulation and population growth rate, and investigate how maternal transfer affects both. I discuss the results in Section 3.4.

\subsection{Methods}

\subsubsection{Individual energy budget and pharmacokinetic model}

The details of the model are explained in Chapter 2. Here I use the same basic structure of the model, and the same parameters for the right whale (Table 3.4), but a somewhat simplified model. The results of Chapter 2 suggest that structural lipids, and biotransformation of toxicants do not play a significant role for the parameter values corresponding to the right whale. This allowed me to simplify the model by ignoring toxicant biotransformation and the structural lipids.

For lipophilic toxicants, i.e. those that preferentially associate with lipids, the model of energy fluxes determines the toxicant fluxes in the organism. Figure 3-1 shows the structure of the simplified model: energy and toxicants enter from the 
environment (I) into the blood (B). From the blood, energy can be either stored into lipid energy storage $(\mathrm{L})$, or utilized for maintenance $(\mathrm{M})$, reproduction $(\mathrm{R})$ or growth (G). The compartments and their state variables are listed in Table 3.1.

Maintenance takes precedence over any other energy expenditure: if it is not met, the organism dies. The fluxes of energy from the environment into the blood $\left(F_{B G}\right)$, from the lipid energy storage into the blood $\left(F_{L B}\right)$, from the blood into the lipid energy storage $\left(F_{B L}\right)$, and from the blood to maintenance $\left(F_{B M}\right)$, reproduction $\left(F_{B R}\right)$ and growth $\left(F_{B G}\right)$ determine the rate of change of energy in the blood compartment (Table 3.2). Similarly, $F_{B L}$ and $F_{L B}$ determine the rate of change of energy in the lipid energy storage compartment. Fluxes are defined in Table 3.3; for a detailed description of reproduction see Chapter 2.

The transport of toxicants is linked to the transport of energy: when energy moves from one compartment to another, it also transfers toxicants in proportion to the concentration in the first compartment. Because I do not incorporate structural lipids in the simpler version of the model, there are no diffusion terms (see Chapter 2 for discussion), and the transport of toxicants completely depends on the fluxes of energy (Table 3.2).

The energy flux to reproduction is the same as in Chapter 2: energy transferred from the mother to her calf is a result of tug-of-war between the desire of the calf to grow, and the abilities of the mother to meet this desire. In Chapter 2, the energy transfer to the calf determined the toxicant transfer as well. As a consequence of losses in energy transfer (e.g. due to the cost of placenta and calf's assimilation inefficiency), approximately $30 \%$ of toxicants lost by the mother are not assimilated by the calf. The transfer can be further diminished by potential barriers to toxicant transfer. The barriers can exist both during gestation (e.g. the placenta) and during lactation (e.g. mammary glands, calf's digestive tract). Here I consider only barriers during lactation. In this chapter, I manipulate the toxicant transfer to account for the effects of these potential barriers. 


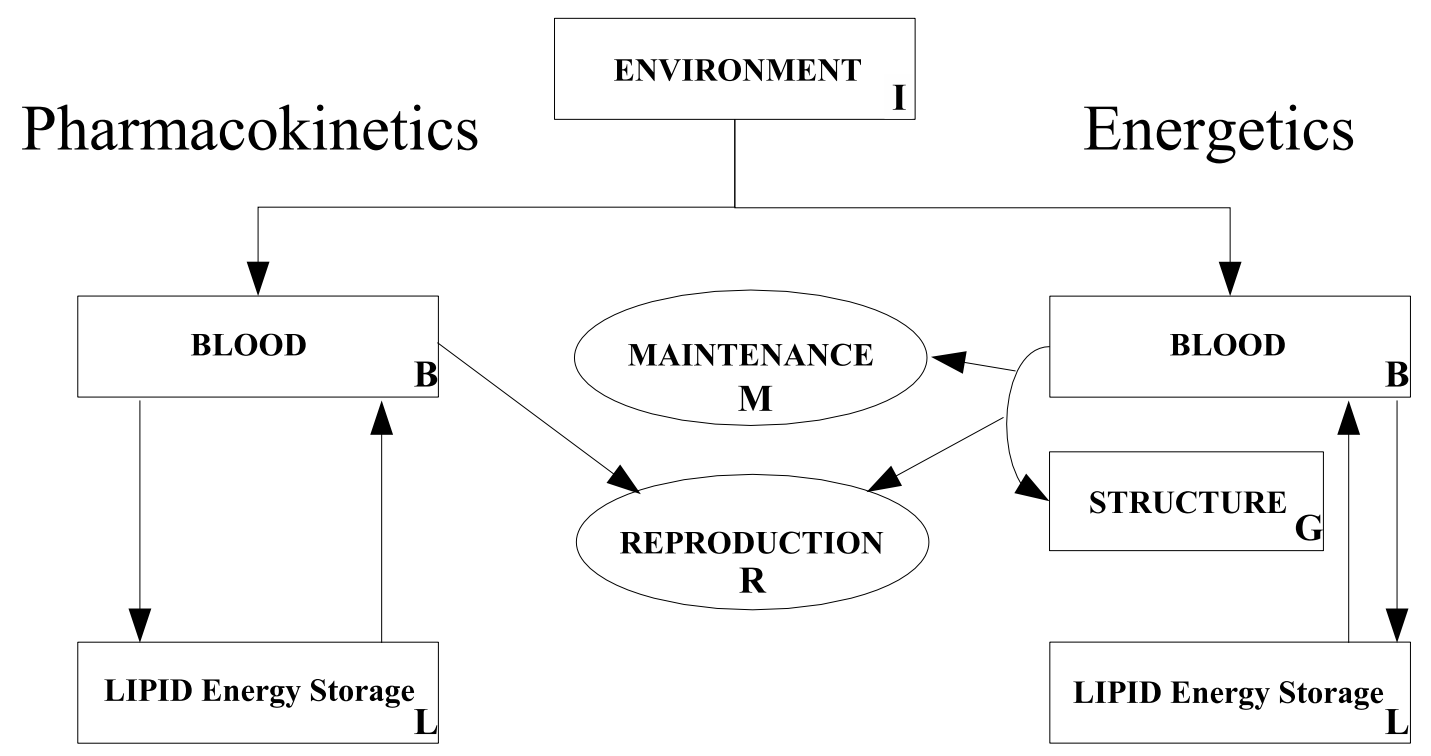

Figure 3-1: Individual model outline with pharmacokinetic (left) and energetic (right) model compartments. For simplicity, we ignore toxicant biotransformation.

If the barriers have no effect, mothers lose the toxicants, and calves assimilate them. I denote this mode of maternal transfer as $\mathrm{M}-\mathrm{C}+$, where $\mathrm{M}$ stands for mothers, $\mathrm{C}$ for calves, and the quantifier behind the letter denotes the dynamics of the toxicant due to maternal transfer: ' - ' for loss, ' + ' for gain, and ' 0 ' for no effect. If the barriers allow the mother to lose the toxicant, but prevent the calf from assimilating it, mothers lose and calves do not assimilate the toxicant ( $\mathrm{M}-\mathrm{C} 0$ mode). If the barriers are impenetrable to the toxicant, mothers do not lose, and calves do not assimilate the toxicant (M0C0 mode). Any other possible retention and assimilation mode is a combination of these three basic ones, and any of its effects should be within the range of effects of these three basic possibilities. 
Table 3.1: Compartments and state variables with units.

\begin{tabular}{llcc}
\hline \hline & \multicolumn{1}{c}{ Compartment } & Energetics & Toxicology $^{1}$ \\
\hline$I$ & Environment & $f$ & $C_{I}[\mathrm{mg} / \mathrm{kg}]$ \\
$G$ & Structure & $V\left[\mathrm{~m}^{3}\right]$ & - \\
$B$ & Blood & $E_{B}[\mathrm{kcal}]$ & $C_{B}[\mathrm{mg} / \mathrm{kg}]$ \\
$L$ & Lipid energy storage & $E_{L}[\mathrm{kcal}]$ & $C_{L}[\mathrm{mg} / \mathrm{kg}]$ \\
$R$ & Reproduction & - & - \\
$M$ & Maintenance & - & - \\
\hline \hline
\end{tabular}

${ }^{1}$ As in Chapter 2, our choices of units were guided by the literature A conversion factor connects fluxes of energy $([\mathrm{kcal} / \mathrm{y}])$ to fluxes of lipids $([\mathrm{kg} / \mathrm{y}])$. The factor has units of $\mathrm{kg}$ lipid per $\mathrm{kcal}$ $(\mathrm{kg} / \mathrm{kcal})$. We do not need to know its value, as it cancels out in the equations for rates of change of toxicant concentrations (Table 3.2).

Table 3.2: Kinetics: rates of change of state variables.

\begin{tabular}{cl}
\hline \hline Comp. & \multicolumn{1}{c}{ Dynamics $^{2}$} \\
\hline \multirow{2}{*}{$I$} & $\begin{array}{l}e_{I}=\text { function of } t \text { (constant in our simulations) } \\
\end{array}$ \\
\hline$G$ & $\frac{d}{d t} V=$ function of $t$ \\
\hline \multirow{2}{*}{$B$} & $\frac{d}{d t} E_{B}=F_{I B}+F_{L B}-F_{B L}-F_{B M}-F_{B G}-F_{B R}$ \\
& $\frac{d}{d t} C_{B}=\frac{1}{E_{B}}\left(C_{I} F_{I B}-C_{B}\left(F_{B L}+F_{B R}+\frac{d}{d t} E_{B}\right)+C_{L} F_{L B}\right)$ \\
\hline \multirow{2}{*}{$L$} & $\frac{d}{d t} E_{L}=F_{B L}-F_{L B}$ \\
& $\frac{d}{d t} C_{L}=\frac{1}{E_{L}}\left(C_{B} F_{B L}-C_{L}\left(F_{L B}+\frac{d}{d t} E_{L}\right)\right)$ \\
\hline \hline
\end{tabular}

Dynamics presented here differs from the one presented in Chapter 2 in three main details: in this chapter, $C_{I}$ may not be constant during simulations, there are no terms accounting for biotransformation of the toxicant, and there are no fluxes to or from the structural lipids compartment (as well as no dynamics for the structural lipids compartment).

Table 3.3: Equations for the energy fluxes.

\begin{tabular}{ll}
\hline \hline \multicolumn{1}{c}{ Flux [kcal/y] } & \multicolumn{1}{c}{ Description } \\
\hline$F_{I B}=I_{\max } f V^{2 / 3}$ & intake of energy from environment into blood \\
$F_{B M}=m V$ & energy spent on maintenance \\
$F_{B G}=\left[\beta_{G} E_{B}-F_{B M}\right]_{+}$ & energy utilized for growth ${ }^{3}$ \\
$F_{B L}=\beta_{L} E_{B}$ & energy flux from the blood to the lipid storage \\
$F_{L B}=\beta_{L} k_{L} E_{L}$ & energy flux from the lipid storage to the blood \\
$F_{B R}=\frac{1}{k_{R}}\left(F_{B R}^{M}+F_{B R}^{G}+F_{B R}^{E}\right)$ & flux of energy to reproduction (see Chapter 2) \\
\hline \hline
\end{tabular}

\footnotetext{
${ }^{1}[X]_{+}$is a shorthand notation for $\max (0, X)$.
} 
Table 3.4: Right whale parameter values.

\begin{tabular}{lcl}
\hline \hline Parameter & Value & \multicolumn{1}{c}{ Description } \\
\hline$\beta_{G}$ & $52 \mathrm{y}^{-1}$ & ENERGETICS \\
$\beta_{L}$ & $365 \mathrm{y}^{-1}$ & rate of utilization of lipids in blood \\
$m$ & $6.33 \cdot 10^{6} \mathrm{kcal} \cdot \mathrm{m}^{-3} \mathrm{y}^{-1}$ & energy conductivity \\
$g$ & $4.4 \cdot 10^{6} \mathrm{kcal} / \mathrm{m}^{-3}$ & energetic cost of growing structure \\
$k_{L}$ & 0.02 & equilibrium ratio constant of B and L \\
$I_{\max }$ & $2.41 \cdot 10^{7} \mathrm{kcal} \cdot \mathrm{m}^{-2} \mathrm{y}^{-1}$ & energy acquisition rate per biometric area \\
\hline \multicolumn{3}{c}{ REPRODUCTION } \\
\hline$E_{R \min }$ & $1.4 \cdot 10^{8} \mathrm{kcal}$ & minimum stored energy to start reproduction \\
$\tau_{\text {gestation }}$ & $1 \mathrm{y}$ & length of gestation \\
$\tau_{\text {lactation }}$ & $1 \mathrm{y}$ & length of lactation \\
$a$ & $1.25 \mathrm{~m}^{3} / \mathrm{y}$ & rate of growth during gestation \\
$\beta_{v B}$ & $0.35 \mathrm{y}^{-1}$ & von Bertalanffy rate constant \\
$V_{\max }$ & $52.5 \mathrm{~m}^{3}$ & maximum V under ideal conditions \\
$k_{R}$ & 0.7 & efficiency of reproduction \\
\hline \hline
\end{tabular}

\subsubsection{Modelling mortality}

To complete the population model, I need to account for mortality of individuals and the effects of exposure. Mortality can be modelled as a stochastic process that depends on $s$, the age of the individual (Cox and Oakes 1984, pp. 14): the probability of dying in the time it takes to grow older by $d s$, given that the individual is alive at age $s$, is equal to the product of $d s$ and $h(s)$, the age-dependent hazard rate. In my model, this holds independently for all weaned individuals. When a pregnant or nursing mother dies, her calf dies as well. If a calf dies, the mother simply stops her flux of energy to reproduction $\left(F_{B R}=0\right)$.

The hazard rate $(h(s))$ includes all contributions to mortality of an individual except deaths of calves resulting from the death of their mother. I separate $h(s)$ into three positive parts: $h_{I}(s)$, the intrinsic hazard rate due to damage accumulated through metabolic work and aging, $h_{C}$, the negative effects of hazardous compounds, 
and $h_{E}(s)$, external stressors such as gear entanglement and ship strikes:

$$
h(s)=h_{I}(s)+h_{C}+h_{E}(s) .
$$

If $h_{E}$ is a function of time, it may be expressed as a function of age as $s=t-t_{c}$, where $t_{c}$ is the time of conception. I assume $h_{E}(s)=0$ in this chapter.

From (3.1) and Kooijman (2000), the probability of having a life span $\tau$ greater than $s$ is

$$
\operatorname{Pr}\{\tau>s\}=\exp \left\{-\int_{0}^{s} h(\xi) d \xi\right\}
$$

The mean life span is

$$
\tau_{E}=\mathrm{E}[\tau]=\int_{0}^{\infty} \operatorname{Pr}\{\tau>s\} d s .
$$

\section{Intrinsic mortality}

I account for two distinct sources of intrinsic hazard: aging (aging hazard), and possible problems during early development (developmental hazard). I assume that the aging hazard increases linearly with age. The developmental hazard rate typically drops off exponentially with age, and is small by the time of weaning. The complete intrinsic hazard rate is then:

$$
h_{I}(s)=\eta_{0} \exp \left(-s / \tau_{\eta_{0}}\right)+\eta_{1} s
$$

where $\eta_{1} s$ is the aging, and $\eta_{0} \exp \left(-s / \tau_{\eta_{0}}\right)$ the developmental hazard. The exponential time constant of the developmental hazard rate, $\tau_{\eta_{0}}$, is $\tau_{\eta_{0}}=\left(\tau_{\text {gestation }}+\right.$ $\left.\tau_{\text {lactation }}\right) / 2=1 \mathrm{y}$.

I calculate $\eta_{0}$ and $\eta_{1}$ using the life expectancy of female right whales from Fujiwara and Caswell (2001). They found that the life expectancy of females declined from 50 years in 1980 to 20 years in 2000, and that the probability of the calf dying during the last six months before weaning is 0.08. Taking the life expectancy for 1988 of 25 
years and fitting (3.3) gives $\eta_{0}=0.09966 \mathrm{y}^{-1}$, and $\eta_{1}=0.00144 \mathrm{~kg} \cdot \mathrm{mg}^{-1} \mathrm{y}^{-1}$. Here I assumed that calf mortality is conditioned on mother's survival, and that the life expectancy of 25 years is a consequence of the intrinsic hazard only.

\section{Effects of exposure - mortality from toxicants}

Toxicants of ecological interest are those that affect survival and/or reproduction (Kooijman 2000). They can influence mortality and reproduction directly and indirectly by influencing parameters of the model. For example, an increase in $h_{C}$ directly increases mortality. Similarly, an increase in reproduction energy $\left(E_{R \min }\right)$ and a decrease in reproductive efficiency $\left(k_{R}\right)$ directly decrease reproduction. Toxicants can indirectly affect reproduction and mortality through effects on other model parameters (Muller and Nisbet 1997) such as foraging ability $(f)$, assimilation efficiency $\left(I_{\max }\right)$, maintenance costs $(m)$, and costs of growth $(g)$. In this chapter, I only implement direct effects of exposure on mortality through $h_{C}$, but effects on any and all parameters could be implemented using a similar approach.

Traditionally, direct effects on mortality are implemented through the hazard rate as a consequence of the concentration of toxicants in the organs of an individual (Kooijman 2000, pp. 204-206). I assume the concentration in the blood compartment, $C_{B}$, reflects the effective exposure of the organism, because it captures both shortterm (acute) exposure following an ingestion of the toxicant, as well as the long-term (chronic) exposure resulting from bioaccumulation. From the DEB theory (Kooijman 2000), the hazard rate from the accumulated toxicant is

$$
h_{C}=h_{C}\left(C_{B}\right)=\eta\left[C_{B}-C_{N E}\right]_{+},
$$

where the no-effect concentration, $C_{N E}$, is the threshold concentration below which the toxicant has no effect, and the toxicity slope $\eta$ characterizes the increase of the hazard for a unit increase of toxicant. Smaller $C_{N E}$ and higher $\eta$ imply greater toxic 
effects.

I included only effects from the toxciants in the blood, but toxicants associated with structural or energy storage lipids could have impact on the individual as well. One could account for such effects by replacing $C_{B}$ with $C_{L}$ or $C_{S}$, or adding terms with $C_{L}$ and $C_{S}$ to $h_{C}$.

\subsubsection{Characterizing bioaccumulation}

To investigate patterns of bioaccumulation, first I have to characterize it, both on individual and population level. In this model, $C_{B}$ is a natural choice for individuallevel measure of bioaccumulation because $C_{B}$ determines effects of exposure. It is not, however, a good population-level measure because of differences between individual patterns of bioaccumulation arising from differences in age, reproductive history, and the initial maternal transfer between individuals.

I use $\overline{C_{B}}$, the average $C_{B}$ among all individuals, and $\operatorname{Var}\left(C_{B}\right)$, the variance of individual $C_{B}$ among all individuals, as population-level indices of bioaccumulation. These indices, calculated for any given time, convey information on overall effects of exposure at that time: $\overline{C_{B}}$ drives the overall effects of exposure, while $\operatorname{Var}\left(C_{B}\right)$ describes the variability of these effects. The asymptotic values and trends of $\overline{C_{B}}$ and $\operatorname{Var}\left(C_{B}\right)$ describe patterns of bioaccumulation for a given exposure scenario.

When a new toxicant is introduced into the environment, or an established one is regulated, the indices of bioaccumulation change. If a toxicant is introduced in a pristine environment, the environmental toxicant concentration $C_{I}$ increases and $\overline{C_{B}}$ must increase because of bioaccumulation. If a toxicant is in the environment long enough, $\overline{C_{B}}$ approaches the asymptotic value because accumulation of toxicants through assimilation equilibrates with their export through mortality and inefficiencies of reproduction. If such a toxicant is regulated, $C_{I}$ decreases, assimilation decreases while export (at least initially) stays the same, and $\overline{C_{B}}$ must decrease as well. Eventually, $\overline{C_{B}}$ approaches an asymptotic value again. 
To determine the rates of change of $\overline{C_{B}}$ in response to changes in $C_{I}$, I approximate the inherently complex system of all individuals and their interactions which determine the dynamics of $\overline{C_{B}}$ with a linear system. Then $\overline{C_{B}}$ approaches the asymptotic value exponentially with the intrinsic rate of change $\Gamma$ :

$$
\frac{d \overline{C_{B}}}{d t}=\overline{C_{B 2}}-\Gamma \overline{C_{B}}
$$

where $\Gamma$ is the intrinsic rate of change of $\overline{C_{B}}$, and $\overline{C_{B 2}}$ is the new asymptotic value of $\overline{C_{B}}$. The general solution $\overline{C_{B}}(t)$ of (3.6) at time $t$ after the change in $C_{I}$ is:

$$
\overline{C_{B}}(t)=\overline{C_{B 2}}-\left(\overline{C_{B 2}}-\overline{C_{B}}\right) \exp (-\Gamma t)
$$

where $\overline{C_{B}}$ is the asymptotic $\overline{C_{B}}$ before the change in $C_{I}$.

In principle, $\Gamma$ could depend on whether $\overline{C_{B}}$ is increasing or decreasing, and even on the asymptotic values of $\overline{C_{B}}$. To account for the possibility that $\Gamma$ depends on whether $\overline{C_{B}}$ is increasing or decreasing, I differentiate between assimilation and depuration rates. The assimilation rate of a toxicant into a population, $\Gamma_{I}$, is the rate of increase of $\overline{C_{B}}$ upon an instantaneous increase in $C_{I}$. The depuration rate of a toxicant from a population, $\Gamma_{D}$, is the rate of decrease of an established toxicant from the population following a sudden and complete disappearance of the toxicant from the environment. The assimilation and depuration rates $\left(\Gamma_{I}\right.$ and $\left.\Gamma_{D}\right)$ describe the transients of $\overline{C_{B}}$ : the greater the rates, the faster $\overline{C_{B}}$ approaches its new asymptotic value.

\subsubsection{The individual-based model}

The simulated population is made of individuals who independently react to the environment. For every individual, I separately solve the individual model presented in Sections 3.2.1 and 3.2.2. The only interaction between individuals is the one between the mother and her calf during pregnancy and nursing. To save memory, the program does not keep track of dead individuals. 
Every individual receives a unique ID at conception to help track maternity, and becomes female with a probability of $50 \%$. I record all the state variables for every individual, and a number of population-level data: simulation time, population size, age distribution, average $C_{B}$, the variance of $C_{B}$, and the total burden in the population.

When a female conceives for the first time, I record the her time of conception and her ID. Whenever a female that has not reproduced dies, I record her time of death, time of birth and her ID. These data greatly simplify calculations of all statistics of interest during post-processing.

\section{Numerical methods}

Projecting an individual forward in time requires solving the equations in Table 3.2. These equations can be written in a vector form; if individuals were completely independent, the same equations could project the population vector forward in time.

However, in mammal populations calves depend on mothers for energy transfer. I needed to modify the vector equations to accommodate the dependence. I used the array indexing feature in Mathworks Matlab to achieve this (see Appendix B).

Mortality and reproduction are discontinuous processes determined by switches (e.g. reproduction starts only when $E_{L}>E_{R}$ ) and using Matlab's routines is impractical. Therefore, I wrote a Runge-Kutta solver (myRunge.m) that evaluated the function myWEB.m to determine the rate of change of state variables, and then called the function handleY.m at the end of each time-step to evaluate the switches and handle the mortality and reproduction. I used a fourth order Runge-Kutta method with a time step of $0.002 \mathrm{y}$. The chosen time step was short enough to accommodate the largest rate in the model $\left(\beta_{L}^{-1}=0.0027 \mathrm{y}\right)$.

Most figures in Chapter 2 and 3 require multiple simulations. For example, Figure 3-8 in section 3.3.2 required nine simulations; Figure 3-2 outlines the necessary steps.

There are three main programs for each set of simulations: the control program that changes the parameters (run_CIscenarios.m), the program that executes a simu- 
lation for one set of parameters (run_WEB_once.m), and the program that draws the figure

(draw_CIscenarios.m). The code for the example in Figure 3-2 is in the Appendix B. The code contains a short description of every procedure, and limited comments at crucial points.

\section{Initial population}

To minimize transients, I used an initial age distribution as close to the stable one as possible by using the survival-to-age distribution (3.2) to create my initial population. For moderate and high food availability $(f=0.9,0.99)$, the population consisted of 300 individuals. For low food availability $(f=0.81)$, the initial population consisted of 15,000 individuals to allow for convergence before extinction.

Due to problems of coupling a fetus or a nursling with the right mother in a randomly generated initial population, I generated the initial population using only weaned individuals. To determine the state variables assigned to each individual, I used individual data for female and male right whales from Chapter 2 as reference individuals: the state variables of each initialized individual are set equal to the state variables of the reference individual at the same age.

This method is of initialization does not give a stable age distribution. It ignores reproduction, and biases the initial distribution towards older individuals because there are no calves, and results in an initial surge of reproduction because some mothers that would otherwise be preoccupied with reproduction can accumulate energy and reproduce sooner.

Therefore, transients are inevitable. To minimize their impact on the calculation of indices of bioaccumulation, population growth rate and reproductive probability, I use data generated after the transients subsided. Visual inspection suggested that, when $C_{I}$ is constant, indices of bioaccumulation converge faster than the age distribution. Hence, I used the convergence of the age distribution as a signal that transients 

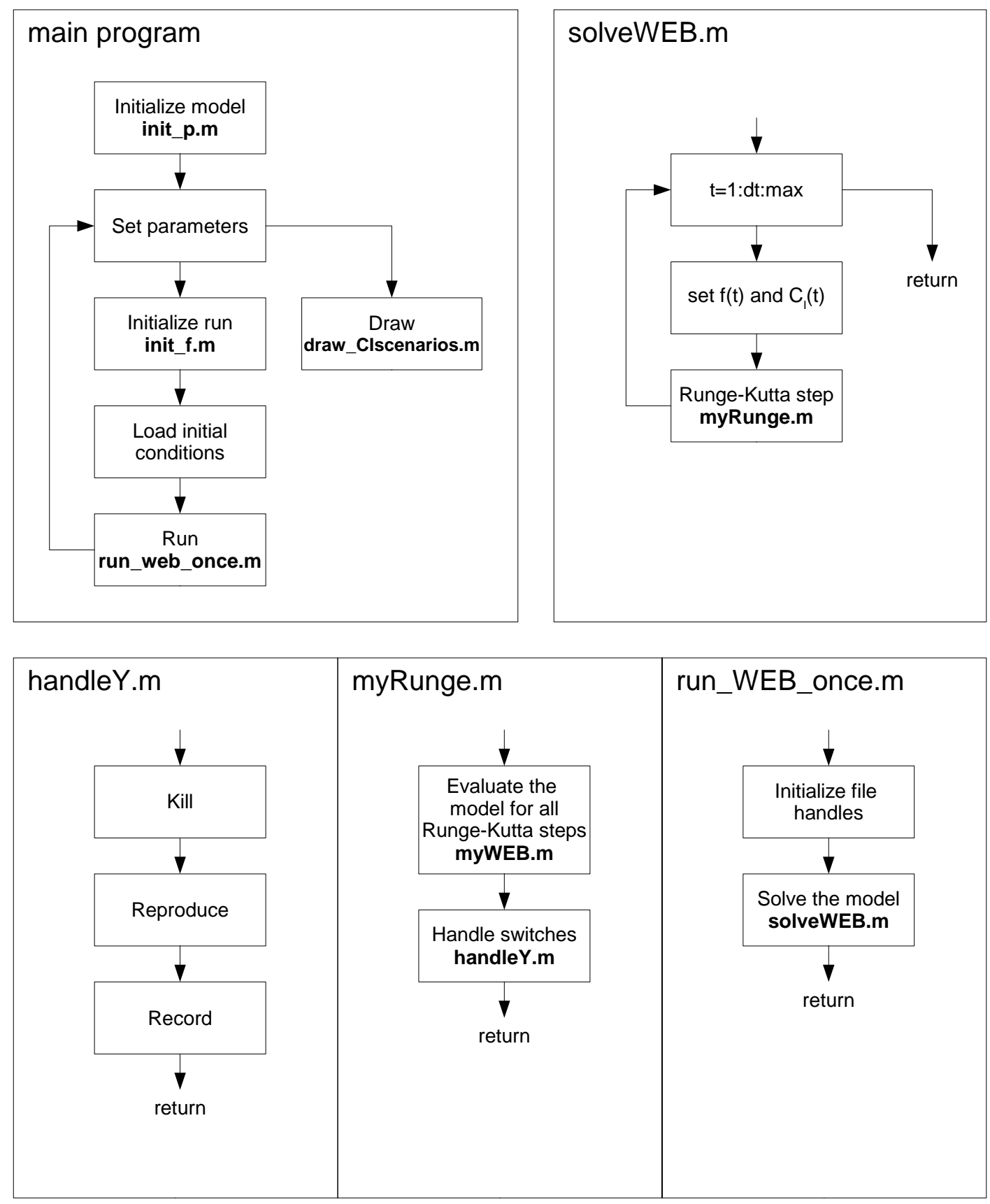

Figure 3-2: Flowchart of the main steps in the simulations. 

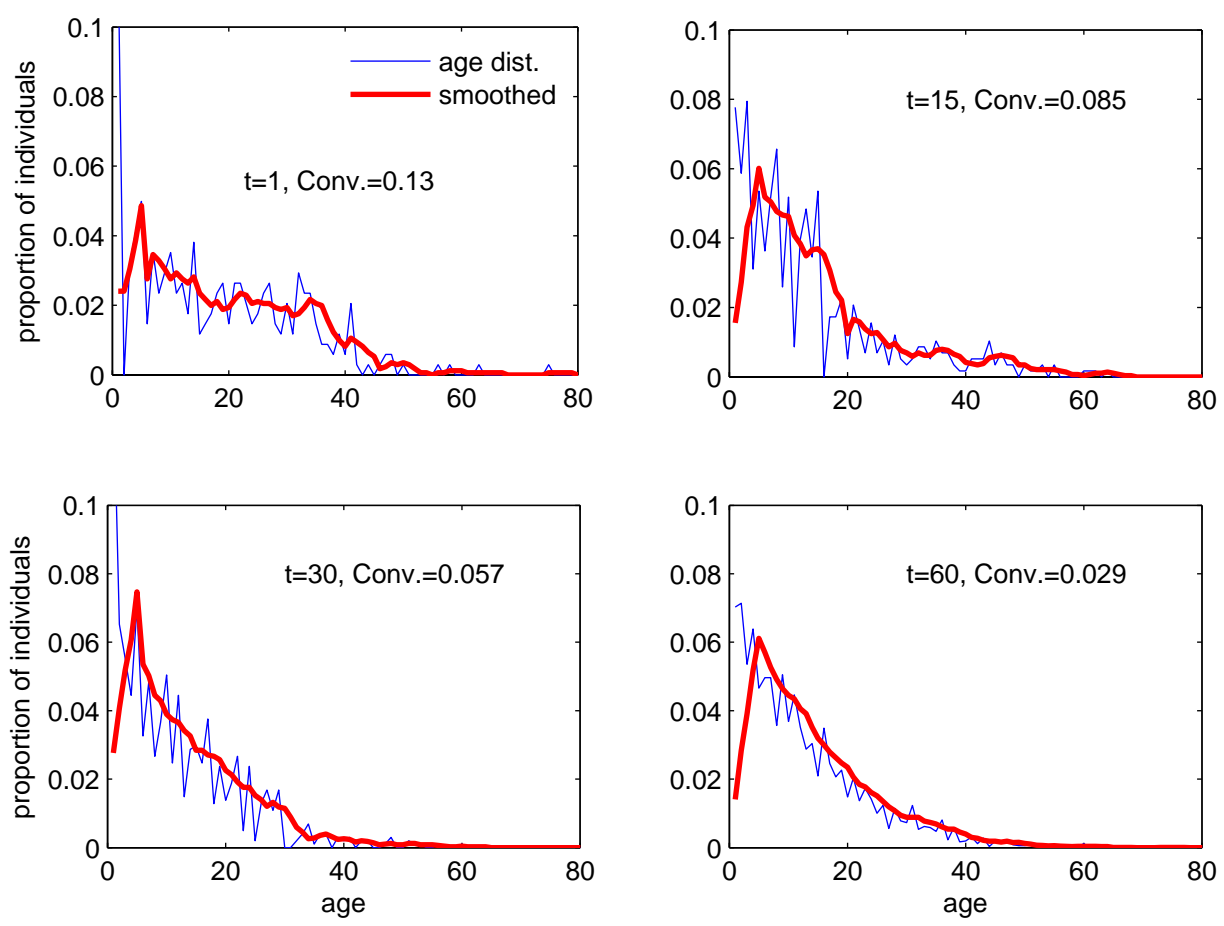

Figure 3-3: Measuring the convergence of the age distribution. The age distribution, filtered age distribution and convergence score (Conv.) shown at $t=1, t=15, t=30$ and $t=60$ for $f=0.9$ and $\eta=0$.

subsided.

\section{Calculating convergence of the age distribution}

For each year in the model run, I smoothed the age distribution by filtering it with a five-year age span (Figure 3-3). I compared the filtered age distributions between years to estimate convergence. I scored the convergence of the distribution by calculating the 1-norm the difference between the smoothed age distributions in two consecutive years. Distributions converged enough for the purposes of calculating the population growth rate when the score fell below 0.05. Complete convergence is not critical to the results because I averaged the observations from the time when the score fell below 0.05 to the end of simulation, or to the time when population size fell below 80 individuals. 


\section{Calculating the growth rate}

I use the population growth rate as an asymptotic (long-term) population-level measure of effects of toxicants. Thus I observe population size only after the distribution converges, assuming that then the population grows exponentially:

$$
N(t)=N_{0} \exp (r t),
$$

where $r$ is the population growth rate. Rearranging and taking a natural logarithm of (3.8) gives

$$
\ln \left(N(t) / N_{0}\right)=r t,
$$

which is a linear function of $t$. The left-hand side of (3.9) is one of the outputs of the model. I fit $r$ using linear regression.

\section{Calculating the probability of reproducing at least once}

I calculate the probability that a female reproduces at least once by keeping track of all newly conceived fetuses and counting how many reproduced before dying. I record a unique ID of all newly conceived female fetuses, and record when they reproduce for the first time, or when they die. The probability of reproduction is then the ratio of females that reproduced and the total number of females born at the same year.

\subsection{Results}

I use the population growth rate as an indicator of long-term consequences of exposure. I use the probability that female reproduces at least once as a measure of the effects of toxicants on a shorter, individual time scale.

In this section, I first investigate effects of exposure on bioaccumulation, population growth rate and reproductive probability. Next, I investigate effects of maternal transfer on bioaccumulation and effects of exposure. 

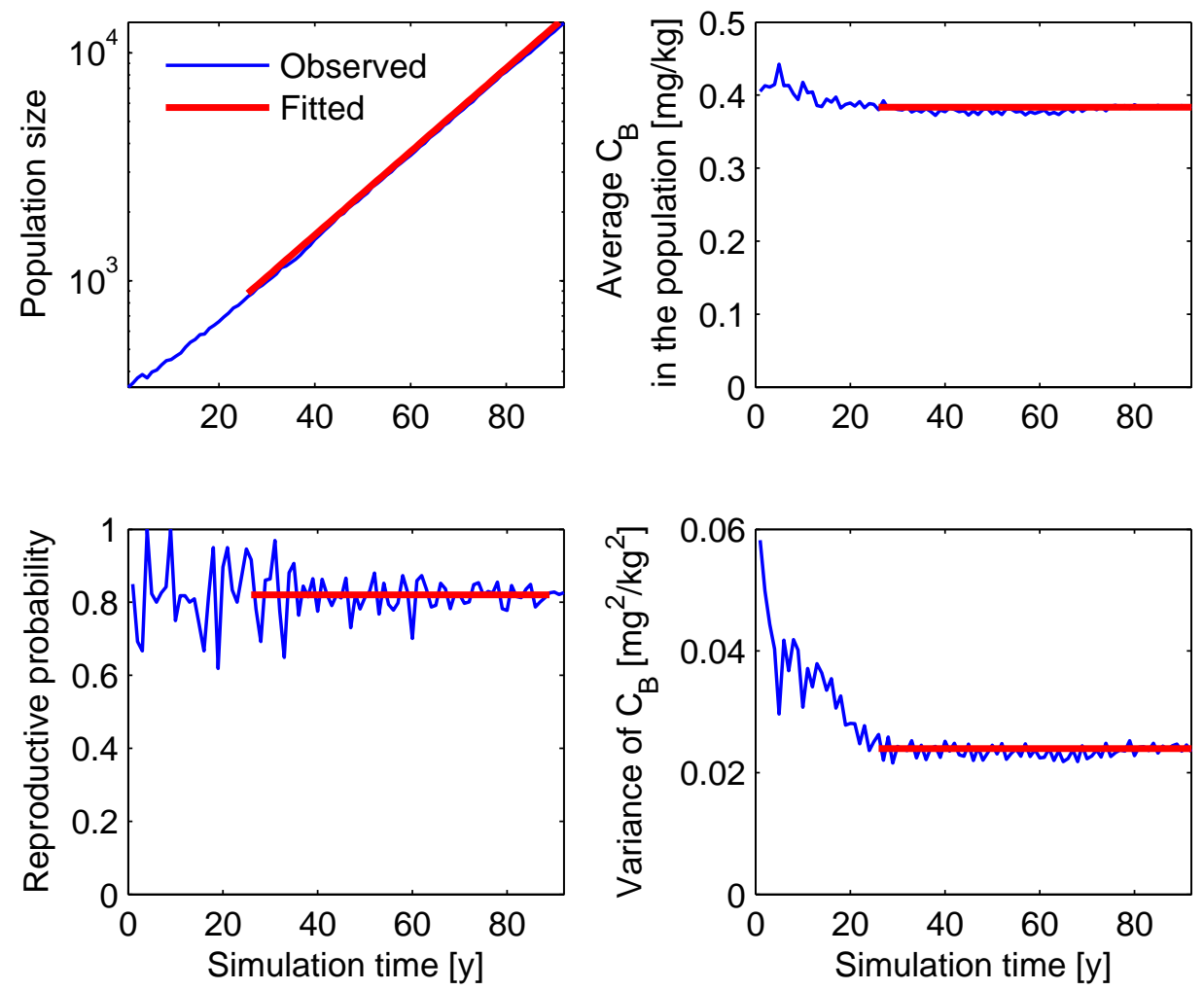

Figure 3-4: Outputs of the population model during one simulation. Left: population size (top) and reproductive probability (bottom). Right: $\overline{C_{B}}$ (top) and $\operatorname{Var}\left(\overline{C_{B}}\right)$ (bottom). Population growth rate, reproductive probability, $\overline{C_{B}}$ and $\operatorname{Var}\left(\overline{C_{B}}\right)$ have been estimated using observations after $t=28$ (red line). Simulations in this figure are for $f=0.9, \eta=0.05 \mathrm{~kg} \cdot \mathrm{mg}^{-1} \mathrm{y}^{-1}$, and $C_{N E}=0.5 \mathrm{mg} / \mathrm{kg}$. 
I simulate the IBM for three food availabilities $(f)$ and a number of toxicities of the toxicant ( $\eta$ and $C_{N E}$ ). I observe effects of these parameters on bioaccumulation, effects of exposure, population growth rate, and reproductive probability. Figure 3-4 shows a typical simulation.

\subsubsection{Effects of exposure on bioaccumulation and population growth rate}

The effect of toxicants on individuals is determined by the toxicant action model (3.5), parameterized by $\eta$ and $C_{N E}$. These parameters differ between toxicants: higher $\eta$ and lower $C_{N E}$ imply higher potency. To investigate how this difference affects populations, I varied $\eta$ and $C_{N E}$ and observed population growth rate, reproductive probability, and average $C_{B}$ and its variance.

I varied $\eta$ between no effect and a large effect, $\eta \in[0,0.05,0.1,0.5,1] \mathrm{kg} \cdot \mathrm{mg}^{-1} \mathrm{y}^{-1}$. I chose $C_{N E}$ so that it falls beneath, at, and above the expected terminal female $C_{B}$ of $0.3-0.4 \mathrm{mg} / \mathrm{kg}$ observed in Chapter $2, C_{N E} \in[0.25,0.4,0.5] \mathrm{mg} / \mathrm{kg}$.

Results from Chapter 2 suggest that food availability affects bioaccumulation of individuals. To investigate how it affects the populations, I repeated the simulations for three values of $f: f_{1}=0.81, f_{2}=0.9$ and $f_{3}=0.99$. Food availabilities $f_{2}$ and $f_{3}$ correspond to those experienced by North Atlantic and southern right whales; $f_{1}$ is $10 \%$ lower than $f_{2}$.

Figure 3-5 shows the results. As expected, the population growth rate and the probability of successful reproduction is smaller for more potent toxicants for all values of $f$ (upper and lower left plots). The effect is more pronounced for lower food availability due to higher $\overline{C_{B}}$ associated with lower $f$ (upper right plot).

When $f$ is low, it takes a longer time to mature and recover from a reproductive events. During that time, individuals have to maintain their structure and bioaccumulate toxicants as a result. Hence, longer times to maturity and longer calving intervals imply higher $\overline{C_{B}}$. The effects of exposure reduce average $C_{B}$ and its variance 

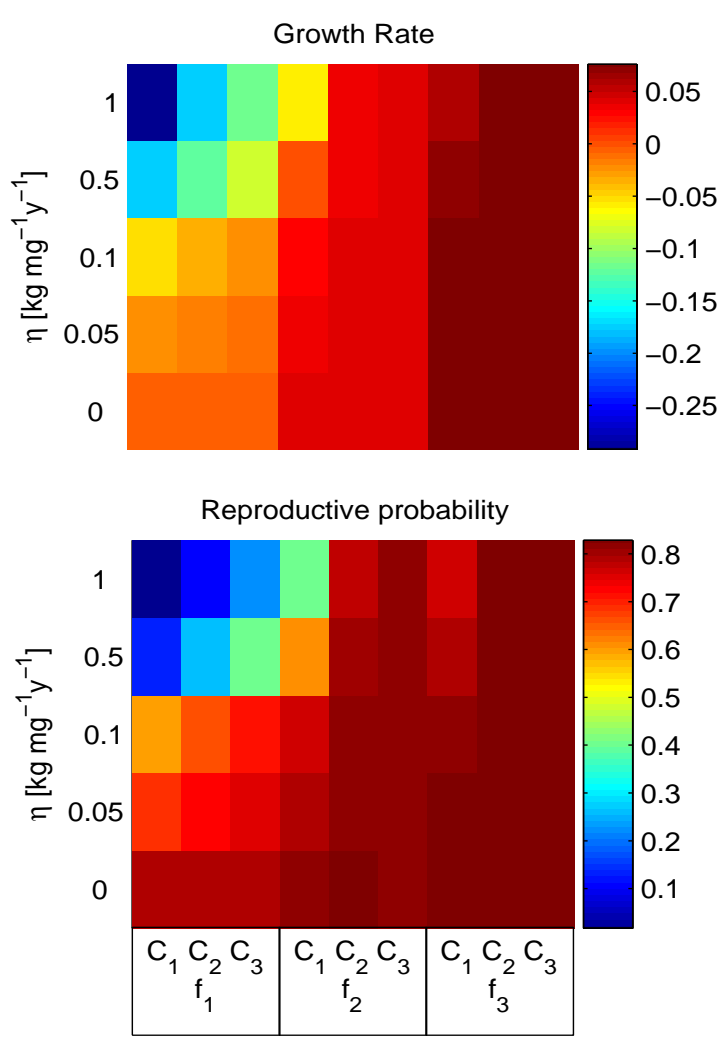

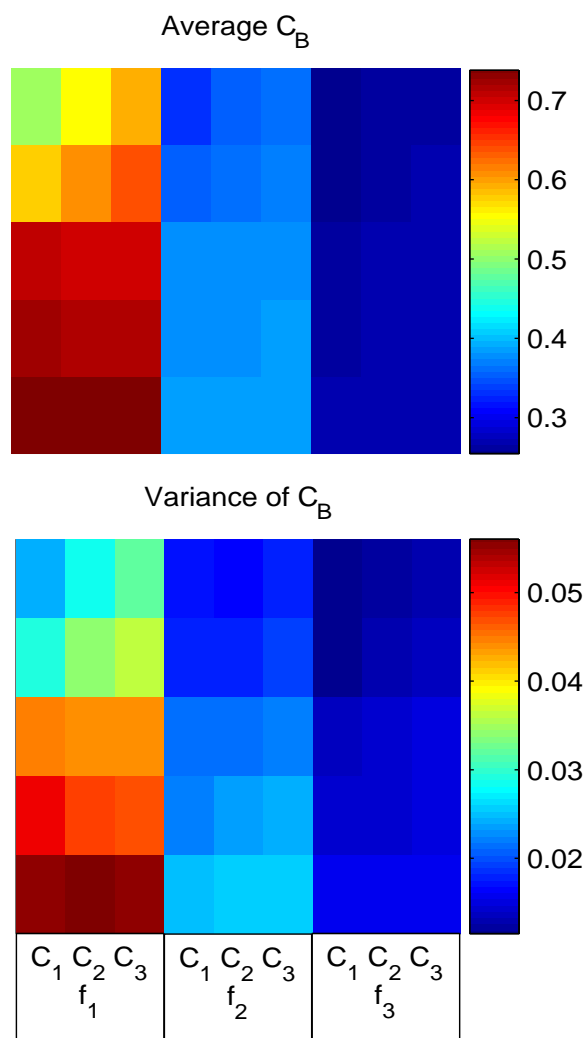

Figure 3-5: Effects of exposure on population growth rate and bioaccumulation. Left: population growth rate (top) and reproductive probability (bottom). Right: average $C_{B}$ (top) and variance of $C_{B}$ (bottom) for three food availabilities $\left(f_{1}=0.81, f_{2}=0.9\right.$ and $\left.f_{3}=0.99\right)$, three values of $C_{N E}\left(C_{1}=0.25, C_{2}=0.4\right.$ and $\left.C_{3}=0.5\right)$ and a range of $\eta$ (see text for description and parameter values). The plotted values are listed in the Appendix A. 
because individuals with higher $C_{B}$ are preferentially removed from the population.

When $\eta=0$, there are no effects of exposure for any value of $C_{N E}$, and the toxicant behaves like a passive tracer. The resulting population growth rates reflect the maximum potential growth rate for a given $f$. This implies that the North Atlantic right whale population $(f=0.9)$ has the potential to increase about $4 \%$ annually, while the southern right whale population $(f=0.99)$ has the potential to increase about $8 \%$ annually.

\subsubsection{Effects of maternal transfer on bioaccumulation and effects of exposure}

The three basic modes of maternal transfer mentioned in section 3.2.1 are complete transfer $(\mathrm{M}-\mathrm{C}+)$, mother's loss $(\mathrm{M}-\mathrm{C} 0)$, and no-transfer $(\mathrm{M} 0 \mathrm{C} 0)$ modes. These modes by definition affect individual exposure and bioaccumulation. Transfer $(\mathrm{M}-\mathrm{C}+)$ and no-transfer $(\mathrm{M} 0 \mathrm{C} 0)$ modes are opposites, and comparing their effects can provide an insight into the effects of maternal transfer. Figure 3-6 compares the long-term effects of maternal transfer by showing differences in population growth rate, reproductive probability, and measures of bioaccumulation with $(\mathrm{M}-\mathrm{C}+)$ and without (M0C0) transfer of toxicants.

The maternal transfer increases $\overline{C_{B}}$ by about $40 \%-50 \%$ (upper right plot). When transferring toxicants, mothers reduce their $C_{B}$, but increase $C_{B}$ of calves at weaning as a result. The increase of $C_{B}$ at weaning on average trumps the reduction due to transfer. Recycling of toxicants by passing them to the next generation through maternal transfer further increases $\overline{C_{B}}$. The $30 \%$ loss of toxicants during reproduction due to discarded materials (e.g. placenta) and fecal excretion of the nursling is not sufficient to make up for the recycling.

When there is no maternal transfer, individuals start without any burden, and consequently bioaccumulate less before their first reproduction. When toxic effects are important (high $\eta$, low $C_{N E}$ ), the probability of reproducing before dying is, therefore, 

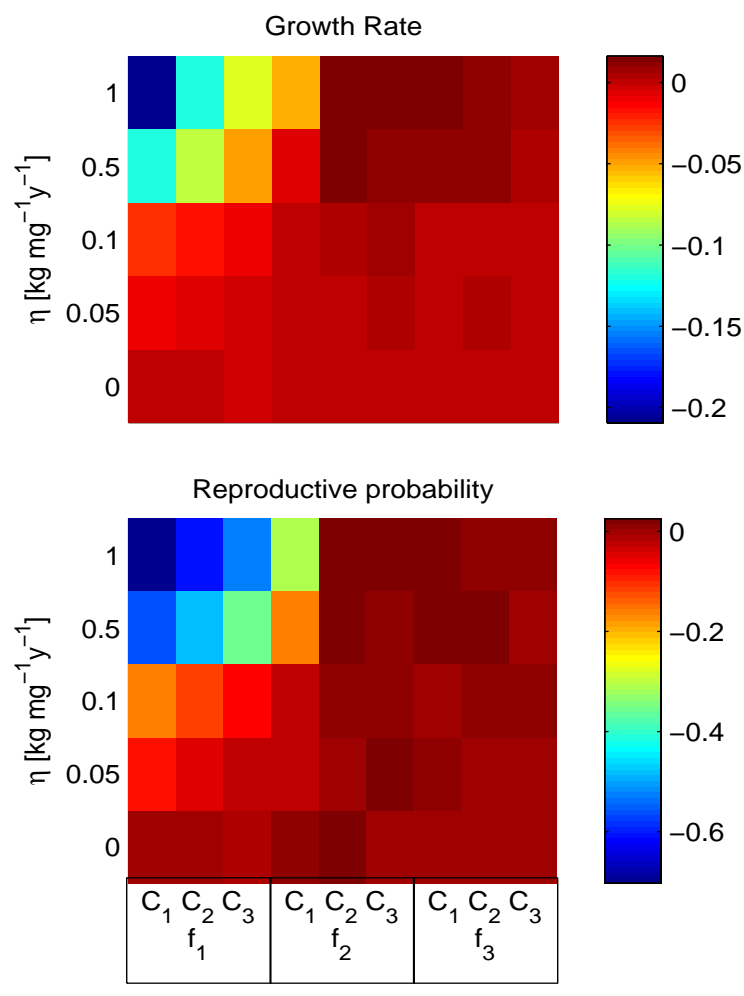

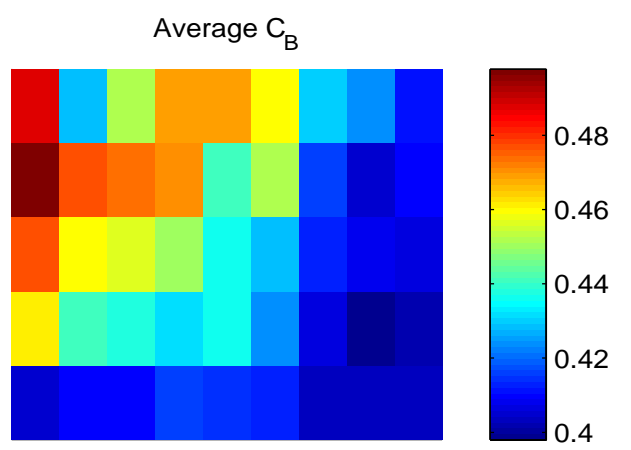

Variance of $C_{B}$

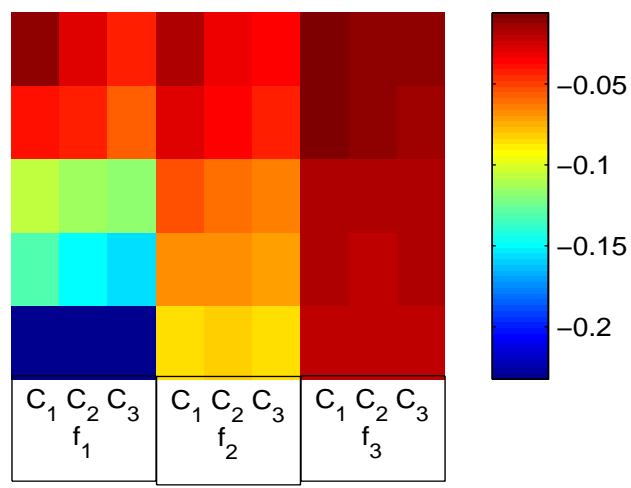

Figure 3-6: Effects of maternal transfer on population growth rate and bioaccumulation. Left: the change in population growth rate (top) and the decrease in reproductive probability (bottom) due to maternal transfer. Right: proportional increase in average $C_{B}$ (top) and decrease in variance of $C_{B}$ (bottom). All plots drawn for three food availabilities $\left(f_{1}=0.81, f_{2}=0.9\right.$ and $\left.f_{3}=0.99\right)$, three values of $C_{N E}\left(C_{1}=0.25\right.$, $C_{2}=0.4$ and $\left.C_{3}=0.5\right)$ and a range of $\eta$. The model is stochastic because mortality is stochastic, so small variations between simulations are to be expected. The plotted values are listed in the Appendix A. 


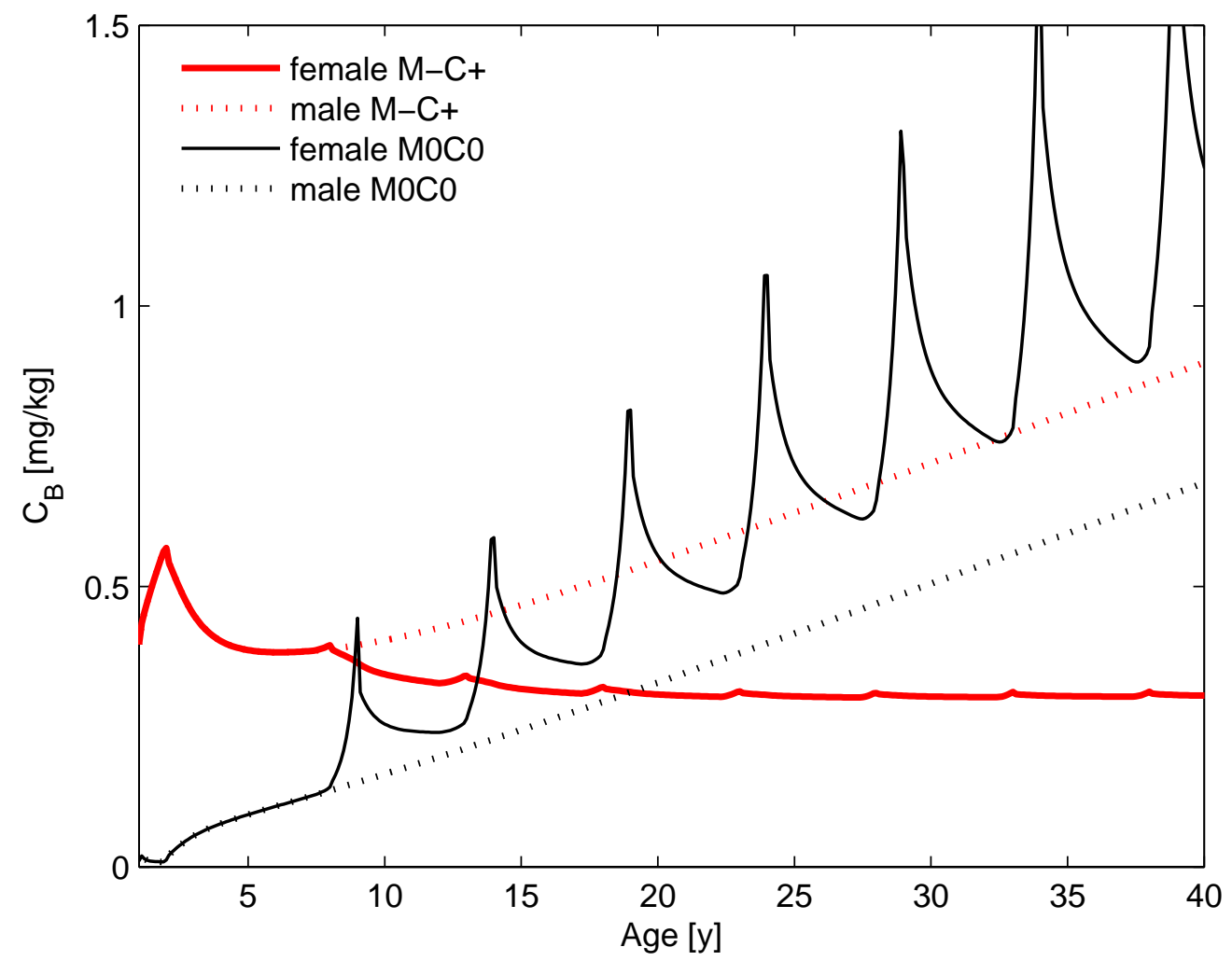

Figure 3-7: Bioaccumulation with and without maternal transfer. Mothers that do not transfer toxicants have much higher $C_{B}$ then either males or females that transfer toxicants. Energy demands of reproduction further increase $C_{B}$ of mothers that do not transfer toxicants.

higher with no maternal transfer (Figure 3-6, lower left plot). As $f$ decreases and the toxic effects increase (higher $\eta$, lower $C_{N E}$ ), effects of exposure become important, and the maternal transfer reduces the probability of reproducing by up to $50 \%$.

Despite decreasing probability of reproducing and increasing $\overline{C_{B}}$ for all toxicities, toxicant transfer may increase population growth rate for some combinations of food availability and toxicity. For $f=0.99$, maternal transfer increases the population growth rate for all toxicities investigated. For $f=0.91$, maternal transfer increases the growth rate except for the highest toxicity (see table 3.10 in the Appendix A). If 
the maternal transfer increases the population growth rate, it increases it more when toxicity is higher.

While males who did not assimilate toxicants during nursing always have lower $C_{B}$ than males who did, the reverse is true for females after a few reproductive events (Figure 3-7): even though they start from $C_{B}$ of almost zero and suffer smaller initial mortality, mothers who do not transfer toxicants to their calves reach the $C_{B}$ of mothers that transfer their burden by the time they have a second calf. During reproduction, mothers that do not transfer toxicants drastically reduce their energy storage, but not their toxicant burden. If they reproduce more than twice, this dramatically increases their $C_{B}$ (Figure 3-7) until they restore energy reserves.

The resulting increases in $C_{B}$ of mothers that do not transfer toxicants increase $h_{C}$ and shorten their reproductive lifetime, which in turn reduces the population growth rate. The reduction of the population growth rate is more significant as toxic effects increase.

On the other hand, if the population growth rate is small, maternal transfer further decreases it. Then, females reproduce only a small number of times in either maternal transfer mode, and the adverse effects of no maternal transfer cannot manifest.

Hence, if the population experiences food abundance, toxicants that are maternally transferred can have positive impact on the population; if the population is already food-stressed, maternally transferred toxicants put them in further jeopardy. I expect that the increase in the population growth rate due to maternal transfer is more significant in species with longer reproductive span and more reproductive events because in such species females lose more reproductive events if they die early.

The increases of the variance of $C_{B}$ (Figure 3-6, lower right plot) is indicative of the sharp increase in $C_{B}$ of females that do not transfer toxicants. This is more pronounced for lower values of $f$. When there is no toxic effect $(\eta=0)$, the variance without maternal transfer is greater by about 5 times for $f=0.81,4.3$ times for $f=0.9$, and 2.5 times for $f=0.99$ than the variance with the maternal transfer. 
Increasing the potency of the toxicant decreases the variance (as well as the difference of the variance) because it preferentially removes individuals with high burdens from the population.

\section{Transients: assimilation $\left(\Gamma_{I}\right)$ and depuration $\left(\Gamma_{D}\right)$ rates}

To further investigate effects of maternal transfer on bioaccumulation, I compared transients of average $C_{B}$ and its variance in all three food availabilities for three modes of maternal transfer $(\mathrm{M}-\mathrm{C}+, \mathrm{M}-\mathrm{C} 0$ and $\mathrm{M} 0 \mathrm{C} 0)$. I modeled the two scenarios that define $\Gamma_{I}$ and $\Gamma_{D}$ corresponding to introduction and regulation of a toxicant.

The first, "step up" scenario models a sudden introduction of the toxicant into the environment: $C_{I}$ changes from zero to $0.035 \mathrm{mg} / \mathrm{kg} 40$ y into the simulation. In the second, "step down" scenario, I model a removal of a toxic substance from the environment: $C_{I}$ drops from $0.035 \mathrm{mg} / \mathrm{kg}$ to zero at $40 \mathrm{y}$ into the simulation. I did not incorporate effects of exposure $(\eta=0)$.

Figure 3-8 shows the average $C_{B}$ and its variance for $f=0.9$. In both scenarios, the average toxicant concentrations are highest when mothers transfer toxicants $(\mathrm{M}-\mathrm{C}+)$ because of the recycling of toxicants due to maternal transfer. When mothers lose but calves do not assimilate toxicants $(\mathrm{M}-\mathrm{C} 0)$, the bioaccumulation is least prominent because the toxicant is exported from the population at a high rate. The no-transfer mode $(\mathrm{M} 0 \mathrm{C} 0)$ falls between the other two modes. I observed the same patterns for the other two values of $f$ (not shown).

I measured $\Gamma_{I}$ and $\Gamma_{D}$ for all food availabilities and modes of transfer (Figure 3-9). To calculate $\Gamma_{I}$, I fitted the $\overline{C_{B}}(t)$ observed in the step up scenario at time $t$ after the introduction of toxicants to (3.7) for $\overline{C_{B 1}}=0$, and $\overline{C_{B 2}}=a$ :

$$
\overline{C_{B}}(t)=a\left(1-\exp \left(-\Gamma_{I} t\right)\right)
$$

To calculate $\Gamma_{D}$, I fitted the $\overline{C_{B}}(t)$ observed in the step down scenario after the 

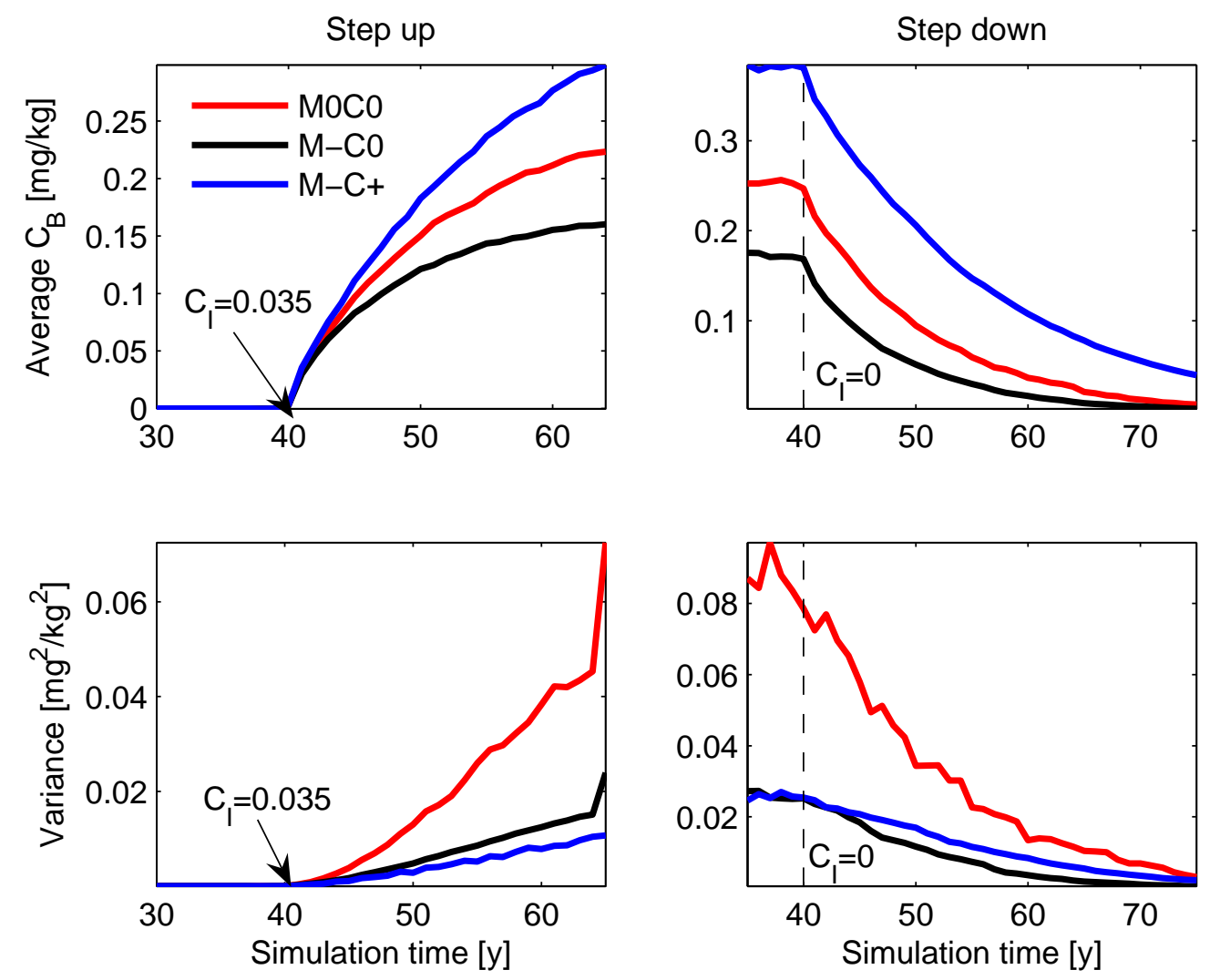

Figure 3-8: Transient response of bioaccumulation to changes in $C_{I} . \overline{C_{B}}$ (upper left plot) and $\operatorname{Var}\left(C_{B}\right)$ (lower left plot) for step up, and $\overline{C_{B}}$ (upper right plot) and $\operatorname{Var}\left(C_{B}\right)$ (lower right plot) for step down scenarios of changes in $C_{I}$ for three maternal toxicant transfer modes (M0C0, M-C0, M-C+), see text for details. 

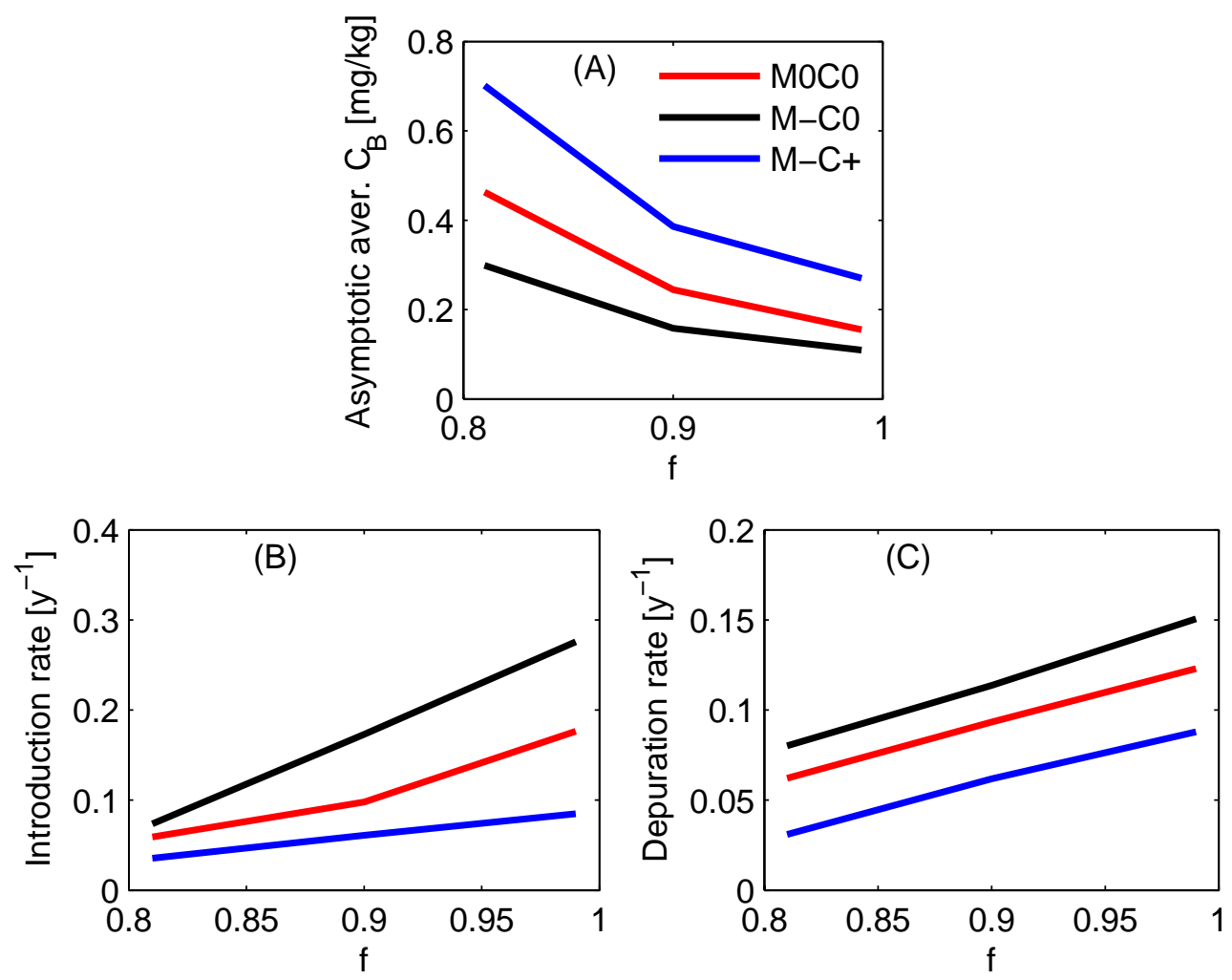

Figure 3-9: Dependance of transients on food availability. Asymptotic average $C_{B}$ (A), Introduction rate (B), and depuration rate (C) for three maternal toxicant transfer modes (see text for details). 
regulation of a toxicant, to $(3.7)$ for $\overline{C_{B 1}}=a$, and $\overline{C_{B 2}}=0$ :

$$
\overline{C_{B}}(t)=a \exp \left(-\Gamma_{D} t\right)
$$

I estimated $a$ from the asymptotic $\overline{C_{B}}$ when $C_{I}$ was greater than zero, and fitted both rates using linear regression.

The assimilation and depuration rates increase with food level. For the North Atlantic right whale, the depuration rate is $0.06 \mathrm{y}^{-1}$ assuming maternal transfer $(\mathrm{M}-\mathrm{C}+)$, and $30 \%$ higher if the mother retains the toxicant (M0C0). As observed in Section 3.3.1, the asymptotic $\overline{C_{B}}$ decreases as $f$ increases. The asymptotic $\overline{C_{B}}$ is about 2.3 times larger for $\mathrm{M}-\mathrm{C}+$ maternal transfer mode than for $\mathrm{M}-\mathrm{C} 0$ mode, and about 1.5 times larger than for the M0C0 mode. The difference between $\mathrm{M}-\mathrm{C} 0$ and M0C0 modes is larger than the previously observed $40 \%$ increase (Figure 3-6). This is probably because I did not include effects of exposure in the simulations which

tend to decrease $\overline{C_{B}}$ by preferentially removing individuals with high $C_{B}$ from the population.

The assimilation rates are surprisingly similar to depuration rates for every food level and maternal transfer mode. Both types of rates increase with food level and depend on the mode of maternal transfer. All individuals in the $\mathrm{M}-\mathrm{C} 0$ and $\mathrm{M} 0 \mathrm{C} 0$ mode undergo the same bioaccumulation scenario, which leads to faster transient responses of $\overline{C_{B}}$ to changes in $C_{I}$ compared to the responses $\mathrm{M}-\mathrm{C}+$ mode, in which recycling slows the transients.

\subsection{Discussion}

Understanding how maternal transfer of toxicants affects bioaccumulation and how the resulting exposure affects population growth rate is a necessary step toward better management and regulation of toxicants. To investigate these linkages, one has to be able to observe population growth rate and bioaccumulation as a function of mater- 
nal transfer, energy availability, environmental toxicant concentration and effects of exposure. To this end, I developed a population model based on the individual model presented in Chapter 2. My most important results were:

1. Higher toxic effect (higher $\eta$, lower $C_{N E}$ ) decreases the average concentration and variance of toxicants in the blood due to preferential removal of individuals with high concentrations of toxicants.

2. Maternal transfer decreases probability of reproducing at least once.

3. Maternal transfer increases population growth rate of right whales; the increase is greater for higher toxic effect.

4. Transient responses to changes in environmental toxicant concentrations can be described by assimilation and depuration rates.

5. Assimilation rate and depuration rate of a toxicant for a population in a specific environment are equal for practical purposes. Recycling of toxicants through maternal transfer reduces the rates.

6. Energy availability allows for North Atlantic right whale population growth of $4 \%$, and southern right whale population growth of about $8 \%$ annually.

7. Depuration rate of lipophilic toxicants from a right whale population (neglecting biotransformation) is $0.06-0.09 \mathrm{y}^{-1}$.

Even though I used a model adapted to the right whale, the approach - and most of the results - are applicable to other marine mammals. For example, results 1 and 2 are a consequence of effects of exposure, and not specific to right whales. If a high concentration of toxicants in the blood $\left(C_{B}\right)$ reduces survival probability, individuals with higher $C_{B}$ are more likely to die and thereby reduce the average toxicant concentration. Similarly, individuals that have not received any burden 
from their mothers have smaller $C_{B}$, are more likely to live, and are consequently more likely to reach a reproductive age.

The same mechanism may be responsible for the somewhat surprising result 3 : mothers that do not transfer the toxicants to their young will live shorter and reproduce less. The effect on the population growth rate is greater when females reproduce often, and reproduce many times. This implies that species with long reproductive life span and frequent reproductive events are better off transferring toxicants to their young, while the opposite is true for those with a short reproductive life span.

I investigated transients for three modes of maternal toxicant transfer. The transient response rates exhibit same pattern of dependence on food availability when effects of toxicants are ignored. The patterns may, however, depend on the effects of exposure. I plan to investigate this in the future.

The results have implication for regulation of toxic substances. First, the fairly slow rates of assimilation $\left(\Gamma_{I}\right)$ imply that short-term monitoring programs may not be sufficient to estimate long-term bioaccumulation patterns of a toxicant. Second, because rates are so slow, toxicant may not have reached its asymptotic value when $C_{I}$, the toxicant concentration in the environment decreases. In that case, $\overline{C_{B}}$ may still increase if the asymptotic value of $\overline{C_{B}}$ for the new $C_{I}$ is higher than $\overline{C_{B}}$ at the time of regulation. Third, the increase in $\overline{C_{B}}$ due to maternal transfer may have significant consequences for highly toxic substances, and should be taken into account when regulating persistent toxicants that can be transferred during reproduction. For example, $\overline{C_{B}}$ increases by $40-50 \%$ in right whales due to maternal transfer; this increase exposure may be fatal. Single-generation studies, which are used almost exclusively in legislature (U.S. EPA 1996), do not take this increase into the account and may, therefore, overestimate 'safe' environmental toxicant concentrations $\left(C_{I}\right)$. Because of very slow depuration rate of maternally transferred persistent toxicants from populations, even a quick regulation of the toxicant may not help alleviate adverse effects once they are noticeable. Therefore, it is important to account for the 
possible increase in average $\overline{C_{B}}$ when relying on single-generational studies.

Variance of $C_{B}$ is important as well: even if the $\overline{C_{B}}$ is small, large variability suggests that $C_{B}$ of many individuals may be large. In some cases, this may not be acceptable.

Our estimate for southern right whale population growth potential of $8 \%$ annually (result 6) is only slightly larger than their measured growth rate of $7 \%$ annually (Best et al. 2001). Such a small difference suggests that the southern right whales do not experience many sources of mortality in addition to their natural mortality. The estimate is, however, based on the right whale life expectancy of 25 years; southern right whales may have a longer life expectancy. To correctly asses the potential population growth rate, I would need to adapt the model to the southern right whales.

The estimated potential for North Atlantic right whale population growth rate of $4 \%$ is in stark contrast with the observed, slightly negative one (Fujiwara and Caswell 2001). The difference in potential population growth rates between North Atlantic and southern right whales suggests that food availability plays a significant role in North Atlantic right whale population growth. The unrealized fairly large potential North Atlantic right whale population growth rate of $4 \%$, on the other hand, suggests that stressors other than the lack of food, such as effects of toxicants and ship strikes, prevent North Atlantic right whale population recovery.

Authors have suggested that sublethal exposure to toxicants may be a contributing reason for the North Atlantic right whale decline (Knowlton et al. 1994, Angell 2005). The depuration rate of persistent toxicants from the right whale population (result 7) is very slow. This implies that any regulation of possibly harmful substances can help the right whales only in the long run. Therefore, controlling pollution - while beneficial in the long run - is not likely to mitigate effects of persistent toxicants on North Atlantic right whales in the short run.

Results in this chapter rely on the assumed toxicant action model; results may be different for other toxicant action models. For example, toxicants can also affect 
reproduction (e.g. Addison 1989, Reijnders 1986). Both direct and indirect effects of toxicants on reproduction can readily be incorporated in the model. Direct effects include an increase in energy required for reproduction $\left(E_{R}\right)$ or decrease in reproductive efficiency $\left(k_{R}\right)$. Increasing maintenance costs $(m)$ or reducing foraging ability $\left(I_{\max }\right)$ could have indirect effects on reproduction because it reduces energy available for reproduction and increases time needed to recuperate from a reproductive event. All of these effects may increase bioaccumulation and induce further negative effects.

I assume that individuals are equally susceptible to toxicants in all life stages, but susceptibility to toxicants can be life-stage dependent. Many toxicants influence development (e.g. Reijnders and Brasseur 1992, Guo et al. 2004) and pre-pubescent individuals more susceptible to toxicants than already developed individuals. In that case, maternal transfer could have a much greater negative impact on population growth rate than currently predicted by my model. Likewise, older individuals may be more susceptible. In that case, mothers that retain the toxicants may succumb to toxicants even sooner than in the current model. This would also reduce the population growth rate. Using the model presented in this chapter, one can change the effects of exposure to investigate how food availability and life-stage dependent susceptibility to toxicants interact to affect population growth rate.

\subsection{References}

Addison, R. F. 1989. Organochlorines and marine mammal reproduction. Canadian Journal of Fisheries and Aquatic Sciences, 46, 360-368.

Aguilar, A., and A. Borrell. 1991. Heterogeneous distribution of organochlorine contaminants in the blubber of baleen whales: Implications for sampling procedures. Marine environmental research 31:275-286.

Aguilar, A., and A. Borrell. 1994. Reproductive transfer and variation of body load of organochlorine pollutants with age in fin whales (Balaenoptera physalus). 
Archives of Environmental Contamination and Toxicology 27:546-554.

Angell, C.M. 2005. Body Fat Condition of Right Whales, Eubalaena glacialis and Eubalaena

australis. PhD. Thesis, Boston University, Boston, MA.

Best, P.B., A. Brandao, and D.S. Butterworth. 2001. Demographic. parameters of southern right whales off South Africa. Journal of Cetacean Research and Management Special Issue 2:161-169

Boon, J., I. Oostingh, J. van der Meer, and T. Hillebrand. 1994. A Model fo the bioaccumulation of chlorobiphenyl congeners in marine mammals. European Journal of Pharmacology: Environmental Toxicology and Pharmacology 270:237-251.

Borga, K., A. Fisk, P. Hoekstra, and D. Muir. 2004. Biological and chemical factors of importance in the bioaccumulation and trophic transfer of persistent organochlorine contaminants in Arctic marine food webs. Environmental Toxicology and Chemistry 23:2367-2385.

Cox, D.R. and Oakes, D. 1984. Analysis of survival data. Chapman and Hall.

de Swart, R. L., P. S. Ross, L. J. Vedder, H. H. Timmerman, S. Heisterkamp, H. Van Loveren, J. G. Vos, P. J. H. Reijnders, and A. D. M. E. Osterhaus. 1994. Impairment of immune function in harbour seals (Phoca vitulina) feeding on fish from polluted waters. Ambio 23:155-159.

Emond, C., J. Michalek, L. Birnbaum, and M. DeVito. 2005. Comparison of the use of a physiologically based pharmacokinetic model and a classical pharmacokinetic model for dioxin exposure assessments. Environmental Health Perspectives $113: 1666-1668$.

Fujiwara, M., and H. Caswell. 2001. Demography of the endangered North Atlantic right whale. Nature 414:537-541.

Guo, Y., G. Lambert, C. C. Hsu, and M. M. Hsu. 2004. Yucheng: health effects of prenatal exposure to polychlorinated biphenyls and dibenzofurans. International Archives of Occupational and Environmental Health 77:153-158. 
Hickie, B. E., M. C. S. Kingsley, P. V. Hodson, D. C. G. Muir, P. Beland, and D. Mackay. 2000. A modellingbased perspective on the past, present, and future polychlorinated biphenyl contamination of the St. Lawrence beluga whale (Delphinapterus leucas) population. Canadian Journal of Fisheries and Aquatic Sciences $57: 101-112$.

Hickie, B. E., D. Mackay, and J. De Koning. 1999. Lifetime pharmacokinetic model for hydrophobic contaminants in marine mammals. Environmental Toxicology and Chemistry 18:2622-2633.

Knowlton, A.R., S.D. Kraus and R.D. Kenney 1994. Reproduction in North Atlantic right whales (Eubalaena-glacialis). Canadian Journal of Zoology 72:12971305.

Kooijman, S., and R. van Haren. 1990. Animal energy budgets affest the kinetics of xenobiotics. Chemosphere 21:681-693.

Kooijman, S. A. L. M. 2000. Dynamic energy and mass budgets in biological systems, 2nd edition. Cambridge University press, Cambridge, UK. ISBN 0521 786088.

Lai, T. J., Y. L. Guo, N. W. Guo, and C. C. Hsu. 2001. Effect of prenatal exposure to polychlorinated biphenyls on cognitive development in children: a longitudinal study in Taiwan. The British Journal of Psychiatry 178:s49 - s52.

Lee, J. S., S. Tanabe, H. Umino, R. Tatsukawa, and D. C. Loughlin, T. R. Calkins. 1996. Persistent organochlorines in steller sea lion (Eumetopias jubatus) from the bulk of Alaska and the Bering Sea, 1976-1981. Marine Pollution Bulletin 32:535-544.

Muller, E. B., and R. M. Nisbet, 1997. Modeling the effect of toxicants on the parameters of dynamic energy budget models. in ASTM Special Technical Publication. ASTM, Conshohocken, PA, (USA).

Reijnders, P. J. H. 1986. Reproductive failure in common seals feeding on fish from polluted coastal waters. Nature 324:456 - 457 .

Reijnders, P. J. H. and Brasseur, S. M. J. M. 1992. Xenobiotic induced hormonal 
and associated developmental disorders in marine organisms and related effects in humans, an overview. In T. Colborn, \& C. Clement (Eds.) Advances in modern environmental toxicology 21:131-146.

Restum, J., S. Bursian, J. Giesy, J. Render, W. Helferich, E. Shipp, D. Verbrugge, and R. Aulerich. 1998. Multigenerational study of the effects of consumption of PCBcontaminated carp from Saginaw Bay, Lake Huron, on mink. 1. Effects on mink reproduction, kit growth and survival, and selected biological parameters. Journal of Toxicology and Environmental Health A 54:343-375.

Ross, P. S., R. L. De Swart, R. F. Addison, H. Van Loveren, J. Vos, and A. Osterhaus. 1996. Contaminant-induced immunotoxicity in harbour seals: Wildlife at risk? Toxicology 112:157-169.

Schwacke, L. H., E. O. Voit, L. J. Hansen, R. S. Wells, G. B. Mitchum, A. A. Hohn, and P. A. Fair. 2002. Probabilistic risk assessment of reproductive effects of polychlorinated biphenyls on bottlenose dolphins (Tursiops truncatus) from the Southeast United States coast. Environmental Toxicology and Chemistry 21:27522764 .

U.S. Environmental Protection Agency (1996) PCBs: Cancer Dose-Response Assessment and Application to Environmental Mixtures. National Center for Environmental Assessment, Office of Research and Development, Washington, DC, EPA/600/P-96/001F.

Wells, R., V. Tornero, A. Borrell, A. Aguilar, T. Rowles, H. Rhinehart, S. Hofmann, W. Jarman, A. Hohn, and J. Sweeney. 2005. Integrating life-history and reproductive success data to examine potential relationships with organochlorine compounds for bottlenose dolphins (Tursiops truncatus) in Sarasota Bay, Florida. Science of the Total Environment 349:106-119. 


\subsection{Appendix A - values used in figures}

\subsubsection{Figure 3-5}

Table 3.5: Figure 5: Growth rate

\begin{tabular}{|c|c|c|c|c|c|c|c|c|c|}
\hline$\eta$ & \multicolumn{9}{|c|}{ Growth rate } \\
\hline 1 & -0.29 & -0.18 & -0.12 & -0.06 & 0.03 & 0.04 & 0.06 & 0.08 & 0.08 \\
\hline 0.5 & -0.17 & -0.12 & -0.08 & -0.00 & 0.04 & 0.04 & 0.07 & 0.08 & 0.08 \\
\hline 0.1 & -0.05 & -0.03 & -0.02 & 0.03 & 0.04 & 0.04 & 0.08 & 0.08 & 0.08 \\
\hline 0.05 & -0.02 & -0.02 & -0.01 & 0.04 & 0.04 & 0.04 & 0.08 & 0.08 & 0.08 \\
\hline 0 & -0.00 & -0.00 & -0.00 & 0.04 & 0.04 & 0.04 & 0.08 & 0.08 & 0.08 \\
\hline$C_{N E}$ & 0.25 & 0.4 & 0.5 & 0.25 & 0.4 & 0.5 & 0.25 & 0.4 & 0.5 \\
\hline$f$ & \multicolumn{3}{|c|}{$\overline{0.81}$} & \multicolumn{3}{|c|}{$\overline{0.9}$} & \multicolumn{3}{|c|}{$\overline{0.99}$} \\
\hline
\end{tabular}

Table 3.6: Figure 5: $\bar{C}_{B}$

\begin{tabular}{|c|c|c|c|c|c|c|c|c|c|}
\hline$\eta$ & \multicolumn{10}{|c|}{$C_{B}$} \\
\hline 1 & 0.51 & 0.56 & 0.60 & 0.33 & 0.35 & 0.37 & 0.25 & 0.26 & 0.26 \\
\hline 0.5 & 0.57 & 0.61 & 0.64 & 0.35 & 0.36 & 0.37 & 0.25 & 0.26 & 0.27 \\
\hline 0.1 & 0.71 & 0.70 & 0.70 & 0.37 & 0.38 & 0.38 & 0.26 & 0.27 & 0.27 \\
\hline 0.05 & 0.72 & 0.71 & 0.70 & 0.38 & 0.38 & 0.38 & 0.27 & 0.27 & 0.27 \\
\hline 0 & 0.74 & 0.74 & 0.74 & 0.38 & 0.37 & 0.39 & 0.27 & 0.27 & 0.27 \\
\hline \hline$C_{N E}$ & 0.25 & 0.4 & 0.5 & 0.25 & 0.4 & 0.5 & 0.25 & 0.4 & 0.5 \\
\hline \hline$f$ & \multicolumn{3}{|c|}{0.81} & \multicolumn{10}{c|}{0.9} & \multicolumn{5}{c|}{0.99} \\
\hline
\end{tabular}


Table 3.7: Figure 5: Reproductive probability

\begin{tabular}{|c|c|c|c|c|c|c|c|c|c|}
\hline$\eta$ & \multicolumn{9}{|c|}{ Reproductive probability (\%) } \\
\hline 1 & 1 & 11 & 22 & 41 & 78 & 82 & 77 & 83 & 84 \\
\hline 0.5 & 14 & 26 & 41 & 61 & 80 & 82 & 79 & 83 & 82 \\
\hline 0.1 & 59 & 66 & 71 & 77 & 81 & 82 & 82 & 83 & 83 \\
\hline 0.05 & 70 & 72 & 75 & 78 & 82 & 82 & 83 & 83 & 83 \\
\hline 0 & 79 & 79 & 79 & 82 & 82 & 82 & 83 & 83 & 83 \\
\hline$\overline{C_{N E}}$ & 0.25 & 0.4 & 0.5 & 0.25 & 0.4 & 0.5 & 0.25 & 0.4 & 0.5 \\
\hline 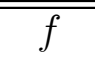 & & 0.81 & & & 0.9 & & & $\overline{0.99}$ & \\
\hline
\end{tabular}

Table 3.8: Figure 5: $\operatorname{Var}\left(C_{B}\right)$

\begin{tabular}{|c|c|c|c|c|c|c|c|c|c|}
\hline$\eta$ & \multicolumn{9}{|c|}{$\operatorname{Var}\left(C_{B}\right)$} \\
\hline 1 & .024 & .029 & .032 & .017 & .017 & .018 & .011 & .012 & .013 \\
\hline 0.5 & .029 & .034 & .036 & .018 & .018 & .019 & .012 & .013 & .014 \\
\hline 0.1 & .045 & .044 & .044 & .022 & .022 & .022 & .014 & .014 & .015 \\
\hline 0.05 & .051 & .047 & .047 & .022 & .023 & .024 & .014 & .014 & .015 \\
\hline 0 & .055 & .056 & .056 & .025 & .026 & .026 & .016 & .016 & .016 \\
\hline$\overline{\overline{C_{N E}}}$ & 0.25 & 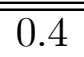 & 0.5 & 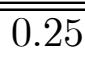 & 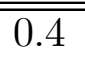 & 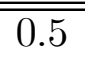 & 0.25 & 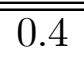 & 0.5 \\
\hline$\overline{f f}$ & \multicolumn{3}{|c|}{ 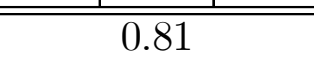 } & \multicolumn{3}{|c|}{$\overline{0.9}$} & \multicolumn{3}{|c|}{$\overline{0.99}$} \\
\hline
\end{tabular}




\subsubsection{Figure 3-6}

Table 3.9: Figure 6: Influence of maternal transfer on the population growth rate

\begin{tabular}{|c|c|c|c|c|c|c|c|c|c|}
\hline$\eta$ & \multicolumn{9}{|c|}{ Percent difference in population growth rates } \\
\hline 1 & -0.209 & -0.120 & -0.077 & -0.051 & 0.014 & 0.015 & 0.014 & 0.010 & 0.08 \\
\hline 0.5 & -0.118 & -0.083 & -0.050 & -0.006 & 0.016 & 0.012 & 0.012 & 0.009 & 0.003 \\
\hline 0.1 & -0.024 & -0.016 & -0.009 & -0.000 & 0.005 & 0.006 & 0.002 & 0.001 & 0.001 \\
\hline 0.05 & -0.011 & -0.007 & -0.004 & 0.001 & 0.002 & 0.004 & 0.002 & 0.003 & 0.001 \\
\hline 0 & 0.000 & -0.001 & -0.002 & 0.000 & -0.001 & -0.001 & -0.001 & -0.001 & -0.001 \\
\hline$\overline{\overline{C_{N E}}}$ & 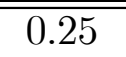 & $\overline{0.4}$ & "0.5 & $\overline{0.25}$ & $\overline{0.4}$ & 0.5 & 0.25 & $\overline{0.4}$ & 0.5 \\
\hline 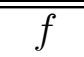 & \multicolumn{3}{|c|}{$\bar{~} 0.81$} & \multicolumn{3}{|c|}{$\overline{\overline{0.9}}$} & \multicolumn{3}{|c|}{ 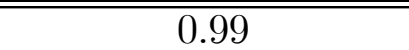 } \\
\hline
\end{tabular}

Table 3.10: Figure 6: Influence of the maternal transfer on $\bar{C}_{B}$

\begin{tabular}{|c|c|c|c|c|c|c|c|c|c|}
\hline$\eta$ & \multicolumn{10}{|c|}{ Percent difference in $\overline{C_{B}}$} \\
\hline 1 & 0.49 & 0.43 & 0.45 & 0.47 & 0.47 & 0.46 & 0.43 & 0.42 & 0.41 \\
\hline 0.5 & 0.50 & 0.48 & 0.47 & 0.47 & 0.44 & 0.45 & 0.42 & 0.41 & 0.41 \\
\hline 0.1 & 0.48 & 0.46 & 0.46 & 0.45 & 0.44 & 0.43 & 0.41 & 0.41 & 0.41 \\
\hline 0.05 & 0.46 & 0.44 & 0.44 & 0.43 & 0.44 & 0.42 & 0.41 & 0.40 & 0.40 \\
\hline 0 & 0.40 & 0.41 & 0.41 & 0.42 & 0.42 & 0.41 & 0.40 & 0.40 & 0.40 \\
\hline \hline$C_{N E}$ & 0.25 & 0.4 & 0.5 & 0.25 & 0.4 & 0.5 & 0.25 & 0.4 & 0.5 \\
\hline \hline$f$ & \multicolumn{3}{|c|}{0.81} & \multicolumn{7}{c|c}{0.9} & \multicolumn{7}{c|}{0.99} \\
\hline
\end{tabular}


Table 3.11: Figure 6: Influence of maternal transfer on the reproductive probability

\begin{tabular}{|c|c|c|c|c|c|c|c|c|c|}
\hline$\eta$ & \multicolumn{9}{|c|}{ Difference in reproductive probability $(\%)$} \\
\hline 1 & -70.3 & -60.1 & -52.5 & -31.3 & 2.4 & 1.4 & 2.2 & 0.7 & 0.3 \\
\hline 0.5 & -55.7 & -48.1 & -35.5 & -16.2 & 1.8 & 1.2 & 2.3 & 2.5 & 0.1 \\
\hline 0.1 & -16.0 & -11.5 & -6.7 & -2.1 & 1.2 & 1.0 & 0.1 & 0.4 & 0.8 \\
\hline 0.05 & -8.3 & -5.4 & -2.9 & -2.1 & 0.2 & 2.2 & 0.8 & -0.1 & -0.4 \\
\hline 0 & -0.4 & 0.2 & -1.0 & 0.3 & 1.7 & 0.1 & -0.6 & -0.6 & -0.6 \\
\hline \hline$C_{N E}$ & 0.25 & 0.4 & 0.5 & 0.25 & 0.4 & 0.5 & 0.25 & 0.4 & 0.5 \\
\hline \hline$f$ & \multicolumn{3}{|c|}{0.81} & \multicolumn{3}{c|}{0.9} & \multicolumn{5}{c|}{0.99} \\
\hline
\end{tabular}

Table 3.12: Figure 6: Influence of maternal transfer on the variance of $C_{B}$

\begin{tabular}{|c|c|c|c|c|c|c|c|c|c|}
\hline$\eta$ & \multicolumn{10}{|c|}{ Difference in $\operatorname{Var}\left(C_{B}\right)$} \\
\hline 1 & -0.01 & -0.03 & -0.04 & -0.02 & -0.03 & -0.04 & -0.01 & -0.01 & -0.01 \\
\hline 0.5 & -0.04 & -0.04 & -0.06 & -0.03 & -0.04 & -0.04 & -0.01 & -0.01 & -0.01 \\
\hline 0.1 & -0.11 & -0.12 & -0.12 & -0.05 & -0.06 & -0.06 & -0.02 & -0.02 & -0.02 \\
\hline 0.05 & -0.13 & -0.15 & -0.16 & -0.07 & -0.07 & -0.07 & -0.02 & -0.02 & -0.02 \\
\hline 0 & -0.23 & -0.23 & -0.23 & -0.09 & -0.08 & -0.09 & -0.02 & -0.02 & -0.02 \\
\hline \hline$C_{N E}$ & 0.25 & 0.4 & 0.5 & 0.25 & 0.4 & 0.5 & 0.25 & 0.4 & 0.5 \\
\hline \hline$f$ & \multicolumn{10}{|c|}{0.81} & \multicolumn{8}{c|c}{0.9} & \multicolumn{5}{c|}{0.99} \\
\hline
\end{tabular}




\subsubsection{Figure 3-9}

Table 3.13: Figure 9: Asymptotic $C_{B}$.

\begin{tabular}{|l||c|c|c|}
\hline \multicolumn{4}{|c|}{ Asymptotic $\overline{C_{B}}$} \\
\hline Mode $`^{f}$ & 0.81 & 0.90 & 0.99 \\
\hline \hline M-C+ + & 0.70 & 0.38 & 0.27 \\
\hline M-C0 & 0.30 & 0.17 & 0.11 \\
\hline M0C0 & 0.48 & 0.25 & 0.16 \\
\hline
\end{tabular}

Table 3.14: Figure 9: Introduction rate of the toxicant into the population.

\begin{tabular}{|l||l|l|l|}
\hline \multicolumn{4}{|c|}{ Assimilation rate $\left(\Gamma_{I}\right)$} \\
\hline Mode $\backslash^{f}$ & 0.81 & 0.90 & 0.99 \\
\hline \hline $\mathrm{M}-\mathrm{C}+$ & 0.036 & 0.064 & 0.084 \\
\hline $\mathrm{M}-\mathrm{C} 0$ & 0.074 & 0.110 & 0.171 \\
\hline $\mathrm{M} 0 \mathrm{C} 0$ & 0.056 & 0.090 & 0.128 \\
\hline
\end{tabular}

Table 3.15: Figure 9: Depuration rate of the toxicant from the population.

\begin{tabular}{|l||l|l|l|}
\hline \multicolumn{4}{|c|}{ Depuration rate $\left(\Gamma_{D}\right)$} \\
\hline Mode $\backslash^{f}$ & 0.81 & 0.90 & 0.99 \\
\hline \hline $\mathrm{M}-\mathrm{C}+$ & 0.031 & 0.062 & 0.087 \\
\hline $\mathrm{M}-\mathrm{C} 0$ & 0.084 & 0.127 & 0.154 \\
\hline $\mathrm{M} 0 \mathrm{C} 0$ & 0.062 & 0.093 & 0.124 \\
\hline
\end{tabular}




\subsection{Appendix B : Model code}

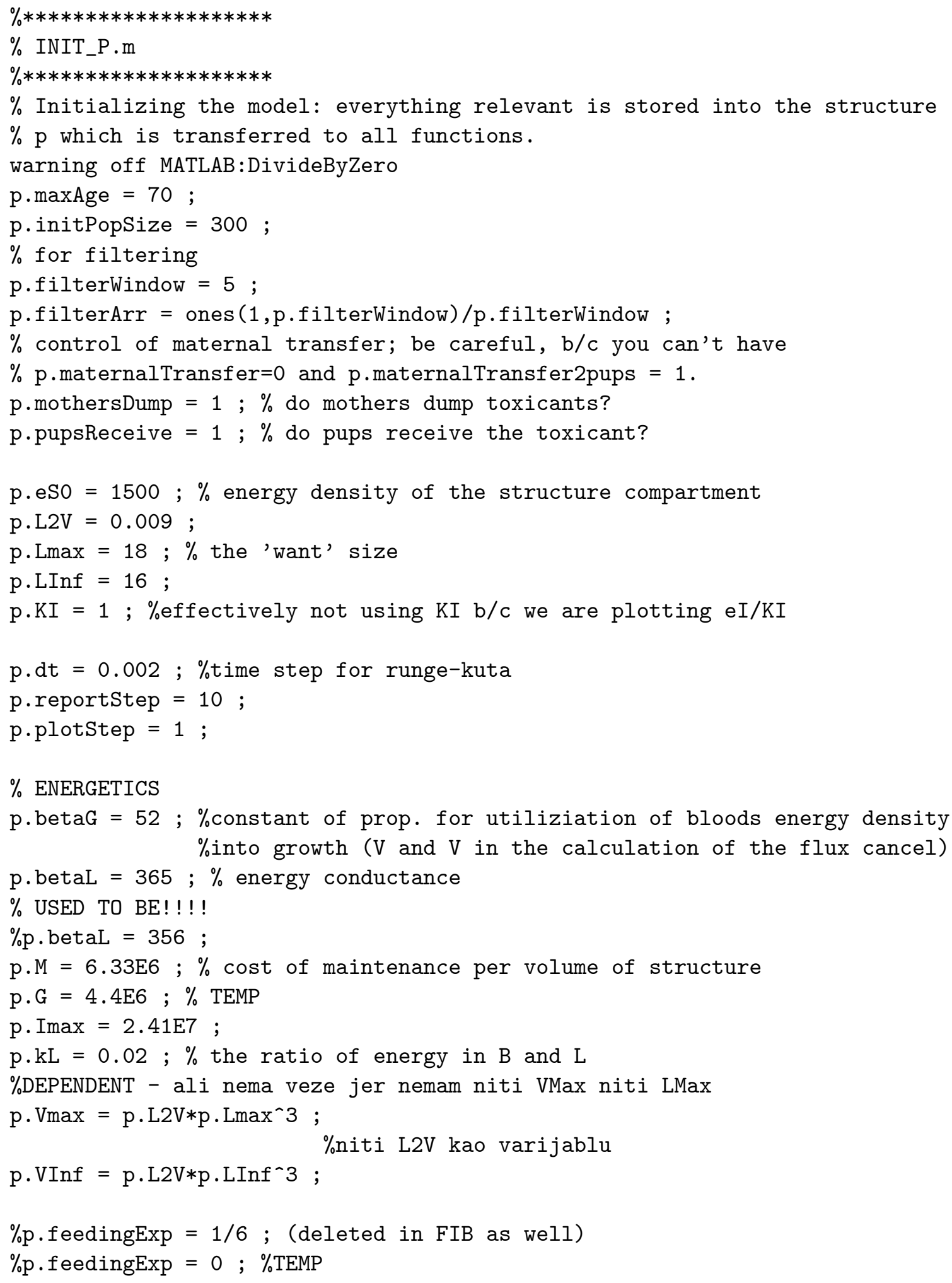




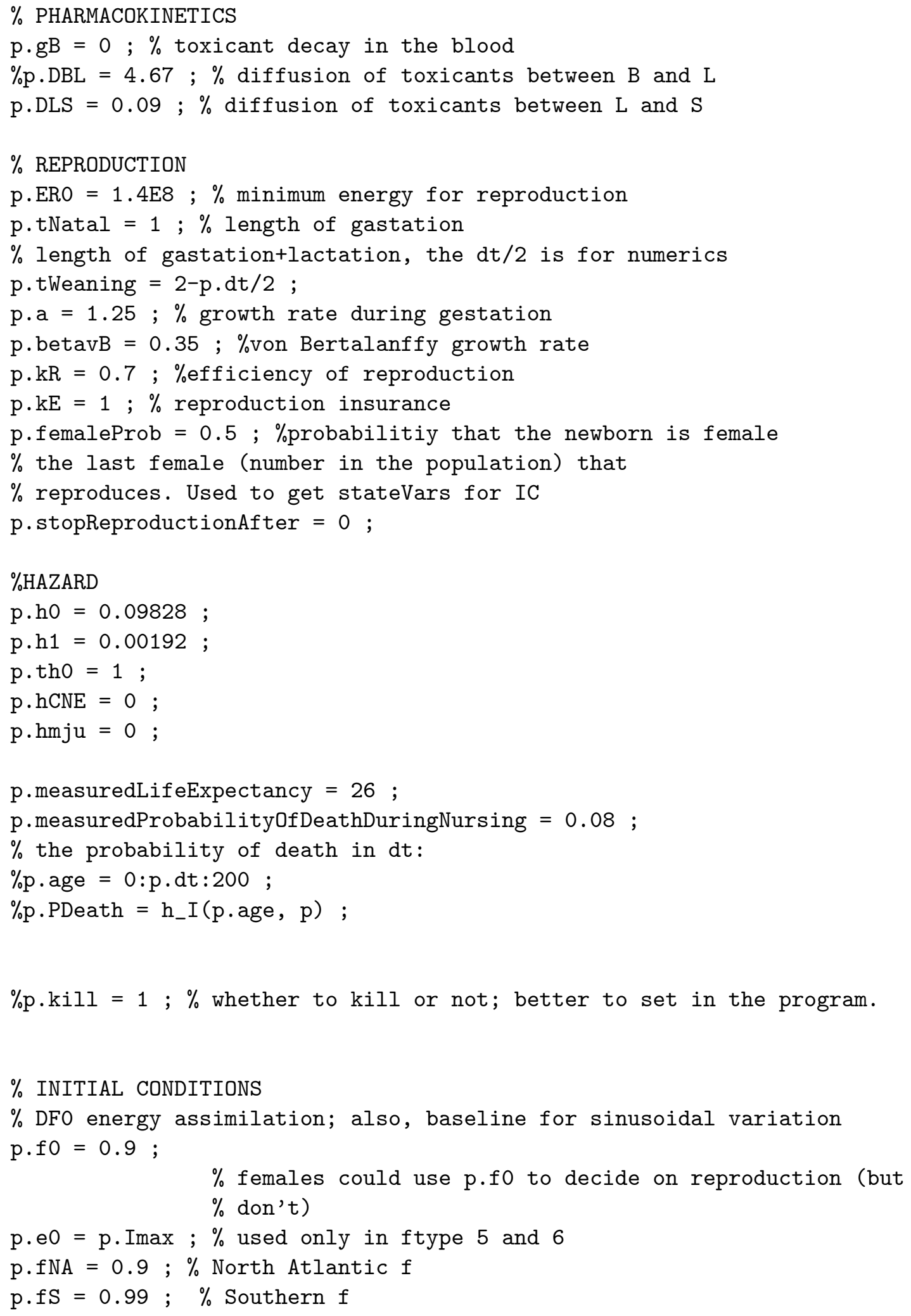




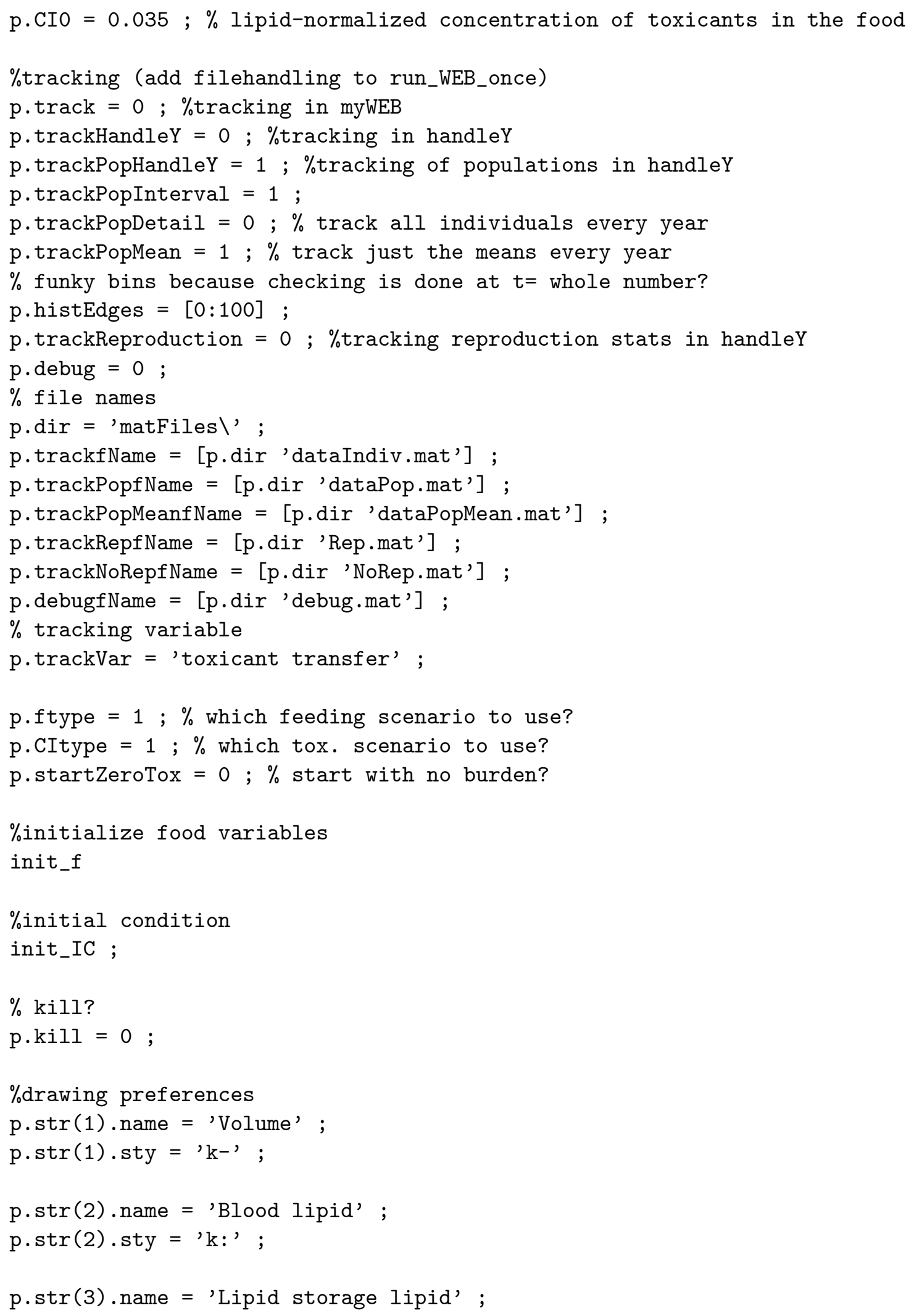




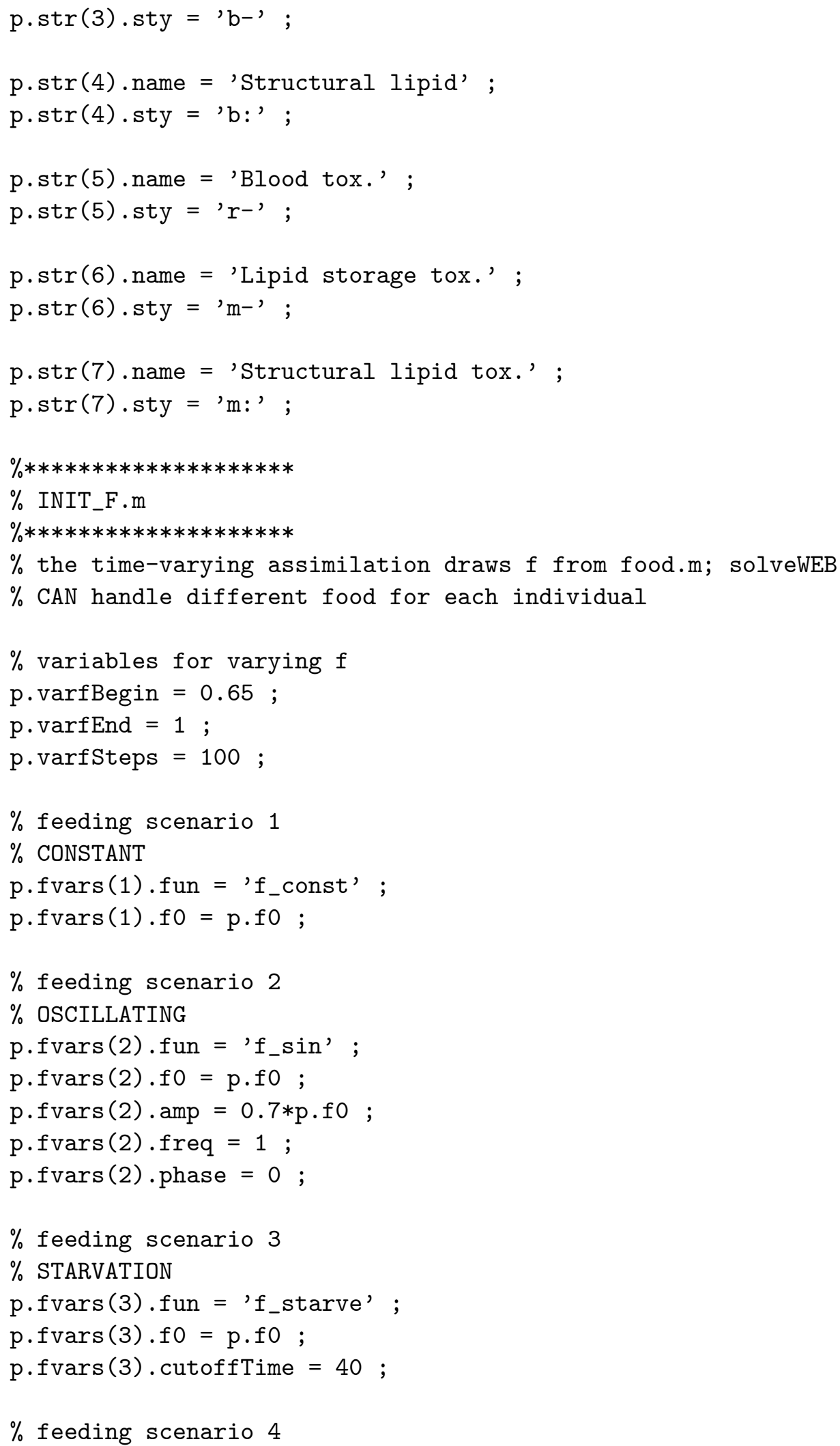




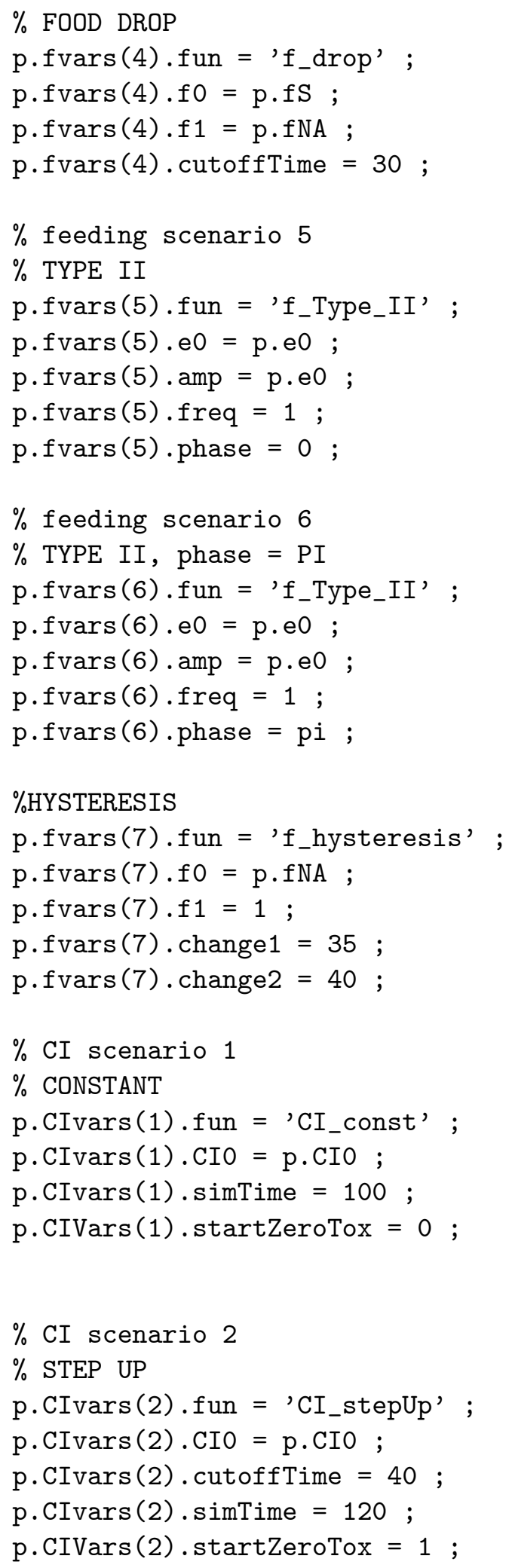




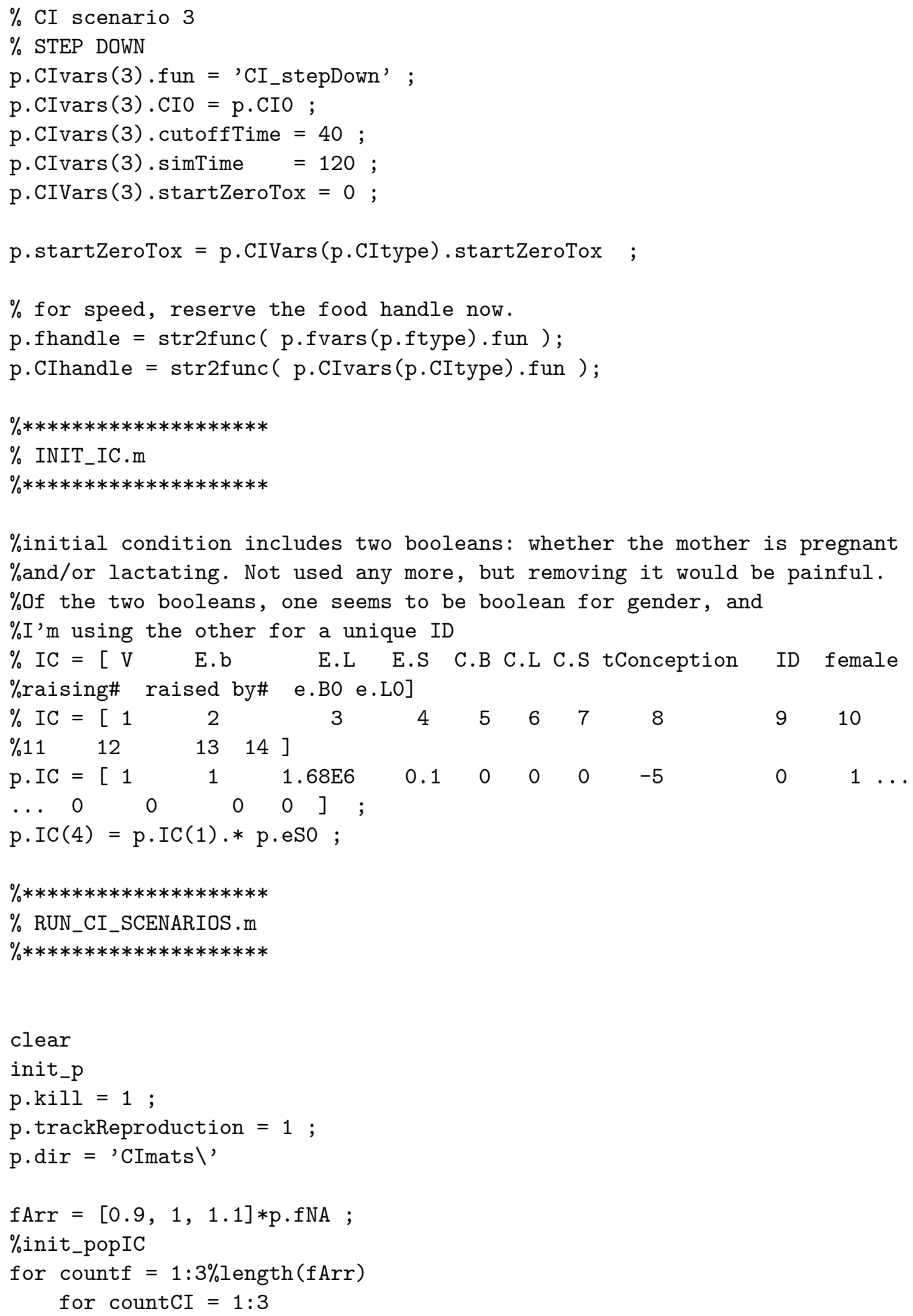




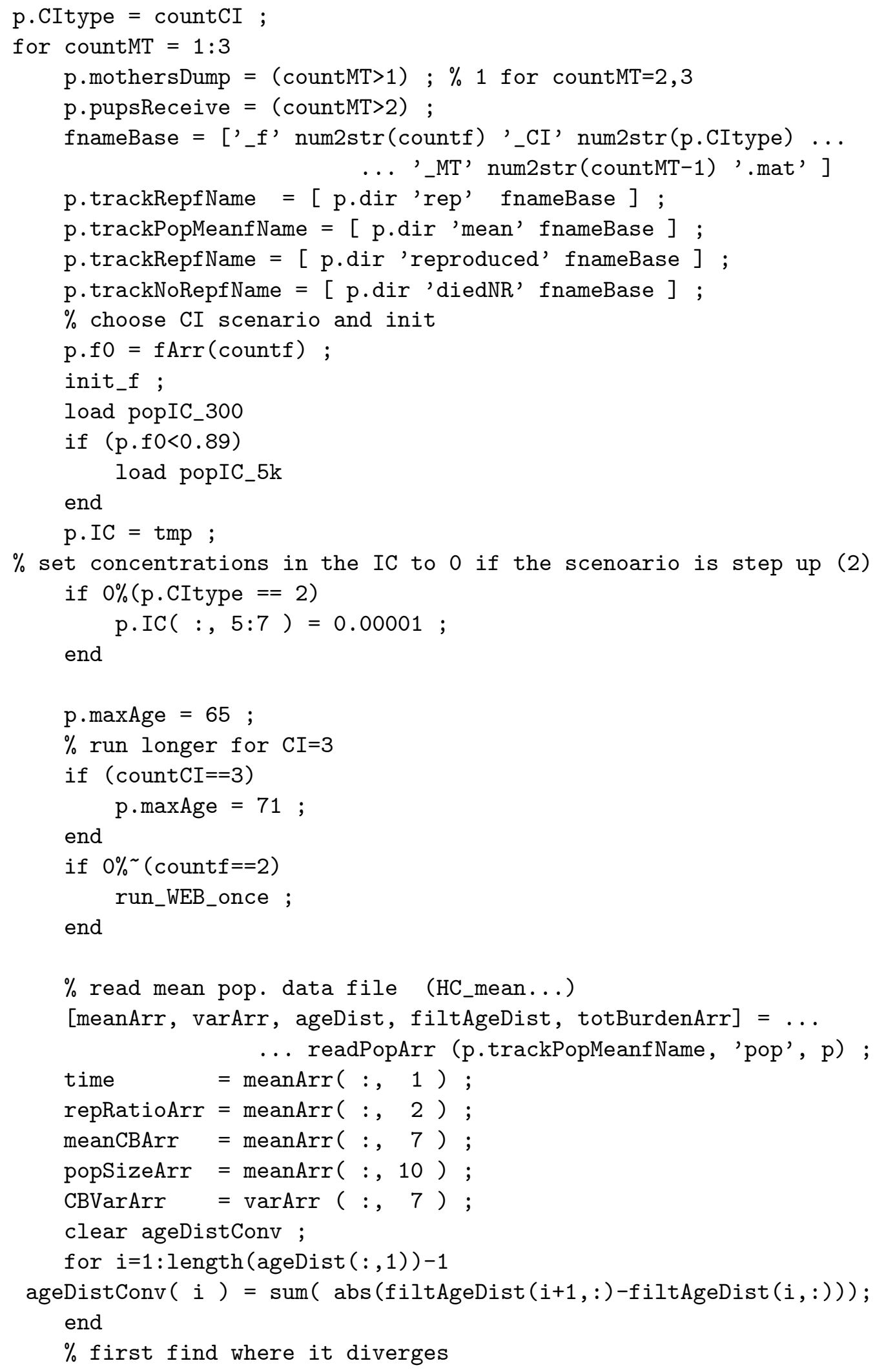




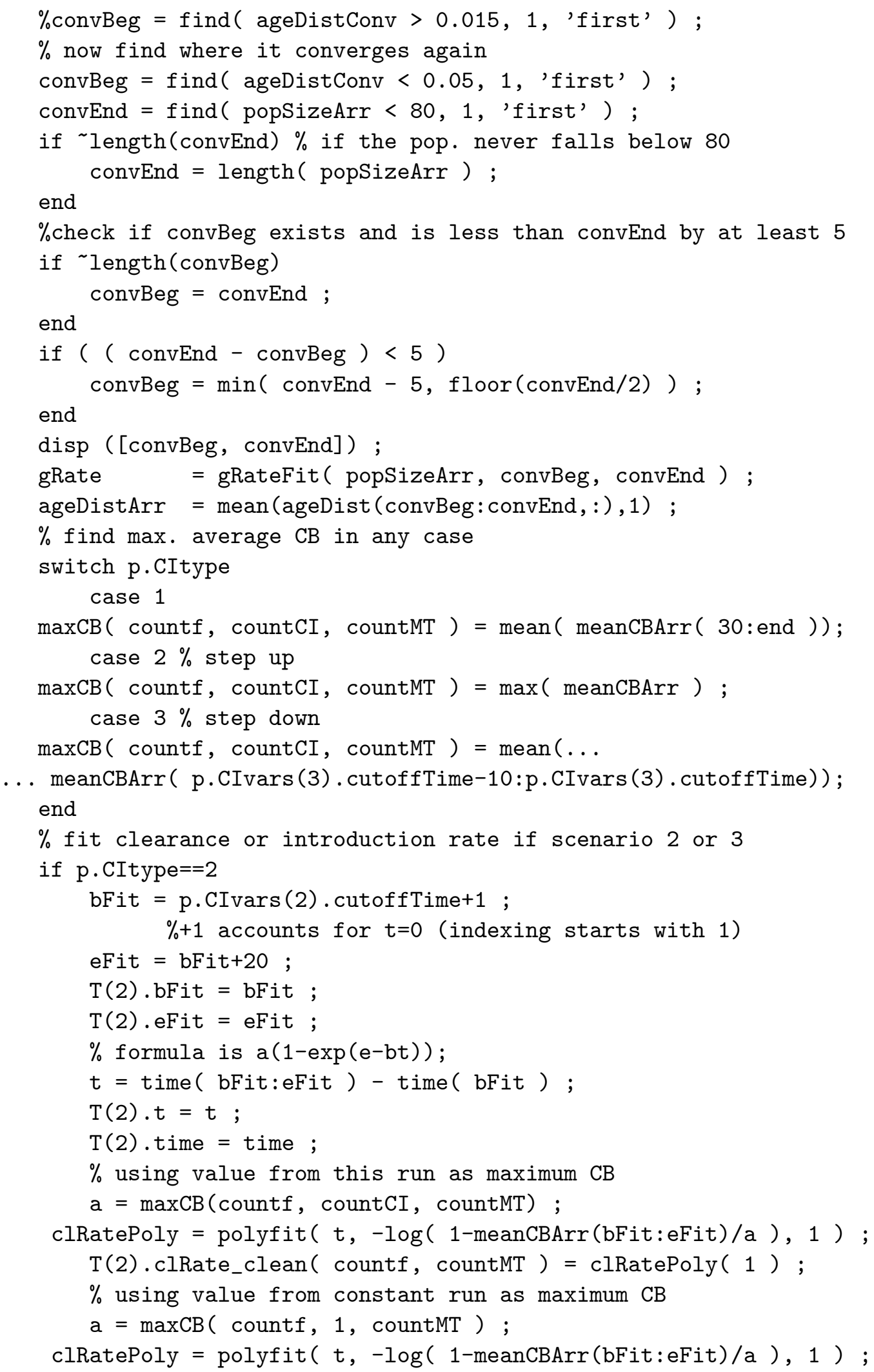




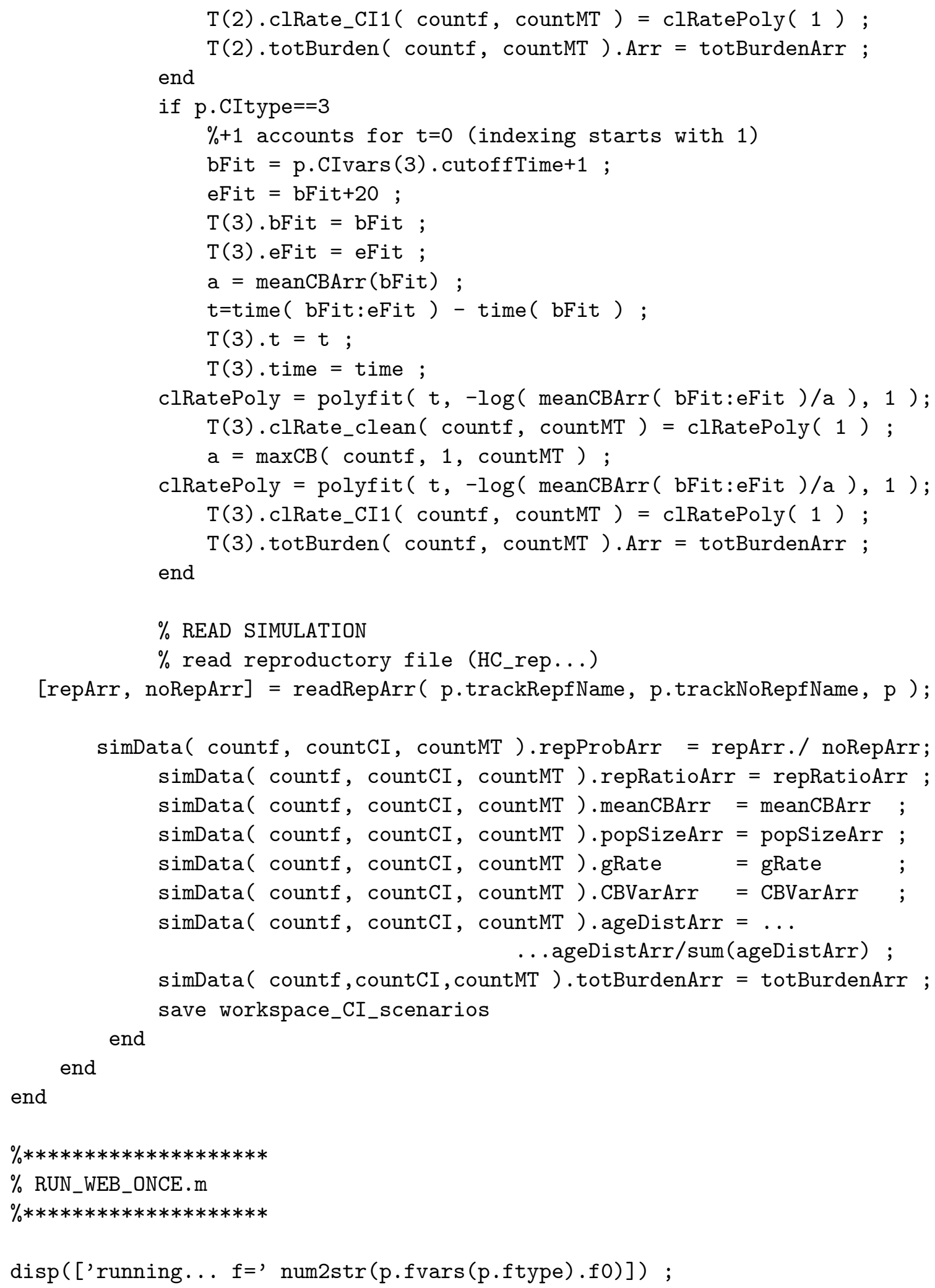




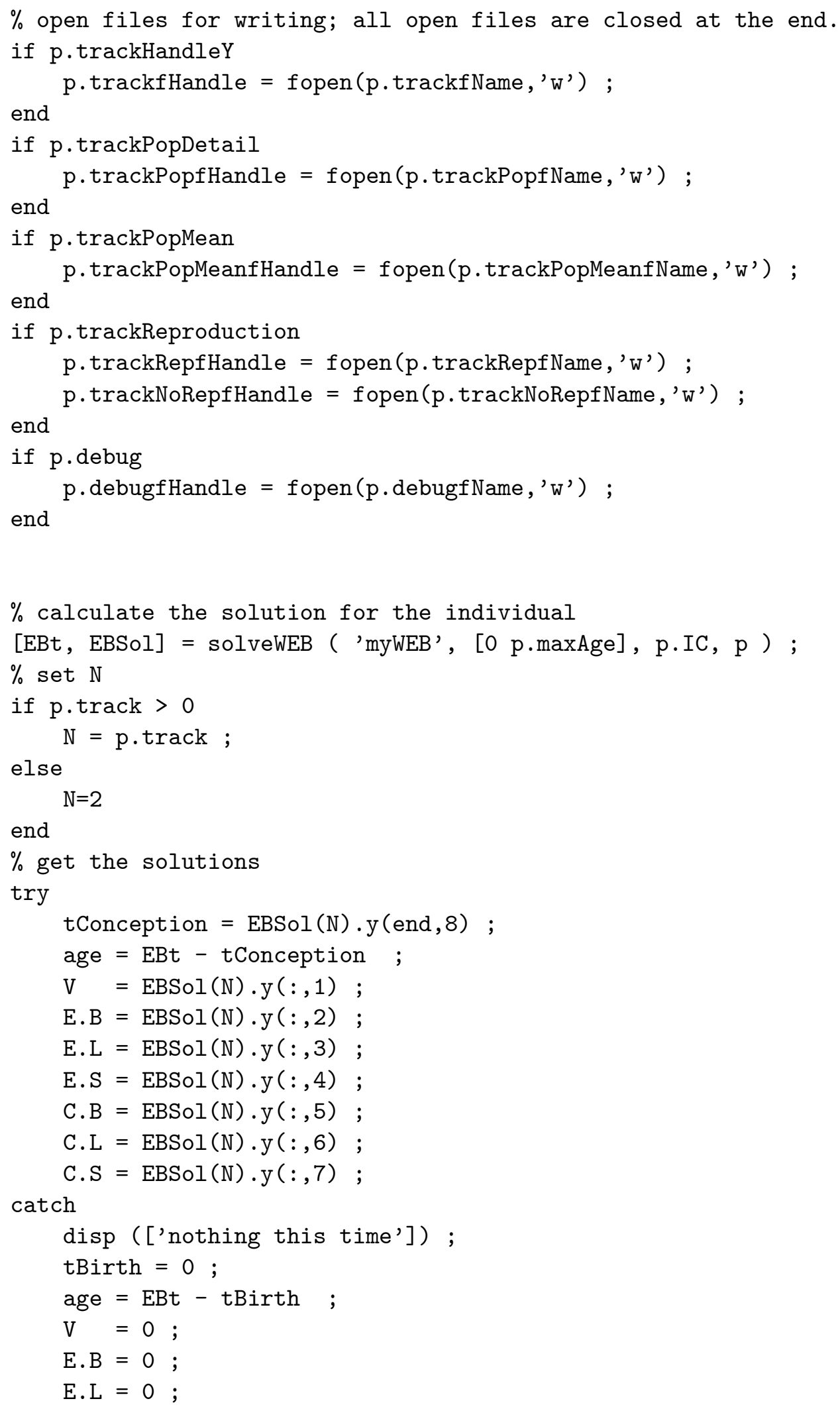




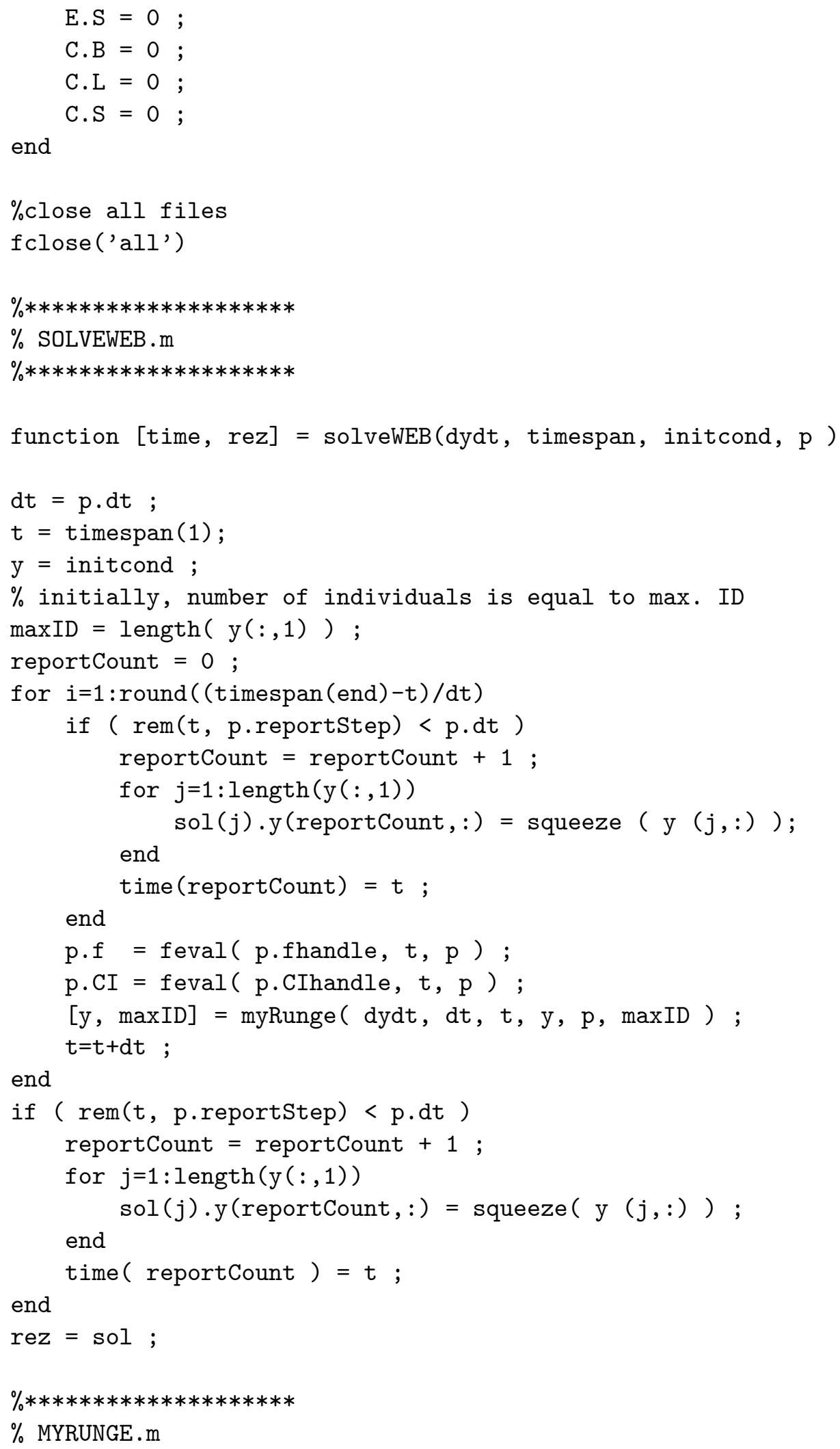




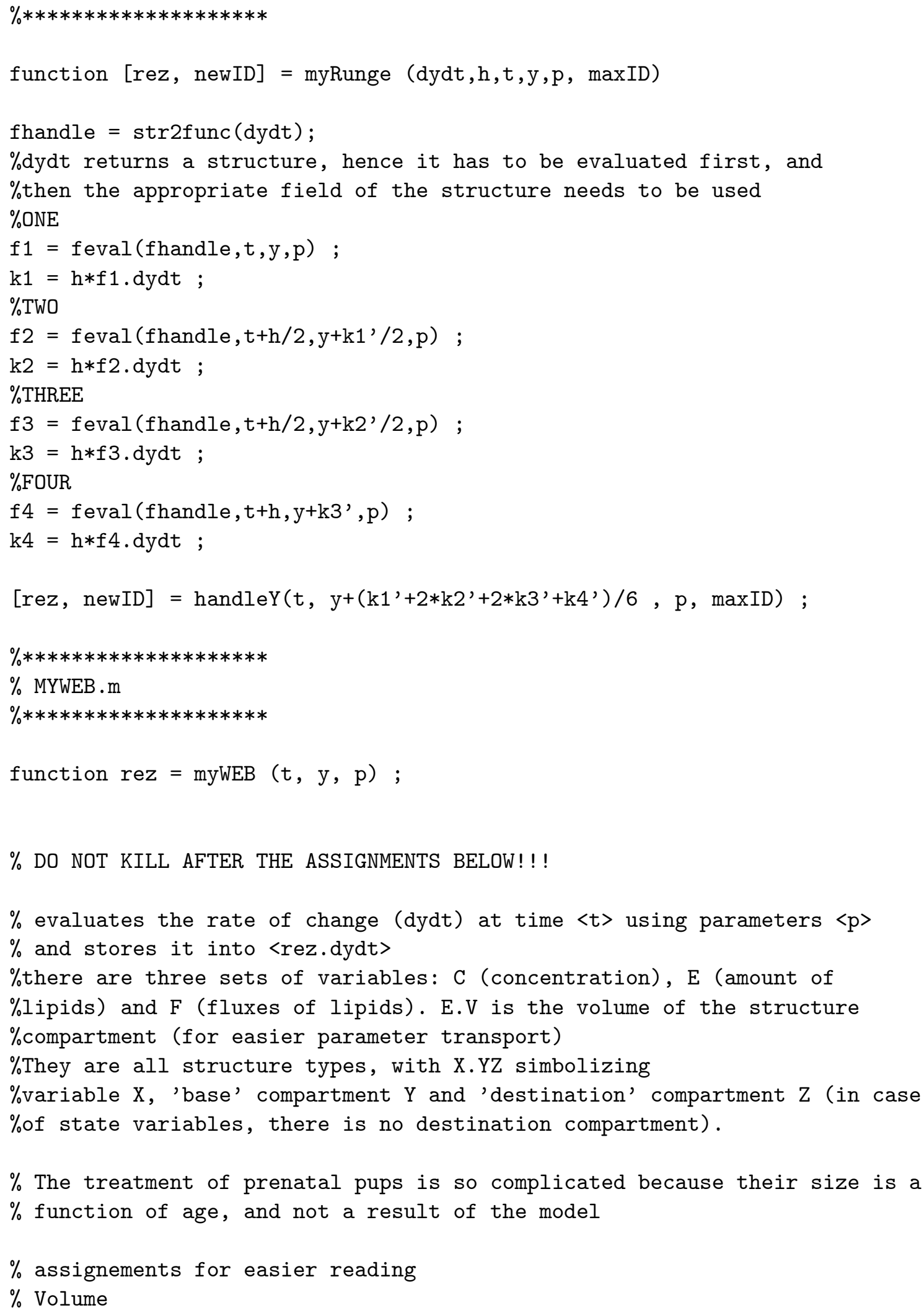




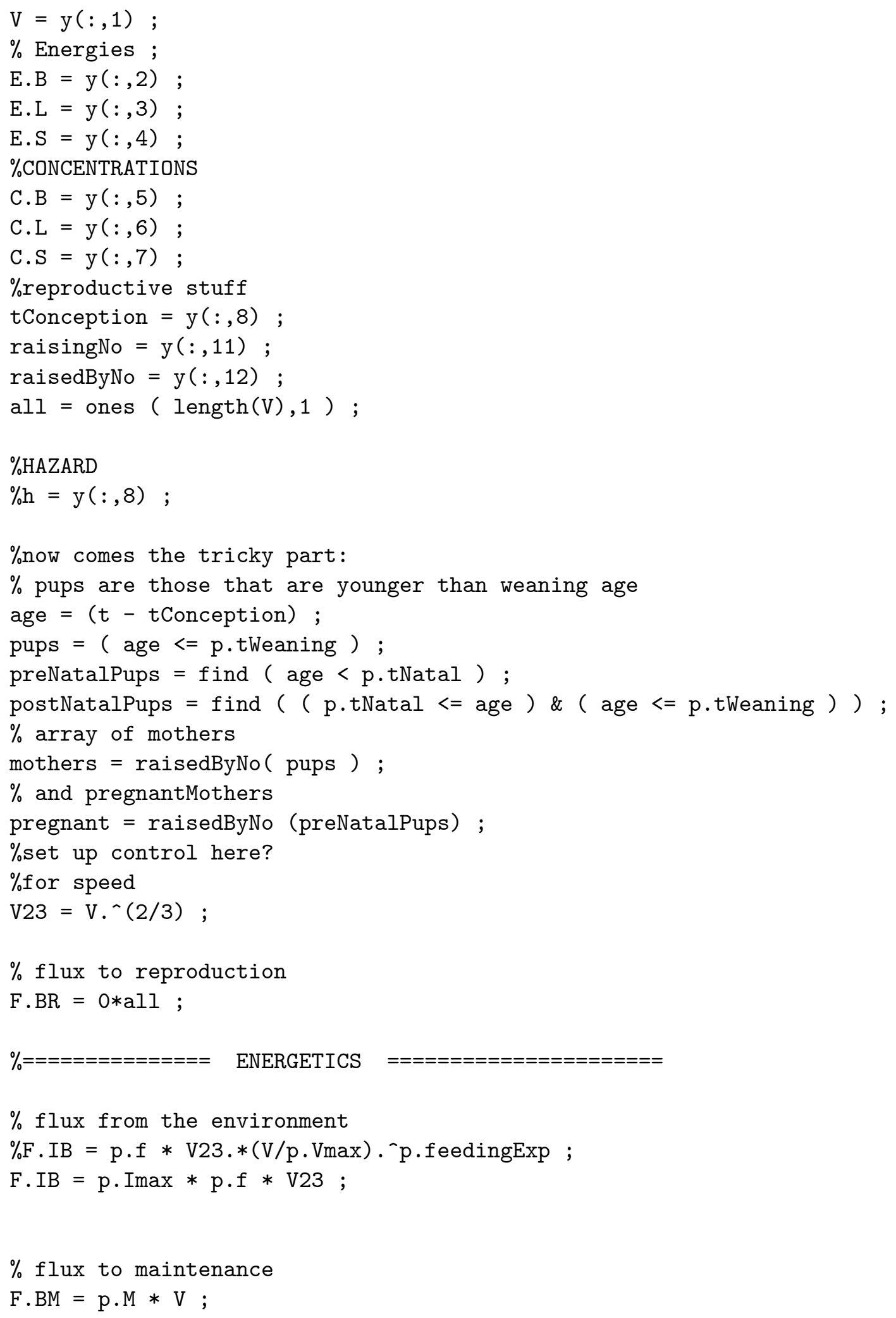


$\%$ flux to growth (note that, if times are good, growth continues even when $\%$ the animal is reproducing

$\mathrm{F} \cdot \mathrm{BG}=\max (0,(\mathrm{p} \cdot \operatorname{betaG} * \mathrm{E} \cdot \mathrm{B}-\mathrm{F} \cdot \mathrm{BM}))$;

$\%$ exchange of lipids between the blood and lipid storage compartment

$\%$ Flux between $B$ and L, positive for the flow FROM B TO L

$\mathrm{F} . \mathrm{BL}=$ p.betaL* E.B ;

$F \cdot L B=p \cdot b e t a L * p \cdot k L * E \cdot L$;

$\%$ PRE-NATAL PUPS $1 / 3$

$\%$ rate of change of size

prNdVdt = prenataldVdt ( age (preNatalPups), p ) ;

$\%$ cost of increasing the blood and storage lipids to meet the maintenance

$\%$ (see manuscript)

prN.FRB $=$ p.M $*$ prNdVdt/p.betaL $; \%$ dE^\{Fetus)_B $/ d t$

prN.FRL $=$ prN.FRB/p.kL ; \% dE^\{Fetus)_L/dt

$\%$ cost of growth

F.BG (preNatalPups) = p.G $*$ prNdVdt ;

$\%$ EVERYBODY

\%growth

dydt.V = F.BG / p.G ;

$\%$ flux to structural lipids

F.LS $=$ p.eSO $*$ dydt.V ;

$\%$ PRENATAL PUPS $2 / 3$

$\%$ energy needed = energy for growth + maintenance

F.IB ( preNatalPups $)=\operatorname{prN} . \mathrm{FRB}+\ldots$

$\ldots+$ prN.FRL + F.BG(preNatalPups) + F.LS(preNatalPups) + F.BM(preNatalPups);

$\%$ POSTNATAL PUPS

$\%$ POSSIBLE

$\mathrm{F} \cdot \mathrm{BRMax}=\max (0, \mathrm{E} \cdot \mathrm{B} / \mathrm{p} \cdot \mathrm{dt}-(\mathrm{F} \cdot \mathrm{IB}+(\mathrm{F} \cdot \mathrm{LB}-\mathrm{F} \cdot \mathrm{BL})-\mathrm{F} \cdot \mathrm{BM}-\mathrm{F} \cdot \mathrm{BG}))$;

$\%$ ideal energy from mother during nursing

$\%$ target energy densities

e.BO $=\mathrm{y}(:, 13)$;

e.LO = y $(:, 14)$

e.SO = p.eSO ;

F.IBIdeal $=$ postNatal $($ age $, p, e) ;$

F.IB (postNatalPups) $=\min ($ F.IBIdeal (postNatalPups), $\ldots$ ...p.kR*F.BRMax ( raisedByNo( postNatalPups ) ) ) ;

$\%$ EVERYBODY

$\%$ rate of change of structural lipids 
dydt.E.S = F.LS ;

$\%$ rate of change of the lipid storage compartment

dydt.E.L = F.BL - F.LB - F.LS ;

$\%$ the energy output in mothers is

try

F.BR ( raisedByNo ( pups ) ) $=\mathrm{F} \cdot \mathrm{IB}($ pups $) / \mathrm{p} \cdot \mathrm{kR}$;

catch

keyboard

end

$\%$ rate of change of lipids in the blood

dydt.E.B = F.IB + (F.LB - F.BL $)-F \cdot B R-F . B M-F . B G$;

$\%$ PRENATAL PUPS $3 / 3$

$\%$ growth and rate of change of struct. lipids as for everybody

$\%$ the two lipid compartments increase proportionaly to $\mathrm{V}$;

$\% \mathrm{~B}$ and $\mathrm{L}$ do not exchange anything during gestation.

$\%$ rate of change of the lipid storage compartment

dydt.E.L(preNatalPups) = prN.FRL;

$\%$ rate of change of lipids in the blood

dydt.E.B(preNatalPups) = prN.FRB ;

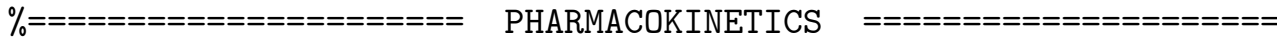

$\%$ mothers are receiving environemtnal concentration

toxIntake $=\mathrm{p} . \mathrm{CI} . * \mathrm{~F} . \mathrm{IB}$;

$\%$ for toxicological purposes, maintenance and growth of fetuses does

$\%$ not increase their C.B (it gets washed away by the mother)

$\%$ but it does increase their mothers' $C B$

F.BM( pregnant ) =F.BM (pregnant) $+F . B M($ preNatalPups) $+F . B G$ (preNatalPups);

$\%$ and it does not count as a part of $\mathrm{F} . \mathrm{BR}$

F.BR (pregnant) $=F . B R$ (pregnant) $-F . B M($ preNatalPups) $-F . B G$ (preNatalPups);

$\%$ I could just set concentrations for fetuses to their mothers'C.B,

$\%$ but that would mean mass balances troubles when mothers' C.B change.

$\%$ for pups

toxIntake( pups ) = C.B ( mothers ).* F.IB( pups )*p.pupsReceive ;

$\%$ check that pups are not reproducing

if $\operatorname{sum}(\mathrm{F} . \mathrm{BR}(\mathrm{pups})>0$ )

keyboard

end

$\%$ toxicant diffusion between $\mathrm{L}$ and $\mathrm{S}$

DLS $=$ p.DLS. $*($ C.L - C.S $) . *$ V23 ; 
$\%$ rate of change of toxicant concentration in blood (B). F.BL is a sink if $\% \mathrm{~F} . \mathrm{BL}>0$, and source if $\mathrm{F} . \mathrm{BL}<0$. Also, if there is no energy, concentration=0 dydt.C.B $=($ toxIntake $+($ C.L.*F.LB - C.B.*F.BL $)-\ldots$ ..C.B.* F.BR*p.mothersDump - C.B.*dydt.E.B )./ E.B - C.B.*p.gB ; $\%$ only what actually stays in the pup contributes to its toxicant burden dydt.C.B ( preNatalPups $)=($ prN.FRB .* ( C.B ( pregnant ) - ... ...C.B( preNatalPups ) ) )./ E.B( preNatalPups ) ;

if $(\operatorname{sum}(E \cdot B==0))$

$\%$ used to check that clipping only occurs until age $>0.2$ clipping $=$ find $(E \cdot B==0)$; dydt.C.B (clipping) $=0$;

end

$\%$ rate of change of toxicant concentration in lipid storage (L) dydt.C.L = ( (C.B.*F.BL - C.L.*F.LB) - F.LS - C.L.*dydt.E.L )./ E.L - DLS; dydt.C.L ( preNatalPups $)=\ldots$

...(prN.FRL .*(C.B(pregnant) -C.L(preNatalPups)))./E.L(preNatalPups ) ; dydt.C.L $(E . L==0)=0$;

$\%$ rate of change of toxicant concentration in structural lipids (S)

$\%$ for prenatal pups I make a mistake of assuming the structural lipids come $\%$ directly from the mother... otherwise it gets really complicated $\%$ because I'd have to balance all fluxes (to and from all compartments), $\%$ which is very tricky when volume is a function of age. dydt.C.S = ( C.L.*F.LS - C.S.*dydt.E.S )./ E.S + DLS ; dydt.C.S ( preNatalPups $)=($ F.LS ( preNatalPups ) .* ... ...( C.B ( pregnant ) - C.S ( preNatalPups ) ) )./ E.S( preNatalPups ); dydt.C.S $(E . S==0)=0$;

if 0

try $\% \operatorname{disp}\left(\left[{ }^{\prime} \mathrm{C} . \mathrm{B}={ }^{\prime}\right.\right.$ num2str(C.B(2)) ', $\mathrm{E} . \mathrm{B}={ }^{\prime}$ num2str(E.B(2))]) ;

if $\sim(E . B(2)==0)$ keyboard

end if ( C. B (2)==0) keyboard

end

if isnan(C.B(2)) keyboard

end

end

end

$\%$ make sure prenatals are not changing 


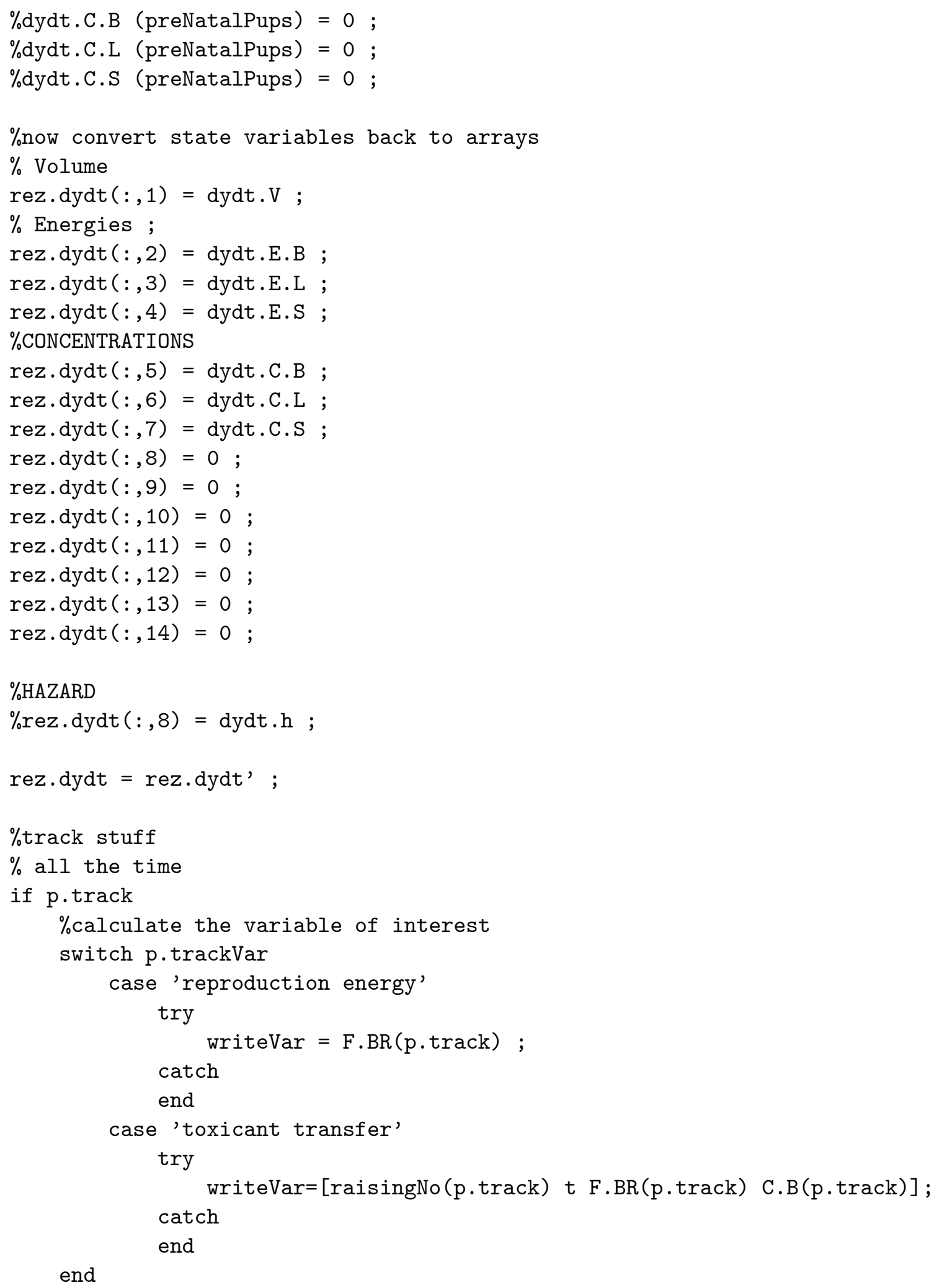




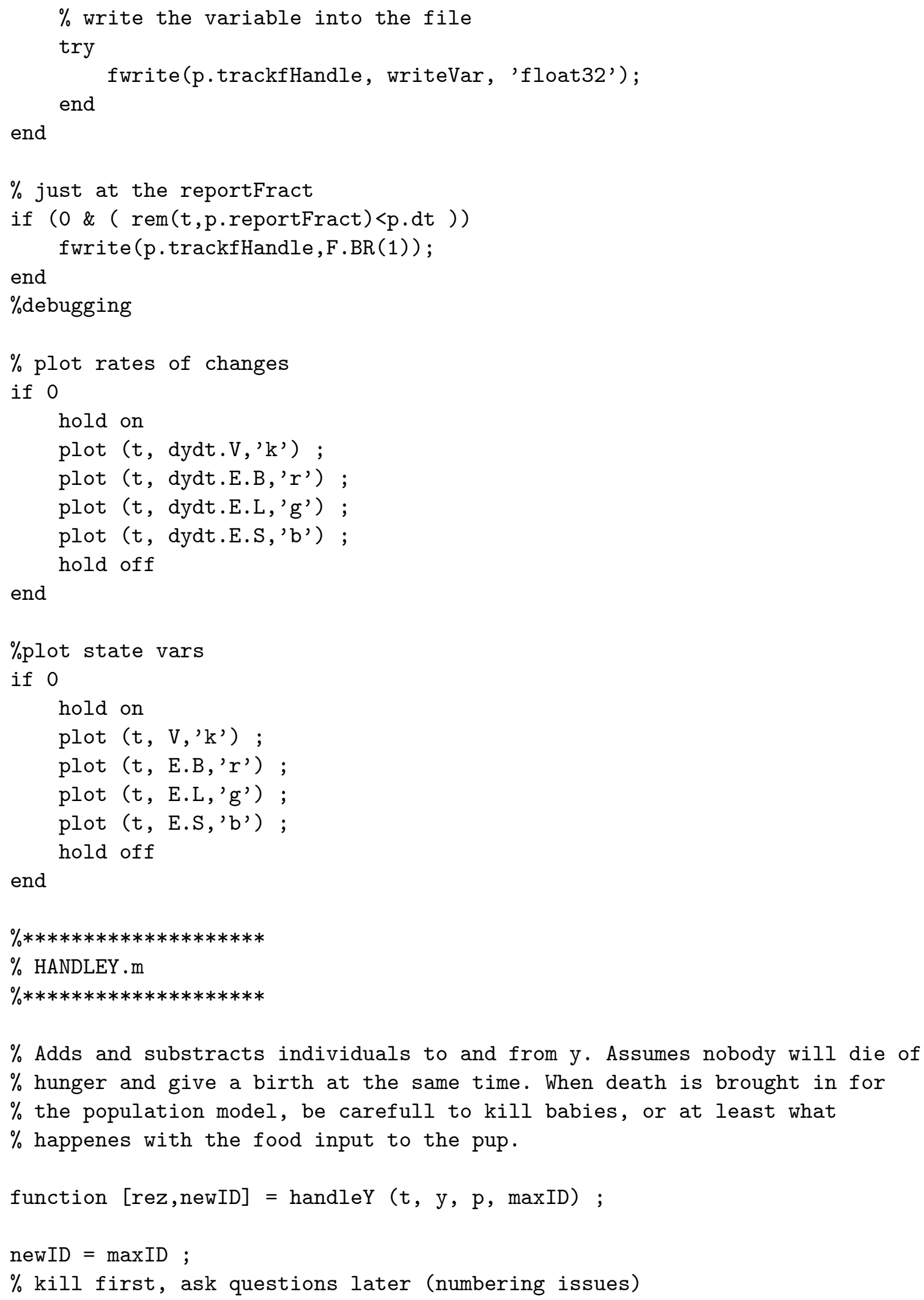

$\%$ Adds and substracts individuals to and from y. Assumes nobody will die of $\%$ hunger and give a birth at the same time. When death is brought in for $\%$ the population model, be carefull to kill babies, or at least what $\%$ happenes with the food input to the pup.

function $[r e z, n e w I D]=$ handleY $(t, y, p, \operatorname{maxID})$;

newID $=\operatorname{maxID} ;$

$\%$ kill first, ask questions later (numbering issues) 
$\%$ but first...

$\%$ pups are those that are younger than weaning age

tConception $=\mathrm{y}(:, 8)$;

raisingNo $=\mathrm{y}(:, 11) ; \%$ not reset after weaning

raisedByNo $=y(:, 12) ;$

$\%$ pups are those that are younger than weaning age

age $=t-$ tConception;

pups $=($ age $<$ p.tWeaning $) ;$

$\%$ array of mothers

$\%$ use only mothers, as raisingNo is not reset after weaning

mothers = raisedByNo( pups ) ;

$\%$ now determine who died

$\%$ The lipids in the blood or in the energy storage (E.B or E.L) cannot

$\%$ be negative. kill those that have negative E.B or L.B or had bad luck.

if p.kill

condemned $=\operatorname{find}((y(:, 2)<0)|(y(:, 3)<0)| \operatorname{died}(\operatorname{age}, y(:, 5), p))$;

else

condemned $=[]$;

end

$\%$ determine which pups go because their mother is done for

$\%$ you do not need to worry about moms whose pup died - their FBR

$\%$ should be 0 , as all relations are determined by the pups

$\%(y(:, 9: 10)$ are unneccessary (?)).

try

if length(condemned)

pupsKilled = raisingNo $($ intersect $($ condemned, mothers $)$ ) ;

condemned $=$ [condemned; pupsKilled ];

$\% \operatorname{disp}([' t=$ ' num2str( $t)$ '; ' num2str(length(condemned)) ' die: '... ...num2str ( condemned') ' ID ' num2str ( y( condemned, 9 )' )]) ;

end

$\%$ take out possible duplicates

condemned $=$ unique ( condemned ) ;

$\%$ adjust mother-pup links

for $i=1$ : length (condemned)

tmpi = condemned $(i)$;

adjustRaisingNo $=$ find ( raisingNo $>$ tmpi ) ;

adjustRaisedByNo $=$ find $($ raisedByNo $>$ tmpi $)$;

$\mathrm{y}($ adjustRaisingNo, 11) = y (adjustRaisingNo, 11) - 1 ;

$\mathrm{y}($ adjustRaisedByNo, 12) = y(adjustRaisedByNo, 12) - 1 ;

$\%$ capture individuals that have not reproduced but were born

if (p.trackReproduction* $(\tilde{y}(\operatorname{tmpi}, 11)) *((t-y(t m p i, 8))<=1))$

try

$\%$ write time, time of conception, ID, volume 


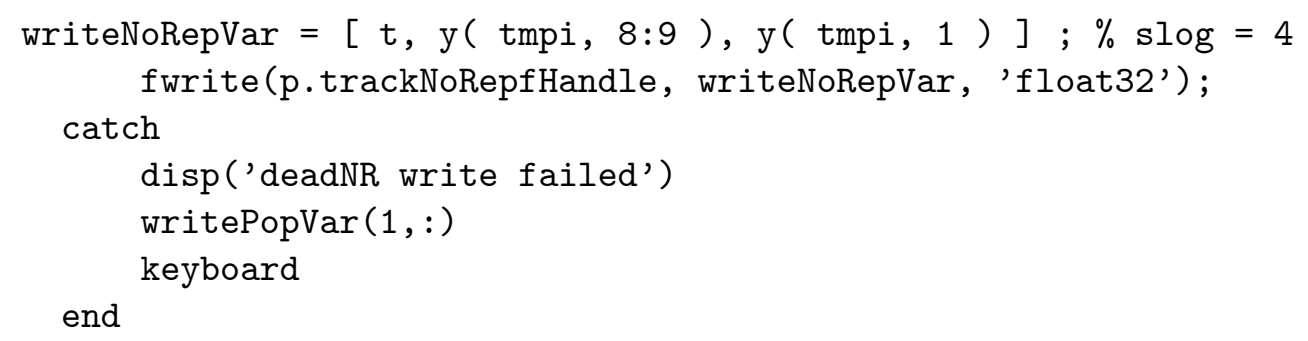




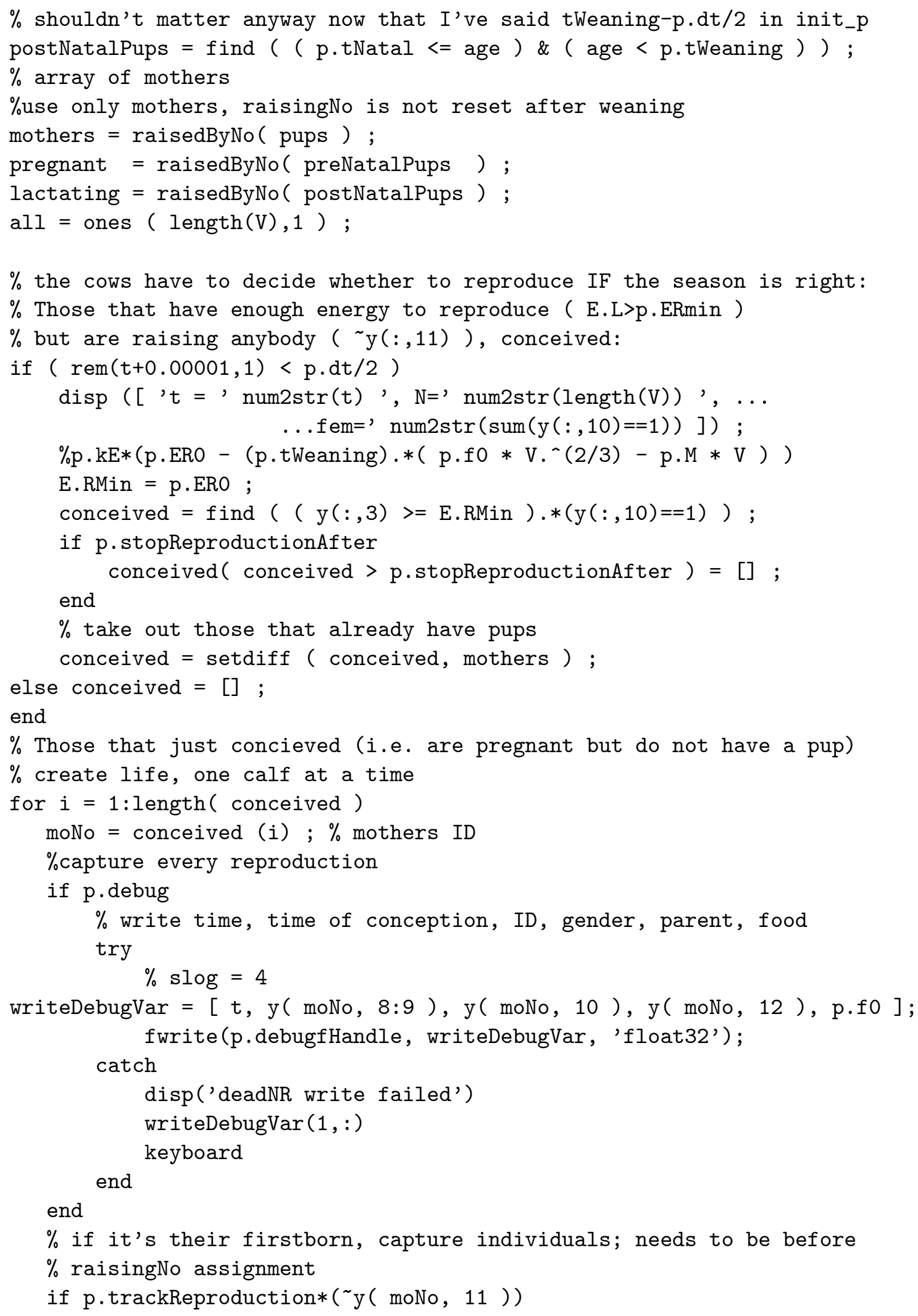




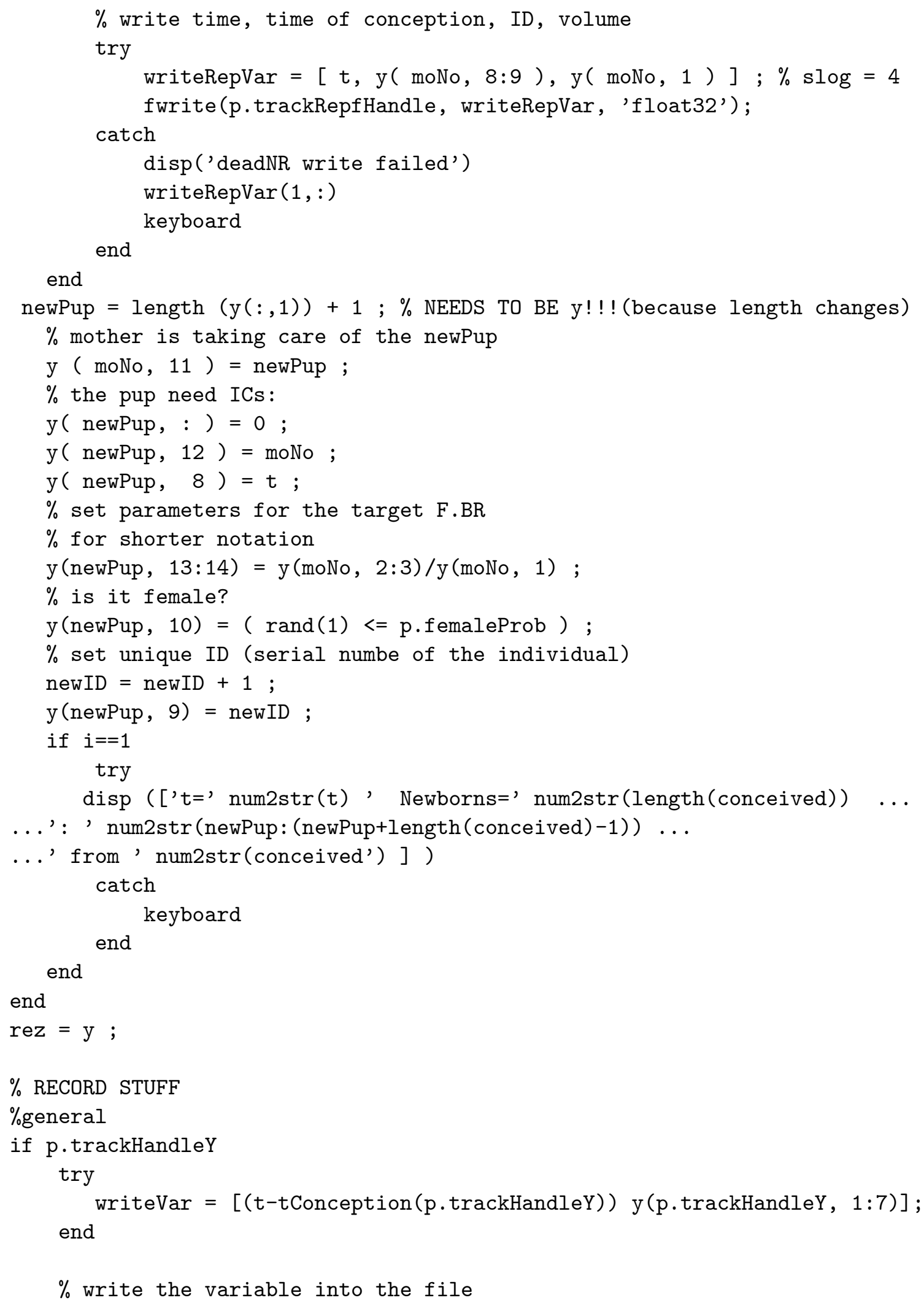




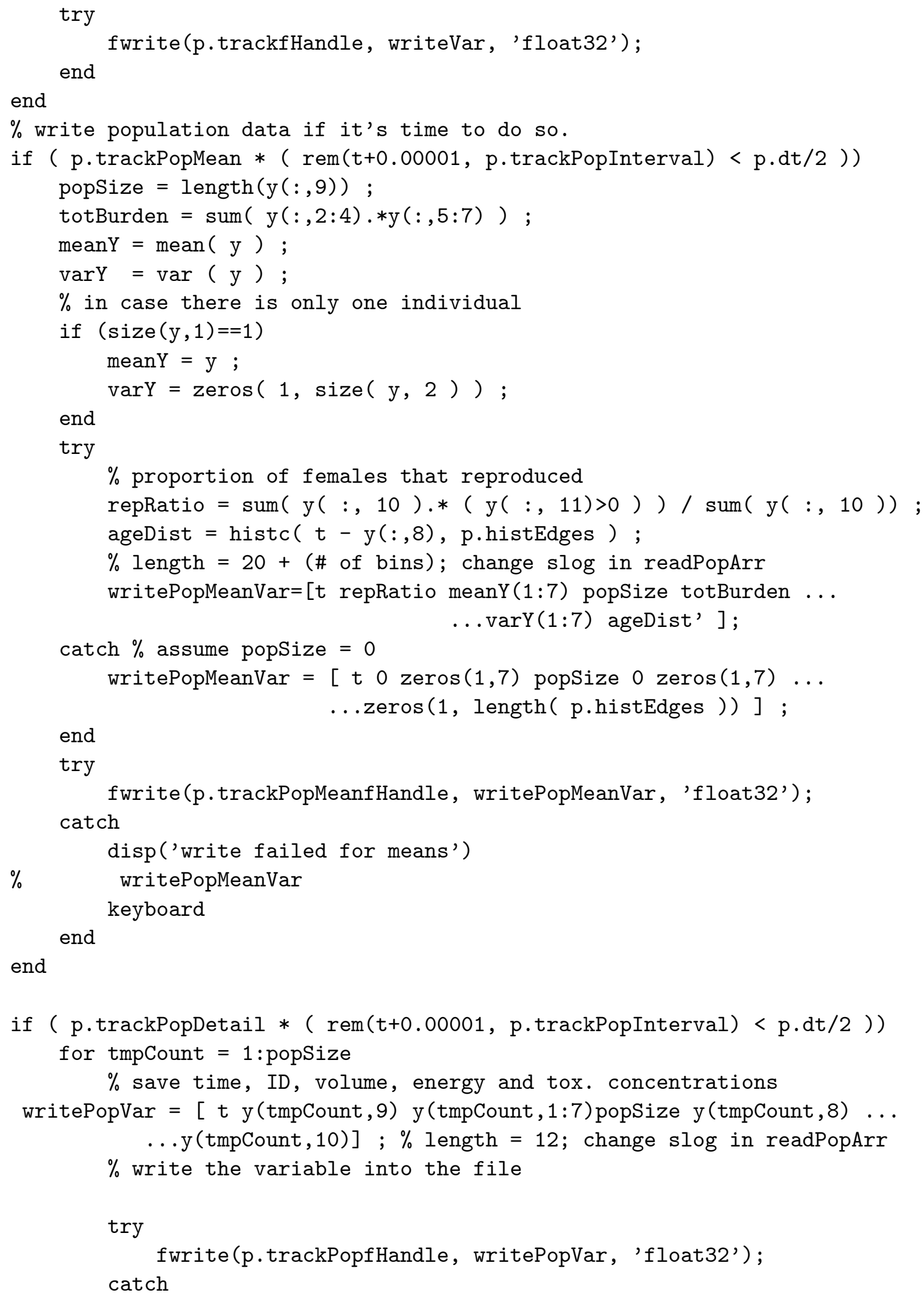




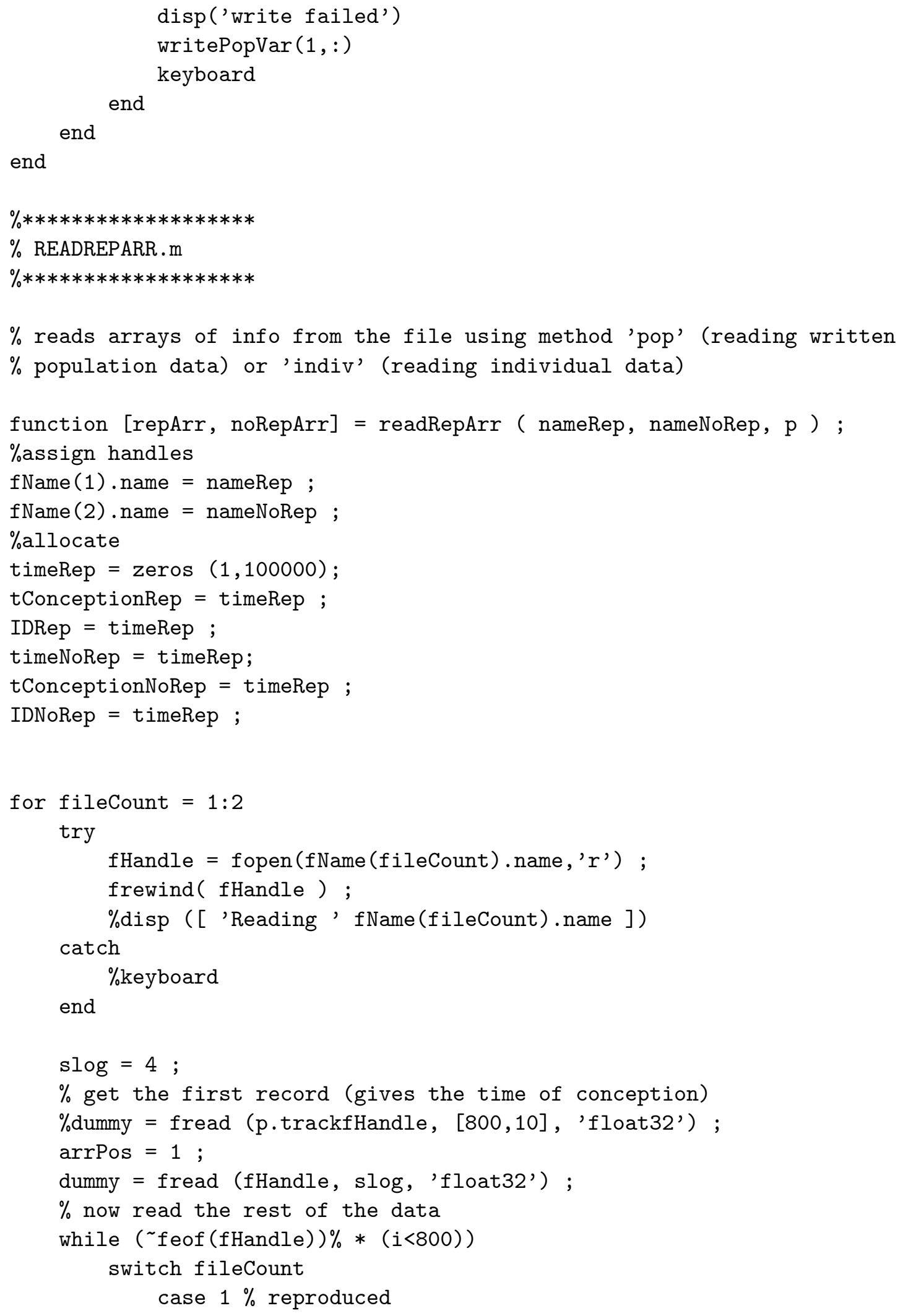




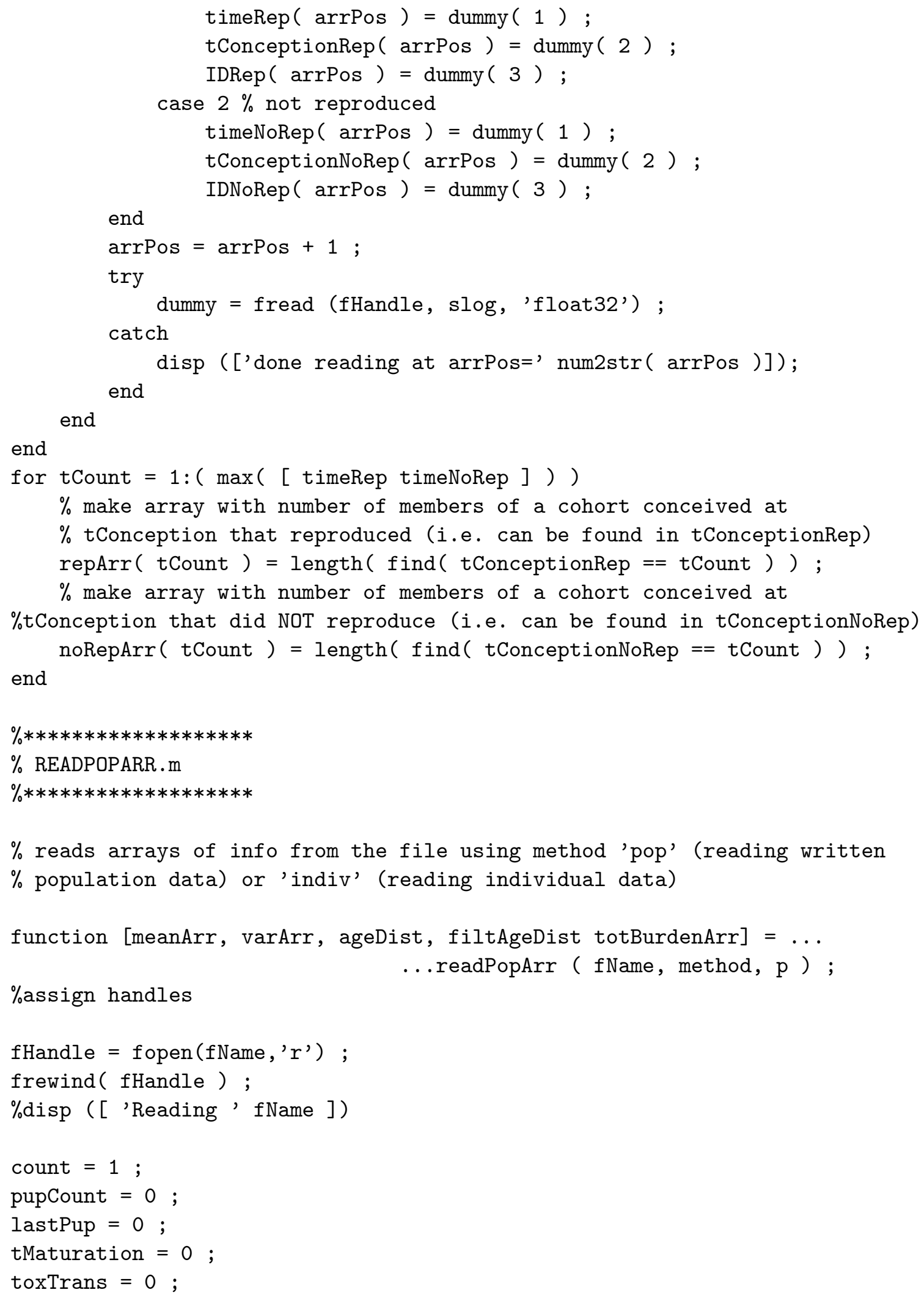




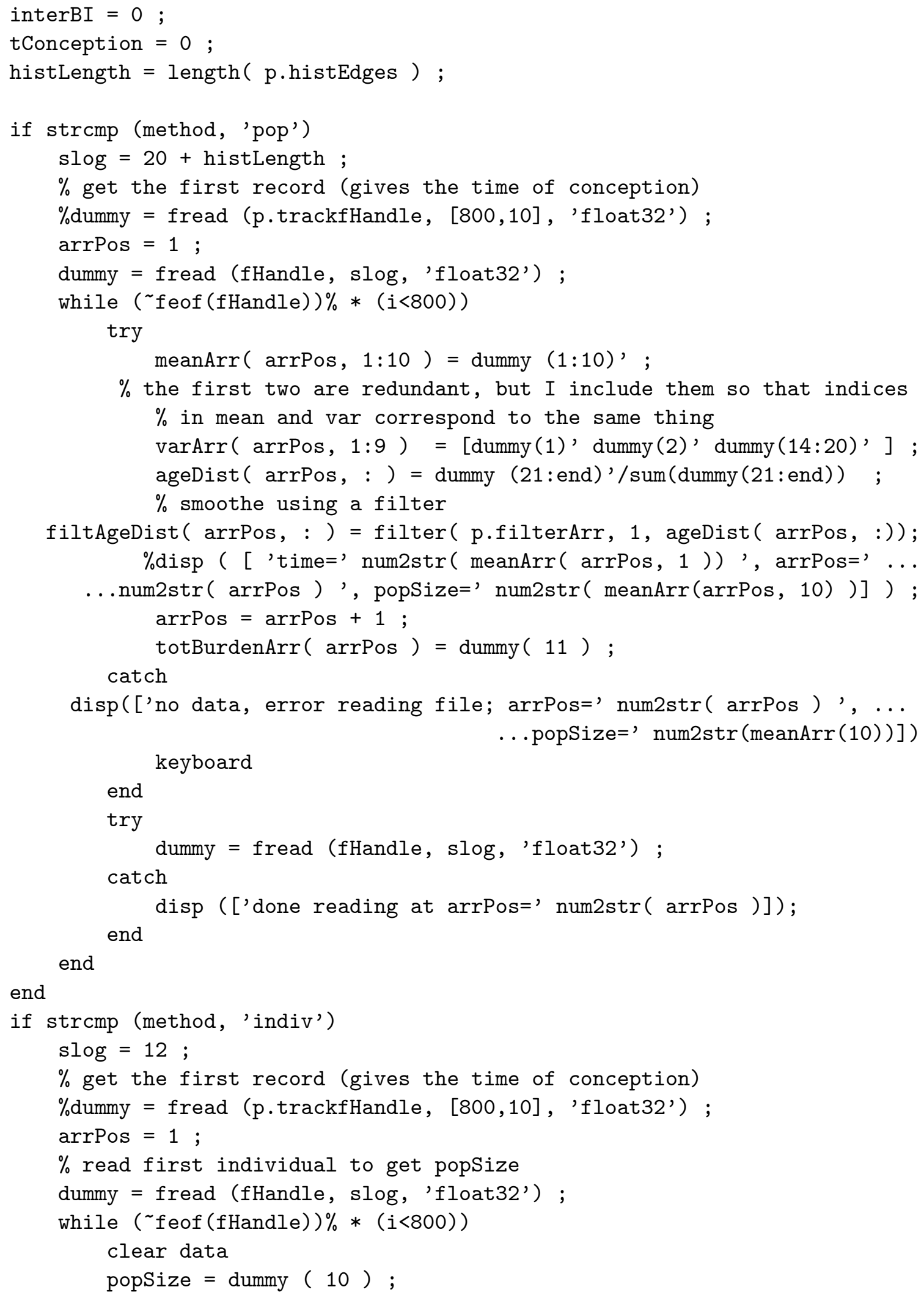




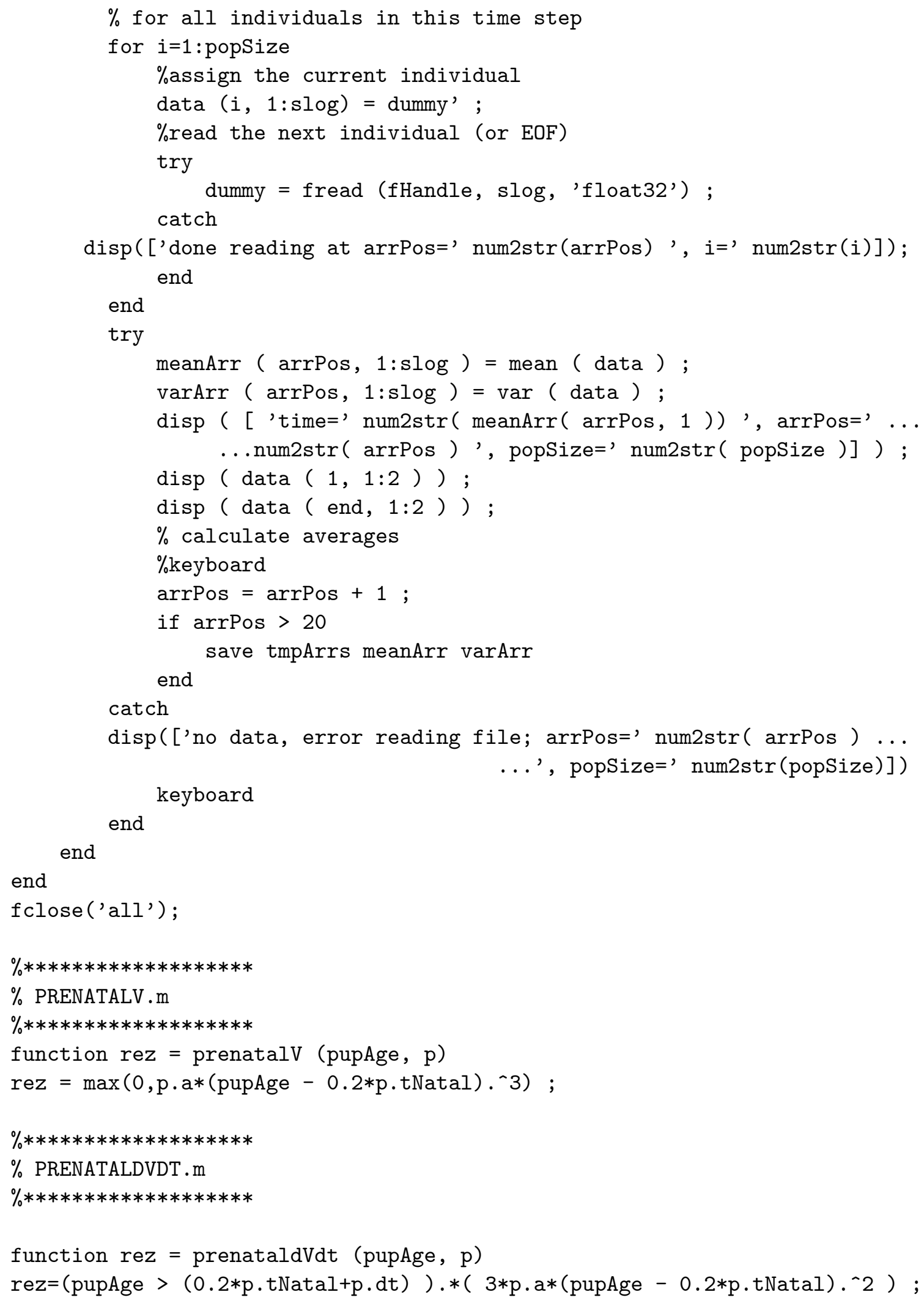




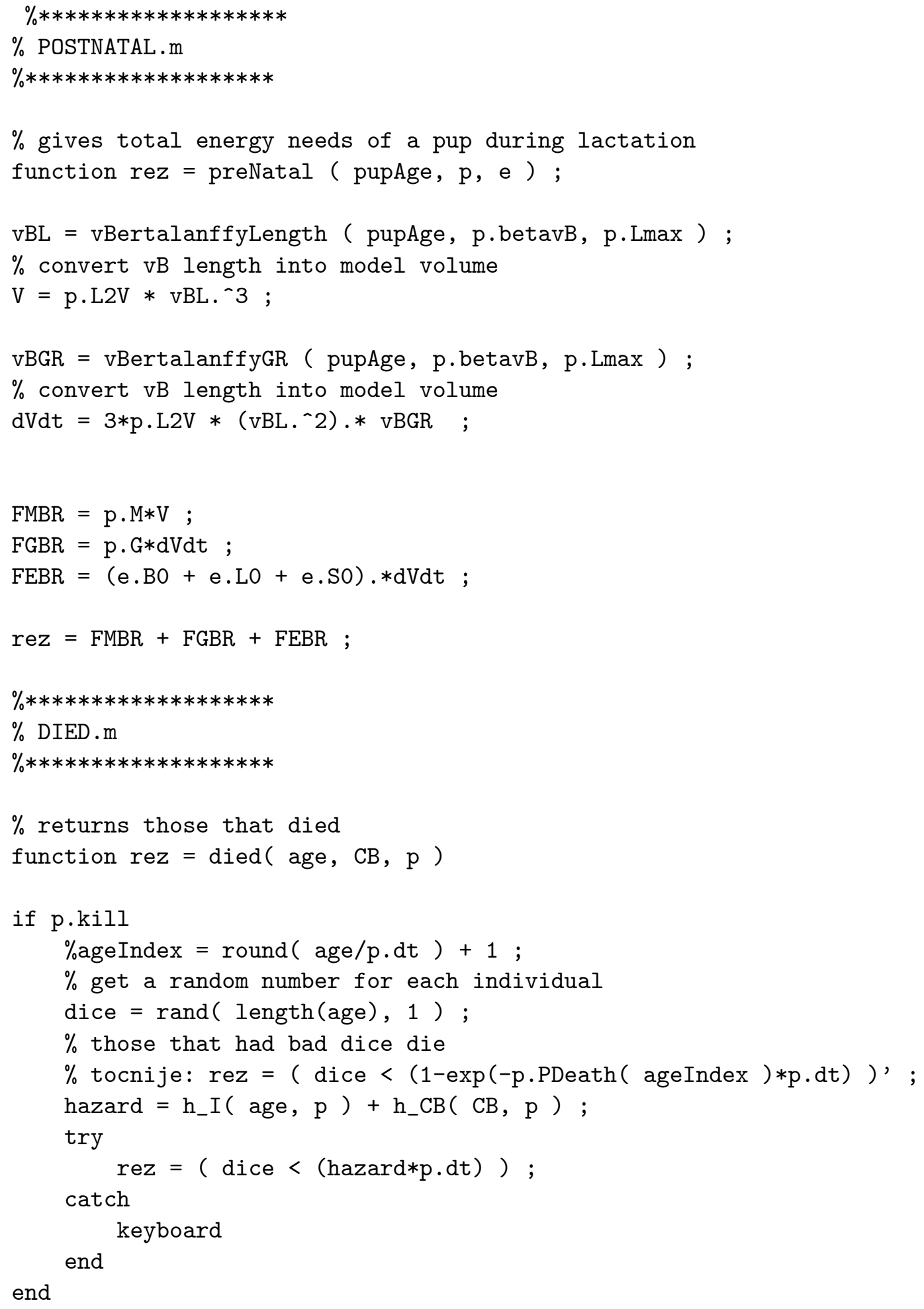




\section{Chapter 4}

\section{Integrating Dynamic Energy Budgets into Matrix Population Models}

The work in this chapter has been accepted for publication by Ecological Modelling as

Klanjscek, T., H. Caswell, M.G. Neubert and R.M. Nisbet.

Integrating Dynamic Energy Budgets into Matrix Population Models

\subsection{Abstract}

Population dynamics depend on the growth and reproduction of individuals, which are dictated by energy intake and budgeting. Here we show how to construct a simple matrix population model from a dynamic energy budget model in a constant or seasonally variable environment. The matrix model accurately predicts asymptotic population dynamics for a wide range of parameter values and environmental conditions. The model captures some transients well, but more elaborate stage structure is necessary when the initial age distribution within stages is far from the stable age distribution. 


\subsection{Introduction}

Food availability and the resulting energy intake and budgeting are major factors affecting growth and reproduction of individuals and, therefore, population dynamics. To investigate these effects, one needs demographic models that incorporate energy budgeting. In this paper, we use a physiological model describing individual energy acquisition, energy allocation, growth and reproduction to calculate the vital rates of a simple demographic model.

When constructing such coupled energy budget/demographic models, one must make two choices. First, one must choose a description of the energy budget of an individual. We chose a simple model based on the most comprehensive mathematical theory currently available: the dynamic energy budget (DEB) theory (Kooijman 2000; see Section 4.2.1). This model has the advantage of being a "supply-side model" in which food availability determines growth, survival and reproduction. As such it enables one to analyze the interacting effects of physiology and environmental variability on population dynamics - effects that we hope to study in the future.

Second, one must pick a framework for describing the demography. Previous theoretical studies have used continuous-time ordinary, delay, or partial differential equations for this purpose. This is a mathematically natural choice because energybudget models typically treat time as continuous variable. Practical or biological circumstances, however, often make discrete-time matrix population models more convenient, hence their frequent use in empirical population ecology (Caswell, 1989; Caswell, 1996; Ripley and Caswell 2005). Despite their popularity, we know of no supply-side energy budget model linked with a matrix population model. Here, we have chosen a simple two-stage matrix model (described in Section 4.2.2) for our demographic model partly to demonstrate how to make such a link (in Section 4.3). We provide a recipe for linking more general energy budget models with more general matrix population models in Appendix A.

In Section 4.4 we compare our model's demographic predictions to simulations 
of an individual based model (IBM) in which individuals are governed by the same physiological model. It turns out that the model accurately captures both long and short-term population dynamics when the population is initially close to the stable age distribution. When the population is not initially close to the stable age distribution, our model and the IBM may disagree; we describe the reasons for this disagreement, and how it can be ameliorated, in the Discussion (Section 4.5).

\subsubsection{The DEB model}

Energy budget models describe the acquisition and utilization of energy by individuals for maintenance, growth and reproduction (e.g. Ren and Ross 2001). We constructed a simple DEB model based on Kooijman (2000) and Muller and Nisbet (2000). The model describes the energy budget of an ectothermic organism that grows, matures at a fixed size, and reproduces in discrete periodic events during the breeding season (birth-pulse reproduction). For a comprehensive review of DEB models and their applications, see Nisbet et al. (2000).

In this DEB model, the organism is partitioned into two compartments: structure and energy reserves. The exact composition of these compartments depends on the species. In general, the reserves are materials that can be utilized as an energy source by the organism (e.g. non-structural lipids, glucose and some proteins). The remaining tissue (e.g. bones, muscle, structural lipids etc.) compose the structure compartment. Fueled by the energy from the reserves, the structure enables the organism to interact with its environment and feed.

Organisms assimilate acquired food into the energy reserves, or excrete it as feces. They utilize energy at a rate determined by the energy stored in the reserves. According to Kooijman's " $\kappa$-rule" (Kooijman, 2000), organisms allocate a fraction $\kappa$ of the utilized energy to growth and somatic maintenance (i.e. increase and maintenance of the structure), and the remaining fraction to maturation or reproduction, and to maintaining the acquired level of maturity (Figure 4-1). Somatic maintenance takes 


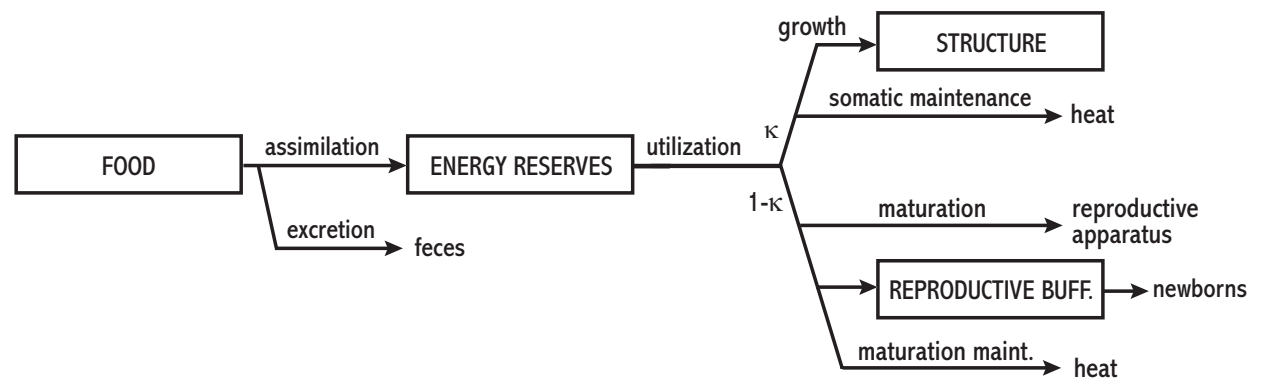

Figure 4-1: The fluxes of energy in a $\kappa$-rule DEB model. Energy from food is assimilated, stored into energy reserves, and then utilized. A proportion $\kappa$ of the utilized energy is spent on growth and somatic maintenance. A proportion $(1-\kappa)$ is either spent on maturation and maturation maintenance, or stored into a reproductive buffer and spent on reproduction. In case of energy shortage, the somatic maintenance has the absolute priority in energy allocation. Boxed compartments are explicitly modeled in the DEB. Structure and newborns, although not measured in units of energy, represent sinks of energy.

precedence over any other energy need. If the energy that can be extracted from the reserves is not sufficient to satisfy the somatic maintenance, the organism dies.

We use $V$ (structural volume) and $e$ (energy density $[E]$ relative to maximum energy density $\left[E_{m}\right]$ ) as state variables that describe the structure and reserves of an individual of age $s$. Throughout the text we simplify the discussion by treating $V$ as a measure of the size of the structure of the organism. See Table 4.2 for a description of all the state variables and parameters of the model, and Table 4.1 for the parameter values we used as a starting point for our analyzes. A sample solution of the DEB model is given in Figure 4-2.

\section{Growth}

The rate of energy intake $f$, which is measured relative to its maximum value, is a function of food availability (Kooijman, 2000). The rate of change of scaled reserve density, $e$, is proportional to the difference between $f$ and $e$, where the constant of 
Table 4.1: Standard parameter values

\begin{tabular}{cc}
\hline Parameter & Value \\
\hline \hline$\kappa$ & 0.8 \\
\hline$\kappa_{R}$ & 0.001 \\
\hline$v$ & $0.075 \mathrm{my}^{-1}$ \\
\hline$m$ & $0.58 \mathrm{y}^{-1}$ \\
\hline$g$ & 0.25 \\
\hline$V_{b}$ & $10^{-9} \mathrm{~m}^{3}$ \\
\hline$V_{p}$ & $1.7310^{-6} \mathrm{~m}^{3}$ \\
\hline$\left[E_{m}\right]$ & $0.7 a J\left(^{+}\right)$ \\
\hline$h_{a}$ & $0.15 \mathrm{~m}^{3} d_{D N A}^{-1} y^{-1}\left(^{++}\right)$ \\
\hline$f$ & 0.5 \\
\hline
\end{tabular}

${ }_{+}$converts a chosen proxy for energy (dry weight in Muller and Nisbet (2000)) into Joules, and cancels out after parameterization (Fujiwara et al., 2004).

${ }^{++} d_{D N A}$ is a unit of the damage-inducing compound which cancels out in calculations.
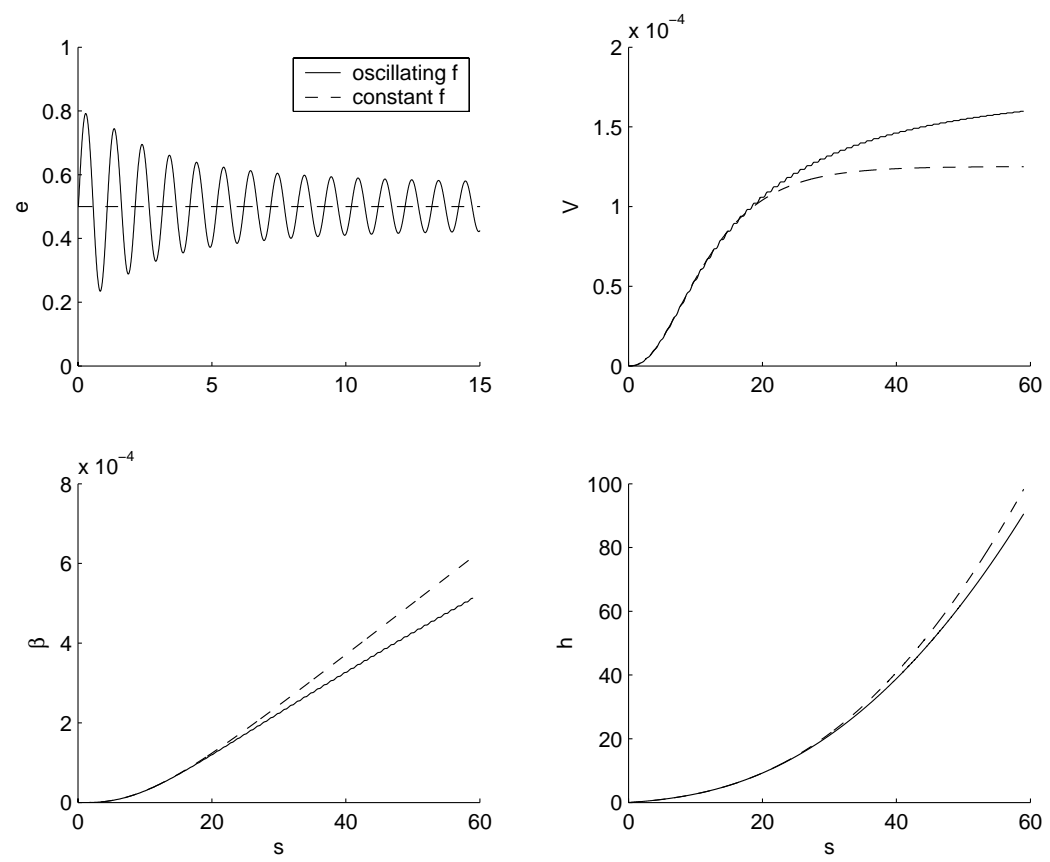

Figure 4-2: Sample solutions of the DEB model as a function of age $(s)$ using the parameter values from Table 4.1 for constant energy intake $(f(s)=0.5)$ and oscillating energy intake $(f(s)=0.5+0.3 \sin (2 \pi s))$. In the fluctuating environment, the organism grows larger, commits less energy to reproduction, and accumulates less damage (lower $h(s))$. For additional discussion see Muller and Nisbet (2000). 
Table 4.2: State variables as a function of age $(s)$ and parameters of the DEB model. For further discussion see Kooijman (2000).

\begin{tabular}{|c|c|c|}
\hline descriptor & dimension & description \\
\hline $\bar{V} \overline{V(s)}$ & $\overline{l e n g t h^{3}}$ & " volume of the structure compartment \\
\hline$\left[E_{m}\right]$ & $\frac{\text { energy }}{\text { length }}$ & maximum energy reserve density \\
\hline$[E]$ & $\frac{\text { energy }}{\text { length }}$ & energy reserve density \\
\hline$e(s)$ & - & scaled energy density in the energy reserves, $\frac{[E]}{\left[E_{m}\right]}$ \\
\hline$\beta(s)$ & energy & cumulative energy committed to reproduction \\
\hline$Q(s)$ & mass & mass of damage-inducing compound \\
\hline$h(s)$ & $\frac{\text { probability }}{\text { time }}$ & hazard rate: probability of death per unit of time \\
\hline$\kappa$ & - & energy partitioning coefficient \\
\hline$\kappa_{R}$ & - & $\begin{array}{l}\text { fraction of reproduction energy } \\
\text { realized in a newborn }\end{array}$ \\
\hline$v$ & $\frac{\text { length }}{\text { time }}$ & energy conductance \\
\hline$m$ & time $e^{-1}$ & $\begin{array}{l}\text { maintenance rate coefficient: cost of maintenance } \\
\text { relative to cost of growth }\end{array}$ \\
\hline$g$ & - & $\begin{array}{l}\text { energy investment ratio: cost of growth relative } \\
\text { to maximum avail energy for growth }\end{array}$ \\
\hline$\eta$ & $\frac{\text { mass }}{\text { energy }}$ & $\begin{array}{l}\text { mass-energy coupler: damage-inducing compound } \\
\text { accumulated per energy respired }\end{array}$ \\
\hline$\overline{V_{b}}$ & length ${ }^{3}$ & structural volume at birth \\
\hline$V_{p}$ & length ${ }^{3}$ & structural volume at maturation \\
\hline$V_{m}$ & length $h^{3}$ & maximum structural volume $\left(\frac{v}{m g}\right)^{3}$ \\
\hline$C_{N}$ & energy & effective energy cost of a newborn female \\
\hline$h_{a}$ & $\frac{\text { probabab. length }}{\text { mass time }}$ & ageing acceleration (the rate of increase of $h$ ) \\
\hline$f$ & - & energy intake scaled to maximum energy intake \\
\hline
\end{tabular}


proportionality $v$ is called the energy conductance:

$$
\frac{d e}{d s}=v(f-e) V^{-\frac{1}{3}} .
$$

The rate of increase of structure in DEB theory is determined by the energy allocated to growth and somatic maintenance, after the somatic maintenance has been met,

$$
\frac{d V}{d s}=\max \left(\frac{m g}{e+g}\left(e V_{m}^{\frac{1}{3}} V^{\frac{2}{3}}-V\right), 0\right) .
$$

Note that the size of the structure cannot decrease.

\section{Reproduction}

We assume that the organism is mature, and allocates energy to reproduction, whenever $V \geq V_{p}$. In mature organisms, the energy flux to reproductive processes (including maturation) is

$$
E_{R}=\left\{\begin{array}{ll}
(1-\kappa)\left[E_{m}\right] \frac{e g}{e+g}\left(v V^{\frac{2}{3}}+m V\right) & \text { when } \frac{d V}{d s}>0 \\
{\left[E_{m}\right]\left(v e V^{\frac{2}{3}}-m g \kappa V\right)} & \text { when } \frac{d V}{d s}=0
\end{array},\right.
$$

and the energy flux required to maintain maturity is

$$
E_{M}=(1-\kappa)\left[E_{m}\right] m g V_{p} .
$$

Hence, the flux of energy to the reproduction buffer $(\beta)$ is

$$
\frac{d \beta}{d s}=\max \left(E_{R}(V)-E_{M}, 0\right) .
$$

We assume that the energy buffer is emptied and utilized for reproduction at each breeding event, if the mother accumulated enough energy to produce at least one newborn. 


\section{Mortality}

Probability that the organism dies by age $s+d s$, given that it survived until age $s$, is proportional to the hazard rate $h(s)$ :

$$
P(\text { dead at } s+d s \mid \text { alive at } s)=h(s) d s .
$$

The hazard rate increases at a rate proportional to the density of damage-inducing compounds within the organism $(Q / V)$ :

$$
\frac{d h(s)}{d s}=h_{a} \frac{Q}{V}
$$

Damage inducing compounds are generated by respiration:

$$
\frac{d Q}{d s}=\eta g\left[E_{m}\right]\left(\frac{d V}{d s}+m V\right) .
$$

Since $h(s)$ is proportional to both $\eta$ and $h_{a}$, we can simplify analysis if assume that $\eta$ is unity and vary only $h_{a}$ in the analyses. The hazard rate and the mass of damageinducing compounds are assumed negligible in a newborn individual, so we set these variables to zero at birth.

Throughout the paper we use the parameter values mostly obtained from a DEB model for the mussel Mytilus edulis (Table 4.1) (Muller and Nisbet, 2000). The value for $\left[E_{m}\right]$ was not available in Muller and Nisbet (2000); we choose the one that approximately matches the median value used by Fujiwara et al. (2004).

\subsubsection{The matrix population model}

Matrix population models are discrete-time structured models that divide population into discrete stages, and describe evolution of the population through these stages through time:

$$
\mathbf{n}(t+\alpha)=\mathbf{A n}(t)
$$


A is a projection matrix, $\alpha$ is the projection interval, and the vector $\mathbf{n}(t)$ gives the distribution of individuals among stages at time $t$.

Although most applications require more complicated structures, as a test case we used a two-stage matrix model that distinguishes between immature and mature individuals. Individuals become mature when they reach a minimal reproductive size $\left(V_{p}\right)$.

The projection matrix is

$$
\mathbf{A}=\left[\begin{array}{cc}
P_{11} & F \\
P_{21} & P_{22}
\end{array}\right]
$$

We assumed a birth-pulse population, with births occurring at the end of the projection interval. The transition probabilities depend on $\gamma$, the maturation probability, and on $\sigma_{1}$ and $\sigma_{2}$, the survival probabilities in stages 1 and 2:

$$
\begin{aligned}
& P_{11}=\sigma_{1}(1-\gamma), \\
& P_{21}=\sigma_{1} \gamma, \\
& P_{22}=\sigma_{2} .
\end{aligned}
$$

We assume that the maturation probability is independent of survival, and that the projection interval is less than the age at maturity.

If all vital rates are constant, the asymptotic growth rate of the population is given by the dominant eigenvalue $\lambda_{1}$ of the projection matrix $\mathbf{A}$, and the intrinsic rate of increase is

$$
r=\frac{1}{\alpha} \ln \lambda_{1}
$$

The stable stage distribution is given by the right eigenvector $\mathbf{w}_{1}$ associated with $\lambda_{1}$. Other demographic statistics that can be obtained from the model include the reproductive value $\left(\mathbf{v}_{1}\right.$, the left eigenvector associated with $\left.\lambda_{1}\right)$, and sensitivities of the growth rate to changes of the elements in the matrix. 


\subsection{Methods}

In this section we derive the matrix model from solutions of the DEB model. We assume that food availability $f(t)$ is either constant or periodic with a period equal to the projection interval (this might represent seasonal variability, for example).

Under these assumptions, for a given $f(t)$, the age $(s)$ of an individual completely determines its size, the contents of its reproductive buffer, and how likely it is to die.

Individuals of different ages within each stage of the matrix model have different probabilities of survival and rates of growth and reproduction. Hence, the parameters for each stage are a weighted average of the DEB solutions. We use the stable age distribution within a stage as the weighting function, although this will be strictly correct only when the distribution is stable.

The stable age distribution is

$$
\phi(s)=K \lambda_{1}^{-\frac{s}{\alpha}} \exp \left(-\int_{0}^{s} h(\xi) d \xi\right),
$$

where $K$ is the ratio of number of newborns to the total population size in the stable age distribution (see Appendix B).

\subsubsection{Maturation probability $(\gamma)$}

Growth in the DEB model is deterministic. Since the period of food availability is equal to one projection interval, all individuals experience the same food at the same age and, consequently, reach the reproductive size $\left(V_{p}\right)$ and become mature at the same age, $\tau_{p}$. Therefore, organisms in stage $i$ are of age $s \in\left[\tau_{i}, \tau_{i+1}\right)$. In our matrix model, $\tau_{1}=0, \tau_{2}=\tau_{p}$ and $\tau_{3}=\infty$.

We have assumed that organisms cannot grow from birth to maturity in less than one projection interval, so we restrict our attention to

$$
\tau_{p} \geq \alpha
$$


Therefore, given that they survive, all individuals in stage $i$ with ages

$$
\left(\tau_{i+1}-\alpha\right) \leq s<\tau_{i+1}
$$

will grow into stage $i+1$ in one projection interval. The probability of growing from stage $i$ into the next stage, conditioned on survival, is therefore

$$
\gamma_{i+1, i}=\frac{\int_{\tau_{i+1}-\alpha}^{\tau_{i+1}} \phi(s) d s}{\int_{\tau_{i}}^{\tau_{i+1}} \phi(s) d s} .
$$

In our matrix model, individuals can only mature from the immature to the mature stage. Hence, the maturation probability

$$
\gamma=\frac{\int_{\tau_{p}-\alpha}^{\tau_{p}} \phi(s) d s}{\int_{0}^{\tau_{p}} \phi(s) d s} .
$$

\subsubsection{Survival probabilities $(\sigma)$}

Individuals in stage $i$ at time $t$ have age $s \in\left[\tau_{i}, \tau_{i+1}\right)$. We assume their age distribution is $\phi(s)$. After one projection interval, all individuals grow older by $\alpha$ and have the distribution $\phi(s) e^{r \alpha}$. Survivorship is given by the ratio of number of individuals who lived through the projection interval and the initial number of individuals,

$$
\begin{aligned}
& \sigma_{i}=\frac{\int_{\tau_{i}+\alpha}^{\tau_{i+1}+\alpha} \phi(s) e^{r \alpha} d s}{\int_{\tau_{i}}^{\tau_{i+1}} \phi(s) d s} \\
&=\lambda_{1} \frac{\int_{\tau_{i}+\alpha}^{\tau_{i+1}+\alpha} \phi(s) d s}{\int_{\tau_{i}}^{\tau_{i+1}} \phi(s) d s} .
\end{aligned}
$$




\subsubsection{Fecundity $(F)$}

We estimate the flux of energy to the reproductive buffer as the average flux within the stage.

$$
\overline{\Delta \beta_{i}}=\alpha \frac{\left.\int_{\tau_{i}}^{\tau_{i+1}} \phi(\xi) \frac{d \beta(s)}{d s}\right|_{s=\xi} d \xi}{\int_{\tau_{i}}^{\tau_{i+1}} \phi(s) d s} .
$$

If $C_{N}$ is the energy cost of a newborn, one adult produces

$$
N_{2}=\frac{\overline{\Delta \beta}_{2}}{C_{N}}
$$

newborn females on average during each breading season. Considering both energetics and demography, the energetic cost of one newborn female is

$$
C_{N}=\frac{1}{\kappa_{R}}\left[E_{m}\right](\kappa g+e) V_{b}
$$

This cost includes the energy used to create its structure (proportional to $\left[E_{m}\right] V_{b}$ ), the energy given to it by its mother $\left(e\left[E_{m}\right] V_{b}\right)$. Furthermore, there is a cost associated with the tissue mother discards at birth, and the demographic reality that only a fraction of newborns are female and survive. This cost is represented by $\kappa_{R} \in(0,1]$ in equation (4.24).

Our model ignores respiration of the structure of the newborn prior to birth, possibly variable duration of pre-natal development and other less significant factors. The form we use is simple, but still elaborate enough to capture the increase in energy transfer from mother to child as the energy density of the mother $(e)$ increases. We assumed that the young were born with energy density equal to the mother's averaged over the projection interval.

Fecundity $(F)$ is the number of newborns at time $t+\alpha$ per adult at time $t$. In our birth-pulse population, it is the rate of production of newborns per adult that 


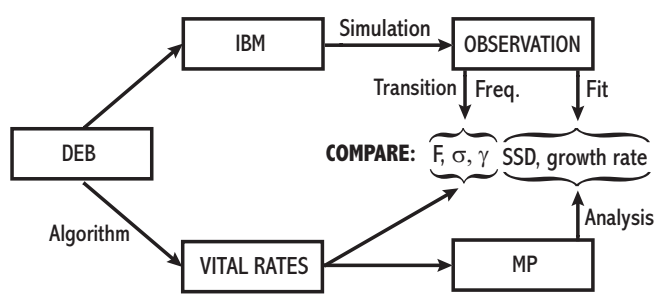

Figure 4-3: Schematic of the assessment process. We created an IBM based on the DEB model. We fitted the growth rate and stable stage distribution (SSD) to the observed population dynamics, and used the transition frequencies observed in a simulation of the IBM to calculate the observed vital rates. At the same time, we arrived at the vital rates through the methods described in sections (4.3.1)-(4.3.3) and calculated the growth rate and SSD from the resulting MP model. The performance of the matrix model has been assessed through comparison between the two sets of vital rates, growth rates and SSDs.

survived to the end of the projection interval $\alpha$ (Caswell, 2001, pp. 172-173),

$$
F=\sigma_{2} N_{2}
$$

Equations (4.19), (4.21) and (4.25) provide the necessary vital rates to write down the projection matrix (4.10). However calculating $\phi(s)$ requires a value for $\lambda_{1}$. We can arrive at $\lambda_{1}$ through an iterative process: start with $\lambda_{1}=1$, find the matrix model and calculate the growth rate $\lambda_{1}^{\prime}$. Repeat the process with $\lambda_{1}=\lambda_{1}^{\prime}$ until the desired accuracy is reached (Caswell, 2001, pp. 164). In this paper we required that the growth rate converge to the accuracy of $10^{-3}$.

\subsubsection{The individual-based model (IBM)}

To assess the ability of the matrix model to capture the dynamics of a population, we compared its asymptotic and transient dynamics with the simulations of an individual based model (IBM) in which individuals grow according to the DEB (Figure 4-3).

In the IBM, at each time step individuals of age $s$ grow older by $d s$, grow by $d V$, die with probability $h(s) d s$, and commit $d \beta(s)$ energy to reproduction. Every 
projection interval ( $\alpha$ units of time) individuals reproduce according to the energy stored in the reproductive buffer.

We calculated the number of newborn females produced by one individual female in one breeding season $(N)$ from the ratio of the energy accumulated since the last reproduction $(\Delta \beta)$ and the energy needed to produce one newborn female $\left(C_{N}\right)$. The ratio is not an integer, whereas $N$ needs to be. Therefore, we have to round the ratio so that the expected number of newborns is equal to it. Denoting the integer part of the ratio as floor $\left(\frac{\Delta \beta}{C_{N}}\right)$, the number of female newborns for each individual is

$$
N=\left\{\begin{array}{ll}
\text { floor }\left(\frac{\Delta \beta}{C_{N}}\right)+1 & \text { with probability }\left(\frac{\Delta \beta}{C_{N}}-\text { floor }\left(\frac{\Delta \beta}{C_{N}}\right)\right) \\
\text { floor }\left(\frac{\Delta \beta}{C_{N}}\right) & \text { with probability }\left(1-\left(\frac{\Delta \beta}{C_{N}}-\text { floor }\left(\frac{\Delta \beta}{C_{N}}\right)\right)\right)
\end{array} .\right.
$$

We eased the computational requirements by treating individuals with age differences less than $d s=0.01$ as having the same age. Because reproduction is seasonal, and all individuals reproduce at the same time, the resulting age distribution is 'spiked', i.e. individuals can have only discrete ages corresponding to the reproductive events. This is in contrast with the continuous age distribution that has been assumed in the matrix model.

From the IBM simulations, we measured population growth rate and the stable stage distribution (SSD) and compared them to those predicted by the matrix model. We also measured $\sigma_{1}, \sigma_{2}$, and $\gamma$ from the the IBM. Let the number of transitions from stage $j$ to stage $i$ be $n_{i j}$, where stages 1,2 , and 3 are immature, mature and dead individuals, respectively (Table 4.3). Then

$$
\begin{aligned}
\hat{\sigma}_{1} & =1-\frac{n_{31}}{n_{11}+n_{21}+n_{31}} \\
\hat{\sigma}_{2} & =1-\frac{n_{32}}{n_{22}+n_{12}+n_{32}} \\
\hat{\gamma} & =\frac{n_{21}}{\left(n_{11}+n_{21}\right)} \\
\hat{F} & =\frac{n_{12}}{n_{22}+n_{32}} .
\end{aligned}
$$


Table 4.3: Transition counts $n_{i j}$ from stage $j$ to stage $i$.

\begin{tabular}{lll}
\cline { 2 - 3 } immature $(i=1)$ & $\begin{array}{l}\text { immature }(j=1) \\
\text { did not mature }\left(n_{11}\right)\end{array}$ & mature $(j=2)$ \\
\hline mature $(i=2)$ & immature that matured $\left(n_{21}\right)$ & mature that survived $\left(n_{22}\right)$ \\
\hline dead $(i=3)$ & immature that died $\left(n_{31}\right)$ & mature that died $\left(n_{32}\right)$ \\
\hline
\end{tabular}

The asymptotic comparisons of the rates and demographic statistics required that the population converge to a stable age distribution. We ran the simulations for 130 units of time or until the population went extinct. We discarded the first $10 \%$ and the last $20 \%$ of the simulated time interval to allow for as much convergence as possible before the onset of low-number effects, and used the remaining data as observations for comparison. We expedited convergence by using the equation (4.15) to distribute the initial 1000 individuals through ages using the predicted $\lambda_{1}$. The Convergence, however, was not always attained, especially for parameter values that produced very small growth rates. If the initial 1000 individuals did not a provide sufficient number of observations to measure the vital rates, we used an initial population of $10^{6}$ individuals.

\subsection{Results}

\subsubsection{Long term (asymptotic) dynamics}

In Table 4.4 we compare observations of the IBM and the predictions of the matrix model for the standard parameter values listed in Table 4.1 for $\tau_{p}=3 \alpha$.

Predicted and observed $\gamma$ and $\sigma_{1}$ agree extremely well; even the greatest proportional differences ${ }^{1}$, for $F$ and $\sigma_{2}$, are less than $12 \%$. As a result, the growth rates hardly differ. To see whether these demographic statistics obtained from the matrix model and the IBM agree for a wide range of parameters, we compared the observed

\footnotetext{
${ }^{1}$ proportional difference $=\frac{(\text { observed value })-(\text { predicted value })}{(\text { observed value })}$
} 
Table 4.4: Comparison of predicted and observed demographic statistics and vital rates.

\begin{tabular}{ccc}
\cline { 2 - 3 } & Observed & Predicted \\
\hline \hline$\lambda$ & 1.13 & 1.13 \\
\hline$S S D$ & 0.61 & 0.59 \\
\hline$\gamma$ & 0.26 & 0.26 \\
\hline$\sigma_{1}$ & 0.93 & 0.91 \\
\hline$\sigma_{2}$ & 0.84 & 0.72 \\
\hline$F$ & 0.71 & 0.77 \\
\hline
\end{tabular}

and predicted demographic statistics for different values of the parameters $h_{a}, f, C_{N}$, $\kappa, E_{m}$ and $\alpha$. Unless noted otherwise, while changing one parameter, we used values from Table 4.1 for others.

The results are shown in Figures (4-4)-(4-9). Plot A of each figure compares the growth rates. Plots $\mathrm{B}$ and $\mathrm{C}$ show proportional differences between predicted and observed growth rates, stable stage distributions, fecundities, and transition probabilities. Plot D shows the proportional differences between the observed and predicted survival probabilities.

Population growth rates match very well across all the parameter ranges investigated. The estimates of the vital rates show greatest discrepancies for low growth rates, mainly because populations went extinct in the IBM before reliable observation could be made.

Fecundity was, in general, overestimated. This is notable in Figure 4-4 for large hazard rates: $F$ is overestimated by as much as $7 \%$ for $h_{a}=0.1$ and more than $100 \%$ for large $h_{a}$. The main reason for the overestimate is that when $h_{a}$ is high, individuals in the IBM die before they accumulate enough energy to reproduce, and whatever they have accumulated is lost to the population. The matrix model, however, pools the energy committed to reproduction from all adults, so the accumulated energy for reproduction is not lost. We call this the pooling effect.

The pooling effect is ubiquitous, but more notable when adult life span is short and when it takes longer to accumulate enough energy to reproduce, i.e. when $h_{a}$, 

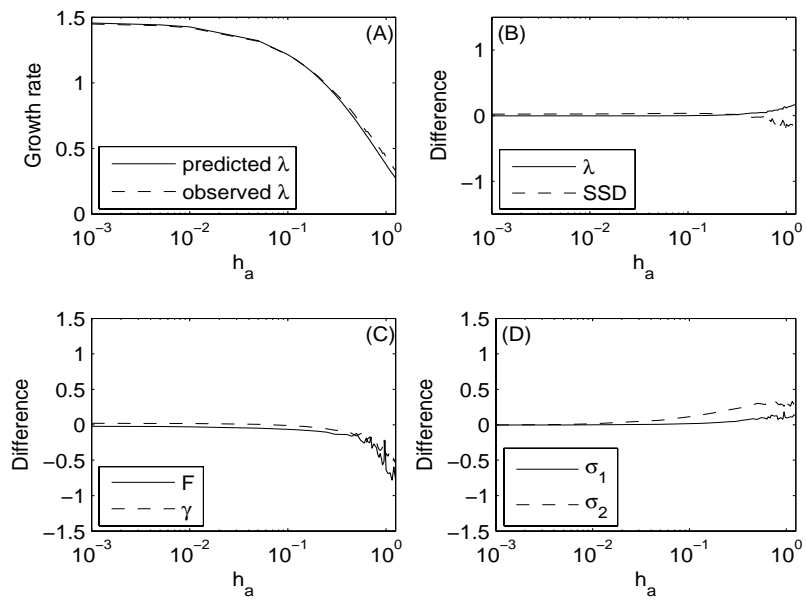

Figure 4-4: Predicted and observed vital rates and demographic statistics versus ageing acceleration $\left(h_{a}\right)$ : the comparison of growth rates $(\mathrm{A})$, proportional difference between the predicted and observed $\lambda$ and $S S D(\mathrm{~B}), F$ and $\gamma(\mathrm{C})$, and $\sigma_{1}$ and $\sigma_{2}$ (D). For $h_{a}<10^{-3}$ the agreement continued to be excellent (tested to $h_{a}=10^{-5}$ ). 

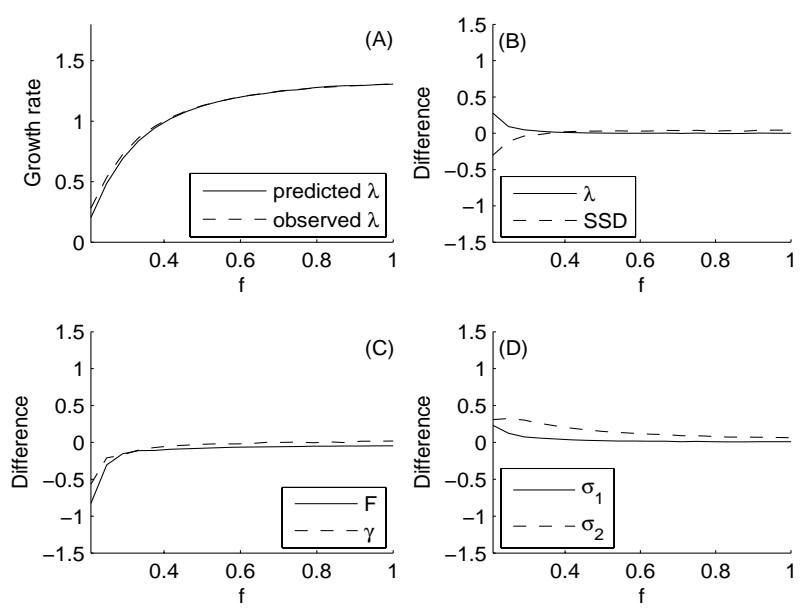

Figure 4-5: Predicted and observed vital rates and demographic statistics versus energy intake $(f)$ : the comparison of growth rates $(\mathrm{A})$, proportional difference between the predicted and observed $\lambda$ and $S S D(\mathrm{~B}), F$ and $\gamma(\mathrm{C})$, and $\sigma_{1}$ and $\sigma_{2}(\mathrm{D})$. For $f<0.2$ populations went extinct too fast to estimate vital rates from observations. 

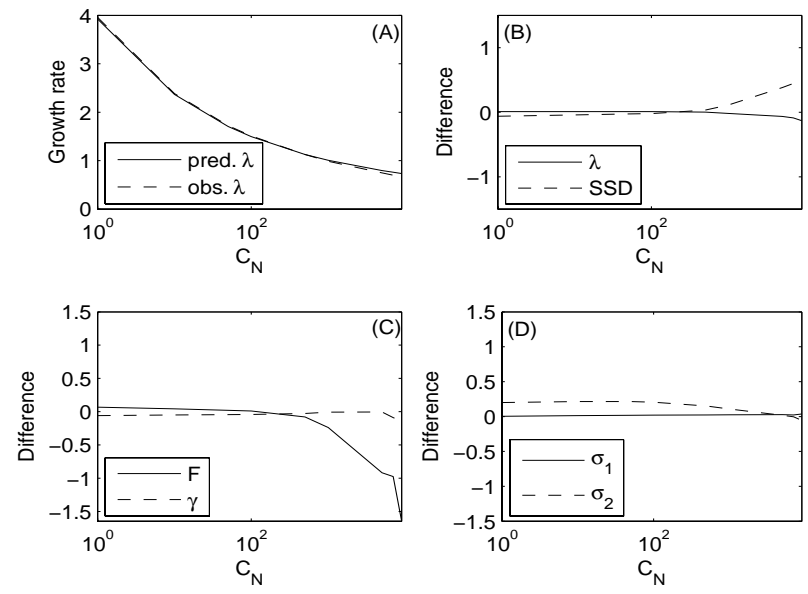

Figure 4-6: Predicted and observed vital rates and demographic statistics versus cost of a newborn $\left(C_{N}\right)$ : the comparison of growth rates $(\mathrm{A})$, proportional difference between the predicted and observed $\lambda$ and $S S D(\mathrm{~B}), F$ and $\gamma(\mathrm{C})$, and $\sigma_{1}$ and $\sigma_{2}$ (D). 

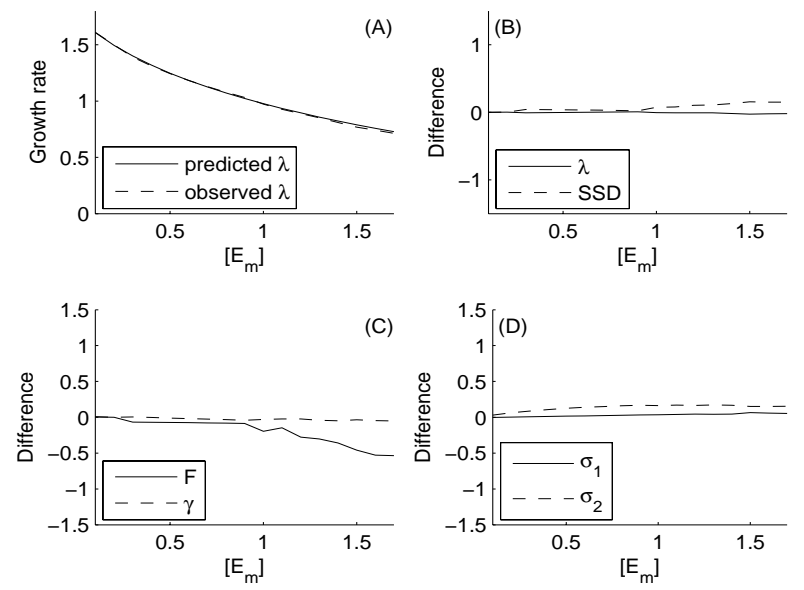

Figure 4-7: Predicted and observed vital rates and demographic statistics versus maximum energy density $\left(\left[E_{m}\right]\right)$ : the comparison of growth rates $(\mathrm{A})$, proportional difference between the predicted and observed $\lambda$ and $S S D(\mathrm{~B}), F$ and $\gamma(\mathrm{C})$, and $\sigma_{1}$ and $\sigma_{2}(\mathrm{D})$. The range was adopted from Fujiwara et al (2004). 

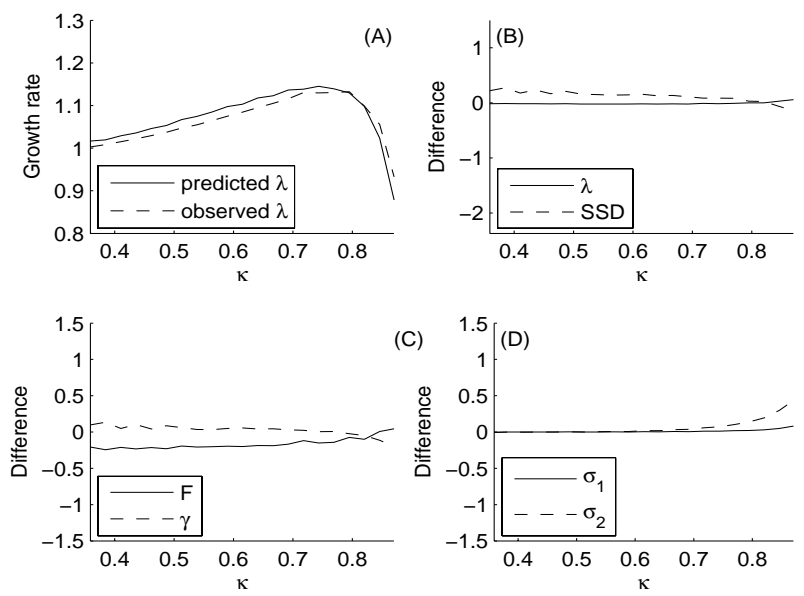

Figure 4-8: Predicted and observed vital rates and demographic statistics versus energy allocation ratio $(\kappa)$ : the comparison of growth rates $(\mathrm{A})$, proportional difference between the predicted and observed $\lambda$ and $S S D(\mathrm{~B}), F$ and $\gamma(\mathrm{C})$, and $\sigma_{1}$ and $\sigma_{2}$ (D). 

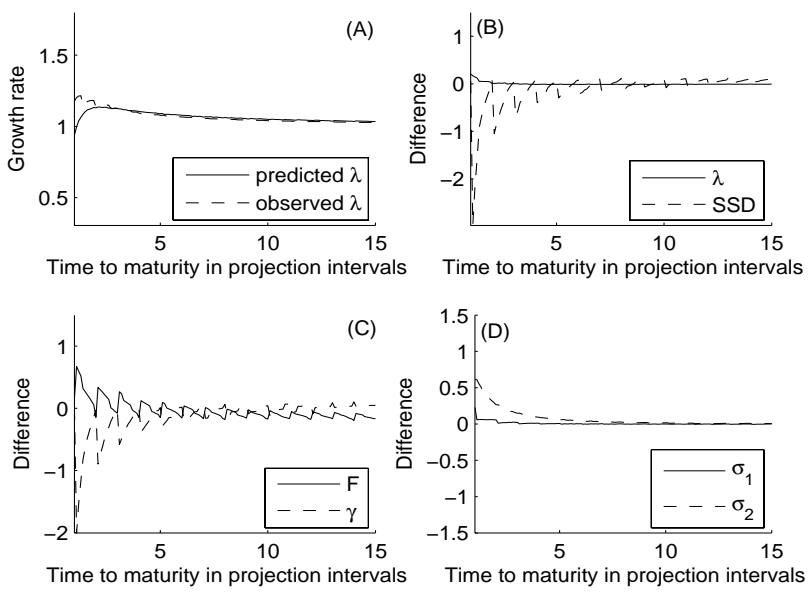

Figure 4-9: Predicted and observed vital rates and demographic statistics versus age to maturity measured in projection intervals $(\alpha)$ : the comparison of growth rates $(\mathrm{A})$, proportional difference between the predicted and observed $\lambda$ and $S S D(\mathrm{~B}), F$ and $\gamma(\mathrm{C})$, and $\sigma_{1}$ and $\sigma_{2}(\mathrm{D})$. 
$C_{N}$ and $\left[E_{m}\right]$ are large, and $f$ and $\kappa$ are small. Since all of these factors decrease the population growth rate, the pooling effect is also more notable for small growth rates.

Figures 4-5 and 4-7 provide further examples of the pooling effect. In Figure 4-5, we overestimate fertility by about $80 \%$ for small $f$ (and small growth rates). In Figure 4-7, the same happens for large $\left[E_{m}\right]$.

In Figure 4-6 we changed the cost of newborns $C_{N}$ by changing the reproductive efficiency $\kappa_{R}$ (4.24). For very large $C_{N}$, both the observed and the predicted growth rates asymptote to the growth rate dictated by mortality alone $(\lambda \approx 0.7)$. Although it should remain constant, the observed survivorship of adults $\left(\hat{\sigma}_{2}\right)$ changes with $C_{N}$. It is not clear why $\hat{\sigma}_{2}$ changes, but huge growth rate and finite possible age of adults are probable causes. When the number of individuals is large and increases quickly, significant rounding due to limited number of digits represented in the computer takes place, influencing the transition counts $\left(n_{i j}\right.$ in 4.27-4.30) and the observed vital rates. Furthermore, when the population is huge, a large number of individuals live to very old age. Consequently, the maximum possible age assumed in the IBM may not be adequate: some individuals may be assumed dead just because they exceed the maximum age assumed by the IBM, thereby increasing adult mortality (decreasing $\left.\hat{\sigma}_{2}\right)$.

The fraction of energy used for growth and somatic maintenance $(\kappa)$ is probably the most important parameter in the DEB theory. In Figure 4-8 we changed $\kappa$ to represent a range of organisms, from reproduction-oriented (small $\kappa$ ) to growth-oriented (large $\kappa$ ). We observed the greatest discrepancies in the stable stage distribution (up to $25 \%$ for small $\kappa$ ), mostly due to similarly large overestimate of fecundity due to the pooling effect.

We varied the relationship between the projection interval and the age at maturity by changing the length of the projection interval (Figure 4-9). A shorter projection interval implies a longer time to maturity measured in projection intervals because the growth of organisms measured in units of time (from the DEB model) is not affected 
by the chosen projection interval $(\alpha)$. The observed ratio of mature to immature individuals shows a sawtooth pattern as the time to maturity deviates from integer multiple of the projection interval.

This is a consequence of spiked age distributions characteristic of birth-pulse populations (discussed in Section 4.3.4). Changing the projection interval changes the ages at which spikes occur at the census time, while the age at maturity always stays the same. The interaction between the two can influence transition counts. For example, if time to maturity is 2.00 projection intervals, a cohort of newborns is counted as mature two censuses later. If the time to maturity is 2.01 projection intervals, the cohort is counted as immature. Neither cohort produces any newborns. Therefore, one can expect large discrepancies when comparing the ratios of individuals in each stage as the time to maturity changes, but not when comparing population-level statistics, such as the growth rate.

\subsubsection{Seasonal environmental variability}

In many systems, the environment varies strongly within any one year, but there is little year to year variation for any given season. Most long-lived organisms have ways of coping with that variability - e.g. energy reserves to carry them through times of scarcity. In this section, we ask if the matrix model can successfully account for such variability. We do this by varying the energy intake $f$ in our DEB model and then assessing its effects on the population growth rate.

If we assume that the projection interval represents one year we can represent seasonal variability by a periodic energy intake with period equal to one year.

$$
f(t)=f_{0}+f_{A} \sin \left(\frac{2 \pi}{\alpha} t\right),
$$

with $f_{A}$ representing the amplitude of oscillations, and average energy intake $f_{0}=0.5$.

We calculated population growth rates (Figure 4-10) for the range of $f_{A}$ from the minimum (constant energy intake, $\left.f_{A}=0\right)$ to the maximum $\left(f_{A}=f_{0}\right)$. We 

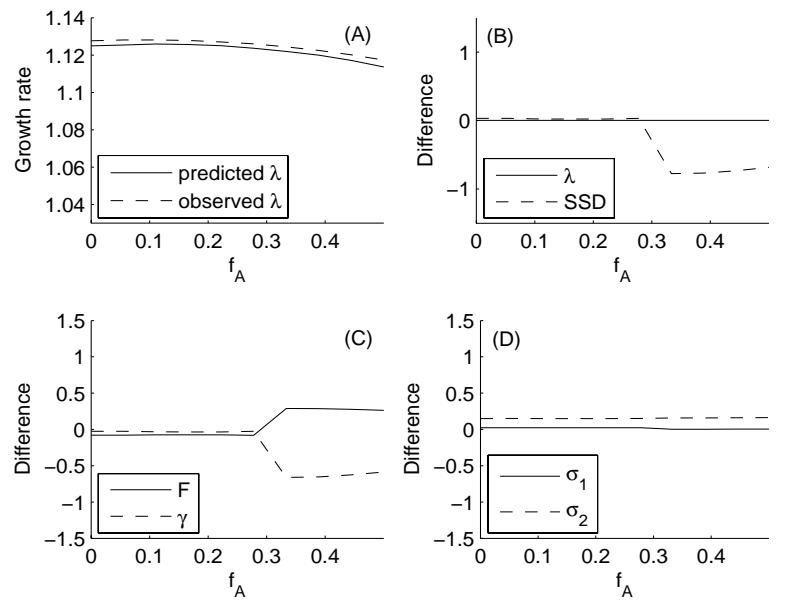

Figure 4-10: Predicted and observed vital rates and demographic statistics versus amplitude of sinusoidal food intake fluctuations $\left(f_{A}\right)$ : the comparison of growth rates (A), proportional difference between the predicted and observed $\lambda$ and $S S D(\mathrm{~B}), F$ and $\gamma(\mathrm{C})$, and $\sigma_{1}$ and $\sigma_{2}(\mathrm{D})$.

assumed that the newborns are born with the average energy density $(e(0)=0.5)$ in the calculations; this assumption guarantees that all individuals follow the same developmental path.

The abrupt changes of the proportional differences in $S S D, F$ and $\gamma$ in Figure (4-10) at $f_{A} \simeq 0.3$ are due to a sudden increase in the observed fecundity and decrease in the observed transition probability as the seasonal variability increases above $f_{A}>$ 0.3. Even though the predicted vital rates do not follow that trend, the predicted and observed population growth rates agree well over the whole range of $f_{A}$.

The growth rate is highest when the seasonal variability is limited, and lowest when the seasonal variability is extreme. In a variable environment, both the energy committed to reproduction and the hazard rate are smaller than in a constant environment (Figure 4-2). For small seasonal variability the benefits of a smaller hazard 
rate outweigh the detriments of a smaller commitment of energy to reproduction; the reverse holds for large seasonal variability.

\subsubsection{Short term (transient) dynamics}

To asses accuracy of the matrix model for predicting transient dynamics we examined two sets of scenarios, loosely corresponding to colonizations and catastrophes. In the colonization scenarios, we initialized the IBM with 1000 newborns or with 1000 individuals that had just matured. In the catastrophe scenarios, we allowed the population to converge for 20 projection intervals and then eliminated all the juveniles or all the mature individuals. We used the standard parameters (Table 4.1).

Age distributions extremely different from the stable age distribution in the colonization scenarios proved to be a significant challenge to the simple two-stage matrix model. Two effects cause the large discrepancies in transients when newborns only are considered: the pooling effect and the numerical diffusion. The pooling effect, discussed in Section 4.4.1, results from the ability of the adults in the matrix model to pool their reproductive resources and produce newborns immediately, while in reality individuals must accumulate enough energy to reproduce. The numerical diffusion is a consequence of the inability of the matrix model to distinguish individuals within a stage, i.e. individuals 'diffuse' throughout the stage. Therefore, the matrix model overestimates maturation following colonization by newborn individuals because a certain proportion of individuals in the matrix model matures at each projection interval, while in reality individuals must stay in the immature stage until age $\tau_{p}$.

Both effects result in an overestimate of the number of newborns, and the matrix model predicts quicker-than-observed population recovery (Figure 4-11). Adding more stages to the matrix model would help ameliorate both effects. 

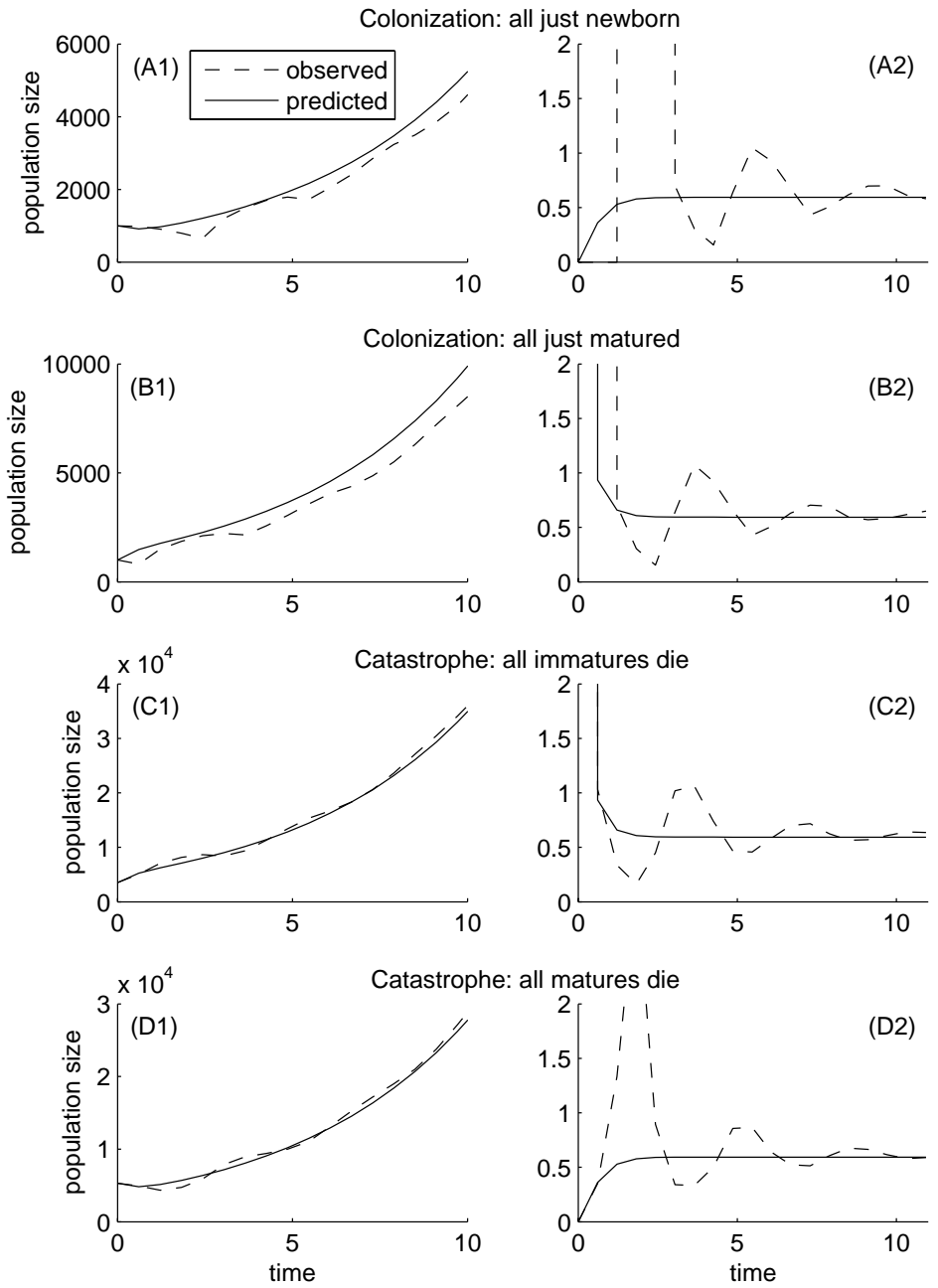

Figure 4-11: Colonizations: Plots (A): initial population is comprised of newly born individuals. Population size (A1) and ratio of matures and immatures (A2) shown. Plots (B): initial population is comprised of newly matured individuals. Population size (B1) and ratio of matures and immatures (B2) shown. Catastrophes: Plots $(C)$ : all immatures die. Population size $(\mathrm{C} 1)$ and ratio of matures and immatures (C2) shown. Plots (D): all matures die. Population size (D1) and ratio of matures and immatures (D2) shown. 


\subsection{Discussion}

The physiological responses of individuals to their environment determine their growth, survival and reproduction. In turn, these vital rates determine the dynamics of populations. In this paper, we have demonstrated how one can connect the physiological responses of individuals to population dynamics by constructing a matrix population model whose transition probabilities are determined by a DEB model. Although the analyses in this paper use a particular DEB model based on Kooijman's DEB theory, the developed framework (summarized in Appendix A) is general.

The construction process involved a number of approximations. To asses the impact of those approximations we compared the predictions of the matrix model to observations of an IBM whose individuals were governed by the DEB model. We found that, in general, predictions of the vital rates (fecundity, maturation probability, and survivorships) and of two asymptotic demographic statistics (population growth rate and stable stage distribution) closely matched the observations. Our approach tends to underestimate adult survivorship and overestimate fecundity. These mismatches are typically small, but are largest for rapidly declining populations. The mismatches can be attributed to two causes. First, it is difficult to estimate the vital rates from observations of the IBM when $\lambda$ is small because the number of stage transitions observed before extinction is limited. Second, the pooling effect (discussed in Section 4.4) becomes more important when $\lambda$ is small. This effect, present in other structured models (e.g. Nisbet et al., 1997), is caused by pooling the reproductive energy of organisms unable to reproduce individually into a common pool, which then provides enough energy to produce offspring.

In addition to comparisons of asymptotic statistics, we also compared transient population trajectories under a number of ecological scenarios. The match between the matrix-based predictions and the IBM-based observations were good when the initial age distribution within each stage was close to the stable distribution. However, when these distributions were not close, the matrix model poorly captured the 
transient dynamics, principally as a result of numerical diffusion (discussed in Section 4.4.3). The effects of numerical diffusion can typically be ameliorated by the inclusion of additional stages in the matrix model.

Many previous studies relate continuous time physiological models to demographic models. For example, de Roos et al. (1992) connect size-dependent growth, mortality and reproduction, obtained from a physiological model, to demographic dynamics using escalator boxcar train technique (see also de Roos, 1988; de Roos, 1997; de Roos et al., 1997). Ross et al. (1994) connect rudimentary zooplankton energy balance models to ecosystem dynamics using ordinary differential equations (see also Ross et al., 1993a, 1993b), while Nisbet et al. (1997) use the same type of equations to represent biomass dynamics of a Daphnia population starting from partial differential equations for individual growth based on simple physiological considerations. Dougherty et al. (2002) link a physiological model of bacterial growth to population dynamics through ordinary differential equations representing sugar availability, acid concentrations and energy storage. McCauley et al. (1996) investigate dynamics of a stage-structured demographic model formulated using delay-differential equations starting from physiology of a herbivorous zooplankton (see also Gurney et al., 1983; Nisbet and Gurney, 1983; Nisbet et al., 1989). Kooi and Kooijman (1999) investigate differences between discrete and continuous approaches to reproduction by incorporating a DEB model into partial differential equations describing two physiological stages of their demographic model, and then using a finite difference scheme to project the population through time (see also Murphy, 1983; Metz and Diekmann, 1986, Kooijman et al., 1989). In addition to connecting a physiological model to the McKendrick-von Forester population conservation equation (Kot, 2001, pp. 391-400), Ault et al. (1999) add a spatial model to investigate population dynamics of sea trout and pink shrimp. These studies highlight the fact that there are demographic models other than matrix population models that we could have used.

Choices among these demographic models depend both on biological considera- 
tions (the life cycle of the organism, the form of the data, and the biological question at hand) and on the tastes of the scientist. Matrix models have several strengths. For many organisms, classification by stage is more useful than classification by age, and stage-classified models are particularly easy to develop in matrix form. In addition, matrix models are currently used in the theory of life history evolution more than other stage-structured models (Roff, 1992; Stearns, 1992), as an approximation to McKendrick-von Forester population equations (Kooi et al., 2001), as well as a way to incorporate toxic effects observed in individuals into demographic models (Lopes et al., 2005). We hope to use our modelling framework to address evolutionary questions at another time.

Our analyses in this paper is based on a specific $\kappa$-rule DEB model. The approach we use requires only the solutions of our individual model: size, energy committed to reproduction, energy needed to reproduce, and the risk of death as functions of age. The same approach can be used with any other $\kappa$-rule DEB model (Kooijman 2001), as well as any other individual model that produces these outputs (e.g. von Bertalanffy, 1957; Kilgore \& Armitage, 1978; Wunder, 1978; Paloheimo et al., 1982; Huntley et al., 1987 (review); Hallam et al., 1990; Markussen et al., 1990; Persson et al., 1998; Hickie et al., 2000).

The principal advantage of a $\kappa$-rule model is that it is a supply side model, i.e. a model in which food availability determines the growth, survival and reproduction of individuals $^{2}$. As such, it enables us to analyze the interacting effects of physiology and environmental variability on population dynamics. We plan to investigate these interactions in the future.

The matrix model presented in this chapter has only one dimension: size, $V$. To capture growth at different food availabilities, the matrix model needs to account for energy density of individuals. This means that individuals have to be classified not

\footnotetext{
${ }^{2} \mathrm{~A}$ demand-side model, in contrast, determines what the energy intake must have been to have produced a prescribed pattern of growth and reproduction.
} 
according to size only, but energy density as well. I present a way to extend the matrix model to include energy as a state variable in the Appendix C.

\subsection{Acknowledgments}

We thank E. Montie for problems inspiring the project, and M. Hahn and S.A.L.M. Kooijman for insightful comments. The work of T. Klanjscek, H. Caswell and M. G. Neubert was supported by the David and Lucile Packard Foundation, the U. S. National Science Foundation (DEB-9973518, OCE-0083976), the U. S. Environmental Protection Agency (R-82908901) and the WHOI/MIT Joint Program in Biological Oceanography. R.M. Nisbet's work was supported by the STAR EaGLe program through the PEEIR Consortium (U.S. EPA R-882867601). WHOI contribution \#

\subsection{References}

Ault, J.S., Luo, J., Smith, S.G., Serafy, J.E., Wang, D.J., Humston, R. \& Diaz, G.A., 1999. A spatial dynamic multistock production model. Canadian Journal of Fisheries and Aquatic Sciences 56(suppl. 1):4-25.

Caswell, H., 1989. Analysis of life table response experiments .1. Decomposition of effects on population-growth rate. Ecological Modelling 46(3-4):221-237.

Caswell, H., 1996. Analysis of life table response experiments .2. Alternative parameterizations for size- and stage-structured models. Ecological Modelling 88(1$3): 73-82$.

Caswell, H., 2001. Matrix population models: construction, analysis, and interpretation. Sinauer Associates, Sunderland, Massachusetts, USA ISBN 0-87893-096-5.

Dougherty, D.P., Breidt, F.Jr., McFeeters, R.F. \& Lubkin, S.R., 2002. Energybased dynamic model for variable temperature batch fermentation by Lactoccus lactis. Applied Environmental Microbiology 2468-2478.

de Roos, A.M., 1988. Numerical methods for structured population models: The 
escalator boxcar train. Numerical Methods for Partial Differential Equations 4:173195.

de Roos, A.M., Diekmann O. \& Metz, J.A.J., 1992. Studying the dynamics of structured population models: A versatile technique and its application to Daphnia. American Naturalist 139:123-147.

de Roos, A.M., McCauley, E., Nisbet, R.M., Gurney, W.S.C \& Murdoch, W.W., 1997. What individual life histories can (and cannot) tell about population dynamics. Aquatic Ecology 31:37-45.

de Roos, A.M., 1997. A gentle introduction to models of physiologically structured populations. In: S. Tuljapurkar and H. Caswell (Editors), Structured-population models in marine, terrestrial, and freshwater systems. pp. 119-204, Chapman \& Hall, New York.

Fujiwara, M., Kendall, B.E. \& Nisbet, R.M., 2004. Growth autocorrelation and animal size variation. Ecology Letters 7:106-113.

Gurney, W.S.C., Nisbet, R.M. \& Lawton, J.H., 1983. The systematic formulation of tractable single-species models incorporating age structure. Journal of Animal Ecology 52:479-495.

Hallam, T.G., Lassiter, R.R., Li, J. \& Suarez, L.A., 1990. Modelling individuals employing an integrated energy response: application to Daphnia. Ecology 71(3):938954.

Hickie, B.E., Kingsley, M.C.S., Hodson, P.V., Muir, C.G., Beland, P. and Mackay, D., 2000. A modelling-based perspective on the past, present and future polychlorinated biphenyl contamination of the St. Lawrence beluga whale (Delphinapterus leucas) population. Canadian Journal of Fisheries and Aquatic Sciences 57:101-112.

Huntley, A.C., Costa, D.P., Worthy, G.A.J. \& Castellini, M.A. (Editors), 1987. Approaches to marine mammal energetics. Society for Marine Mammalogy, special publication No. 1. Allen Press, USA.

Kilgore, D.L., Jr. \& Armitage, K.B., 1978. Energetics of a yellow-bellied marmot 
population. Ecology 59:78-88.

Kooi, B.W. \& Kooijman, S.A.L.M., 1997. Discrete event versus continuous approach to reproduction in structured population dynamics. Theoretical Population Biology 56:91-105.

Kooi, B.W., Hallam, T.G., Kelpin, F.D.L., Krohn, C.M., Kooijman, S.A.L.M., 2001.

Iteroparous Reproduction Strategies and Population Dynamics. Bulletin of Mathematical Biology 63:769-794.

Kooijman, S.A.L.M., 2000. Dynamic energy and mass budgets in biological systems, 2nd edition. Cambridge university press, Cambridge, UK. ISBN 052178608 8.

Kooijman, S.A.L.M., Van Der Hoeven, N. \& Van Der Werf, D.C., 1989. Population consequences of a physiological model for individuals. Functional Ecology 3(3):325-336.

Kot, M., 2001. Elements of mathematical ecology, Cambridge University Press, Cambridge, UK ISBN 0521001501.

Lopes, C., Pery, A.R.R., Chaumot, A. \& Charles, S., 2005. Ecotoxicology and population dynamics: Using DEBtox models in a Leslie modeling approach. Ecological Modelling 188(1):30-40.

Markussen, N.H., Ryg, M. \& Oritsland, N.A., 1990. Energy requirements for maintenance and growth of captive harbour seals, Phoca Goenlandica. Canadian Journal of Zoology 68:423-426.

McCauley, E., Roger, R.M., de Roos, A.M., Murdoch, W.W. \& Gurney, W.S.C. 1996. Structured population models of herbivorous zooplankton. Ecological Monographs 66(4):479-501.

Metz, J.A.J. \& Diekmann, O., 1986. The dynamics of physiologically structured populations. Lecture notes in biomathematics, Vol. 68, Springer-Verlag, Berlin/NY.

Muller, E.B. \& Nisbet, R. M., 2000. Survival and production in variable resource 
environments. Bulletin of Mathematical Biology 62:1163-1189.

Murphy, L.F., 1983. A nonlinear growth mechanism in size structured population dynamics. Journal of Theoretical Biology 104:493-506.

Nisbet, R.M. \& Gurney, W.S.C., 1983. The systematic formulation of population models for insects with dynamically varying instar duration. Theoretical Population Biology 23:114-135.

Nisbet, R.M, Gurney, W.S.C., Murdoch, W.W. \& McCauley, E., 1989. Structured population models: a tool for linking effects at individual and population level. Biological Journal of the Linnean Society 37:79-99.

Nisbet, R.M., McCauley, E., Gurney, W.S.C., Murdoch, W.W. \& de Roos, A.M., 1997. Simple representations of biomass dynamics in structured populations. In: H.G. Othmer, F.R. Adler, M.A. Lewis, J.C. Dallon (Editors), Case studies in mathematical modeling - ecology, physiology, and cell biology, pp. 61-79. Prentice Hall, Upper Saddle River, New Jersey.

Nisbet, R.M., Muller, E.B., Lika K. \& Kooijman, S.A.L.M., 2000. From molecules to ecosystems through dynamic energy budget models. Journal of Animal Ecology 69:913-926.

Paloheimo, J.E., Crabtree, S.J. \& Taylor, W.D., 1982. Growth model of Daphnia. Canadian Journal of Fisheries and Aquatic Sciences 39:598-606.

Persson, L., Leonardsson, K., de Roos, A.M., Gyllenberg, M. \& Christensen, B., 1998. Ontogenetic scaling of foraging rates and the dynamics of a size-structured consumer-resource model. Theoretical Population Biolology 54(3):270-293.

Ren, J.S. \& Ross, A.H., 2001. A dynamic energy budget model of the Pacific oyster Crassostrea gigas. Ecol. Model. 142(1-2):105-120.

Ripley, B.J. \& Caswell, H., 2005. Recruitment variability and stochastic population growth of the soft-shell clam, Mya arenaria. Ecological Modelling 193:517-530.

Roff, D.A. 1992. The evolution of life histories, theory and analysis. Chapman and Hall, New York. 
Ross, A.H., Gurney, W.S.C. \& Heath, M.R., 1993a. Ecosystem models of Scottish sea lochs for assessing the impact of nutrient enrichment. ICES Journal of Marine Science, 50:359-367.

Ross, A.H., Gurney, W.S.C. \& Heath, M.R., 1994. A comparative study of the ecosystem dynamics of four fjords. Limnology and Oceanography, 39, 318-343.

Ross, A.H., Gurney, W.S.C., Heath, M.R., Hay, S.J. \& Henderson, E.W. 1993b. A strategic simulation model of a fjord ecosystem. Limnology and Oceanography, 38:128-153.

Stearns, S.C. 1992. The evolution of life histories. Oxford University Press, Oxford.

von Bertalanffy, L., 1957. Quantitative laws in metabolism and growth. Quarterly Review of Biology, 32(3):217-231.

Wunder, B.A., 1978. Implication of a conceptual model for the allocation of energy resources by small mammals. In O. Snyder (Editor), Populations of Small Mammals under Natural Conditions. Pymatuning Laboratory of Ecology, pp. 6875, University of Pittsburgh Special Publication Series 5, Pittsburgh, PA.

\subsection{Appendix A: Creating a matrix model based on a physiological model}

1. Pick a projection interval $\alpha$.

2. Obtain age-dependent size $(V(s))$, age-dependent commitment of reproductive energy $(\beta(s))$, and age-dependent probability of death conditioned on survival up to that age $(h(s))$ of an individual. For a DEB, use equations outlined in Table 4.5.

3. Estimate energetic cost $\left(C_{N}\right)$ of newborns.

4. Determine stages of the matrix model by dividing individuals into those younger than and older than the age at which individuals mature $\left(\tau_{p}\right)$. 
5. Assume a growth rate $\lambda_{1}$, e.g.

$$
\lambda_{1}^{\text {assumed }}=1
$$

6. Calculate the normalized stable age distribution using (4.15).

7. Calculate the transition probability $(\gamma)$ using (4.19) or (4.18) in case of more than two stages.

8. Calculate survivorships $\left(\sigma_{i}\right)$ for each stage using (4.21).

9. Calculate the average energy committed to reproduction per projection interval per mature individual from (4.22), the average number of newborns from (4.23), and the resulting fecundity $(F)$ using (4.25).

10. Assemble the projection matrix using (4.10).

11. Calculate the maximum eigenvalue of the projection matrix, $\lambda_{1}^{\text {calculated }}$.

12. Repeat steps 6-12 with

$$
\lambda_{1}^{\text {assumed }}=\lambda_{1}^{\text {calculated }}
$$

until satisfactory convergence of the growth rate is obtained. When $\lambda_{1}^{\text {calculated }}$ is very different from $\lambda_{1}^{\text {assumed }}$, the iterative process may be unstable because of possible overshoots that can lead to oscillations, rather than convergence. In such cases, in each iteration change $\lambda_{1}^{\text {assumed }}$ by only a fraction of the difference between $\lambda_{1}^{\text {assumed }}$ and $\lambda_{1}^{\text {calculated }}$ from the previous iteration.

\subsection{Appendix B - Calculating the stable age dis- tribution}

Let $N(s, t)$ be number of individuals of age $s$ at time $t$. Then the total number of individuals at time $t$ is

$$
N_{T}(t)=\int_{0}^{\infty} N(s, t) d s
$$


Table 4.5: DEB model equations

\begin{tabular}{lc}
\hline Description & Equation \# \\
\hline \hline Rate of change of energy density & $(4.1)$ \\
\hline Rate of change of volume of the structure & $(4.2)$ \\
\hline Flux of energy committed to reproduction & $(4.5)$ \\
\hline Number of newborns in a breading season (for 1 female) & $(4.26)$ \\
\hline Rate of change of the hazard rate & $(4.7)$ \\
\hline Rate of accumulation of damage-inducing compounds & $(4.8)$ \\
\hline
\end{tabular}

and the age distribution

$$
\phi(s)=\frac{N(s, t)}{N_{T}(t)} .
$$

From the definition of the hazard rate (4.6), we can calculate the probability that an individual survives to age $s$,

$$
\phi_{1}(s)=\exp \left(-\int_{0}^{s} h(x) d x\right) .
$$

The number of individuals of age $s$ at time $t$ is, then, the number of individuals born at time $t-s$ that survived until the time $t$,

$$
N(s, t)=N(0, t-s) \phi_{1}(s) .
$$

Furthermore, assuming the age distribution is constant and the intrinsic growth rate of the population $r$, the number of individuals of any age changes exponentially, so

$$
N(s, t)=e^{-r s} N(s, t+s) .
$$

Inserting (4.App.B.4) and (4.App.B.5) into (4.App.B.2) gives

$$
\phi(s)=\frac{e^{-r s} N(0, t) \phi_{1}(s)}{N_{T}(t)} .
$$

Since the age distribution is stable, the ratio of newborns to the total population is constant. Hence,

$$
\phi(s)=K e^{-r s} \phi_{1}(s) .
$$


Combining (4.App.B.3) and (4.14) with (4.App.B.7) and simplifying gives the stable age distribution (4.15).

\subsection{Appendix $\mathrm{C}$ - The extended matrix popula- tion model}

I follow the same general approach to the model as in the chapter above: first, I group individuals in stages, then I calculate the rates that govern transitions between these stages in a projection interval $\alpha$. Finally, I assemble the projection matrix from these rates. I group individuals according to their size $(V)$, and energy density $(e) ; V_{j}$ and $e_{i}$ denote the $j^{\text {th }}$ size and the $i^{\text {th }}$ energy stages, respectively. I estimate the transition probabilities between the stages from the simple DEB model (4.1-4.8) which describes the rates of change of energy density $(e)$, structural volume $(V)$, energy in the reproductive buffer $(\beta)$, and the hazard rate $(h)$ of an individual.

I define $P_{i j k l}$ as the probability that an individual of size $V_{l}$ and energy density $e_{k}$ at time $t$ grows to size $V_{j}$ and changes to energy density $e_{i}$ at time $t+\alpha$. For $j=1$, $P_{i j k l}$ represents fertility: the average number of offspring born with energy density $e_{i}$ by a female of size $V_{l}$ and energy density $e_{k}$.

I assume $P_{i j k l}$ has two parts. The first part, $R_{j l}\left(V_{l}, e_{k}\right)$, is the probability of growing from $V_{l}$ to $V_{j}$ during the projection interval. It depends on the energy density of the individual at time $t\left(e_{k}\right)$, but not on the energy density of the individual at time $t+\alpha\left(e_{i}\right)$. For $j=1, R_{j l}\left(e_{k}\right)$ represents the fertility of the individual of energy $e_{k}$ and size $V_{l}$.

The second part is the probability of changing the energy density from $e_{k}$ to $e_{i}$, $Q_{i k}\left(f, V_{l}, e_{k}\right)$. It depends on the food availability $(f)$ and the size of the individual at time $t\left(V_{l}\right)$, but does not depend on the final size of the individual $\left(V_{j}\right)$. The offspring are always born with the energy density equal to that of their mother.

These assumptions imply that changes in $V$ depend on the initial value of $e$, but are independent of changes in $e$. Similarly, changes in $e$ depend on the initial value 
of $V$, but are independent of changes in $V$. Then fertility and probability of growth are independent of probabilities of changes in energy density. Therefore, $P_{i j k l}$ is the product of $R_{j l}\left(V_{l}, e_{k}\right)$ and $Q_{i k}\left(f, V_{l}, e_{k}\right)$. Using a short-hand notation $R_{j l}^{(k)}$ and $Q_{i k}^{(l)}(f)$ for $R_{j l}\left(V_{l}, e_{k}\right)$ and $Q_{i k}\left(f, V_{l}, e_{k}\right)$, the transition probability $P_{i j k l}$ is then

$$
P_{i j k l}(f)=R_{j l}^{(k)} Q_{i k}^{(l)}(f)
$$

\subsubsection{Transitions between size classes, $R_{j l}^{(k)}$}

Here I calculate growth and reproduction assuming constant energy density between $t$ and $t+\alpha$. To calculate $R_{j l}^{(k)}$, the probability of transition from $V_{l}$ to $V_{j}$ for energy density $e_{k}$, I generalize methods presented earlier in this chapter. As in the simple matrix model, all size classes with energy density $e_{k}$ adhere to the stable age distribution $\phi^{(k)}(s)$ :

$$
\phi^{(k)}(s)=K\left(\lambda_{1}^{(k)}\right)^{-\frac{s}{\alpha}} \exp \left(-\int_{0}^{s} h^{(k)}(\xi) d \xi\right),
$$

where $s$ is the age of the individual, $\lambda_{1}^{(k)}$ the growth rate of the population when individuals have energy density $e_{k}$, and $h^{(k)}(s)$ the hazard rate of an individual of age $s$ and energy density $e_{k}$. Note that here we ignore past, present and future changes in energy density $e_{k}$.

Grouping individuals into stages implies that the individuals of the same size have the same age and transit from $V_{j-1}$ into $V_{j}$ at the same age, $\tau_{j}$. Hence, organisms in stage $j$ are of age $s \in\left[\tau_{j}, \tau_{j+1}\right)$. The ages of transitions between size classes $\left(\tau_{j}\right)$ depend on energy density and should be written as $\tau_{j}^{(k)}$. Since the dependence is clear from the context, I do not write the superscript to simplify the formulae.

First, I generalize $\gamma_{j l}^{(k)}$, the probability an individual of energy density $e_{k}$ grows from stage $l$ to stage $j$ conditioned on survival. It is equal to the proportion of 
individuals from stage $l$ that grew to stage $j$, but did not grow to stage $j+1$ :

$$
\gamma_{j l}^{(k)}=\frac{\int_{\min \left(\tau_{l+1}, \tau_{j}-\alpha\right)}^{\min \left(\tau_{l+1}, \tau_{j+1}-\alpha\right)} \phi^{(k)}(s) d s}{\int_{\tau_{l}}^{\tau_{l+1}} \phi^{(k)}(s) d s} .
$$

If an individual cannot grow through a stage in less than one projection interval, (4.App.C.3) simplifies to

$$
\gamma_{j l}^{(k)}=\delta_{j(l+1)} \frac{\int_{\tau_{j}-\alpha}^{\tau_{j}} \phi^{(k)}(s) d s}{\int_{\tau_{l}}^{\tau_{j}} \phi^{(k)}(s) d s},
$$

where $\delta_{x y}$ is the Kronecker $\delta$ symbol equal to one for $x=y$, zero otherwise.

Generalizing (4.21) gives the survivorship in stage $l$ :

$$
\sigma_{l}^{(k)}=\lambda_{1}^{(k)} \frac{\int_{\tau_{l}+\alpha}^{\tau_{l+1}+\alpha} \phi^{(k)}(s) d s}{\int_{\tau_{l}}^{\tau_{l+1}} \phi^{(k)}(s) d s}
$$

and generalizing (4.22) gives the average individual commitment of energy to reproduction:

$$
\overline{\Delta \beta_{l}}(k)=\alpha \frac{\left.\int_{\tau_{l}}^{\tau_{l+1}} \phi^{(k)}(\xi) \frac{d \beta^{(k)}(s)}{d s}\right|_{s=\xi} d \xi}{\int_{0}^{\infty} \phi^{(k)}(s) d s} .
$$

The flux of energy to reproduction, $d \beta^{(k)}(s) / d s$, can be calculated from equations (4.3)-(4.5) for $e=e_{k}$.

An individual committing $\overline{\Delta \beta}_{l}^{(k)}$ energy to reproduction produces a number of newborns equal to the ratio of the energy committed to reproduction and $C_{N}$, the cost of the newborn (see equation 4.23):

$$
N_{l}^{(k)}=\frac{\overline{\Delta \beta}_{l}^{(k)}}{C_{N}} .
$$

Finally, the fecundity of size class $V_{l}$ and energy density class $e_{i}$ is the number of newborns at time $t+\alpha$ per adult at time $t$ that survived to time $t+\alpha$ (see equation 4.25):

$$
F_{l}^{(k)}=\sigma_{l}^{(k)} N_{l}^{(k)}
$$


Then, $R_{j l}^{(k)}$ is

$$
R_{j l}^{(k)}=\left\{\begin{array}{cc}
\left(1-\Sigma_{j} \gamma_{j l}^{(k)}\right) \sigma_{l}^{(k)} & l=j \\
\gamma_{j l}^{(k)} \sigma_{l}^{(k)} & l<j \\
F_{l}^{(k)} \sigma_{l}^{(k)} & j=1 \\
0 & \text { otherwise }
\end{array}\right.
$$

\subsubsection{Transitions between energy classes, $Q_{i k}^{(l)}(f)$}

Here I calculate the changes in energy density assuming constant size between $t$ and $t+\alpha$. The transition between energy classes depends on $f$ and the volume of the individual at time $t\left(V_{l}\right)$. I calculate the transition probability assuming all individuals in size class $V_{l}$ have the same volume, $V_{l}^{*}$, which is the average volume in the size class weighted by $\phi^{k}(s)$, the stable age distribution for energy density $e_{k}$ :

$$
V_{l}^{*}=\frac{\int_{\tau_{l}}^{\tau_{l}+1} V(s) \phi^{(k)}(s) d s}{\int_{\tau_{l}}^{\tau_{l}+1} \phi^{(k)}(s) d s},
$$

where $\tau_{l}$ and $\tau_{l+1}$ are the ages of transitions between size classes that depend on $e_{k}$.

From 4.1, the rate of change of energy density of an individual of size $V_{l}^{*}$ is:

$$
\frac{d e}{d s}=v(f-e)\left(V_{l}^{*}\right)^{-\frac{1}{3}}
$$

Solving (4.App.C.11) gives the energy density of an individual of volume $V_{l}^{*}$ and energy intake $f$ at time $t+\alpha$ assuming the individual had energy density $e_{k}$ at time $t:$

$$
e^{(l) \prime}(f)=f-\left(f-e_{k}\right) \exp \left(-\frac{\alpha}{\tau_{e}^{(l)}\left(V_{l}^{*}\right)}\right)
$$

where

$$
\tau_{e}^{(l)}\left(V_{l}^{*}\right)=\frac{1}{v}\left(V_{l}^{*}\right)^{\frac{1}{3}}
$$

Let the new energy density $e^{(l) \prime}(f)$ of an individual of initial energy density $e_{k}$ fall between energy densities $e_{x}$ and $e_{x+1}$. Then, I assume that individuals from stage 
$e_{k}$ can only go to either stage $e_{x}$ or $e_{x+1}$, and that they distribute between these to stages so that the average energy equals $e^{(l) \prime}(f)$. Hence, if $q_{x}^{(l)}(f)$ is the fraction of individuals from $e_{k}$ that transit to $e_{x}$, the transition probability from $e_{k}$ to $e_{i}$ is

$$
Q_{i k}^{(l)}(f)=q_{x}^{(l)}(f) \delta_{i x}+\left(1-q_{x}^{(l)}(f)\right) \delta_{i(x+1)},
$$

where

$$
q_{x}^{(l)}(f)=\frac{e_{x+1}-e^{(l) \prime}(f)}{e_{x+1}-e_{x}} .
$$

Considering equations (4.App.C.12) and (4.App.C.13) suggests that larger individuals respond slower to changes in energy availability, and species with larger energy conductivity (larger $v$ ) respond faster.

\subsubsection{Calculating the projection matrix}

The transition probability from energy density $e_{k}$ and size $V_{l}$ to energy density $e_{i}$ and size $V_{j}$ is the product of the transition probability between size classes and the transition probability between energy classes (see (4.App.C.1)). It is, however, a tensor of the fourth rank, and cannot be used as the matrix population models which are of the form

$$
\mathbf{n}(t+1)=\mathbf{A n}(t)
$$

where $\mathbf{n}(t)$ is the population vector and $\mathbf{A}$ is the projection matrix with two dimensions. If there are $m$ size and $n$ energy density classes, the number of individuals of size $V_{l}$ and energy density $e_{k}$ is stored in the position $m(k-1)+l$ of the population vector.

Rearranging $P_{i j k l}$ in a similar fashion gives the projection matrix $\mathbf{A}$ :

$$
\mathbf{A}_{(m(i-1)+j)}(m(k-1)+l)(f)=P_{i j k l}(f) .
$$

The projection matrix has dimensions of $m n$, and the dominant eigenvalue of $\mathbf{A}$ gives the population growth rate. 


\subsubsection{Preliminary results}

I simulated environmental variability by varying energy availability on temporal scales longer (interannual) and shorter (seasonal) than one year. In both cases, I simulated a periodic environment, with periods of one projection interval for seasonal, and multiple projection intervals for interannual variability. The analysis of these two time scales requires different approaches because the matrix model is discrete in time, and cannot track changes on shorter time scales than the projection interval. For simulations, I used the extended matrix model with include five energy classes that span energy density from the minimum to the maximum simulated possible under the considered food availabilities.

\subsubsection{Seasonal variability}

To simulate seasonal variability, I represented the seasonal fluctuations of energy intake by a sinusoid with a period $\alpha$. I varied the characteristics of the fluctuations by varying the phase and the amplitude of the sinusoid (Figure 4-12):

$$
f(t)=f_{0}+f_{A} \sin \left(\frac{2 \pi}{\alpha} t\right)
$$

where $f_{A}$ is the amplitude of oscillations, $f_{0}=0.5$ is the average energy intake. Assuming the newborns are born with the average energy density $(e(0)=0.5)$, I calculated population growth rates (Figure 4-13) for the range of $f_{A}$ from the minimum (constant energy intake, $\left.f_{A}=0\right)$ to the maximum $\left(f_{A}=f_{0}\right)$ and phase from zero to $2 \pi$.

If the reproductive event happens when the food availability is increasing, the variability in food supply can be very large without significant consequences on the growth rate. Environmental variability reduces the growth rate, more so if the reproductive event happens when the food availability is increasing.

Next, I considered the impact of development time on the ability to buffer seasonal fluctuations. For a range of development times, I varied phase and amplitude of $f$ 
as shown in Figure 4-13 and found the minimum growth rate. I used the minimum growth rate as a measure of ability to buffer oscillations; the lower this minimum, the more poorly buffered the population is.

I changed the development time by increasing the size at which individuals mature $\left(V_{p}\right)$. This decreased the population growth rate. To make comparisons of growth rates reflect ability to buffer seasonal fluctuations rather than just the development time, I compensated the decrease caused by the prolonged maturation time by lowering the aging acceleration $\left(h_{a}\right)$ so that the maximum growth rates were equal for all development times.

The minimum growth rate increases with size (and time) to maturity (Figure 414), suggesting that species with longer time to maturity buffer seasonal variations better.

\section{Interannual variability}

I simulated interannual variability by changing the amplitude and period of the environmental fluctuations. The simulated environments had periods of $2 k$, where $k=0,1,2,3,4$ and 5 years, and an amplitude ranging from 0.1 to $f_{0}$ (Figure 4-15).

Simulations (Figure 4-16) suggest that organisms exploit interannual variability: the population growth rate increases with both the amplitude and period of interannual food intake variability. While I find it reasonable that the growth rate increases with the period because the longer times of plenty could compensate for the longer unfavorable times, I do not know why it should increase with variability as well. This is something I will investigate in the future.

\section{Discussion}

Individuals store and budget energy to protect themselves from short-, as well as longterm food variability. This protection may come at a cost in terms of reproduction when the food is abundant. Therefore, to maximize population growth (and, presumably, fitness), the individuals need to optimize the balance between committing 


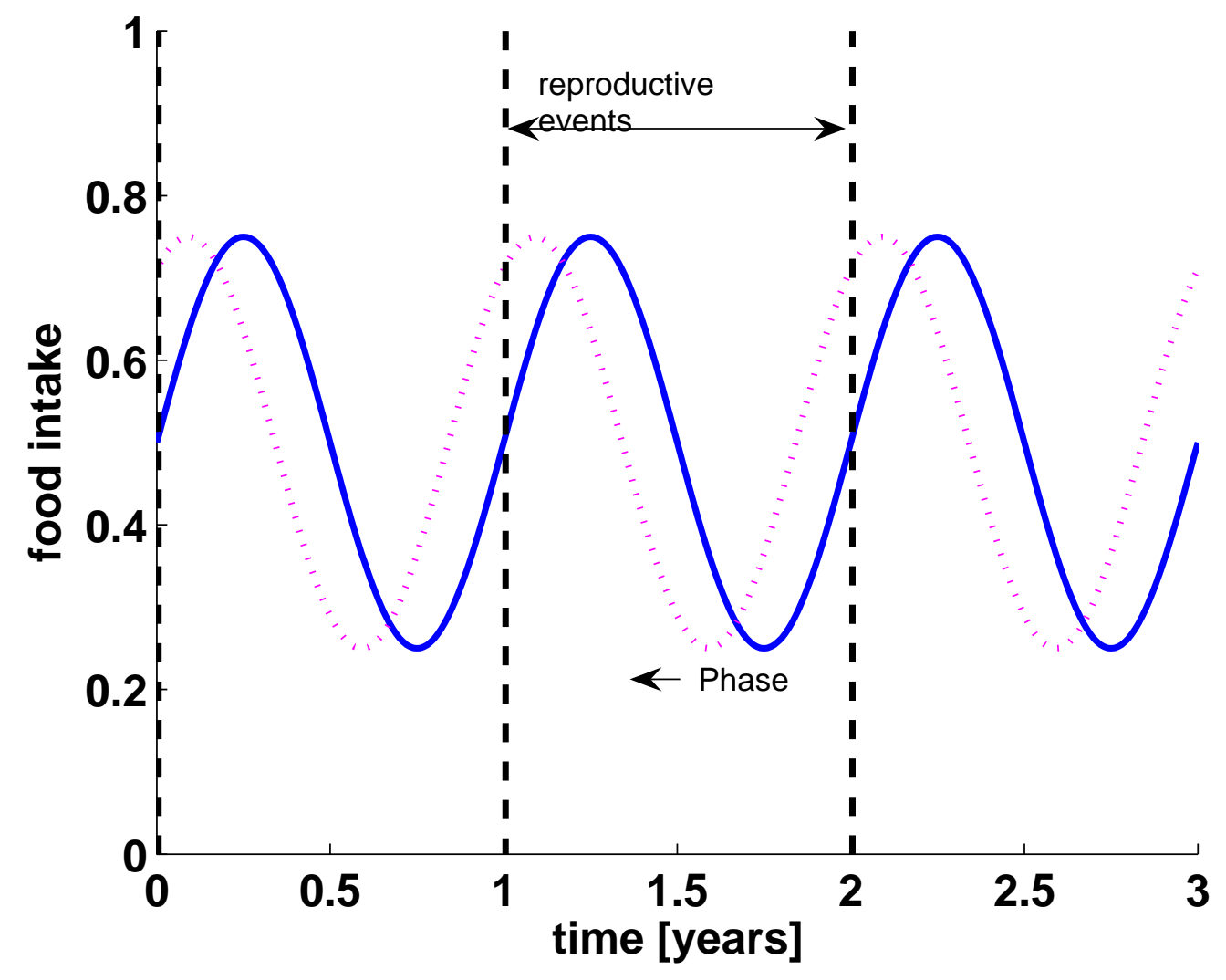

Figure 4-12: Two food intake scenarios. Both with amplitudes of oscillation 0.25, but with different phases: in phase with reproductive events (solid) and with a phase shift of approx. 60 degrees (dotted). 


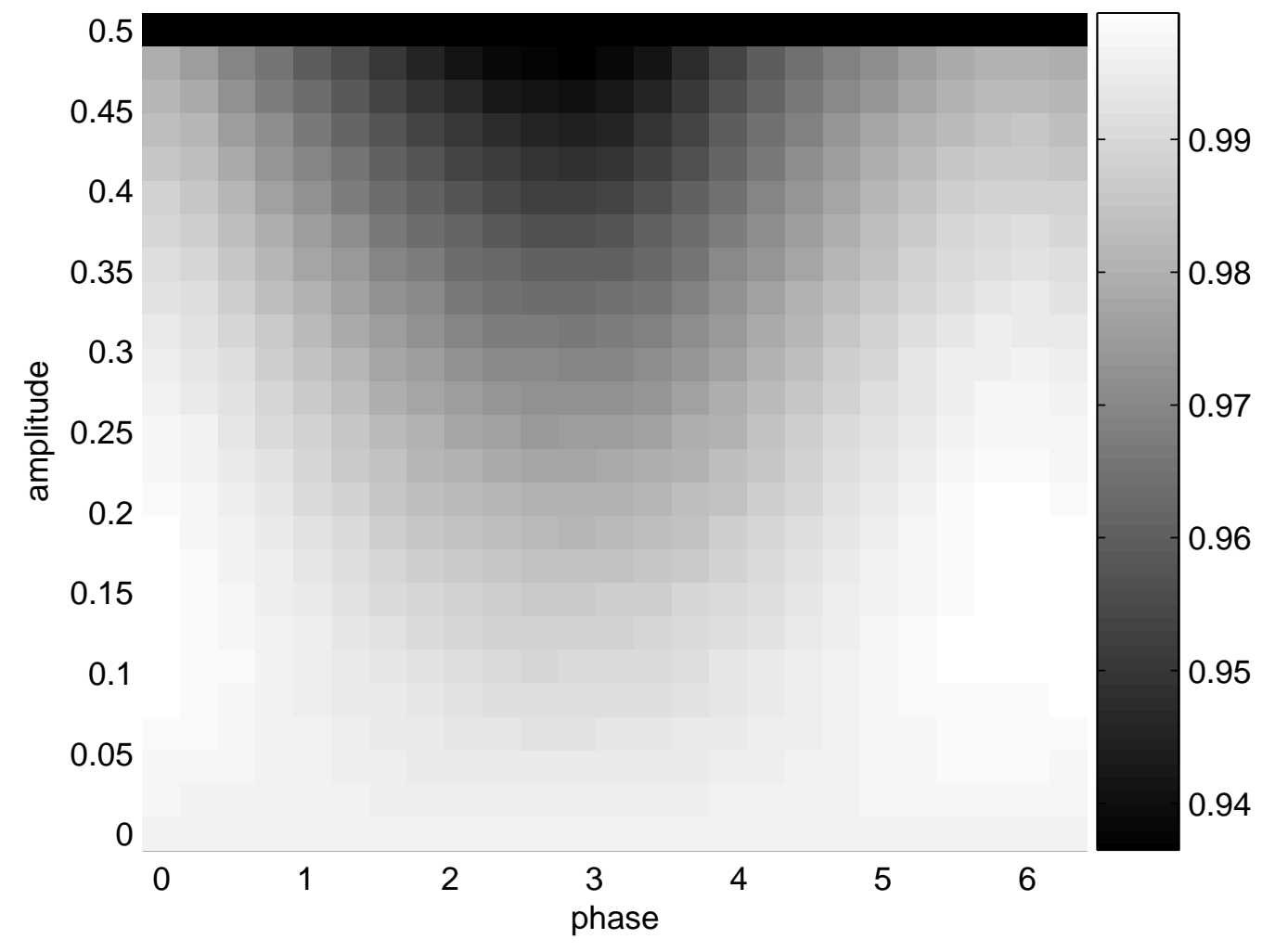

Figure 4-13: Growth rate in a seasonally varying environment. Zero amplitude corresponds to constant environment. 


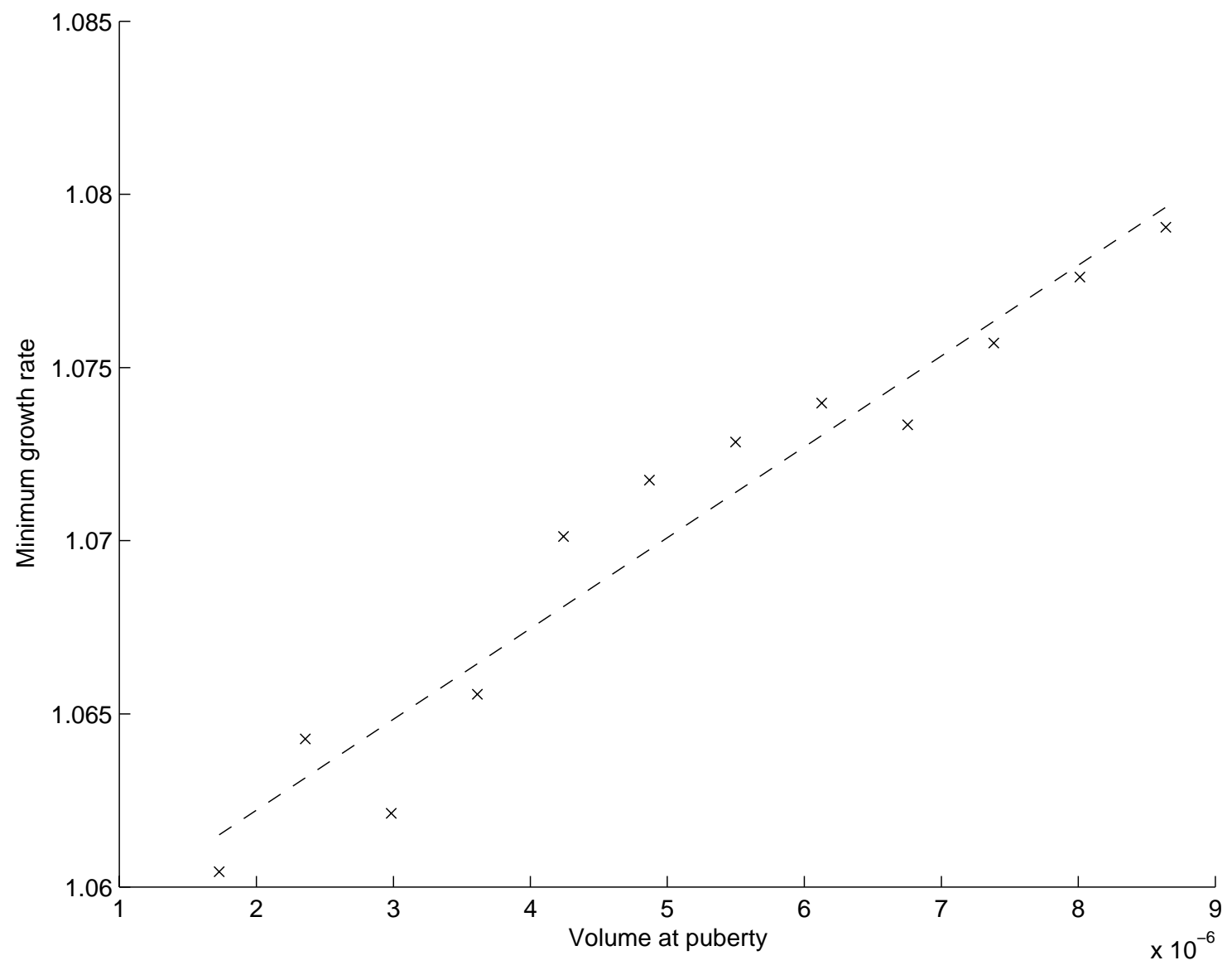

Figure 4-14: Minimum growth rate as development time increases. See text for discussion. 


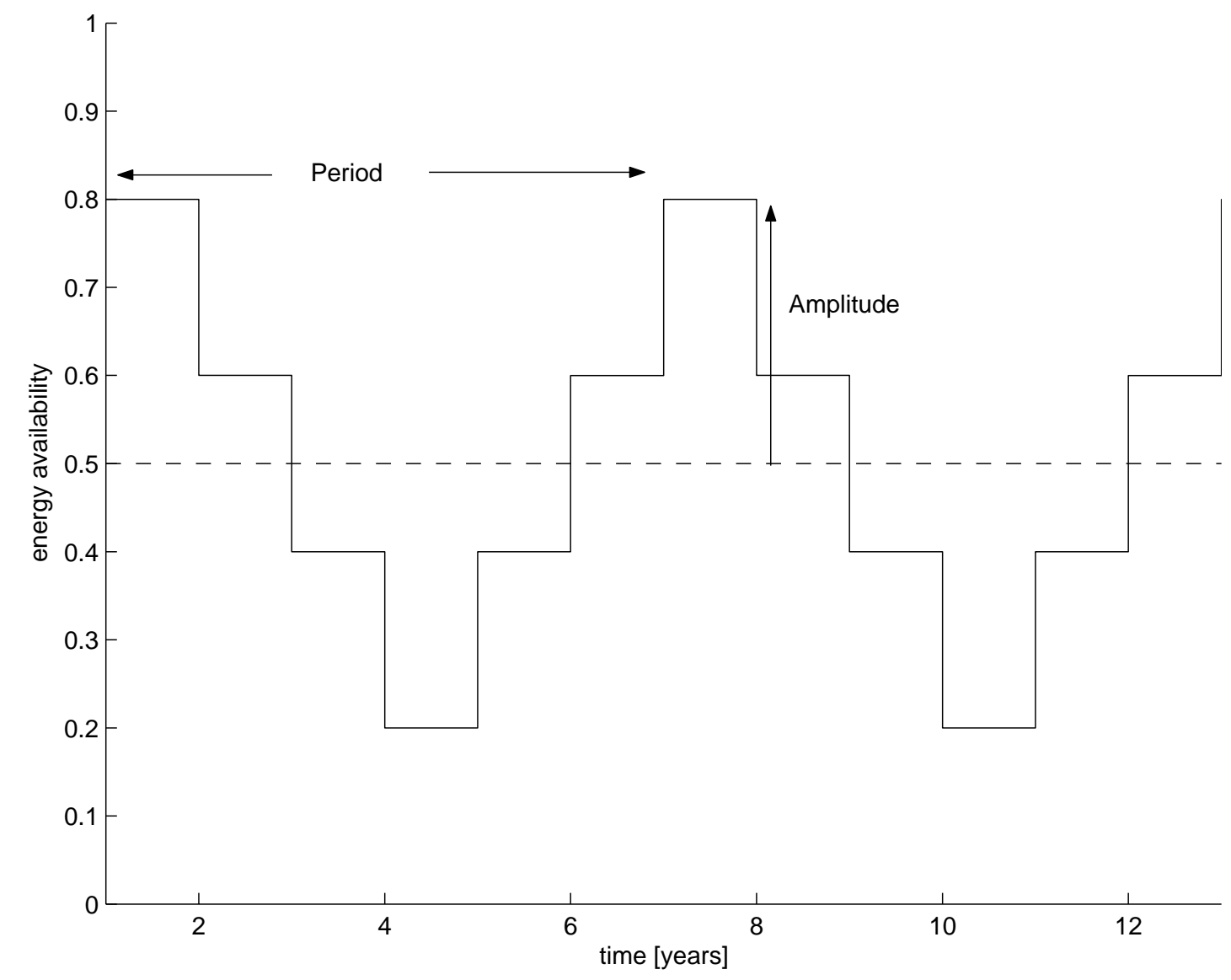

Figure 4-15: An example of a periodic environment. The period is 6 years $(k=3)$, and the amplitude is 0.3 . The dotted line represents the average energy availability $\left(f_{0}=0.5\right)$. 


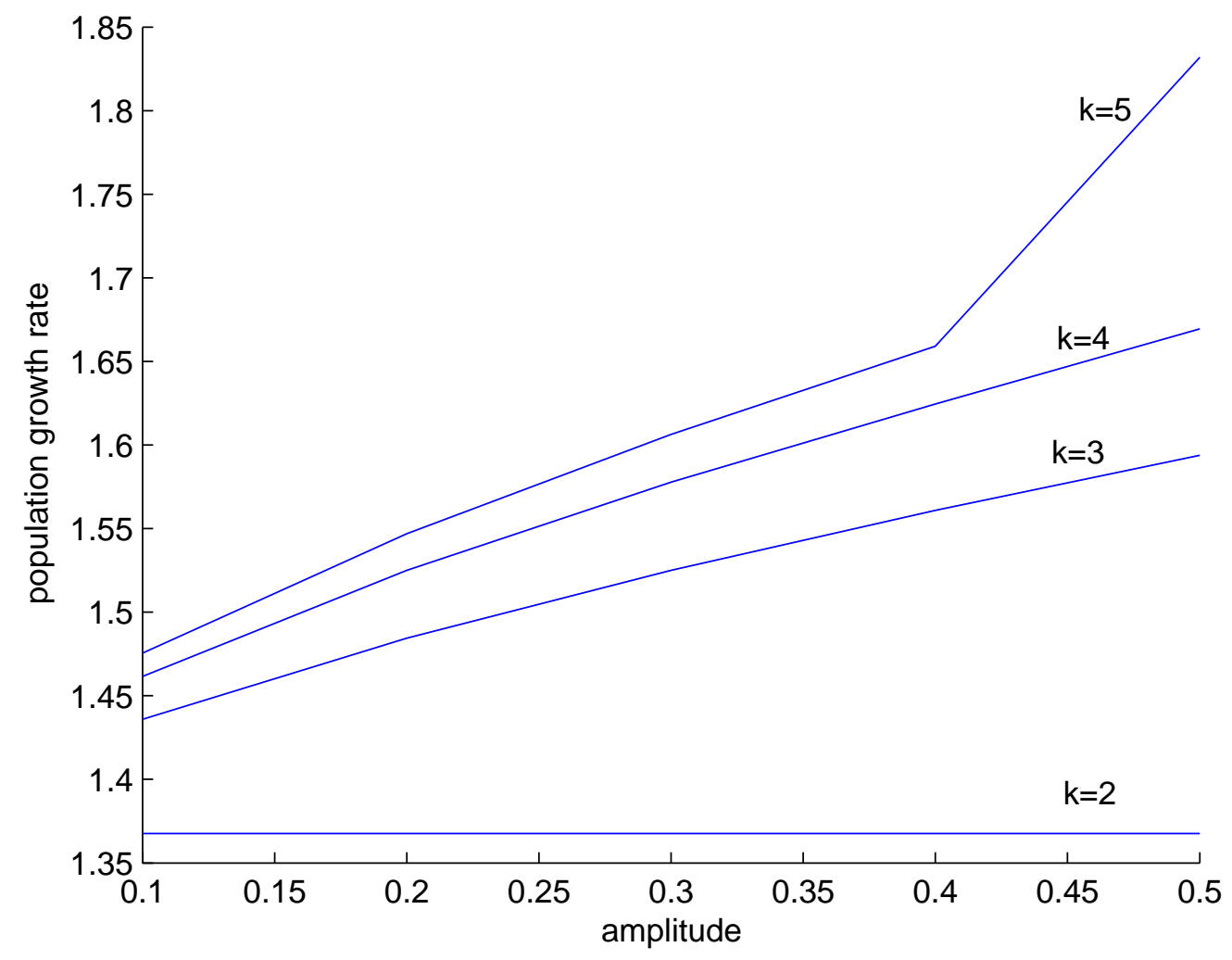

Figure 4-16: Growth rate as a function of period of interannual food intake variability $($ period $=2 k)$ and the amplitude of fluctuations. 
energy to reproduction, and storing the energy in the reserves to guard against environmental fluctuations. How well do energy budgeting scenarios cope with seasonal and interannual variability of energy intake?

For Mytilus edulis, the amplitude of oscillations in seasonal food availability and the timing of reproduction can interfere to reduce the population growth rate. If the organism reproduces just before the maximum food availability, the seasonal variability can be slightly beneficial compared to constant food availability. However, if the reproduction happens just before the minimum food availability, seasonal variability has negative consequences on the population growth rate. This is in stark contrast with the results for the right whale, where reproducing at the onset of minimum food availability minimizes calving interval (and maximizes reproduction).

Longer time to maturity (larger size required for reproduction) mitigated the negative effects of seasonal fluctuations. This suggests that organisms with short development times might be more susceptible to shifts in peaks of food abundance due to global climate change or human influence. 


\section{Chapter 5}

\section{Conclusion}

I may not have gone where I intended to go,

but I think I have ended up where I intended to be.

(Douglas Adams, writer)

This thesis introduces a number of theoretical advances, presents a tool to investigate right whale population dynamics, answers a number of questions, and raises new ones in the process. The theoretical advances and results are summarized in the outline of the thesis in Chapter 1, and covered in detail in Chapters 2-4. Instead of re-listing them here, I will comment on the bigger picture emerging from my thesis, reflect on some questions that come out of it, and suggest some possible future work and directions.

In this thesis I investigated how food availability, toxicants in the environment and maternal transfer of toxicants influence patterns of bioaccumulation of toxicants, growth and reproduction of individuals, and growth rate of populations. Figure 5-1 shows the general framework I used.

I chose and developed particular instances of each of the models within the framework. Using the dynamic energy budget (DEB) and pharmacokinetic models described in Chapter 2, I investigated how food availability and variability influenced individual growth, reproduction and patterns of bioaccumulation of toxicants. To 


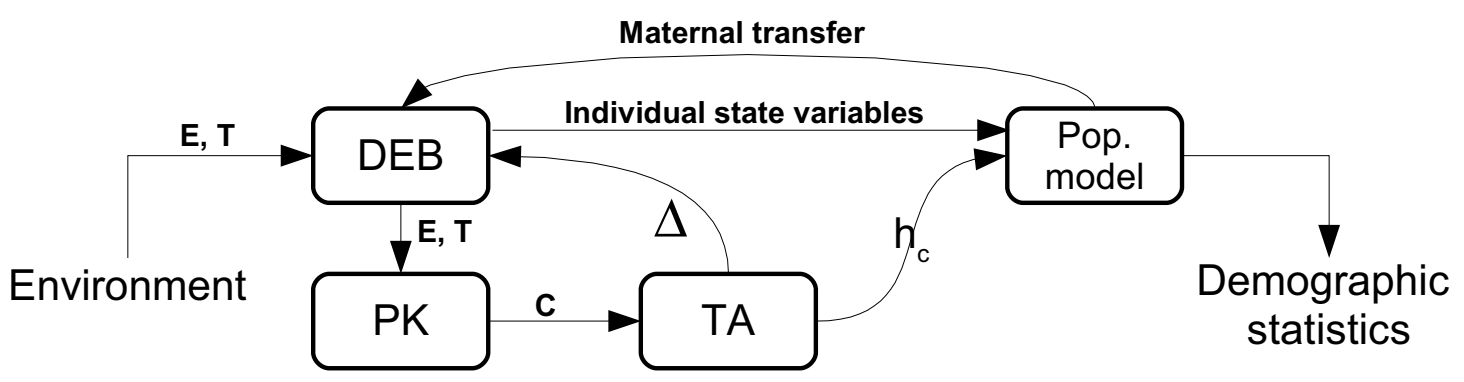

Figure 5-1: Modeling framework: energy $(\mathrm{E})$ and toxicants $(\mathrm{T})$ from the environment enter the dynamic energy budget (DEB) model, whose outputs are used by the pharmacokinetic (PK) model. The outputs from the PK model $(\mathrm{C})$ are interpreted by the toxicant action (TA) model as an increase in the hazard rate $\left(h_{c}\right)$ or a change in DEB parameters $(\Delta)$. The population model then uses the state variables of the DEB model and the TA model to calculate the demographic statisics such as population growth rate. Maternal exposure - the exposure of a marine mammal during fetal development and nursing - depends on another indivdiual (its mother) and requires a population model for proper implementation. 
investigate demographic statistics, however, I needed a population model. I took two approaches: individual based and a matrix population model.

In Chapter 3, I created and described in detail an individual based model (IBM) using the model of toxic action that determines the effects of toxicants on individuals (TA). I only considered direct effects of mortality ( $\Delta=0$ in Figure $5-1)$. Using the model, I was able to investigate population-level bioaccumulation patterns and effects of toxicants on marine mammals. All parts of the IBM are intimately connected and interact in complicated ways even though the equations governing them may be (fairly) simple.

I have taken great care to minimize complexity and only model crucial components of the biology. Nevertheless, the complexity of the IBM and the effort required to use it can be daunting. Furthermore, the many simplifications and assumptions I had to make to render the IBM manageable implies that one cannot expect the model to yield exact quantitative predictions. If the model is neither easy to use, nor yields reliable quantitative results, should it be used at all?

Certainly. Complexity of an approach should not be an obstacle to using it, if it answers questions that simpler approaches cannot. Ecological models rarely give exact quantitative predictions, but can be extremely elucidating in predicting patterns, discovering causalities and guiding further research. What questions should the individual model and the IBM (or parts thereof) be used for?

It could be used to create testable hypotheses. For example, is it really possible that a second-born calf receives more toxicants than the first-born calf? To answer this, we would preferably observe the mothers during multiple reproductive events. This is often not practical because of longevity of marine mammals, regulations and moral concerns. However, we can investigate the parameter space to find which species easier to experiment on should exhibit the same phenomenon. If the predictions of the hypothesis are confirmed by the experiment, we have a better understanding of processes of bioaccumulation. If the experiment disproves the hypothesis, some of our 
assumptions must have been wrong; finding which one improves our understanding of physiology.

The thesis offers more examples of questions (and answers) pertinent to the individual model and the IBM: from bioaccumulation patterns of individuals and populations, to maternal transfer and its effect on bioaccumulation patterns, to the effects of the environment on patterns of reproduction, growth and bioaccumulation. Many questions, like the consequences of feeding interruptions or climate change on right whales or how competition for resources with humans affects marine mammals, are yet to be answered.

Simulations of the IBM do not require pre-existing knowledge of growth, reproduction or bioaccumulation of individuals: the values of these variables are the result of simulations. If we were to use a simpler model, we would either have to incorporate the effects of not simulated parts of the framework shown in Figure 5-1, or ignore some of the biology. Incorporating the missing effects may require extensive knowledge of the quantities described by the missing parts of the framework, and the implementation may prove to be even more complicated than the IBM. Ignoring aspects of biology is not an ideal solution either, because we may be ignoring a vital part of biology without realizing it.

One of the most useful aspects of the IBM is its ability to point to parts of the model that do not significantly influence the outcome of interest and can be safely ignored. For example, we concluded that structural blubber does not play a significant reproductive and toxicological role in right whales if the percentage of the structural blubber is below $5 \%$. We also found that biotransformation of persistent toxicants can be neglected for most purposes. Both of these conclusions allow us to simplify our research by simplifying the IBM with the confidence that the results are still relevant to the organism.

The complete model provides a 'background' to test the less detailed, more manageable models against. For example, we tested three extreme scenarios of maternal 
transfer in Chapter 3. Results suggest that any mode of maternal transfer at most doubles the average concentration of toxicant in the blood. If we are interested in average concentration of a toxicant in the blood, and $50 \%$ is an acceptable error, we can safely ignore maternal transfer and possibly construct a very simple model we can use for subsequent analyses. The simple model may allow for more thorough analyses of the particular effect we are interested in, and provide us with a general insight practically unattainable with the full-blown IBM.

Therefore, the complete IBM provides a very powerful tool to investigate effects of the environment and human actions on individuals and populations. However, it should be used selectively and prudently because the inherent difficulties in coding and simulating such a complex IBM may prevent us from arriving at generalities otherwise attainable using simpler population models.

The simple and extended matrix population models presented in Chapter 4 are simpler than the IBM. The simple matrix model is a rudimentary two-stage matrix population model of Mytilus edulis in a constant food environment that ignores pharmacokinetics. I used such a simple model to develop and test the concepts required to connect the individual DEB to a more complex matrix population model. Since the simple model does not include variable energy levels or the pharmacokinetics, it is too simple to help investigate the interactions of food availability and exposure to toxicants on bioaccumulation and population growth rate.

The extended matrix model, presented in the Appendix $\mathrm{C}$ of the Chapter 4 is the next step towards a complete implementation of the framework in Figure 5-1 in matrix population form. In the extended model I account for energy reserves and arbitrary life cycles by expanding the simple matrix population model to include an arbitrary number of size (or physiology) stages, and explicitly include energy reserves. Changes in the toxicant concentrations could be added in a similar way using the assumption that transitions between toxicant concentrations depend only on the current size class, but not the transitions between size classes. The rules for transitions between 
toxicant concentrations would be different than those for transitions between energy densities; an algorithm similar to the one used to calculate transitions between size classes could be used.

Even without the addition of pharmacokinetics, the extended matrix population model offers a host of research directions. For example, using the population growth rate as a measure of the degree of success of a given energy budget in a given environment, one could compare energy budgeting strategies between species or search for the optimum energy budgeting strategy for a given relationship between reproductive intervals, expected life span and time scale of the environmental variability. Would the calculated optimum strategy for Mytilus correspond to the observed strategy? Why do whales have such different responses to environmental fluctuations than the Mytilus? How does the climate change factor into this?

Using the theoretical advances in this thesis, one could tackle a number of questions. How do patterns of bioaccumulation change if toxicants influence DEB parameters other than the hazard, as suggested by Muller and Nisbet (1997)? How do patterns of bioaccumulation change as the parameters of the DEB model change? How could the climate change affect the current outlook of endangered marine mammals? These and other questions can be investigated using the modeling framework in Figure 5-1.

The DEB and pharmacokinetic models presented in Chapter 2 are an integral part of the framework. Their exact forms may change somewhat depending on the question of interest, but their main structure will stay the same. The two demographic models, however, differ considerably and need to be chosen depending on the particular question. Each demographic model has its strengths and weaknesses.

The IBM is very flexible and is straightforward to run once implemented. It provides the greatest amount of detail, and information on all state variables of each individual. Distinguishing individuals allows direct observations of individual life histories which can then be used to calculate various quantities of interest, such 
as reproductive probability (e.g. Chapter 3). Its main disadvantages include the necessity to do simulations to arrive at vital rates and demographic statistics, and the long time it takes to do the simulations. The dependence of the results on the initial conditions complicates the matters further. The large amount of detail needed to run the model, and the huge amounts of data generated by every simulation can blur the big picture.

The matrix population model is a fast alternative: the initial simulations of the individual model are faster than a single simulation with the IBM, and the subsequent simulations are extremely fast, even not necessary for most purposes. Using general matrix population theory (Caswell 2001), one can calculate demographic statistics directly from the population projection matrix. Also, additional stressors can easily be added to the matrix model, thus facilitating the investigation of their effects on populations. Even though powerful and fast, matrix population models have disadvantages compared to the IBM: there is a considerable loss of detail, the numerous approximations needed to formulate the matrix model reduce its predictive ability, and grouping of individuals into classes precludes tracking of individual life histories. The method for estimating the transition probabilities is quite complicated, which reduces the usability of the matrix model.

In general, the IBM may be more appropriate when considering a particular species (a narrow range of parameters) and particular scenarios, or when detailed predictions are necessary. The matrix population model may be more suited for generalizations, when investigating a wide range of parameters, or when multiple simulations are necessary. Ultimately, the end does justify the means: the question at hand, available expertise and resources, as well as preferences of the scientist determine which model is more appropriate. 


\section{$5.1 \quad$ References}

Caswell, H. 2001. Matrix Population Models: construction, analysis and interpretation, second edition. Sinauer Associates, Inc. Sunderland, MA.

Muller, E. B., and R. M. Nisbet, 1997. Modeling the effect of toxicants on the parameters of dynamic energy budget models. in ASTM Special Technical Publication. ASTM, Conshohocken, PA, (USA). 